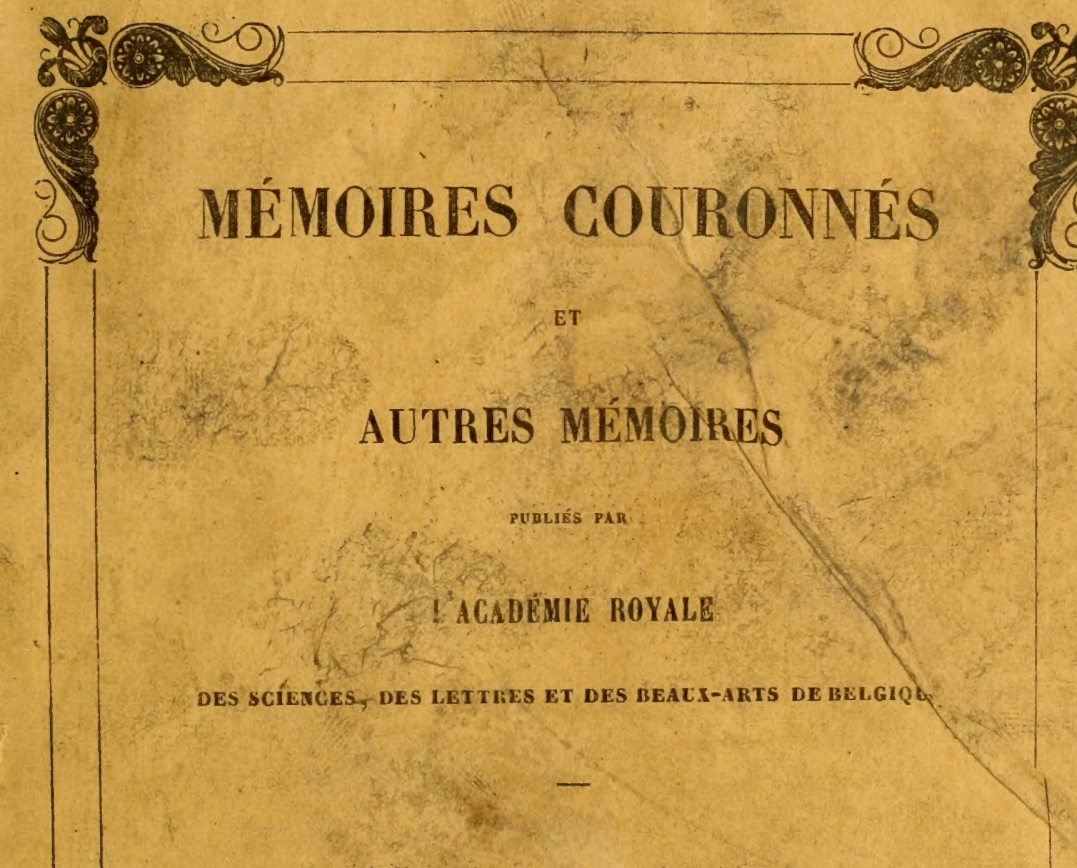

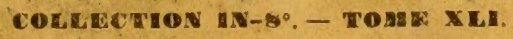

\title{
BRUXELLES,
}

F. HAYEZ, IUPLLUEUR DE L'ACADÉME RQYAIE DES SGIENCES, FPG., ET DE LACADÉME ROYAIE DE MEDEGINE DE BELGOQL,

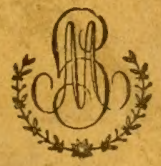

rue de Louvain, 108.

Octolire 1858 .

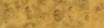


ma 


\section{MÉMOIRES COURONNÉS \\ ET}

AUTRES MÉMOIRES. 



\title{
MÉMOIRES COURONNÉS
}

ET

\section{AUTRES MÉMOIRES}

PUILLÉS PAR

\author{
L'ACADÉMIE ROYALB
}

DES SCIENCES, DES IETTRES ET DFS BEACX-ARTS DE BRIGIQUE.

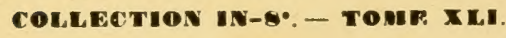

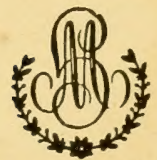

\section{BRUXELLES,}

F. HAYEZ, IMPRIMEUR DE L'ACADÉMIE ROYALE DES SGIENCES, ETC., ET DE L'ACADÉMIE ROYAISE DE MÉDECINE DE BELGIQUE,

rue de Louvain, 108.

Octobre 1888. 



\section{IIISTOIRE NATURELLE}

\section{KELL}

DES

\section{BALENOP'TERES,}

PAR

P.-J. VAN BENEDEN,

MEMBR: 2 DE L'ACADEMIE ROYALE DE BELGIQUE.

(Présenté à la Classe des sciences dans la séance du 10 mai 1887.)

TONE XLI.

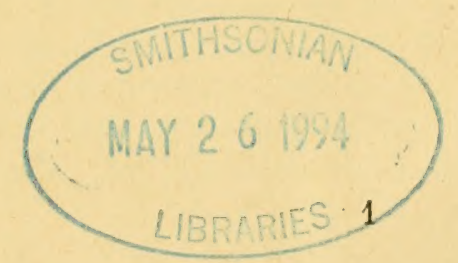





\section{HISTOIRE NATURELLE}

\section{DEs \\ BALENOPTERES.}

\section{LES BALÉNOPTĖRES.}

De tout temps, les baleiniers ont fait la distinction entre les Baleines, les Mégaptères et les Balénoptères, c'est-à-dire entre les Right wales, les Humpback et les Finback, qu'on appelle aussi Vinfisch.

Les naturalistes n'ont connu ces distinctions que fort longtemps après les baleiniers. Les récits de ceux qui avaient observé ces animaux étaient généralement incomplets, et les musées, même les plus importants, étaient dépourvus de restes de ces animaux.

Linné confondait, dans le genre Balcena, tous les grands Cétacés qui portent des fanons. Lacépède a proposé le nom de Balénoptère pour ceux qui ont une nageoire sur le dos.

Cuvier croyait à l'existence de deux espèces de Balénoptères : l'une, de la Méditerranée, représentée par le squelette de l'ani-

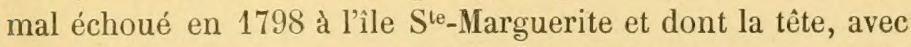
quelques os, est conservée au Muséum de Paris; l'autre, de la mer du Nord, d'après un animal jeté, en 1819, sur la côte du Holstein, et dont le squelette complet est conservé au Muséum de Berlin. Le troisième squelette, que Cuvier connaissait éga- 
lement, est celui qui est conservé à la maison de ville de Brême; et comme il provient d'un animal de petite taille, échoué à l'embouchure du Weser, Cuvier le croyait un jeune de l'espèce précédente.

Il y avait pour Cuvier un Rorqual de la Méditerranée et un Rorqual du Nord. Le grand naturaliste avait préféré le mot Rorqual, donné par les Norvégiens à des Baleines qui portent des tuyaux sous la gorge; il croyait à l'existence d'une Jubarte, espèce supposée sans plis sous la gorge.

Cuvier n'avait pas assez de matériaux à sa disposition quand il a écrit ses Recherches sur les ossements fossiles des Cétacés, et ce n'est que quelques années plus tard, que feu mon ami Eschricht a commencé ses intéressantes publications sur les Cétacés. Grâce surtout aux précieux et nombreux squelettes et foetus que son ami Hollböll lui envoyait du Groënland, le savant naturaliste de Copenhague a fait connaître les principales espèces de Balénides, en même temps que les caractères sur lesquels elles reposent.

Eschricht a fait faire un pas immense à la Cétologie, en démontrant que le nombre de vertèbres est le même dans le jeune âge qu'à l'âge adulte, que celles-ci ne se soudent pas à un àge avancé, après avoir été séparées d'abord, et que tous les caractères de l'adulte se trouvent déjà dans le fœetus.

En même temps le savant naturaliste de Copenhague a fait voir, ce que l'on semblait également ignorer, qu'il existe une Balénoptère de petite taille, ne dépassant pas 30 pieds de longueur, qui n'a pas plus de quarante-huit vertèbres, qui a des fanons jaunes et des nageoires pectorales à moitié blanches, et que c'est elle qu'0thon Fabricius a eu l'occasion d'observer en vie pendant son séjour au Groënland.

Depuis les travaux de Cuvier, il a été reconnu également que la Balénoptère de la Méditerranée est l'espèce commune de l'Atlantique, et, dès 1836, nous avions signalé sa présence sur la côte d'Islande, d'après des caisses tympaniques que Gaimard avait rapportées de son voyage au Nord.

Nous voilà donc en présence de deux espèces bien distinctes, 
la Balcenoptera musculus et la Balanoptera rostrata, auxquelles vient s'en joindre une troisième de taille moyenne que Cuvier avait cru être la seule espèce du Nord : c'est la Balcenoptera borealis.

Les deux premières pénètrent de temps en temps dans la Méditerranée, surtout la seconde. Comme nous le verrons plus loin, ces balénoptères se distinguent parfaitement les unes des autres par leur's caractères extérieurs, aussi bien que par leur genre de vie et leur taille.

On connaît aujourd'hui une quatrième espèce, la plus grande de toutes, que Pierre Camper avait déjà mentionnée sous le nom de Strypireydr, et qui fréquente les courants glaciaires à côté de la Baleine franche; c'est l'espèce qui atteint la plus forte taille puisqu'elle a jusqu'à 80 pieds de longueur; Gray a proposó de la nommer Balanoptera Sibbaldii.

Après Eschricht, c'est à M. Flower que nous devons les principaux progrès accomplis dans cette étude. Le savant directeur du British IIuseum s'est occupé particulièrement des individus échoués sur les côtes d'Angleterre, et il a largement contribué à faire disparaître les nombreuses erreurs qui araient été introduites dans la Cétologie.

M. Flower a fait connaitre aussi divers faits anatomiques intéressants, parmi lesquels nous derons citer la composition du bassin de ces animaux et les caractères propres aux os nasaux.

Schlegel ne croyait pas deroir admettre plus d'une espèce dans les mers septentrionales.

On trouvera plus loin le nom de ceux qui ont également contribué à mieux faire connaître ces Cétacés.

Le genre Balanoptera peut se caractériser par la nageoire que l'animal porte sur le dos, par les membres pectoraux qui sont petits, par les fanons qui sont courts et par des tuyaux ou plis que l'animal porte sous la gorge et qui s'étendent jusqu'à l'abdo- 
men. Il se distingue en même temps par la tête, qui a le quart de la longueur du corps, par le rostre qui est très peu courbé, comme par les vertèbres cervicales qui sont toutes séparées.

Comme caractère distinctif des espèces, nous croyons pouvoir accorder une grande valeur aux fanons: la Balcnoptera Sibbaldii a les fanons d'un noir bleuâtre uniforme; la Balcenoptera musculus a les fanons verdâtres ou pâles, avec des lignes pâles dans toute la longueur; la Balcenopteru borealis a les fanons noirâtres avec les barbes blanches et soyeuses; la Balcenoptera rostrata a les fanons d'un jaune pâle.

La femelle des Balénoptères est en général plus grande que le mâle, comme dans les autres Cétacés à fanons.

L'accouplement, comme la parturition, a lieu en hiver; la gestation paraît être de dix d̀ douze mois. En venant au monde, le jeune a le quart de la longueur de la mère 1.

Le jeune accompagne sa mère jusqu'à ce qu'il ait la moitié de sa taille.

La Balanoptera Sibbaldii parait faire exception pour la durée de la gestation aussi bien que pour l'époque de l'accouplement et de la parturition.

D'après une observation faite au détroit de la Sonde, par le professeur Giglioli, les Cétacés qui nous occupent seraient également sujets à l'albinisme.

Le système nerveux, à l'åge embryonnaire, a été l'objet de recherches importantes au haboratoire de zoologie de l'Université de Liège, par le $\mathrm{D}^{\mathrm{r}}$ Guldberg ${ }^{2}$ de Christiana, et le cerveau de l'adulte a été étudié avec soin par M. Beauregard 3. Cette

${ }^{1}$ Les vraies Baleines, comme les Célodontes, ont le tiers de la longueur de la mère en naissant.

${ }^{2}$ Gulderig, Ueber das Centralnervensystem der Bartenwale. Christiania, 1885. (Christiania videnskab Forhandlinger, 1885, $\mathbf{1}^{\circ} 4$. ) -- Guldberg, Ueber die Grössen- und Gewichtsverhälnisse des Gehirns bei den Bartenwalen.. Meddelelser fra den Naturhistorishe Forening i Kristiania, 188 s̀.

5 Journal d'analomie et de physiologie, t. XIX, 1883. 
étude a conduit l'aide naturaliste du Muséum de Paris au même résultat auquel Broca était arrivé, c'est-ì-dire, que les Cétacés, par la conformation du cerveau, se ramènent à un type peu différent de celui des Solipèdes et des grands Pachydermes.

Les Balćnoptères, comme les autres Cétacés à fanons, se distinguent par le développement de leur lobe olfactif des Cétacés à dents.

Le larynx des Balénoptères présente également un caractère qui ne se: trouve pas chez le's Cétodontes, mais qui leur est commun avec tous les Cétacés à finons: il est pourvu d'une poche, sac laryngé, qui peut au besoin se remplir d'eau et dont le calibre est assez grand pour avoir été confondu avec l'intestin. Ce sac laryngé semble représenter les poches des évents des Cétodontes et l'eau qu'il renferme peut se mêler à l'air au sortir des évents.

L'intestin des Balénoptères se distingue aussi jar un coecum qui n'existe pas dans les vraies Baleines, et, dans ces derniers temps, MIM. Beauregard et Boulard ont fait connaître les particularités de leur appareil génito-urinaire 1.

Chez certaines Balénopteres il existe une tendance particulière à la bifidité de la première côte. C'est à tort que des zoologistes avaient eru devoir accorder une certaine importance à cette disposition qui est purement individuelle.

Les pêcheries de la côte de Fimmark ont fait comnaître en partie les mours de ces animaux.

Le régime n'est pas le même dans les différentes espèces de Balénoptères; les unes poursuivent surtout les banes de Mallotus, de Harengs ou de Gades: la Balinoptera musculus et la Balcenopter rostrata ; les autres se repaissent de Crustacés assez. petits; leur estomac en est toujours plein sur la côte de Finmark : dans la Baldenoptera Sibbaldii, on trouve l'Euphliasia

${ }^{2}$ Journal d'analomie et de physiologie, 1862. 
inemis Thysanopode), dans l'autre, la Balanoptera borealis, le Calanus finmarchicus.

Les baleiniers s’accordent ì dire que la voracité des Balénoptères est si grande que, quand elles se trouvent au milieu d'un bane de Mallotus, elles ne s'occupent pas plus d'un steamer qui approche que du rent qui ride la surface de la mer.

Il est à remarquer que ces dernières espèces de Balénoptères ont des tanons noirs: la Bulenoptera borealis avec les barbes toutes fines et blanches, la Balienoptera Sibbaldii arec les mêmes barbes grosses et noires.

Chaque espice souffle et lespire à sa manière, et les baleinier's distingutent fort bien à distance les espèces qui applaraissent sur l'horizon. En général, on peut dire qu'elles se tiemnent dans une position horizontale en renant à la surface, qu'elles respirent trois ou quatre fois, puis plongent en levant la queue hors de l'ean. Le's baleiniers disent que c'est la Bulunuptera Sibbuldii qui relève le plus la queue, la Bulcenoptera musculus, le moins.

Sur le's côtes de Finmark on voit ordinairement la Balkenoptera Sibbuldii dominer; mais en $1883 \%$, cétait la Balcenoptera borealis. Cette dernière ne compte dans les années ordinaires que pour un sixième.

Les Balénoptères émigrent toutes périodiquement.

Les Balénoptères, comme les Harengs, et sans doute comme bien d’autres animaux marins, émigrent à la même époque de l'année, mais ne pénètrent dans certaines régions que pour autant qu'elles y trouvent certaine pâture dont la présence dépend de la température de l'eau. On a fait depuis longtemps l'observation, que les Baleines franches descendent plus bas sur la côte de Labrador que sur la côte du Groënland; les glaces sont plus abondantes du cîté de l'Amérique que du côté opposé.

On a observé que les Balénoptères atteignent une latitude plus élevée dans l'Océan glacial, en automne, quand les eaux sont chauffées pendant les mois d'été, qu'au printemps. En mai on ne les roit pas au delà de $70^{\circ}, 40 \%$, tandis qu'en septembre elles remontent jusqu'à $78^{\circ}$ (Leslie). 
M. Alfred Cocks a rencontré des Balénoptères en automne 1882, au $1^{\text {er }}$ septembre, tout près des glaces (la température de l'eau était de $1.3^{\circ}$ au-dessus de zéro. Elles n'étaient que trois ou quatre; le.3 stptembre suivant, il en aperçut encore deux à

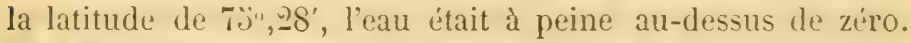
M. Alfred Cucks ne sait dire le nom de lespèce qu'il a ell suus les yeux; cela peut être la Bulenoptera Sibbaldii et les moins grandes des Megaptera, dit-il.

Nous arons publié une Notice sur la distribution géographique des Balénoptères, dans les Bulletins de l'Académie 1, en tenant conspte surtout des échouements, qui ont eu lieu depuis les côtes de Norrège jusqulaux cûtes de Portugal, comme à l'intérieur de la Méditerranée et de la Baltique. Mais ces échouements ne nous ont rien appris; en général, comme l'a dit Eschricht, ces animaux n'échouent que sur les côtes qüils ne visitent guère.

On roit apparaître les Balénoptères tous les ans rers le mois de mai dans l'Atlantique; elles se rendent, les unes vers la mer de Baflin, les autres vers l'Islande et la mer de Barentz, où elle's restent pendant les mois d'été; au mois daoùt elles abandonnent ces parages pour se rendre dans des eaux plus méridionales.

On a remarqué que la Bulenopteru musculus arrive la première sur les cótes de Finmark et la Balcenoptera Sibbaldii la dernière; la $B$. musculus se montre parfois déjì à Ia fin de l'hiver. Sophus Hallas a indiqué, dans une intéressante notice, les observations qu'il a eu l'occasion de faire sur les Cétacés des eaux d'Islande. Il a vu la Bulcenopteru Sibhaldii, que les Islandais appellent Strypireyslr, depuis le 16 juin jusqua $10 \ddot{\text { septrmbre, }}$ et une autre espèce dont il ne dit pas le nom et qui est probablement la Balconoptera musculus.

2 ge série, 1 XLV, mars, 1878 . 
On a longtemps pêché les Baleines sans songer à faire la chasse aux Balénoptères; on ne poursuit guère les Balénoptères, observait Holböll, gouverneur du Groënland; leurs fanons sont sans usage, l'animal donne peu d'huile et leur pêche est difficile.

Les vraies Baleines ayant considérablement diminué partout, on a songé à mettre à profit les steamers et la poudre pour faire la chasse aux Balénoptères, et, en 186\%, une Compagnie anglo-anéricaine a commencé la pêche de ces Cétacés dans les eaux de l'Islande. Cette Compagnie a poursuivi cette industrie pendant $186 \Xi$, 1866 et 1867 .

Le capitaine Bottemanne, qui dirigeait cette pèche, m’écrivait, à la date du 17 juillet 1868, qu'il se trouvait quatre espèces de Balénoptères dans les eaux d'Islande, dont une lui était moins bien conmue que les autres, qu'il avait préparé le squelette de celle qui est connue sous le nom de Steypireydr.

Vers cette époque, un pêcheur norwégien, M. Sven Foyen, après avoir fait la chasse aux phoques dans les eaux de Jan Meyen, où il avait capturé jusqu' 22,000 individus en une saison de deux mois, a commencé la chasse aux Balénoptères sur les côtes de Fimmark, et, grâce aux perfectionnements qu'il a apportés successivement aux projectiles, il existe anjourd'hui des établissements sur toute l'étendue de la côte de Finmark.

Les steamers sortent le matin, soit de Vadsö, soit d'un autre port des environs, et ils reviennent le même jour; rarement ils quittent les lieux de pêche sans avoir capturé une Balénoptère. Ils remorquent le corps jusqu'au chantier, où l'on enlève ses fanons, sépare la graisse, et transforme ce qui reste en guano de Baleine.

Au début de cette pêche on ne chassait que la grande espèce, la Balenoptera Sibbaldii; aujourd'hui on ne néglige même pas la Balanoptera musculus, qui donne le moins de profit.

Les premières années qu'on se livrait à cette chasse dans le Varanger-Fiord, ces animaux y étaient si abondants pendant les mois d'été, que la surface de la mer paraissait par moments 
en ébullition; les navires osaient à peine se hasarder au milieu de cette surface vivante, qui s'étendait à perte de vue. On y prenait régulièrement des individus qui n’avaient pas moins de quatre-vingts pieds de longueur. En même temps, ces Cétacés se laissaient tous approcher sans fuir, comme partout ailleurs où les animaux se trouvent pour la première fois en face de l'homme.

Aujourdhui ces Cétacés ne pénètrent plus guère dans ce Fiord, et ceux que l'on chasse à l'entrée n’atteignent plus guère toute leur taille; ils sont devenus très farouches; on ne les approche que bien diflicilement, mais les engins sont plus perfectionnés et les steamers mieux appropriés a leur destination.

Pendant l'été de 1871, le capitaine Sven Foyen a capturé 38 Balénoptères, en $1873 \%$, 45 , en 1879 , ce nombre s'élève à 81 , en 1881 , il atteint 104 .

M. Alfred Cocks rapporte que, sur 406 Balénoptères capturées en 1883 sur les côtes de Finmark, il y avait $173 \mathrm{~B}$. Sibuculdii et le restant moitié $B$. musculus et $B$. borealis.

Indépendamment de ces trois espèces, on voit de temps à autre dans ces mìmes parages la petite espèce, la Bulcunopter rostrata et la Megaptera boops.

On a remarqué, avons-nous dit plus haut, que c'est la grande espèce qui remonte le plus haut, puisqu'on la trouve jusqu'au milieu des glaces à côté de la Baleine franche.

Ces différentes espèces sont-elles confinées dans ces parages, comme le sont les rraies Baleines, où les voit-on encore dans d'autres mers?

Nous venons de voir que, dans notre hémisphère, la grande Balénoptère n’a guère élé observée que dans les parties les plus septentrionales de l'Atlantique. Mais est-elle confiné dans ces régions?

Contrairement aux Baleines véritables, les Balénoptères sont probablement toutes cosmopolites, et on trouve les quatre formes de nos régions septentrionales, aussi bien dans l'Atlan- 
tique méridionale, que dans l'océan Pacifique, la mer des Indes et l'océan Austral.

On voit des Finbacks dans toutes les mers, dit le capitaine Jonan 1. Nous en avons rencontré, dit-il, dans l'Atlantique nord et sud, dans la Méditerranée, en grande quantité aux environs des Iles Malouines, à la côte du Chili, au Cap de Bonne-Espérance, dans les eaux de Madagascar, dans la mer d'Oman, dans le golfe de Bengale, au Japon, etc. Le capitaine d'Crville et, plus récemment, le professeur Hoseley, en signalent un grand nombre dans les mers du pôle austral.

Partout on parle de quatre espèces différentes par la taille aussi bien que par les caractères extérieurs.

Depuis longtemps nous avons été frappé de voir une petite Balénoptère, décrite et figurée par le capitaine Scammon, hanter l'océan Pacifique, ayant tous les caractères de notre Bulcenopter rostrutu. La taille, la couleur et tout le squelette sont si semblables, que nous n'arons pu nous empêcher de dire que notre petite Balénoptère se trouve également dans la mer Pacifique. Nous avons été étomné ensuite de voir cette petite espece dans l'océan Austral avec tous les mêmes caractères.

Et si une espèce est répartie dans les deux hémisphères, les autres, sans en excepter la Megaptera, ne peuvent-elles pas être dans le inême cas?

Nous commaissons déjà le Cachalot qui hante les deux hémisphères, comme plusieurs Ziphiö̈les et certains Cétodontes; parmi les Ziphioides nous pouvons citer, outre le Cachalot, le Ziphius cavirostris comme le Iicropteron Sowerbyi (Oulodon); parmi les Cétodontes, le Globiceps melus et l'Eudelphimus delphis.

Partout où, jusqu'ì présent, on a réuni des observations sur les espèces de Balénoptères, nous le répétons, on a remarqué qu'il y a quatre formes, différentes par la taille et par les caractères extérieurs et intérieurs, et qui correspondent aux quatre formes de l'Atlantique septentrional.

1H. Jovan, La chasse et la péche des animaux marins, Paris. 
Le capitaine Scammon parle de quatre Balénoptères au nord de la Californie, dont la petite, comme nous renons de le dire, a tous les caractères de notre petite espèce, et la grande, ceux de notre Balcenoptera Sibbaldii. La petite espèce y est désignée sous le nom de Balcenoptera Davidsoni, la grande, sous celui de Sulphurbottom (Sibbaldius Sulfureus, Cope).

Cette dernière se trouve aussi bien dans l'Atlantique que dans le Pacifique, dit le capitaine Scammon.

La Balienoptera musculus y porte le nom de Bulanoptera velifera.

M. Burmeister a recueilli, pour son Musée de Buenos-Ayres, des squelettes qui se rapportent égalenent à trois de nos espèces.

Mon fils avait remarqué trois squelettes de différentes Balénoptères au Musée de Buenos-Ayres, et avait reconnu l'analogie qu'ils présentent avec ceux des Balénoptères de nos parages 1. La grande espèce correspond à notre Balcenoptera Sibbuldii; elle y est désignée sous le nom de Balcenoptera intermedia; la Balcenoptera musculus y est représentée par la Balunoptera patachonica, et la troisième, la Bulcenoptera rostruta, par la Bulenoptera boncerensis.

Les squelettes du cap Horn qui se trouvent aujourd'hui au Muséum de Paris se rapportent aussi parfaitement à nos espèces européennes.

Nous en dirons autant pour les squelettes que M. Anderson a réunis pour son Husée à Calcutta.

M. Anderson distingue trois espèces de Balénoptères dans la baie de Bengale : outre la grande, de 81 pieds (Balenoptera indica), il en connaît une de 60 pieds (Balcenoptera blythii), et une de $\mathbf{4 0}$ pieds (Balanoptera edenii).

Il est question aussi d'une tête et de vertèbres d'un animal de 30 pieds, conservées au Musée de la Société asiatique ‥

1 Si le sternum de la Balcenoplera rostrala a des caractèrés particuliers, ces caractères ne sont que dé exagérations de dispositions qui se trourent déjà dans notre Balcenoplera rostrala.

- Calculta asiatic Society's Museum. Tête et vertèbres d'une Balénoptère de 50 pieds de long (jar G. Swintox, 1856) 
Ainsi ces quatre formes se retrouvent également dans la mer des Indes, et parmi elles figure l'espèce qui dépasse 80 pieds comme notre Balcenoptera Sibbaldii.

M. Anderson accorde à la grande 84 pieds et fait mention d'un individu capturé en 18501 à la latitude de $19^{\circ} \mathrm{N}$, on Juggu or Amherst Islet, qui porte au Musée de Calcutta le nom de Bal. inclica. On a cité un individu de la même taille dans la mer Rouge.

Dans ces dernières années, le Muséum de Paris a reçu des squelettes du Japon, que nous avons pu comparer avec les nótres, et nous n’avons aucun doute sur la présence de la petite et de la grande espèce dans les eaux du Japon, la Balcenoptera rostruta et la Bulchoptera Sibbuldii. Le Muséum a reçu également du Japon une tête et des fanons qui se rapportent fort bien à notre Bulcenoptera musculus. Quant à la quatrième espèce de ces mêmes parages, nous en comnaissons depuis longtemps les squelettes, dont on a fait la Balcenoptera Schlegelii.

Nous trouvons également quatre formes dans l'océan Austral, qui correspondent parfaitement aux nottres par la taille comme par les autres caractères.

M. James Hector 1 et d'autres naturalistes de la NouvelleZélande ont fait la même observation sur ces Balénoptères, et, depuis longtemps, nous avons fait remarquer que la petite Balénoptère (Balcenoptera huttoni) de la Nouvelle-Zélande a tous les caractères de notre petite espèce.

A Melbourne, on conserve, dans les collections de l'Université, les fanons et le squelette d'une Balénoptère de 90 pieds, qui a été jetée sur la côte de Vittoria. Les fanons sont noirs, larges de 18 . pouces et longs de 28 (Giglioli). Ce sont bien les caractères de notre grande espèce.

Sur la côte sud-est d'Otugo, une autre Balénoptère de très grande taille a échoué en 1873. Le capitaine Hutton en fait mention dans les Transactions de la Nouvelle-Zélande (vol. VII).

II. Kinox fait mention de trois espèces de tailles différentes,

1 Trans. New Zealand Institule. 
dans les eaux de la Nouvelle-Zélande, qui correspondent parfaitement aux nôtres: une de 80 à 100 pieds, que l'on a désignée sous le nom de Rorqualus major, une de 30 à $30 ̈$ pieds, le Sulfur-Bottom, et une troisième de 25 à 30 pieds, le Rorqualus minor 1.

J. von Haast a fait mention de la petite Balénoptère, dont un mâle a échoué le $S$ février 1880, on the Summer Beach, et qu'il n'hésite pas ì rapporter à notre Balcunoptera rostrata. Du reste, nous en avons pu comparer un squelette qui est conservé au British Museum.

Les squelettes et les ossements séparés de la NouvelleZélande, reçus dans ces derniers temps au British Museum, confirment complètement ces appréciations.

Le capitaine F. WV. Hutton, en parlant de la flore et de la faune de la Nouvelle-Zélande, dit que l'une et l'autre sont si particulières sous ces régions australes, qu'elles forment une province distincte du reste $\mathrm{du}$ monde; cela peut être vrai pour les plantes et les animaux terrestres, mais cela n'est pas exact pour les animaux marins, pas plus pour les Cétacés que pour les poissons. Nous venons de voir les mêmes Balénoptères également au nombre de quatre, et à celles-là, nous pouvons ajouter le Cachalot, le Ziphius cavirostris, le Micropteron Sowerbyi, le Globiceps melas, les Eudelphimus delphis, auxquels, il est probable, nous pourrons en ajouter bientôt d'autres. Nous sommes loin de l'époque où le capitaine Hutton écrivait : sur treize Cétacés de ces parages, se divisant en six familles, les deux tiers appartiennent exclusivement à la NouvelleZélande.

Le Challenger a rapporté diver's ossements recueillis au milieu du Pacifique, dans une station remarquablement riche en restes de Cétacés 2; parmi eux se trouvent plusieurs caisses tympaniques, et le professeur Sir Turner n'a pas hésité à les rapporter,

' Krox, Proc. New Zealand Institute.

2 Station 286 , lat. 53029' S, long. 135029' W., 16 octobre 1875, à $2,353^{\circ}$ brasses. 
les une's ì la Balconoptera rostrata, les autres ì la Bulcenuntera Sibbuldii. Il y a des os d'une troisième espèce encore indéterminée.

Et ce n'est pas seulement à l'époque actuelle que nous royons ces différentes formes se répéter dans la mer de nos antipodes comme ailleurs; si nous remontons de l'époque actuelle à celle qui nous a précédés, nous voyons le même phénomène se reproduire; à la fin de l'époque tertiaire, ces quatre formes principales ont laissé leurs ossements dans le Crag des environs d'Anvers, et les ossements fossiles recucillis dans les environs de Buenos-Ayres offrent la plus complète ressemblance avec eux 1. Ion fils a rapporté des vertèbres avec une caisse tympanique que l'on ne saurait distinguer de celles de notre Crag.

Dans les Enchainements du monde animal, nous trouvons une observation semblable faite sur des Mammifères terrestres : en parlant des Hyènes, II. Albert Gaudry fait remarquer que l'Hyine tuchetie des temps actuels correspond à l'Hyena perrieri du Pliocine, l'Inyene brune, à l'Hyena eximia de Pikermi, et l'Hyène rayée, à l'Hyena arvernensis de Perrier.

Lés Balénoptères sont peu hantées par les commensaux et les parasites; elles n'hébergent ni des Cyames ni des Cirripède's; on ne trouve sur elles qu'un Copépode du genre Penella, sertie par la tête dans l'épaisseur de la peau, et un autre Copépode, du genre Balanophilus, sur les fanons.

Comme parasites, nous ne connaissons qu'un Echinorhynque assez commun dans l'intestin, et nous possédons un Botriocephale recueilli également dans l'intestin, mais dont nous ne connaissons pas encore le Scolex.

Il est assez remarquable que la Baleine franche ne se couvre que de Cyames, la Mégaptère de Diudema, les Balénoptères de Penella.

I Bulletin de l'Acallémie royale de Belgique, 2e série, t. XXXV, 187̄̄, p.77ö. 


\section{BALENOPTERA ROSTRATA.}

\section{LITTÉRATURE.}

Chemnitz, Von der Balcrua rostrata. Berlin, Beschäftign, 4 Jahr., 1779. otb. abriclus, Fauna grocnlandica. Hafniæ et Lipsiæ, 1780.

John. Inunter, Observations on the structure and œconomy of Whales. Philos. Transactions, vol. 77, 1787.

Xeil, Some account of a fin Whale (Bal. acuto-rostrata), MeM. Wern. Nat. HIST. Soc., I, 1811.

Knox, Account of the dissection of a young Rorqual (Bal. rostrata), Jourial L'INSTITUT, 1854 , p. 556.

Kröyer, Bemarkn. Om Balcena rostrata, Kroyer's nalurh. Tidsskr., 1859, p. 617. Isis, 1841, p. 429.

Eschricht, Die Nordische Wallhiere, 1849.

Gratiolet, Comptes rendus, 1861, vol. 52, pp. 622, 891 et 942.

w. I. Flower, On a Lesser Fin-Whale. P'roc. Zoou. Soc., 1864.

Barker and Macalister, Proc. of the Dublin nal. hist. Soc. for., $1860 \%$.

A1. Carte and Macalisten, Philosophic. Transactions, 1868.

Perrin, Noles on the Anatomy of Balanoptera rostrala. PRoc. ZooL. Soc., décenibre, 1870.

Van meneden, Une têle de Balcinerelivece du fond de la merdu Nord. JourNaL DE ZOOLOGIE, t. IV, 1875.

Capellini, Sulla Balenoplera Mondini, in-4. Bologne, 1877.

TONE XLI. 
Sars, Bidrag til en noiera charact. af vore Bardehvaler. Christiania Videnskabs. Forhandl., 1878.

r. Collett, Bemerlininger til Norges Pattedyrfauna, Nyt Mag. f. Nuturvideskaberne, 1876.

R. Collett, Bidrag til Norges Pattedyrfauna, ib. 1882.

Gervais, Remarques sur l'analomie des Célacés.. Nouv. Archives DU MUSÉUM, t. VII, pl. III.

Charles Julin, Recherches sur l'ossification du maxillaire inférieur et sur la constilution du système dentaire chez le folus de la Balcenoptera rostrata. Archives de Biologie, vol. I, 1880.

Julius von Hast, Noles on Balcenoplera rostrala, fabr. (B. IIuttoni, Gray), Phil. Institute of Canterbury, décembre 1880.

Julius von Fasst, On Balcenoptera IIuttoni, Gray, Trass. Proc. New. Zeal. Instit., 1880, vol. 15.

Capt. C. M. Scammon, On a New Species of Bulconoptera, Bal. Davidsonii. Proc. CaL. Acad. of Sc., octobre 1872.

Van Beneden, Une nouvelle Balienoptera rostrata dans la Méditerranéc. Bulletins de l'Académie royale des sciences de Belgique, décembre 1884.

Beauregard, Note sur une jeune Balcenoptera capturée près de Fécamp. Conptes rendus, Soc. de Biologie, tome I, 27 novembre 1885. 


\section{HISTORIQUE.}

La Balcenoptera rostrata de Fabricius, qui n'est pas la Balcena rostrata de Linné, comme nous le verrons plus loin, est connue depuis les temps les plus reculés; il en est fait mention dans les plus anciens manuscrits des Islandais. Le mot Tiliagulik, sous lequel les Esquimaux la désignent, correspond assez bien avec celui de Tschilagulik, que les habitants de l'Amérique russe donnent à une petite Baleine du détroit de Behring; cette analogie de noms est intéressante à plus d'un titre : elle semble indiquer des relations anciennes entre les populations du Groënland et celles qui occupent aujourd'hui les régions de l'Alaska; elle montre clairement, en outre, que les habitants des côtes de l'Amérique russe ont cru retrouver, dans la petite Balénoptère du détroit de Behring, le même animal qui vit dans les eaux du Grö̈nland et que Fabricius a fait connaître sous le nom de Balcenoptera rostrata.

Mais si les pêcheurs distinguaient bien cette espèce des autres Balénoptères, il n'en était pas de même des naturalistes. La confusion la plus complète a régné dans les livres, jusqu’au jour où l'on a commencé à conserver les squelettes de ces animaux en vue de pouvoix les comparer.

Cuvier et surtout Eschricht ont été les premiers à reconnaître que la connaissance des Cétacés n'était possible qu'à cette condition; les matériaux qu'il reçut du Groënland permirent à Eschricht d'aftirmer que la Balcenoptera rostrata de Fabricius n'est pas la Balcenoptera rostrata des auteurs; il put définir nettement les caractères distinctifs de l'espèce et empêcher ainsi toute confusion ultérieure.

Linné n’a pas connu cette Balénoptère; mais 0 . Fabricius, pendant son séjour au Groënland (1768-1775), ayant eu l'occasion de l'étudier et croyant reconnaître en elle la Balona rostrata de Linné, l'a désignée sous ce nom. C'était une erreur de la part de Fabricius, mais le nom qu'il a attribué à cette espèce de Baleine lui est resté. 
Fréd. Martens a parlé ensuite de cet animal sous le nom de petite Baleine (kleine Walvisch); mais c'est à John IIunter que l'on doit les premières observations anatomiques. Le savant anatomiste anglais a eu l'occasion de disséquer une femelle, qui avait été capturée au Doggersbank dans la mer du Nord; John Hunter a fort bien reconnu que cette petite Baleine est la même que 0. Fabricius avait connue au Groënland.

Bonnaterre a fort bien caractérisé cet animal d'après les écrits du savant missionnaire danois, et Lacépède en a parlé également sous le nom de Bulénoptère à museaupointu; Lacépède avait connu un jeune animal qui avait été pris, en 1791, dans la rade de Cherbourg. C'est la moins grande des Balénoptères, dit Lacépède, et elle ne parvient qu'à une longueur de 8 à 9 mètres. La gravure, dont Lacépède accompagne le texte, est faite d'après un dessin que sir Joseph Banks lui avait envoyé de Londres.

Cette figure de Lacépède représente un gonflement extraordinaire de la langue et de la cavité de la bouche, dû sans doute à la poche du larynx que l'on a comparée à une vessie natatoire.

A l'époque où Cuvier s'occupait des Cétacés, on ne possédait aucun ossement de cette espèce dans les collections, et la Balénoptère à museau pointu fut considérée à tort comme un animal n'ayant pas atteint toute sa croissance. On voit clairement ici les services que les collections doivent rendre à la science. Les directeurs des Musées sont les conservateur's des archives qui sont mises à la disposition du public.

A la séance du 21 avril 1834 de la Société royale d'Édimbourg, le $\mathrm{D}^{\mathrm{r}}$ Knox fit un rapport sur la dissection d'une jeune Balcenoptera rostrata, et ajouta des observations fort intéressantes sur l'anatomie d'un foetus de Mysticetus 1.

M. Knox eut l'occasion d'étudier un jeune animal de 9 à 10 pieds de longueur, capturé près de Queensferry (Baie du Forth); il reconnut facilement, par le nombre de ses vertèbres, l'espèce que 0 . Fabricius avait désignée sous le nom de rostrata.

1 Journal l'Institut, 1834, p. $35 \overline{6}$. 
Depuis le siècle dernier, on possède ì Bologne une tête bien conservée, dont Mondini et d'autres avaient fait mention.

Le professeur Capellini en a fait l'objet d'un travail spécial et il a cru devoir en faire une espèce nouvelle, qu'il a dédiée à Mondini.

Postérieurement aux recherches du professeur Knox, d'Édimbourg, Eschricht recut de son ami Holböll. gouverneur du Groënland, des matériaux immenses se rapportant ì la plupart des animaux marins qui visitent la côte du Groënland, et parmi lesquels se trouvaient plusieurs squelettes d'adultes et sept fotus, dans l'alcool, dont deux mâles et cing femelles de la Balcmoptera rostratu; ces fotus variaient en longueur depuis 8 jusqu'à 78 pouces.

Dès ce moment la cétologie entre dans une phase nouvelle; ce qu'on ne peut voir dans le ventre ou la poitrine d'une Balénoptère, sans le secours d'échelles et de marchepieds pour arriver aux viscères, on peut le disséquer dans son cabinet quand on a un fœetus sous la main; aussi Eschricht démontre d'abord que, sous le même nom, on a désigné des espèces bien différentes les unes des autres, que la $B$. rostrata ne dépasse pas 30 pieds de longueur, que Fabricius l'a très bien connue, qu'elle n'a pas plus de 48 vertibres dans sa colonne vertébrale, que son sternum est en croix latine, que ses fanons sont de couleur jaune, et que la nageoire pectorale porte un cherron blanc sur un fond noir.

On peut dire que c'est depuis les travaux de l'illustre cétologue de Copenhague que cette Balénoptère a été définitivement reconnue.

En 1881, nous avons fait commaître qu'une nouvelle Ballenoptera rostrata venait d'être capturée dans la Méditerranée.

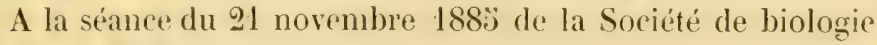
de Paris, M. Beauregard a communiqué une note sur une jeune Balénoptire, capturée près de Fécamp. C'est le mème animal dont j’arais entretenu l'Académie à la première séance du mois de septembre. La Balénoptère de Fécamp ist une femelle de $3^{\mathrm{m}}, 7 \ddot{\dot{y}}$. 
Le Muséum de Paris a reçu un très bon moulage de cet animal, et son squelette est conservé au Musće du Havre.

M. Perrin, démonstrateur d'anatomie au King's College à Londres, a décrit une jeune femelle, capturée en avril 1870 à Neumouth, dont la longueur était de 13 piers $8 \mathrm{H} / \mathrm{q}$ pouces.

Il ajoute deux dessins représentant les nageoires pectorales avec leurs muscles en place, vues du côté dorsal et du côté opposé 1.

M. Julin a publié un travail fort intéressant sur l'ossification du maxillaire inférieur et la constitution du système dentaire du foetus 2.

Au mois de novembre 1860 a échoué, au sud-est de Crower, une Balénoptìre de 2ơ pieds de longueur, que le professeur Flower a fait comnaître 3 . C'était un mâle. Son estomac était plein de débris de poissons, qu'il croit être des cod-fish (Gades). Il était presque adulte, à en juger par les épiphyses. Tout le squelette est décrit avec soin par le savant Directeur du British Nuseum.

II. James Hector fait mention de deux têtes provenant du nord de Cooli street et qui ressemblent, dit-il avec raison, à celle de la Balienoptera rostratu. Il accorde 7 pieds de longueur à la mandibule. C'est la Balcenoptera IInttonii de Gray.

Dans le courant de l'année, M. Guldberga publié un mémoire intéressant sur la biologie des Balénoptères du nord atlantique; ce travail renferme plusieurs observations intéressantes sur cette espèce. Nous avons mis ces nouveaux faits à profit, notamment dans le chapitre où nous traitons du genre de vie et des phénomènes de la parturition.

Le professeur Burmeister a réuni, dans son Musée de BuenosAyres, les squelettes de différentes Balénoptères, parmi lesquelles nous en trousons une petite qui a tous les caractères de notre petite espèce si bien décrite par Fabricius.

1 Pernin, Notes on the anatomy of Balanoptera rostrata. Proc.zool.Soc., décembre 1870.

2 Archives de Biologie, vol. I, 1880.

${ }^{3}$ On a lesser Fin-Whale (Balcenoptera rostrata, Fabr.), recently stranded on the Norfolk coast. Proc. Zool. Soc, may 1864. 
En 1873, une Balénoptère de 16 \% $/ 2$ pieds, ayant 48 vertèbres, les fanons blancs et le sternum en croix, a été capturée sur les côtes de la Nouvelle-Zélande (Otago Heads). Le professeur Hutton en a donné une figure et, plus tard, le professeur von Haast en a publié une description en joignant à la description une figure du sternum. Gray lui avait donné le nom de Balcenoptera Inttonii, et J. von Haast a rapporté ce même animal à notre Balcenoptera rostrata. Il résulte de ces faits que la petite Balénoptère, à 48 vertèbres, habite également les deux hémisphères.

Le capitaine Scammon a décrit une petite Balénoptère du Pacifique, sous le nom spécifique de Davidsonii qui, dans notre opinion, est synonyme de Balanoptera rostrata.

\section{SYNONYMIE.}

Cette espèce, la plus petite de toutes, est désignée sous les noms les plus différents. Nous pourrons les énumérer ainsi :

Balana rostrata, 0. Fabr.

Balcenoptera aculo-rostrata, Lacépède.

Rorqualus minor, Knox.

Pterobalana minor, Eschricht.

Balcenoptera rostrata, Gray.

$\begin{array}{ll}\text { - } & \text { Esclirichtii, Rash. } \\ \text { - } & \text { Davidsonii, Scammon. } \\ \text { - } & \text { Mondini, Capellini. } \\ \text { - } & \text { Boncerensis, Burmeister. } \\ \text { - } & \text { de Huttoni, Gray. }\end{array}$

Cette même espèce est encore désignée par des noms vulgaires :

Baleine d'été. Côte de Norwège.

Piked Wahle. Pennant.

Lille Finner Pike Whale des baleiniers anglais.

Tikagulik, des Groënlandais.

Vaagehval, des Norwégiens.

Zwergwhal, des Allemands. 
La Balenoptera Datidsonii de Scammon est bien, comme il le soupsonne, semblable ì la Balienoptera rostrata d'Europe, quoiqu'elle habite les coites de Californie. "This species is evi"dently congeneric with the Bulunoptera rostratu ) dit arec raison le capitaine Scammon 1.

La troisieme Balienutera musculus de Pallas, qui nat que 221 . pieds de longueur, est sans doute une Balienoptera rostrata.

La Balénoptère à museau pointu de Lacépède pl. VIII! est bien l'espèce désignée sous le nom de Balcenopteru rostrata.

La Balcenoptera Huttonii de Gray n'est qu'une rostrata.

La Bulenupteru rustrutu est regardée, sur les côtés de Massachusetts, comme une jeune Balcenoptera musculus.

\section{CARACTÈRES.}

Il n’̣ a pas d'espèce plus facile à caractériser ; elle ne dépasse guire 30 pieds de longueur, quoiquon en ait ru de 36 ; la nageoire pectorale a un cherron blanc; les fanons sont toujours de la méme couleur jaune pàle; la colonne vertélorale compte 48 vertibres; le sternum est en croix latine; les crites sont au nombre de 11.

On la reconnait toujours parfaitement ì l'extérieur au chevron blanc qu'elle porte sur les nageoires pectorales.

Dans un certain nombre de squelettes, on voit des coalescences entre les corps ou les apophyses de quelques vertèbres, le plus souvent entre les cervicales; mais ces dispositions n'ont aucune valeur sous le rapport systématique.

Parmi les individus qui sont venus à la côte, nous en trourons un qui n’a que de 9 à 10 pieds, cinq qui ont de $1 \ddot{3}$ à 17 pieds, quatre de 24 à 20 pieds et un seul de 29 pieds. Le premier, de 9 à 10 pieds, vient de naitre, et les autres, de 14 à 103 pieds,

1 Cap Scaysox, On a new species of Balcenoptera. Proc. of the cal. Academy of sciences, octobre 1872. 
cest-ì-dire la moitie de la taille de la mire. viennent sans dout: de la quitter. Les jeune se siparent de la mare a lâge de deux ans. Ils ont enerre la moitie à geagner pour atteindre la taille adulte.

Le squelette le plus fort que nous arons ru, est celui du

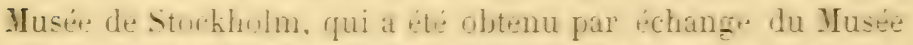
de Bercen. Ln antre squelette diun animal tres fort se troure au Musée de l'Lniversité de Liège. Il vient également de Bergen.

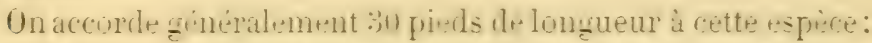
mais, à en juger frar la longuedur du jeune en venant au monule. longueur qui ent de preds dapres Eschricht, cut animal,

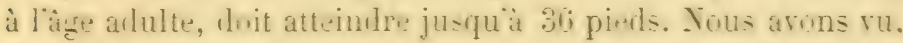
du reste, certains squmettes qui confirment cette dimension comme taille naturelle.

\section{ORGAXISATION.}

I. Charles Julin a musione, dans les Arehives de Biglogeie, de's observations du plus hat interet sur la constitution du systeme dentaire diun lietas de cette espiece. Les dents rappellent plus ou moins les dents arlultes des sifualoulons.

Sous avons compti: dans un fietus quatre bulbes pileux d la màchoire superieure et cing à la màchnire interieure.

La colonne vertibrale so compose géniralement de 7 cervicales. 11 dorsales. 133 lombaires et 17 caudales, en tout 48 . Il y a quelques squelettes dans lesquels il y en a pluset dautres, en plus grand nombre, où il y en a moins.

Dans le mâle du Musere royal du college des chirureiens ì Londres, on woit les apmphyse transverses, de la troisieme it la sixième cervicale, séparées, ne pas former un anneau complet.

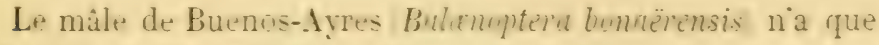
32 pieds et 48 vertèbres comme notre espèce.

Dans le squelette de Hunter, les apriphrses transverses supérieure et inférieure de laxis ne sont pas non plus reunies. 
La sixieme cervicale de l"individu de Yorfolk Coast, conservé au Musere roval un College des chirurgiens, a un anneau complet d'un côté, incomplet de l'autre.

Il y a ausi parfois cualescence entre les corps de deux rertejnes qui se suivent. Nous en arons ru dans les régions cerricale et caudale.

Lous arons ru de's squelettes qui ont le corps de l'axis soudé à la troisiome cervicale. C"est ce que lon ubserve dans un squelette du Colle ge des chirurgitens, ei dans un autre, du Colonial Museum de Wellington (Nouvelle-Zélande).

Les trois dernieres caudales sont écalement réunies dans un squelette que nous arons eu sous les yeux.

Xous avons deja fat la remarque que la Balonuptera boreculis montre habiturllement la première crite bifide; nous en avons cite un eas remarquable, en 1 sitis, dans les Bulletins de liAcadimie : dans le squelette de Balenoptere restrata du Musée de Cambrilge, nous royuns éralement des traces de fusion des deux premières côtes.

Cotte bitidité de la première crite a souvent été vue chez Ilomme: elle a ete signalee dans un squelette de Glubiceps melus du Iapon, qui est a Letede, et dans un Delphinapterus leueas du Musin du College royal des chirurgiens de Londres. Le cas le plus interenant est celui que nous arons signalé dans un natsouin Phacona communis. La cite supp'émentaire est déreloppée des deux côtés?

Le sternum ent caractiristique par sa forme en croix latine. La Buhnmitern buntrensis:" de Burmeister présente cette mème forne. Inais prestnte en cutre, en avant, deux saillies formant une sorte de fourche que nous avons retrouvee en miniature dans des sternums très adultes.

Le sterrum de la Balemutera boreulis, à lầge foetal, présente citte nène fourche, sans arvir le bout xiphioilde allongé.

1 ge série, t. XXVI, n०7.

= Bulletins de l'Académie, 2e série, 1. XXVI, no $\%$.

- Atlas de la descriplion physique de la république Arzentine, pl. $\Gamma$, fig 5. 
Les fanons de celte expere sont toujours faciles à reconnaitre à leur longueur ainsi quä leur conleur jaune pàle. Les plus longs fanons ne dépassent pas 2 pieds.

\section{MOEL'RS.}

Le capitaine Holböll a eu loccasion dobserter cettie petite Balengptere sur la cite du cromenland, et il fait remarquer quon la roit au milieu des erandes Baleines. Il nen est fras de meme de la Babneqtera muculus, dont les baleiniers considerent lapparition comme un indice certain de la tin te la saison de pèche.

Quand un ne les wit pas au milieu de grandes Baleines, ces Balénoptères sont isolées ou à deus et trois ensemble.

Comme lrs autres Balenupteres virent par couples et qu ä Bereen on woit souvent des males et des femelles, dint les dernières seules approchent des cûtes, il y a tout lieu de croire que l'espèce qui nous occupe mène le mène genre de rie.

Cette petite esperce poursuit les puissons comme la $B$ aldantera musculus et borealis.

Hunter a trouve dans lestoma des restes de divers poisoons, surtout du Dog-fish.

Lne femelle arture à Weymouth arait l'estomae vide: il y arait au lieu de fatture dix cailloux dans son fremier estrmae et autant dans le second (Perrin).

Motzfeld a vu à Juliane haab, côte du Groënland, cette Balenoptere avaler des Madotus ardicus, fermer la bouthe au-dessus de l'eau et rejeter ensuite leau de la bouthe en jets l'ecume des deux crites: puis, apres un moment de repas. il a vu lhaleine sortir des narines comme clez tout autre animal qui respire.

On a ru souvent cette Balénoptire entoure de Tursiops tur.ie, au moins au nord de l'Atlantique. Ils poursuivent sans doute la même pàture. 
D'aprìs Eschricht la gestation n'est que de dix mois; en naissant l'animal a 9 pieds de long, c'est-à-dire, comme dans les autres espèces, à peu près le quart de la longueur de la mère.

Melchior a vu un foetus de 8 pieds 2 pouces qui nlétait pas à terme.

On a recueilli à Bergen des fœtus de différentes tailles, et les femelles arrivent cependant à la mème époque de l'année.

Eschricht a vu plusieurs exemples de jumeaux.

Sur les côtes de Finmark on recueille également des foetus de tailles différentes à la même époque.

En faisant le releré des côtes où des individus sont venus échouer et où on a tenu compte des dates de leur capture, on ne peut pas dire qu'il y ait quelque part un passage régulier, si ce n'est sur la côte de Norwège et à l'entrée de la mer' de Battin. Nous commaissons leur apparition périodique dans les Fiords de Bergen en été, comme dans le détroit de Davis, mais nous ignorons completement, comme pour les autres Balénoptères, le lieu de leurs quartiers d'hiver.

Il est à remarquer qu'il n'y en a pas une seule, de celles qui sont venues vagabonder sur nos côtes tempérées, qui ait dépassé ou même qui ait atteint 30 pieds.

Grâce aux nombreux fœtus que l'on a pu recueillir, en tenant compte de la date de la capture de la mère et de la taille du fœtus, en faisant ensuite la comparaison des jeunes animaux capturés pendant les différents mois de l'année, on a pu constater de combien par mois les foetus grandissent dans le corps de la mère et de combien les baleineaux s'accroissent par mois pendant la première année de leur vie.

On a recueilli un grand nombre de fotus et on a heureusement tenu compte de la date de la capture de la mère et de la taille des fœtus. Eschricht est arrivé à ce résultat, confirmé par Guldberg, que le développement commence dans les premiers mois de l'année et continue jusqu'en novembre; en avril il a reçu un fœtus de $0^{\mathrm{m}}, 090$ et en septembre un autre de $1^{\mathrm{m}}, 624$. 
Comme la taille, à la naissance, est, d'après Eschricht, de $2^{\mathrm{m}}, 8$, il y a lieu d'en conclure que la mise-bas a lieu en hiver.

Le Dr hinox a signalé un jeune animal capturé au mois de férrier 183.4 qui arait 9 pieds 11 pouces. C'est un peu plus que la taille du baleineau au moment de la naissance. Il avait probablement un peu plus de deux mois.

Le 18 février ou mars 1878 , un individu long de $3^{\mathrm{m}}, \ddot{0} 0$ a été capturé près de Villefranche.

En férrier 1861, un de 3 mètres a échoué sur les côtes de Bretagne.

En arril 1791, on en a pris dans des filets, près de la rade de Cherbourg, un individu qui avait 14 à $10 ّ$ pieds.

La jeune femelle que M. Perrin a décrite a été capturée en avril 1870; elle était longue de 13 pieds 8 1/2 pouces.

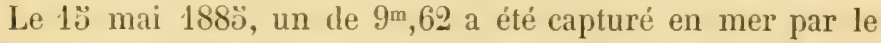
Gaulois, de Fécamp.

Le 27 septembre 1863 , un de $S^{\mathrm{m}}, 60$ à Saint-Jean-de-Luz.

Le $1 \ddot{3}$ septembre 1878, un Vaagerhal a été pris dans le Fiord de Christiania ; il avait $14 \mathrm{1} / 2$ pieds.

En nove'mlre 1860, un mâle de $20 ̈$ pieds a échoué au sud-est de Crower (Flower).

D'aprés un manuscrit sur les pêches, cité par II. Guldberg, le temps de la mise-bas du Vaagevhal serait le commencement de novembre. Guldberg croit que cette éporque est un peu trop arancée, et la fixe entre la fin de novembre et le commencement de janvier.

La gestation serait, comme Eschricht l'a estimée, de dix mois.

Laccouplement aurait lieu pendant les premiers mois de l'année.

Rieste la question de saroir ou ils se réfugient pour mettre bas et de comnaitre les lieux où ils s'accouplent. 


\section{DISTRIBUTION GÉOGRAPHIQUE ET PECHE.}

Pendant l'été on les roit communément sur les côtes de Finmark, mais on ne les y chasse généralement pas.

Guldberg a trouvé tout près de Vadsö, dans le Varangerfiord, le squelette d'un individu qui s'y était perdu pendant l'hiver.

Nous allons citer d'abord les parages où des individus sont venus se perdre, en faisant remarquer que les Cétacés n'échouent généralement, comme l'a dit Eschricht, que sur les côtes qu'ils ne fréquentent pas régulièrement.

Le plus ancien exemple connu date du XVII ${ }^{\mathrm{e}}$ siècle. Le 8 mai 1699, une petite Baleine vient se perdre dans le Weser et son squelette est conservé à Brême. La ville de Brême en a fait exécuter une peinture à l'huile. Cuvier en parle, mais en prenant cet animal pour un jeune Rorqual; le grand naturaliste n'avait pas de matériaux pour débrouiller cette histoire.

Nous en connaissons deux exemples en Belgique.

Le 10 juillet 1838, une jeune femelle de $̋$ mètres a été trouvée morte en mer près d'Ostende. Son squelette est au Musée de l'Université de Gand.

En 1868, au mois d'octobre, un mâle de 16 pieds a remonté l'Escaut et s'est fait prendre en amont d'Anvers. Son squelette est conservé à Bruxelles, au Musée royal.

Sur les côtes océaniques de France on a vu se perdre plusieurs individus.

En avril 1791, un jeune Rorqual de 14 à 103 pieds de long, dont le milieu des nageoires pectorales était blanc, est venu se perdre dans des filets de pêcheurs, près de la rade de Cherbourg. Un médecin de Valogne en a envoyé une description à Lacépède, et c'est d'après cet animal que ce naturaliste a établi la Balénoptère à museđu pointu. La bande blanche des nageoires pectorales, dont parle le médecin de Valogne, ne laisse pas de doute sur l'espèce à laquelle appartient ce jeune animal. 
Le 10 mars 1827, un individu de 7 mètres de long a été capturé sur les côtes d'Oleron; nous en avons vu les ossements au Musée de la Rochelle.

Le Dr Fischer croit que c'est par erreur qu'on a annoncé la capture d'une petite Balénoptère en juin 18300 sur les côtes du Morbihan; mais il n'est pas douteux qu'en 18302 on en a pris une à l'embouchure de la seine, et dont le dessin est conservé dans les Vélins du Muséum 1.

Le 26 août 1833̈, un mâle a échoué dans la Charente; son squelette est conservé au Musée de l'École de médecine de Rochefort; sa longueur était de 7 mètres 48 centimètres.

Un autre individu a été capturé sur la côte de la Gironde, dont le squelette n'a pas été conservé ( $\mathbf{F}$ ischer).

Au mois de février 1861, un individu de 3 mètres a échoué sur les côtes de Bretağne.

Vers 1879, M. Quillau a envoyé un squelette incomplet au Muséum de Paris, qui provenait sans doute de cet animal.

Un autre encore, de 6 mètres 60, a échoué à Saint-Jean-deLuz le 27 septembre 1863.

Au Musée de Lille on conserve le squelette d'un individu échoué sur les côtes de Montreuil-sur-Mer (Fischer), et un autre à Brest, provenant d'un animal reconnu par Rochon.

A Bordeaux on conserve le squelette d'un animal qui a échoué à Boulogne.

Le 100 mai 188ə̃, un bateau de Fécamp (Le Gaulois, patron Deshayes) a capturé en mer une Balchoptera rostrata, longue de 3 mètres 62 pieds. M. Leunier, directeur du Musée du Havre, en a donné le dessin d'après l'animal étendu sur le pont 2 .

On a été longtemps dans le doute sur la question de savoir si la petite Balénoptère pénètre dans la Méditerranée. Ce doute est levé maintenant.

On en connaît aujourd'hui des exemples bien constatés, mais

1 Ce dessin, le plus beau que nous connaissions de celte espèce, a été reproduit par P. Gervais dans les Annales du Muséum, Mémoires, t. VIl, pl. õ.

2 La Nature, 7 novembre 1885. 
en tout cas ce sont des apparitions bien rares; un squelette est conservé depuis le siècle dernier au Musée de Bologne, provenant, paraît-il, d'un animal capturé en 1771 dans l'Adriatique; il a été décrit par Mondini, et le professeur Capellini a cru devoir lui donner le nom de Balanoptera Mondini 1.

L'abbé Ranzani avait envoyé à Cuvier un dessin de la tête, conservée au Ilusée de Bulogne, et Cuvier avait cru qu'elle était semblable à celle du Rorqual, de manière, dit-il, qu'il n'y a pas lieu de douter que la même espèce de Rorqual ne vive dans la mer du Nord et dans la Méditerranée; mais en même temps il existe dans cette dernière mer une autre espèce, celle qui a échoué aux Iles Sainte-Marguerite, et que le grand naturaliste croyait propre à cette mer intérieure.

Le 18 mars ou février 1878, un autre individu, long de $3^{\mathrm{m}}, \ddot{30}$, a été capturé par les pêcheurs de Saint-Hlospice, au petit port de Saint-Jean, près de Villefranche (dép. des Alpes maritimes) ¿2. Le corps a été acheté par les frères Gall, de Nice. Le squelette est conservé à Florence.

Nous ferons remarquer que la Méditerranée ne nossède aucun Cétacé qui lui soit propre, pas plus que la Baltique et la mer Noire, et qu'il ne pénètre même aucun Cétacé à fanons dans cette dernière mer intérieure.

Il y a eu des Cétacés ì fanons dans la mer Noire à la fin de l'époque tertiaire, en même temps que des Siréniens et même des Squalodons; mais aujourd'hui on n'y voit plus que trois Cétacés, tous les trois à dents : le Marsouin, le Tursio et le Dauphin ordinaire, qui viennent de l'Atlantique.

On connaît aussi quelques individus qui sont venus à la côte en Hollande.

Le 20 décembre 1862 , après un violent orage, une femelle de כ̆ mètres de long est allée échouer dans l'Y. Le squelette en est conservé au Jardin zoologique d'Amsterdam.

1 Gervals, Journal de zoologie, 1877, p. 167.

2 P. J. Vax Benfeden, Un mot sur quelques Cétacés échoués sur les cótes de la Médilerranée. Bullet. Acad. noy. de Belgique, février 1580. 
M. Max Weber amnonce, en 1861, qu'un animal de 30) pieds de long est venu se perdre dans la Zuiderzée, sur les côtes de V'lieland, mais il ne dit pas l'époque de l'année, ni si le squelette est conservé 1.

On connait plusieurs exemples de Balcenopter to rostrata échouées sur les côtes d'Angleterre et d'Écosse.

En 1763 les pêcheurs ont pris, dans la mer du Nord, au Doggersluank, une jeune femelle de 16 a 17 pieds; John Hunter l'a disséquée, et le squelette en est conservé dans son musée, aujourdhui le Ilusće du Collège royal des chirurgiens.

Le 14 norembre 1808, une femelle de 17 pieds a été capturée aux Orcades, dans Scalpa-Bays.

En février 1834, une jeune femelle de 9 à 10 pieds, la plus petite que l'on ait encore vue se perdre, est allée échouer dans le Firth of Forth. Son squelette est conservé au Musée d'Édimbourg. Il y a tout lieu de croire que l'animal venait d'être mis au monde.

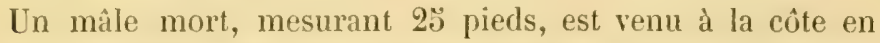
novembre 186:, près de Cromex Norfolk). Son squelette est conservé au Musée du Collège royal des chirurgiens à Londres. Son estomac était plein de cod-fish, Gudus aglefimus.

Le 8 mai 1863, un jeune individu de 10 pieds 2 pouces est venu se perdre Irish Coost off Cloger-IIead. Il n'a que 46 vertèbres. (Al. Carte et A. Macalister).

Le squelette d'une femelle capturée à Yarmouth, ainsi qu'une tête et une omoplate d'un animal qui a péri à l'Isle d'Islay en 1866, sont conservés à Cambridge.

Une jeune femelle de Balanoptera rostrata de 13 pieds et quelques pouces a été capturée en avril 1870 à Weymouth, et at été achetée par II. Gerrard. M. Perrin, demonstrator of anatomy, King's Cullege, à Londres, l'a disséquée et a publié les résultats de ses recherches dans les Proc. Zool. Soc., décembre 1870. Le premier et le second estomac contenaient des petits

1 Verslagen der Neder\%. Dierkund. Vereeniging, Tydschrift der Nederl. Vereeniging, 2 e serie, ath. j et $4,1886$. 
cailloux. Il ajoute deux dessins représentant les nageoires pectorales avec leurs muscles en place, vues du côté dorsal et du côté opposé.

Le 16 mai 1887 une jeune femelle s'est perdue à Plymouth 1. Un jeune animal de 9 à 10 pieds a été capturé près de Queensferry (Firth of Forth). A la séance du 21 avril 1834 de la Société royale d'Édimbourg, le docteur Knox fait un rapport fort intéressant sur la dissection de cet animal 2.

Lne jeune femelle de $14 \mathrm{1} / 2$ pieds est venue échouer vivante sur la côte d'Aberdeen, en juillet 1870. Son squelette est conservé au Musée d'Aberdeen, dirigé par le professeur Struthers.

Ln autre encore a échoué en septembre 1871 à Dunbar (Firth of Forth). Le professeur sir Turner en fait mention.

II. Flower a signalé, en 1880, une jeune femelle de 150 pieds qui est allée échouer sur les côtes de Cornouailles.

On en a vu périr également sur la côte de Norfolk, dit M. Southwell.

En 1837, sur la côte ouest de Jutland, près de Vardo, est venu échouer un individu de 22 pieds; son squelette a été envoyé à Eschricht. Il n'est pas complet.

A l'est du Jutland, un animal de 18 pieds est venu à la côte ('n juillet 182.4. Le Musée de Halle en possède le squelette.

On a c'mregistré aussi quelques exemples d'individus égarés dans la Baltique.

En 1840̈, un animal, dont le dessin est conservé dans l'église Sainte-Marie à Greifswald, est venu échouer dans le voisinage de cette ville (an der Wiek).

Un autre est venu à la côte à l'île de Rugen; son squelette est conservé à Breslau 3; il a 20 pieds de longueur et 48 vertèbres.

On conserve au Musée de Stockholm une mandibule trouvée

' Buakwill, The Zoologist, july, $18 \overline{7}$.

2 Journall'Institul, 1854, p 356.

s Erist Roll. 
dans la marne de l'époque glaciaire (Halland) I, que nous rapportons à cette espèce.

Nous avons attiré l'attention des naturalistes sur une tête de rette même espèce, conservie au Musée de Brème; elle a été pêchée dans la mer du Yord, et l'on avait eru pouroir l'attribuer un instant à un animal fossile. D'après les pècheurs de Brême, les ossements de ces animaux ne sont pas rares dans certains endroits de la mer du Nord 2.

Le 2 juillet 1840 , un animal de 16 pieds est venu à la côte près de Christiania.

Un autre, un mâle de $141 / 2$ pieds, est venu échouer dans les mèmes parages en septembre 1878. Le professeur Sars en a publié un dessin.

Nous avons vu une omoplate de cette même espèce suspendue dans la cour d'un des principaux hôtels de Christiania.

Il est évident que toutes ces visites sont purement accidentelles; mais il n'en est pas de même de celles qui se font dans les Fiords de la côte de Norrige, dans les environs de Bergen. Dans ces lerniers parages, on en voit arriver périodiquement, le plus souvent depuis le mois de mai jusqu'en décembre; ce sont généralement des femelles qui entrent dans les Fiords pour y mettre bas; sur onze individus quion avait eapturés dans un temps déterminé, il n'y avait qu'un seul mâle. On a remarqué du reste partout, que les màles de toutes ces espèces se tiennent au large, pendant que les femelles approchent des côtes.

Depuis longtemps on sait que la Balcenoptera rostrata entre dans ces baies et que les pécheurs des alentours les emprisonnent ì l'aide de filets. L'animal, enfermé comme dans un aquarium, est attaqué ì coups de flèches empoisonnées; il perd immédiatement, aprìs ses blessures, de son activité ordinaire et devient facilement la proie des pêcheurs. La flèche est empoisonnée par le pus de lia capture précédente et, depuis des temps

1 Angelis, Ofvers. af k. Vet. Aliad. Forh, 1867, p. 81. Erdmax, Bidr. tıll kïnned. om Sveriges Quariura Bildingar, p. 158.

2 VAX Bexedex, Journal de Zoologie, t. IY, 1875. 
fort reculés, on prépare ainsi des flèches qui ne doivent servir qu'à la campagne suivante.

Tous les ans, dit le docteur Armauer Hansen, aux mois d'arril et de mai, les pêcheurs attendent la Balenoptera rostrata à l'entrée d'un Fiord très étroit, nommé Skogsväg, à 30 kilomètres de Bergen. Dès qu'un animal est entré, ils ferment l'embouchure au moyen d'un filet et ils l'attaquent à l'aide de flèches. Ils empoisonment leurs fleches en baignant la pointe dans la chaire gangrénée et ils la laissent sécher. II paraît que la forme des ares dont ils se servent date de l'époque des anciens guerriers, les Vikings.

M. Armauer Hansen a trouvé des bacilles dans la chair gangrénée, et croit que la flèche empoisomée inocule aux Balénoptères les germes qui produisent la septicémie ${ }^{1}$.

La petite Balénoptère a été vue également dans le détroit de Davis, mais seulement pendant les mois d'été 2; on la voit paraître aussi sur les côtes d'Islande, aux îles Loffoden, sur les côtes de Finmark et, comme nous venons de le dire, périodiquement sur les côtes de Norvège. On en a vu également dans la mer Blanche; Nordenskjöld l'a même observée dans la mer' de Kara, à côté de Belugaa, et Scoresby 3 , comme Malmgren 't et Sluyter l'ont reconnue dans les eaux du Spitzberg ${ }^{5}$.

1 Armauer Haxsen, La seplicémie inoculée à des Baleines par les flèches dont se servent les pécheurs, Anchives de Brologie, t. VI, fasc. III, 1885, p. 585 .

${ }^{2}$ Celte mème petite Balénoptère, dont nous possédons un squelette envoyé par Holböll du Groënland, se trouve également plus au nord, mais en moins grand nombre toutefois que dans la partie méridionale; elle arrive au mois d'avril à Godhab, dit Holböll, et ne quilte qu'au mois de décembre. La Balcenoptera rostrata aurait ainsi, comme du reste nous l'avons fait remarquer déjà pour la Megaptera boops, plus d'une station d'été.

3 Scoresby fait mention d'un individu de $17 \%$ pieds, capturé au mois de novenbre 1808, dans la baie de Scalpa; il a été figuré par Watson. An Account of the arct.reg., t. 1, p. 485, pl. III, fig. 2.

4 Malmgren a vu, pendant son voyage au Spitzberg, des individus de cette espèce pendant le mois de mai.

${ }^{5}$ II n'est pas sans interêt de faire remarquer que Ruyter, en gagnant des 
Avant Nordenskjöld, des baleiniers norvégiens en avaient déjà signalé dans la mer de Kara.

On en a vu en abondance dans les parages de Godthaab Grönland. Tous les baleiniers s'accordent à dire que la petite Balénoptère arrive en été au détroit de Davis et à la baie de Baftin.

De l'antr' crité de l'Atlantique, on a eonstaté depuis longtemps la présence de cette mème Balénoptère; le Husée de Stuttgard a reçu un squelette de la côte du Labrador et, depuis, on a fait mention d'm animal de 18 pieds capturé dans la baie de YewYork (Dr Kay).

M. Allen cite la Balenoptera rostrata avec un signe de doute, comme animal propre à ces parages, mais ce doute est évidemment levé.

On trouve dans l'Atantique méridional, comme dans l'Atlantique septentrional, dans le Pacifique et même dans les eaux de nos antipodes, des Balénoptères qui eorrespondent à notre Buluenopteru restrutu par leur taille, par leurs fanons ainsi que par l'ensemble de leur organisation 11 y en a qui sont tellement semblables à l'espèce de nos parages, que, si on les trourait sur les côtes d'Europe, on ne songerait pas à en faire des espèces distinctes. Telle est la Balénoptère du nord du Pacifique à laquelle, comme nous l'avons déjà dit, le eapitaine Seammon a donné le nom de Balcenoptera Davidsonii. Cette aftinité n’a, du reste, pas échappé aux naturalistes américains :

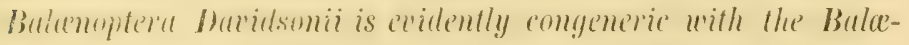
noptera rostrata, dit le capitaine Scammon. Nous avons eu l'occasion de voir à Vienne des fanons, rapportés de San Francisco par le professeur Steindachner, en tout semblables aux fanous de notre espèce naine.

A l'embouchure de la Plata du Mediano, près de Belgrano, ¿ 10 miles de Buenos-Ayres, flottait, le 3 février 1867, un

regions de plus en plus septentrionales, a vu successivenient disparaitre les Ilarsouins, puis les Tursio, et ce n'est qu'après celte disparition qu'il a vu

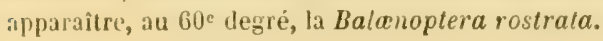


cadarre qui a été recueilli par les pêcheurs, et dont le squelette est conservé aujourd'hui au Musée de Buenos-Ayres. C'était un mâle. La taille ne dépasse pas 32 pieds; les vertèbres cervicales 2, 3 et 4 sont réunies par le corps; les apophyses transverses supérieures des sixième et septième cervicales sont réunies à gauche dans toute leur longueur. Burmeister l'a déerit sous le nom de Bulcuoptera bonärensis 1 . Il posside le squelette et en a fait connaître les divers caractères, qui sont la reproduction de notre Bulenoptera rostratu. Il n'y a que le sternum qui diffère par une bifurcation qu'il présente sur son bord antérieur, mais le squelette montre jusqu'au nombre de vertèbres (4S) si caractéristique de cette espèce.

Le British Museum a reçu également de l'île Formosa, par le consul anglais Swinhoe, des fanons, arec les barbes jaunes de la grandeur des fanons de l'espèce qui nous occupe, ou même un peu plus petits, et qui proviement sans doute de la mème Balénoptère, si pas d'une espèce similaire. Cés finons sont conservés dans un bocal; il n'y en a qu'une rangée. Nous avons fait mention de cette Balénoptère dans notre Ostéographie, sous le nom de Balcenoptera Swinhoei.

Pallas fait mention d'une Balénoptère de 22 pieds de lonģ, avee une nageoire pectorale blanehe, observée par Herle sur la côte du Kamschatka, et qu'il rapporte avec raison à la $B a-$ lanoptera rostrata de Fabricius.

Le Muséum de Paris a reçu du Japon un squelette qui a tous les caractères de cette espèce; il a deux ou trois vertèbres de plus que le nombre normal de 48 , mais on trouve de semblables variations dans des squelettes d'Europe.

Près de l'île Kerguelen, on a capturé une Balénoptère de 30 pieds de long qui appartient sans doute à cette mème espèce. On n'en a malheureusement rien conservé.

1 H. Bunmeister, Prelim. descript. of a new species of Finner whale (Ba:lænoptera bonaërensis). - Proceed. Zool. Soc., 1867, p. 707. - Annales del Museo publico de Buenos-Aires, 1868. - Vax Beneden el Paul Gervais, Osteograplice des Célacés. 
La mer de nos antipodes nourrit également, à côté de Balénoptères de grande taille, une petite espèce qui ne dépasse pas la notre en dimension; elle atteint ì peine 30 pieds de longueur. En octobre 1873, écrit-on de la Nouvelle-Zélande, on a rapturé une petite Balénoptère dont le squelette a été envoyé au British Huseum. En recerant la lettre denvoi, le docteur Gray croyait d'abord, d'après les dimensions, que c'était un squelette de Neobaluna marginata 1. Le squelette de cette Balénoptère est complet et ses fanons d'un jaune pâle (cream colour) sont conservés. Il a 48 vertèbres, un sternum en croix latine, et les fanons semblable à ceux de notre espèce. Nous ne doutons pas que, si ce squelette avait été expédié du nord de l'Atlantique, fersomne n’aurait hésité à le désigner sous le nom de rostrut».

Gray a publié le dessin que le professeur Hutton, conservateur du Muséum d'Iotago, lui a fait parvenir 2.

M. James Hector fait mention de deux têtes, provenant de Cookstrait, et qui ressemblent, dit-il avec raison, à celle de la Bulaniptera rostrata. 11 accorde 7 pieds de longueur à la mandibule.

Jul. von Haast fait également mention d'un jeune mâle, échoué on the Summer beach, le 7 février 1873 ; il a 23 pieds de long, "t le Directeur du Musée de Canterbury n'hésite pas à le rapporter à la Balcenoptera rostrata d'Europe, tout en reconnaissant que c'est la Balanoptera IIuttoni de Gray. Il représente le sternum et un fanon ${ }^{3}$.

Nous trourons dans nos notes: le squelette de la Bal(rnoptera Iluttoni, conservé au British Iluseum, a la taille, le nombre de vertibres et le sternum de notre Bulenoptera rostratu; le sternum est en croix latine, mais un peu plus petit que celui de notre rostrata. Les fanons sont jaunes, avec une bande

- En mai 1874 Gray mécrivait: "Le squelelte de la petite Baleine de Ia Xouvelle-Hollande est arrivé. C'est une Balanoptera voisine de la Balanoptera rostrata, mais bien distincte, et pas une Neobalcena. $\downarrow$

${ }^{2}$ Gray, Ann. nal. hist., 1870, vol. V, p. 224; 1874, vol. XIII, p. 516, pl. XVI.

s Philosophical Institule of Canterbury, 30 déccmbre, 1880. 
noire à l'extérieur; les vertèbres sont au nombre de quarantesix; sans doute les deux dernières manquent.

Parmi les caisses tympaniques, rapportées par le Challenger et recucillies par la sonde au milieu du Pacifique, à quelques degrés au sud de l'équateur, le professeur Sir Turner en a trouvé qui se rapportent à la Balanoptera rostrata 1.

En somme, la Balenoptera rostrata a cité observée en Europe sur les côtes du Groënland et du Spitzberg, d'Islande et de Norvège, de Suide, de Danemark, d'Allemagne, des Pays-Bas, de Bingique, de France, d'Ecosse, d'Angleterre, et enfin dans la Méditerranée et la Baltique. On l'a vue aussi dans la mer Blanche et dans la mer de Kara.

En dehors de l'Europe, elle a été observée sur les côtes du Labrador et des Etats-Lnis d'Amérique. Dans le Pacifique on l'a vur au détroit de Behring, sur lés côtes du Kamschatka, dans les parages des illes Aléoutiennes, et elle est connue sous le nom de Balanoptera Davidsonii sur les côtes de Californie.

Dans l'hémisphère antaretique on recomnaît une petite Balénoptire i 18 vertibres, sur lis côtes de la Plata, de Kerguelen et dans les eaux de la Nouvelle-Zélande.

On ne connait que deux parages où la petite Balénoptère apparaît périodiquement : ce sont le détroit de Davis et les Fiords de Borgent. De l'un comme de l'autre côté clle napparaît qu'en été. Où se troure-t-elle pendant le restant de l'année?

P'endant l'été de 1878, les naturalistes du Willem Barents ont fait quelques observations intéressantes sur les Cétacés. y. Sluyter rapporte que, an delà du $60^{\circ}$ degré de latitude, ils ne découvrirent plus de Marsouins, mais deux espèces de Dauphins, le D. (Steno ?) rostratus et le D. (Tursiops) tursio. Le premier nest pas abondant; l'autre vit par petites gammes, qui disparurent à la hauteur de l'île Jan Meyen. A l'ile des Ours et à la côte de la Nouvelle-Zemble ils parureint de nouveau. Ils

' Turver, Report of the bones of Cetacea, The Zoology of the Voyage or H. M. S. Challenger', 1880. 
ont vu une gamme d'une centaine de Beluga. Des Bulenoptera restratu ont fait leur apparition dans ees parages pendant les mois d'été 1 .

\section{MUSÉES.}

La Balcenopteru rostratu est représentée dans le plus grrand nombre de musies, soit par des squelettes complets, soit par des têtes ou des os isolés.

On commit aujourd'hui des sfuelettes des cet animal, provenant de la mer Blanche, des ile's Lofforlen, des côte's d'Islande "t du Groënland, des crites du Labrador et des Etats-Lnis, de la côte de Norvège, de la Baltique, de la mer du Nord, de la Manche et du canal st-Georyes, des côtes de Bretagne, du golfe de Gascogne, de la Méditerranée et de l'Adriatique.

La ville de Brème en a possédri le premier squelette, rui a été décrit par Alber's; apres lui, e'est le musée de Hunter, anjourd'hui le Musée du Collège royal des chirurgiens à Londres, qui est entré en possession du second exemplaire.

On en trouve des squelettes dans les villes suivantes: A Dyerdeen Ecosse), d’un animal qui a péri en 1870; à Amsterdam, d'un animal qui a échoué dins l'Y ; à Bergen (Norvège) il y en a plusieurs et le Musée en a fourni à divers autres établissements; a berlin, un squelette des Fiords de Bergen; ì Bordeaux, un crinte dinn animal qui a érhoué sur la côte de la Gironde; à Bologne, un crâne d'un animal de l'Adriatique

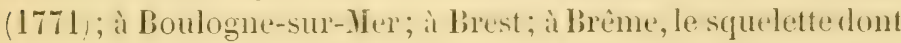
nous venons de parler; à Bruxelles, le squelette d'un animal pris dans l'Eseaut, un autre de la côte du Jutland et un troisirme du Cap-Nord; à Breslau, le squelette de 2ä pieds de l'animal inchoué en 1823 à l'île de Riügenn Baltique); à Buenos-Ayres, le squelette de l'animal capturé sur les coites de la Plata; à Cam-

1 Suurten, Verslag en Zoologische onderaokingen gedaan gedurende den tocht van "de Willem Barents o in den zomer 1578, Truschrif Tax uet AARRYGSUxdig GexootschAp, no $5,1879$. 
bridge (Musée de l'Université), la tête et une omoplate d'un animal qui a péri sur les côtes de l'île d'Islay en 1866, et un squelette complet; ì Christiania, un squelette de 16 pieds, d'un animal capturé dans le Fiord de la ville; à Copenhague; à Édimbourg, une tête de grande taille, d'un animal échoué sur les côtes d'Écosse; ì Florence, le squelette de l'individu capturé à Villefranche le 18 février 1878; à Gand, squelette d'une femelle capturée près d'Ostende le 10 juillet 1838 ; ¿̀ Giessen; à Göthemburg (Musée d'histoire naturelle); à Götingue; à Greifswald, des os, surtout un occipital, d'un animal échoué en 1810\%, au mois de mars, près de la ville; à Halle, squelette de 18 pieds d'un animal échoué en juillet 1824, ì l'est du Jutland, provenant du Insée de Meckel; au Harre, celui d'une jeune femelle capturée à Fecamp; à Hull; à IIeidelberg; ì Leide, un squelette des Fiords de Bergen; à Londres, au Musée Britannique (squelette du Groënland, de la collection de Brandt et un autre de la Tamise) ${ }^{1}$; au Musée du Collège royal des chirurgiens (squelettes de mâle adulte, de $2: 3$ pieds, des côtes de Norfolk, 1862, et d'une jeune femelle du Doggersbank, de 16 pieds, de la collection de Hunter); à Louvain, squelette du Groënland et une tête séparée, d'origgine inconnue, mais probablement de nos côtes ; à Liège, un squelette de grande taille provenant des Fiords des environs de Bergen; à Lund (Suède); à Munich; à New-York; à Oxford; à Paris, squelette des Fiords de Bergen, rapporté par Gaimard, et un autre d'un animal capturé à l'embouchure de la Seine et étudié par Gratiolet; on y conserve un très bon moulage de la jeune femelle échouée à Fecamp en 1883̈; à Rochefort, on conserve le squelette d'un mâle, qui a échoué le 26 août 1830 dans la Charente; à la Rochelle on trouve divers ossements, sans doute du mâle qui a échoué en août 1835 à Vergeroux; ì Rouen; à Stockholm, Musée royal anatomique de Carolinska Institut; à Stuttgard (squelette des côtes du Labrador); à Upsala ; à Wurzbourg, squelette envoyé par les missionnaires ;

1 From Brandt's collection, dit Gray, Catalogue..... 
à Washington, Musée national, la tête de la Balcenoptera Davidsonii, de Scammon 1.

Il existe un grand nombre de foetus de cette espèce dans diver's musées d'Europe, et qui viemnent sans doute tous de Bergen. Le Musée de cette dernière ville en possède sept.

\section{DESSINS.}

En 167ö a paru le dessin de la Balena mysticetus et d'une Balcenoptera, par Martens de Hambourg.

Sibbald, dans sa Phalainologia nov'a, a représenté des Balénoptères avec leurs évents et les plis sous la gorge.

Hans Egede en a aussi publié un dessin.

A Greifswald, on conserve une peinture faite d'après un individu échoué à l'embouchure du Weser', en mai 1699, et dont le squelette est conservé à la maison de ville de Brême; e'est cet animal que Cuvier a mentionné sous le nom de jeune. Rorqual du Nord.

John Hunter a publié une bonne figure de cette Balénoptère en 1787, d'après une femelle de 17 pieds, capturée au Doggersbank. Lacépède en a publié un dessin qui lui a été envoyé par Sir Joseph Banks.

Fred. Cuvier l'a reproduit sous le nom impropre de Rorqual Jubarte (pl. XX, fig. I) 2. Ce dessin figure aussi dans le grand atlas de Goldfuss, sous le nom de Balanoptera boops.

Scoresby en publie également un dessin 3 .

Dans le New-York Museum, de Kay, a figuré, pl. XXX, fig. 1, la Balénoptère qui a péri aux Orcades en 1808.

Dans les actes de la Société Linnéenne de Bordeaux, nous en trouvons également un, d'après un individu échoué dans le golfe de Gascogne en août 183 ö.

1 On a new species of Balanoptera. Proceed of the Californ, AсADEмy of sciencies not. IV, part. V, Ian. 1875; San Francisco, 1875, p. 269.

${ }^{2}$ Fred. Cuvier, Hist.nat. de Célacés, pl. XX, fig. 1.

s Sconesny. An account of the arctic reg., t. I, p. 485, pl. XIII, tig. 2. 
Rosenthal a ígalement publié celui d'un mâle, qui a été r'produit par Brandt et Ratzeburg dans leur Zooloyie médicule' (pl. XV, fig. 4).

Il existe encore un dessin à la Bibliothèque royale à Bruxelles, provenant de notre confrère le vieomte du Bus, d'après l'animal qui a été tué dans l'Escaut, au mois d'octobre $186 \%$, en amont d'Anvers.

Un vient de publier un dessin de l'individu pêché en mer par Le Gaulois de Fécamp.

Le professeur Hutton a publié, dans les Ammals and Magasine of natural history (ser. 4, vol. XIII, pl. XVI), le dessin d'un animal de 16 pieds $21: 2$ pouces eapturé en octobre 1873 à la Nouvelle-Zélande.

Le professeur Julius von Haast a figuré le sternum et un fanon dans les Truns. X. Z. Institute, vol. XIII, pl. III, fig. 1 et 2 .

Eschricht a figuré un fotus 1.

Fischer a publie un dessin assez grossier d'après un journal illustré qui représente l'animal échoué à St-Jean-de-Luz.

Sars a publié un dessin fait d'après un mâle de 14 1/2 pieds capturé près de Christiania en septembre 1878.

Lesson a donné une bonne représentation de cette Balénoptère mâle, échouée dans la Charente, le 26 août 1833.

Le plus beau dessin est celui que P'. Gervais a publié d'après tune planche, faite diaprès nature, en 1861, et qui est conservée dans les vélins du Muséum d'histoire naturelle de Paris.

Le capitaine Scammon a reproduit un bon dessin (pl. VII, fig. 2), sous le nom de Balanoptera Davidsonii.

C'est sans doute une Balénoptère de la même espèce, que Anderson a figurée dans Vumman expedition, pl. XLIV, et dans Inat. anil Zooloy. Reseurches, London, 1878, sous le nom de Sittang Whale?

On conserve à Paris, au Muséum, un modèle en plâtre d'après l'animal qui a échouć en février 1861 sur la côte de Bretagne.

1 Escinacut, Nord Wallhiere, pl. VI et VII. 


\section{PARASITES.}

On a trouvé divers parasites, d'abord:

Le Distoma goliath. Yan Ben. 1, qui habite le foie. Eschricht m’avait donné des exemplaires de ce Trématode, et plus tard j’en ai trouvé moi-même dans l'animal qui at péri dans l'Escaut, en novembre $186 \%$.

Ensuite le Filaria crassicauda, Creplin, qui vit dans le canal de l'urètre ou le corps caverneux.

Enfin l'Ascuris anguliuluis; Creplin l'a observé le jremier; Koren en a remis trois exemplaires à Oscar Schmidt; Krabbe pense que c'est le même Ascaris simplex qui habite le Beluya, l'Hyperoodom, le Tarval et le Lagenorhyncus albirostris 2.

Il paraît que l'intestin renferme également l'Echinorhyncus porrigens, Rud. Il a été vu déjà par plusieurs naturalistes.

On a trouvé, à diverses reprises sur la peau, des Penella Balcenopterce, sur lesquelles on a trouvé un Cirripède, le Conchoderma viryata. Cette Penella se loge surtout autour des organes sexuels.

La Balcenoptera rostrata, capturée ì Villefranche en 1878 et dont le squelette est conservé au llusée de Florence, hébergeait une Penella.

On a trouvé entre la peau et les muscles de cette même Balénoptère, comme dans certains Dauphins, des parasites enkystés, dont la nature n'est pas bien déterminée.

1 Bullel. de l'Atad. royale de Belg., ge série, $\mathrm{t}$. V, 1838.

2 Bullet. Acad.roy. des sc. danoise, .... 1878. 



\section{BALÆENOPTERA BOREALIS.}

\section{LITTÉRATURE.}

Itudolphi, Einige anatomische Bemerliungen über Balcena rostrata; Abhand. D. Königl. Akademe D. Wissenschaft. Berlin, 1820-1821.

Lesson, Histoire naturelle générale et particulière des mammiferes et des oiseaux (Cétacés). Paris, 1828.

J. F. Fray, Zoology of the Voyage of the Erebus and Terror, p. 20.

พ. Tilljeborg, Sveriges och Norges Ryygrodsdjur, Upsala, 1874.

Van Beneden et Gervais, Ostéograplice des Cétacés, p. 198, 1870.

G. Rodach, Beschreibung eines Finnwales, Arciny fun vaturgeSCHCHTE, 1875.

Xaul Gervais, Remurques sur les Balénides des mers du Japon.

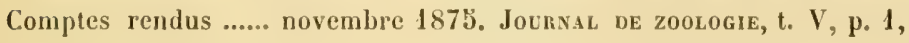
1876.

senge, a , Scelet d. Breitlöpfigen Finnwals, Plerobalana laticeps. Dautzìg, Naturf. Ges., $1876,8^{\circ}$.

P. Fischer, Sur une Balénoptère boréale, échouce à Biarritz en 1874. Conptes nexnes, $1: 76$. 
W. 'Turner, A specimen of Balcenoptera borealis, captured in the Firth of Forth. Jounn. of anat. and physiol, vol. XVI, april 1882.

Nlower, On a specimen of Rudolphi's Rorqual (Balenopt. borealis) Ialely talien on the Essex coast. Proc. zooL. Soc., nov. 20, 1885.

Guluberg, Sur rexistence de la quatrième espèce du genre Balcnoptera dans les mers septentrionales de l'Europe. Bull. ACad. Roy. D. SCIEnces de Belgique, 1884.

Guldberg, La Nature, novembre 1883.

Mfr. Er. Cockn, Notes on the Finwhate fishery of the north European Coust. The Zoologist, vol. 9, p. 107, 1886.

Eunker, Bal. borcalis al goole. Naturaliste, Londres, vol. X; ZooloGisT, vol. VIII.

abobert collett, On the External Characters of Rudolphi's Rorqual (Balanoptera borcalis). Pnoc. zooL. Soc., mareh and april 1886. 


\section{HISTORIQUE.}

Cette espèce a été longtemps confondue avec les autres: sa petite taille, comparativement à celle du Musculus et du Sibbaldii, l'a généralement fait prendre pour un animal incomplètement développé. Gràce aux recherches de II. GuIdberg, faites sur les cùles de Finmark, cette espèce est établie aujourd'hui sur des caractères aussi certains que les autres Balénoptères.

Cuvier, comparant le squelette décrit et figuré par Rudolphi avec celui de la Balénoptère de la Méditerranée, crut que ce squelette provenait d'un animal qui fréquente seul la mer du Nord, et proposa de lui donner le nom de Rorqual du Nord, par opposition au liorqual qui fréquente la Méditerranée.

Lesson, dans son histoire naturelle des Cétacés (1828), admet les trois espèces de Cuvier, et donne le nom de Balanoptera borealis au Rorqual du Nord.

C'est ce même animal que Gray proposa, dans le Voyage d'Erebus and terror, de désigner sous le nom de laticeps. Ce nom n'est pas heureux, puisque le rostre est moins large que celui des autres espèces; mais Gray avait en vue les os nasaux qui, en effet, sont plus larges que dans la Bulamoptera physalus, c'est-ì-dire du Musculus. On se figure naturellement que le mot luticeps s'applique à l'ensemble de la tête, et par conséquent au rostre.

Quelques aunées avant sa mort, V. Baer meécrivait de Dorpa! (14-26juin 18(ta): " Dans votre distributiongéographiquedes Balénoptères, je n’ai pas trouvé l'espèce qui est si commune au cap Nord et au nord de la mer Glaciale. Jai rapporté moi-même à Saint-Pétersbourg une tête de cette Balénoptère à travers la Laponie. Je vais la faire dessiner et vous envoyer le dessin. Peut-être connaîtrez-vous l'espèce d'après le dessin ».

Cette tête appartient sans aucun doute à l'espècer qui nous Tone XLI. 
occupe; malheureusement je n'ai pas recu le dessin que V. Baer me prometlait, et jo n’ai pu découvir la tête au Musée de SaintPétersbourg. Il paraît que depuis longtemps l'espace faisait défaut dans le Musée de l'Académie, et les pieces de grande dimension recevaient difficilement une place convenahle. C'est ainsi que: le superhe squelette de Balenoptera Sibbuldii, commu sous le nom de Baleine d'Ostende, dont un magnat avait fait cadeau ì l'Académie, a été relégué au Jardin zoologique, où tout est exrlusivement organisé pour l'amusement du public.

En 1876, M. Nenge a fait un travail sur le squelette d'une femelle choure le 23 aout 187 ' dans la baie de Dantzig; il la désigne sous le nom sprécifique de laticeps. M. Henge donne? une description defaillé du squelette et accompagne sil notice de quatre photographies representant fort bien le squelette.

Le professeur Zaddach de Königsblerg en a donné une description dans les drchives de Wiegmann, ave une figure de l'animal, vu de profil et vu en dessous; mais il ne partage pas lavis de Menge, qui considère cet animal comme appartenant à l'espèce Borealis.

A propos d'un animal capturé dans le Firth of Forth, le professeur Sir Turner a publié, dans les Proc. roy. Sore. d'Edimbourgr 1881-82, un mémoire comprenant la partie historique complète.

Le professeur Flower a communiqué a la Société zoologique de Londre's (20 novembre 1883 des observations sur une Balcenopleru boreulis capturée en novembre 1883 dans Crouch-River (Essex) 1. Le savant directeur du British Museum a publié un dessin du sternum de cet animal, qui présente un haut intérêt. L'animal est encore jeune. Le squelette en est destiné au British Museum.

Une nouvelle capture d'un individu de cette même espèce a été faite, en septembre 1884, sur les côtes de Lincolnshire et, en décembre de la même année, un mâle a été pris aux 0rcades.

J. Muller avait attiré l'attention sur la bifidité de la première

2 Proc. Zool. Soc, novembre 1885. 
côte du squelette de berlin, disposition à larguelle plus tard le 1) Gray avait attaché tant d'importance. ()n peut dire que dans cette espèce la première côte a une tendance particulière à devenir double; mais on ne peut pas faire un caractère spécifique de cette disposition anormale et en tout cas arcidentelle. Nous avons montré que, dans plusieurs Cétacés, la côte de la septième cervicale correspond arec l'absence de l'apophyse transwerse inferine decette vertibre. Le professen Sir Turner a publié une notice fort intéressante sur ce sujet 1 .

MIM. Chr. Aurivillius et C. Forsstrand sont allés, pendant l'été de 1877, étudier les Cútaru's ì l'étublissement de M. Siven Foyn; ils en ont rapporté beaucoup de matériaux.

Celui qui a le plus contribué à nous faire connaître cette espèce, c'est II. Guldberg, conservateur au Muser zontomique de l'Lnirersité de Christiania. M. Gulllberga a communiqué à l'Académie de Bruxelles, pendant son séjour it Liège pour y suivre les travaux du Laboratoire de zoologie, une notice, renfermant plusieurs observations précieuses qu'il a recueillies sur les côtes de Finmark, pendant les mois d'été de 1883.

M. Ciuldbery croit que c'est seulement en 1878 que l'attention a été fixée sur la Balcenoptera borealis, et que les premiers travaux sur cette espèce n’ont été publiés qu'en 1881. Nous ferons remarquer que, déjì du vivant d'Eschricht, notre attention a été fixée sur cette Balénoptère; Eschricht marait cédé un squelette ver's $18 \% 8$, squelette que jai abandonné à mon tour à M. Du Bus, pour le Muscée royal d'histoire naturelle de Belgique ${ }^{2}$.

Tandis que la péche au nord de la Norwège a principalement en vue la Balanoptera Sibbaldii, elle s'est excrée en 1883

- Cervical rits, and the so-called bicipital ribs in man, in relation 10 corresponding structures in the Cetucea. Joufiral of Axatomy and PuysioLOGY, vol. XVII.

- Nous avons fait mention, en 1868 , de ce squelette, à cause de la curieuse conformation de sa premiere cóte, Bullet.de l'Acad. royale de Belg, ge série, t. XXVI, 1868. 
presque exclusivement sur la Balanoptera borealis. La B. Sibbaldii n'y a paru qu'en fort petit nombre cette année. On a capturé presque exclusivement des Borealis, et un établissement est aujourd'hui érigé à Drammen, par la Christiania preserving $C^{\circ}$, pour la préparation de la chair de cette espèce comme aliment 1. On tire igalement partie des mandibules, dont on fait des aiguilles à tricoter.

M. Robert Collett a publié, en 1886, un travail intéressant sur les caractères extérieurs de la Balanoptera borealis, et a accomp.igné sa Notice de deux planches représentant le mâle et la femelle 2.

Après l'exposé des caractères extérieurs, R. Collett décrit sommairement les caractères distinctifs des quatre espèces de Balénoptères qui hantent le nord de l'Atlantique.

Ce travail renferme des détails fort intéressants sur les caractères distinctifs propres ì cette espèce; il fait connaître ses parasites 't ses commensaux, sa capture, ses habitudes, son âge adulte et foetal, et mème ses monstruosités.

\section{SYNONYMIE.}

Balanoptera borcalis, Lesson.

Balcena rostrala, Rudolphi.

Rorqual du Nord, Cuvier.

Sibbahlius laliceps, Gray.

Langrör ou Suaiwahl, Sorichval, Seje, Cadwhale, Sildchwal, les pêcheurs finmarkois.

\section{CARACTÈRES.}

Les individus qui échouent atteignent communément de 30 à 40 pieds; cette dernière longueur est même rarement atteinte.

- Les Chinois aiment la chair des Cétacés à fanons, et préfèrent la chair des Baleines vérilables à cclle des Balénoprtères.

2 On the External Characters of Ruclolphi's Rorqual (Balienoptera borealis). Proc. Zoor. Soc., 1886, 1). 245. 
Les fanons sont noirs arec les barbes blanches et soyeuses. La nageoire dorsale est élevée, courbée et pointue. La nageoire pectorale est noire à sa face externe, blanche à sa face interne, pointue à son extrémité.

En 1883, on a fait l'observation que les nombreux individus capturés sur les rôtes de Finmark, ont les nageoires pectorales noires des deux côtés.

Les flancs sont couverts de taches blanches (spotted with white) et le dessous blane (with a faint redtlish tinge).

Si l'on tient compte de la taille, du nombre de vertèbres ( 55 ou 56 ), de la couleur des fanons, de la forme de l'atlas et des autres cervicales, on ne peut confondre cette espèce avec aucune autre Balcinoptère. La Bulchopteru rostratu a quarantehuit vertèbres, l's fanons jaunes, et ne dépasse guère 30 pieds; la Balcenopteru musculus a plus de 40 pieds et a toujours les fanons fonrés avec des stries blanches, et près de soixante vertèbres. La Balienopter'a Sibbaldii a plus de 80 pieds, des fanons noirs et fort larges à la base.

La Butenepteru Schlegelii des îles de la Sonde, ainsi que la Balénoptère qui a été envoyée rlu Japon, ont si bien les caractères de notre Balcenoptera borealis, que MM. Flower, Turner et Paul Gervais ont été frappés tous les trois de cette ressemblance.

R. Collett domne la mesure en longueur de divers individus : les six individus qu'il a mesurés ont de 43 à $491 / 2$ pieds. Il signale même un individu de la longueur de ๖ั2 pieds.

Il donne de 33 à 37 pieds aux plus petits individus qu'il a

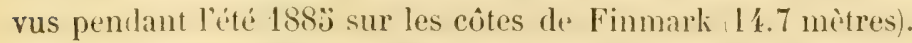

Le squelette de Berlin n'indique qu'une Iongueur totale de 31 à 32 pieds; celui de Leyde, de 32, comme le squelette du cap Nord, qui est à Bruxelles. Sars en a même vu aux lles Loffoden dont la taille varie entre 20 et 30 pieds; mais ce sont de jeunes individus 1.

- G. O. Sare, Om individuelle variationer hos Rorhvalerne. Vidensk. Selsk. Forhandlinger for 1868 . 
II. Flower a étudié le squelelte de Leyde, et trouve une longueur de 29.7 pieds, en ne tenant pas compte de l'espace intervertébral. It provient de l'animal capturé au Aloniken-Dam dans la Zuyderzée.

La longueur moyenne des individus capturés pendant le mois de juin sur les côtes de Fimmark en $188 \%$, était de 4") pieds.

Il faut conclure de cette diffírence de taille, on qu'il y' a deux races, qui prennent peut-etre chacune une direction diflérente, ou, ce qui parait plus probable, que les individus qui viennent se perdre dans la mer du Nord, sont tous jeunes.

\section{ORGANISATION.}

M. Colleft al compté dans une femelle adulte, de chaque côté, le long de la mandibule, onze bulbes pileux aree des poils de 10 millimitres, et derrière eux encore deux autres, ce qui fait vingt-six en tout.

Dans un futus de $1 \frac{1}{2}$ mètre, il existait de nombreux poils, mais très courts. Dans un autre foetus de 2.50 mètres, les poils formatrut trois rangs le long de la mandibule, la supérieure et linférinure comprenant trois poils, celle du milieu, onze, ensemble dix-sept poils de chaque côté.

A la mâchoire supérieure d'un fœetus, il n'y avait que sept poils placés sur un rangi, les deux premiers plus éloignés l'un de l'autre que les suivants.

Les fanons de l'animaldont le squelette est à Edimbourg et qui a 37 pieds de long, wereblach, strifled with yrey and white, and the huirs projecting from the lowe'r free border were greyish white (Turner).

II. Collett a publié des détails fort intéressants sur les fanons qu'il a observés sur les lieux de la capture de ces animaux.

Les deux rangée's de fanons s'unissent en avant sur la ligne médiane; Gaimard avait déjà figuré cette disposition.

Les fanons sont noirs comme ceux de Sibbaldii, mais, en dedans et en avant, ils sont d'un jaune pâle. Les barbes sont extraordinairement fines et toutes blanches. La forme des 
fanons, surtout des derniers, les rapproche de ceux des vraies Baleines par leur longueur et leur étroitesse à la base. A ne considérer que les fanons, cette espèce se trouverait entre les Baleines et les Balénoptères.

Les fanons frais ont une couleur jaunâtre (horngelb), dit Zaddach, mais dessichés, ils sont en partie d'un garis hleuatre et même noirs.

Si nous passons en revue les os du squelette, nous remarquons que cette, espèce se distingue par la largeur des os nasaux; par l'os frontal, qui n'est pas plus large à sa base qu'audessus des orbites; par les vertèbres, qui sont proportionnellement petites et au nombre de einquante-cinq ou cinquantesix; par les os en général, qui sont délicats et moins spongieux que dans les autres espèces; enfin par la première côte qui a, comme nous l'avons déjà dit, une tendance particulière à la bifidité. Cette observation, faite d'abord par J. Muller, a conduit plus tard le docteur Gray à établir des subdivisions multiples, tant parmi les Baleines que parmi les Balénoptères.

La caisse tympanique a la plus grande ressemblance avec celle de lat Buhenoptert musculus, en differant toutefois par sa forme aplatie et par ses extrémités pointues.

L'individu dont parle Zaddach a la troisième vertèbre cervicale unie à la quatrième; ce n'est guère que dans la Balcenoptera rostralu que l'on a vu jusqu'à présent ces coalescences.

La réunion de certaines vertèbres cervicales a été observée également par Ciuldbergen. P'ar cette tendance des cervicales à la soudure, comme par les finons, cette balénoptire se rapproche des vraies Baleines.

Dans un squelette du Musée de Leyde, provenant d'un animal échoué près du Moniken-Dam (Zuyderzée), et qui a été décrit par Schlegel, nous trouvons la colomme vertélnate divisée en : sept cervicales; treize dorsales; seize lombaires, et vingt caudales; en tout : cinquante-six.

Nous avons trouvé, dans l'exemplaire qui est à Bruxelles, à droite, une côte supplémentaire, attachée par des parties molles à la première côte dorsale, et à gauche, une côte 
soudée. Le squelette provient d'un jeune animal 1 capturé sur les côtes de Finmark, et dont les ligaments étaient encore tous en place au moment de son arrivée à Louvain.

La première côte est également bifide des deux eôtés dans le squelette de Leyde, provenant du Moniken-Dam, et elle s'articule avec la dernière cervicale et la première dorsale.

La Balanoptera borealis, de la côte d'Essex (188:3), a la première côte bifide des deux côtés, une partie articulée à la première dorsale, l'autre aux apophyses de la demière cervicale.

La bifidité de la première côte a été reconnue dans presque tous les individus, excepté dans celui qui a été décrit par le professeur Sir Turner (Joum. anat. et physiol., avril 1882).

Le squelette de Dantzigg, décrit par Menge et par Zaddach, montre une petite côte rudimentaire de forme triangulaire.

M. Flower a observé sur un Tursiops tursio deux côtes, chacume de :i2 millimitres de long, articulées aux apophyses transverses de la septième cervicale.

Nous comnatssons anjourdhui le sternum de qualques squelettes, ainsi que les dernieres vertibres, qui manquaient à celui dont nous avons donné la description dans notre ostéographie.

Le sternum est élargi comme celui du Musculus, mais l'atlas et le bassin ont des caractères particuliers et ressemblent beancoup à l'atlas et au Bassin de la Balénoptère de Scheveningen, qui est au Musée royal de Bruxelles.

Dans le squelette de Leyde, le sternum est plus large que long et affecte la forme d'un disque à contour irrégulier.

II. Flower a publié une figure intéressante du sternum de l'animal qui a échoué 'n 1883 Proc. de la Sociélé aooloqique de Londres). Il est petit, en partie cartilagineux, long de 7 pouces et un peu moins large que long. La première côte de ce squelette est également biceps.

Les deux os du bassin ne se ressemblent pas, dit Guldberg; celui de droite it $0^{\mathrm{m}}, 200$ de largeur; l'autre est renversé en $\mathrm{S}$ et un peu tordu; la largeur est de $0^{\mathrm{m}}, 220$.

I Bullet. de l'scad. roy. de Belg., ge série, t. XXV1, ll. I. 
D'après le professeur Struthers, le trape sölle du carpe manque dans la Bulcenoptera borealis, tandis qu'il existe dans la Balanoptera musculus 1.

On connaît quelques foetus de cette espèce. En 1885, le 28 juin, on a trouvé une femelle pleine avec un foetus de $51 / 2$ pieds anglais (Cocks).

Le marquis de Wavrin a rapporté de Varlsö, en 1883, trois foetus, un de $1^{\mathrm{m}}, 96$, un autre de $1^{\mathrm{m}}, 87$ et un troisième de $1^{\mathrm{m}}, 27$.

M. Guldberg fait mention de quatorze foetus, presque tous recueillis au mois de juillet; ils ont de $1^{\mathrm{m}}, 0 \mathrm{~b}^{2} 0$ à $3^{\mathrm{m}}, 034$.

Si l'on en juge par la taille des adultes, le Baleineau doit avoir 4 mètres en naissant.

La taille moyenne de l'adulte complètement développé est, comme nous l'avons dit plus haut, de $1 \%$ mètres environ.

\section{MOEURS.}

En général cette espèce est rare et, dans les eaux visitées par les Balénoptères, on n'en trouve qu'en très petit nombre au milieu des autres.

Dans les individus capturés sur les côtes de Finmark, on a trouvé l'estomac plein de Cirustacés, comme celui de la grande espèce.

Nous supposons qu'il y a une erreur dans l'observation de l'individu qui aurait renfermé 600 gades (Dorsche).

Il se nourrit, comme nous l'avons dit déjà, d'un Thysanopode (Euphansia inermis); en 188:3, II. Collett n'a trouvé dans l'estomac que le Copépode connu sous le nom de Calanus finmarchicus, Mull.

La chair de cette espèce est si différente de celle des autres Balénoptères, dit M. Guldberg, qu'on la conserve pour la table; il y a une pêche particulière près du cap Nordz̧d'où l'on expédie la chair sous forme de boudins. Les mandibules sont travaillées sur les lieux en aignilles ì tricoter, dit M. Cocks.

'Report, Britzsh Association, 1883, 1. 1056. 


\section{DISTRIBUTION GÉOGRAPHIQUE.}

La Seichwal n'arrive qu'en juin sur les côtes de Finmark. Le meilleur moment de la pêche de cette espece est du milieu de juin jusqu’à la première moitié de juillet (Guldberg̨).

Plusicurs baleiniexs assurent qu'habituellement elle disparaît quand les autres espèces se montrent.

Le dernier individu de la saison exceptionnelle de 1885 a été tué le 28 août. Collett dit qu'on en a vu encore le 8 septembre.

D'après les observations recueillies sur les lieux de la pêche, cette Balénoptire fait ondinarement son apparition à la fin du

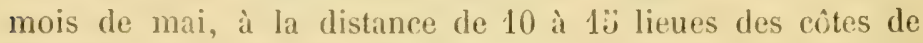
Finmarli, de nentre dans les baies que pendant le mois de juin et de juillet.

Son apparition correspond avee celle du lidulusvirens, ce qui lui a fait donner le nom de Seichval (Guldberg).

Elle arrive périodiquement au cap Nord venant de l'ouest, et, jusqu'en 188\%, elle n'avait guère dépassé le cap Nord. Cette année, les Balanoptera Sibbaldii faisaient défaut, et les Balcuoplera borealis sont arrivées en grand nombre, continuant leur route jusqu'au Varanger-Fiord.

Malmgren cite cette espèce sur le's côtes ourest du spitzberg et à la Nouvelle-Zemble; Brown ne croit pas à sa presence sur la côte ouest du Groënland.

Elle fréquente aussi la mer Blanche. L'illustre V. Baer en a enroyé un squelette à St-Pétershourg. Der won Buer mityebrachte Schüdel gehint Balzenoptera laticeps $\approx u$, merivait Brandt, le 2 janvier 1872.

M. True signale la présence de la Bulcenopteru boreulis, sous le nom de luticeps, sur les côtes des Etats-Lnis d'Amérique.

Le capitaine Scammon distingue trois Bálénoptères dans le Pacifirue', l'une sous le nom de Bulcuoptera sulfureus, c'est notre grande esperee, la Bulienuptera Sibbuldii; l'atutre la Bula- 
noptera veliferu, est bien notre Wusculus, et sous le meme nom il réunit sans doute aussi la Borealis.

La troisième espèce, à laquelle il donne le nom de Balanoptera Davilsonii est notre Rostrata.

La Balanoptera borealis est également représentée dans la mer du Japon. Le Muséum d'histoire naturelle de Paris en a reçu un squelette complet, accompagné de ses fanous.

Le Musée de Leyde en a reçu un squelette des îles de la Sonde. Il a été rapporté à une espèce nouvelle sous le nom de Balanoptera Schlegelii.

M. Flower, en étudiant les Cétacés du Musée do Leyde, a parfaitement reconnu les afînités de cette nouvelle espèce avec la Balanoptera borealis. Si ce n'était l'origine de ce squelette, je croirais avoir afiaire à une Balanoptera borealis, dit-il. Le professeur sir Turner fait remarquer que les côtes de ce squelette des Iles de la sonde correspondent ì celles de la Bulcenopteru borealis.

Paul Gervais avait reconnu également que le squelette du Muséum, provenant de la mer du Japon, ressemble beaucoup à celui de Leyde, connu sous le nom de Balanoptera Schlegelii.

Nous ne serions pas surpris de voir un des squelettes, cnvoyés de l'île Formose par le consul anglais, MI. Swinhoe, rapporté également à cette espèce.

Nous venons de voir que pendant longlenups la Buhlementeru borealis ne dépassait pas le cap Nord; c'était done à l'ouest qu'on pêchait principalement cette espèce. En 1883 c'est la Balanoptera borealis qui a été le plus abondante sur toute la côte de linmark. Les baleminiers prétendent que le Crustacé qui forme la pâture ordinaire des Sibbaldii faisait défaut cette année. En 1883 il y avait à l'Ouest einq baleiniers qui n'ont guère capturé que des Bulcenopteru borcalis; cette minte anné dix-neuf baleiniers ont capturé à l'est de ces mimes frarage's 406 Balénoptères de différentes espèces, dont so Meryaptera, 
90 Borealis, et le restant, moitié Balcenoptera Sibbaldii et moitié Balanoptera musculus. Le nombre le plus élevé par bateau était de 40 .

Guldberge pense que la Borealis est plus méridionale que les autres espèces; nous pensons, au contraire, que les Bulcenoptera musculus et rostrult sont les plus méridionales et que les deux autres sont au contraire les plus boréales. Les Balienoptera musculus el rostratı sont les seules espèces que l'on ait vues jusqu’à présent dans la Méditerranée.

Ce n'est qu'on 1878 que l'attention des baleiniers fut attirée sur cette espece, dit Guldbery, et il pense que c'est en 1881 que les premiers exemplaires furent pêchés.

Depuis 1882, on "n a pris tous le's ans à la station de Sörvär $\left(70^{\circ} 1 / 2 \mathrm{~N}^{2}\right.$-E. $)$, près de Hammerfest.

L'annér 1884, sur 3̈̈ Balénoptires de différentes espèces, on a compté, d'après un relevé, 3 , d'après un autre relevé, ¿) Borealis.

En 188̈̈ leur abondance a été telle qu'on en a capturé 750 , pendant la campagne de l'annce 1; pendant le mois de juin, on en a capturé jusqu'ì 4 par jour.

M. Guldberg nous apprend que, en 1886 , il y a eu peu de Balanephera borealis, mais plus de Balcenoptera Sibbaldii que l'anmée précédente. On a pris en tout 932 Balénoptères et Mégaptères, la plupart des Balcnoptera musculus.

On connait plusieurs individus qui sont venus échouer sur les côtes des mers d'Europe : un des premiers dont les annales fassent mention, est celui qui est venu à la cote en 1811, dans la Zuỵderée, près de Monik'n-Dam, et dont le squelette est conservé au Musée de Leyite. Van Breda en possédait le dessin.

1. 0. H. Cocks, The Finnwhale fisher! of 1895 on the North european coast. THE Zoologist, april, 1880. 
En 1816 un autre a pénétré également dans la Zuyderzée.

Le 21 février 1819, une Balénoptère a échoué sur la côte du Holstein et son squelette est conservé au Musée de Berlin.

Rudolphi a décrit ce squelette sous le titre : Einige anatomische Bemerhungen über BaLena nostrata ${ }^{1}$.

C'est cet animal que Cuvier a r'gardé ensuite comme Balénoptère du Nord et auquel il a donné le nom de Rorqual du Nord, par opposition à la Balcenoplera musculus, qu'il croyait propre à la Méditerranée. C'est le type de la Balcenoptera boreulis. Les individus qui se sont perdus n'ont guère plus de 28 ì 29 pieds de longueur, mais on compte au moins $\ddot{3} \prime$ vertèbres.

C'était sans doute une Balanoptera borealis qui est venue échouer près de liugen en 18z̈, et que Rosenthal a nommée Rostrata species major.

Le 5 arril 1826, les pècheurs de Wyk-aan-Zee trouvèrent en mer, flottant à une lieue de la còte, le corps d'une Balénoptère femelle, dont Schlegel a donné une description, accompagnée de deux planches représentant ce Cétacé, vu par ses trois faces 2 .

En 1840, un individu a été capturé à Charmouth, Dorsetshire. Etait-ce un Borealis ou un Musculus? Le squelette est perdu.

En juin 1861, d̀ l'ouest de Finmark, dans Altenfiord, un individu de 30 pieds a échoué; son squelette est au Musée de Bergen.

Entre Bidart et Biarritz (Basses-Pyrénées), un jeune mảle de 7 à 8 mètres a échoué le 29 juillet 1874. Son squelette est conservé au Musée de Bayonne 3.

Près de Bergen, dans Skogsvaag, a échoué en juillet 18833 un

1. Ném. de l'Acad. des sciences de Berlin, $18^{20 / 21 .}$

- Schlegel, Verhandeling over eenen, in het juar 1826, aan de Noordhollandsche kust gestranden vinvisch.

- Fiscier, Comptes rendus de l'Acad. des sc., décembre 1876. - Revue scientifique, janvier 1877, p. 688. 
animal, appurenmment de cette espèce, dont le squelette n'a pas été préparé 1 .

Le professeur Sars fait mention d'une Balénoptère de cette espice, ayant 30 pieds de long, et qui s'est fait capturer le 15 juin 1863 aux îles Loffoden 2 .

Dans ces dernières années, trois individus ont été capturés sur les côtes d'Angleterre; le premier dans le Firth of Forth en siptemlne 1872; son squelette est conservé au Musée anatomique de l'Université d'Edimbourg.

En novembre 1883 une autre capture est faite sur les côtes d'Essex; M. Flower en a fait part à la Société zoologique de Londres. Le squelette a été acheté pour le British II useum 3.

On went devoir échoner" an mois de septemberernier (1881) un autre individu sur les côtes de Lincolnshire, et dont. le squelette est destiné à un Musée d'Australie.

En septembre 1884, on en a vu périr un à l'embouchure du Ilumber.

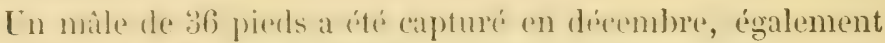
en 1884, dans la baie de Widewall Oreades'. Lr squelette a été exhibé à l'Association Britamnique de 1886, it Aberdeen 4 .

Le 23 août 1874 a péri, dans la baie de Dantzig, une Balénoptipe dont II. Henge a fait commaitre le squelefte. Du 17 au 18 août on avait ru l'animal dans la baie; les trois derniers coups de lance, donnés dans les poumons, le foie et l'estomac, l'ont achevé. M. Menge a accompagné sa notice de quatre photographies, représentant le squelette complet et les divers os séparément. La longueur est de 31 pieds 10 pouces. Il reste aux yeux de quelques naturalistes, des doutes sur la nature de cette Balénoptère.

Nous trouvons aussi quelques exemples de captures faites de l'autre côté de l'Atlantique. Dans la baie de Mob Jack (Virginie),

1 Lettre de Koren à Lilljeborg.

2 Beskrivelse ...., p. 25.

3 Proc.Zool. Soc., novembre $188 \overline{5}$.

4 Report of the british Association, 1886, p. 1050. 
en mai 1866, on a pris un M! ysticète qu'on a désigné sous le nom de Sibbuldius tuberosus, et qui parait appartenir à cette espice. Je crois que les os en sont conservés au Musée de Philadelphie. L'animal áté déerit par le profésseur Tailaferro. M. Cope croit que ce Cétacé est une Balanoptera de l'espèce qui nous occupe 1 .

\section{HUSEES.}

Le squelette de cette espèce était encore excessivement rare, il y a quelques années; il sera bientit aussi répandu que celui de la petite espèce, grâce à la péche des Rialcinoptères sur les côtes de Fimmark et de leur extrême abondance pendant l'été de 1885 .

La tête de Balanoptera, rapportée par v. Baer de la mer Blanche pour le musée de St-Pétershourg, appartient, d'après le $\mathrm{D}^{\mathrm{r}}$ Brandt, à la Balcenoptera borealis.

Le Musée de l'Université de Berlin possède depuis longtemps le seul squelette connu, provenant de l’individu échoué en 1819 sur les côtes du Holstein et décrit par' Rudolphi.

Le Musée de Leyde possède le squelette de l'animal échoué à Monilien-Dam. On fait aussi mention d'un squelette au Musée de Leyde, provenant d'un jeune individu échoué dans la Zuyderzée, vers 1816 , et qui a été acheté par un marchand d'huile d'Amsterdam. Les deux apophyses transverses de l'axis ne sont pas jointes. La première côte est biceps. Les caractìres de ce squelette ont été donnés par le professeur W. Flower 2.

Le Musée de Bergen possède deux squelettes, un des îles Loffoden, donné par le $\mathrm{D}^{\mathrm{r}}$ Daniellsen, l'autre d'un animal échoué dans le voisinage de Bergen, en juillet 1863.

Le Musée royal de Bruxelles a fait l'acquisition d'un squelette préparé au cap Nord et que Eschricht nous avait cédé.

1 Proced...... Acad., 1866, p. 8.

- Traduction du mémoire de Lilljeborg sur les Cétacés des côtes scandinaves. 
Nous avons vu, au Musée anatomique d'Édimbourg, des ossements fort intéressants d'un animal capturé dans le firth of Forth.

Le Musíe du College royal des chirurgiens à Londres renferme diver's os d'un animal de taille ordinaire, provenant des côtes d'Angleterre; parmi eux nous arons remarqué une première côte bifurquée, fort large, du côté du sternum surtout, ainsi qu'un atlas et un axis provenant d'un animal bien adulte.

Au Britısh Museum on conserve le squelette de l'animal qui a été capturé dans la rivière Crouch, en 18831.

Si je suis bien informé, le squelette de l'animal eapturé vers la même époque, également sur les côtes est d'Ingleterre, a été acheté pour un musée d'Australie.

Le Musée de Leyde possède un squelette envoyé de Java et que M. Flower n'aurait pas lésité à rattacher à l'espèce qui nous occupe, s'il ne venait pas de ces parages. Il est connu sous le nom de Balenoptera Schlegelii.

Le Muséum de Paris a reçu un squelette du Japon qui se rapproche, par tous li's caractères tirés des os, de la Bulcenoptera qu'on a nommée Schlegelii. II a de 11 à 12 mètres de longueur, et la tîte a lat même dimension que celle de notre Balonoptera borealis.

Au Musée de Christania, II. Guldberg a déposé un foetus de $1^{\mathrm{m}}, 3 \% 3 \%$, dont il a domé la description dams les Bulielins de l'Académie de Bruxelles (janv. 1884).

Le même Musée renferme un crâne, une omoplate, une troisième cervicale, les os du bassin, l'os tympanique.

II. Guldberg est en possession des trois premières cervicales d'un animal très vieux, capturé près du cap Nord (Fufjord). Ces trois vertibres sont soudées par leur bord inférieur, tout en laissant un certain espace entre le corps des vertèbres.

Nous trourons un squelette complet au Musée de Dantzig

2 List of the specimens of Cetacea in the Zoological department of the British Museum. London, 1885. 
dont le crâne est photographié. Il provient d'un animal échoué dans la Baltique.

A Bayonne on trouve le squelette de l'animal capturé à Biarritz, en juillet 187亿; il a été signalé par le $\mathbf{D}^{\mathrm{r}}$ Fischer 1.

M. Gerrard, à Londres, est, au moment de mettre cette page sous presse, en possession d'un squelette provenant d'un animal qui a échoué dans la Tamise, près de Tilbury, au mois d'octobre dernier (1887).

Le Musíe de Cherbourg est en possession de sept fanons d'un animal de cette espèce, que je croyais d'abord devoir rapporter à la Bulcmoptera Sibbaldii à cause de leur couleur noire luisante; je n'avais pas remarqué d'abord les barbes blanches. On ne connaît rien de positif sur leur origine. Peut-être ont ils été apportés par un des navires de l'État, qu'on envoie au Nord protéger la pêche d'Islande : la corvette La Recherche a été envoyée en 1834 à la recherche du brig La Lilloise.

Plusieurs Musées du Nord ont des fanons de cette espèce; nous en avons à Louvain d'un jeune animal et d'un animal adulte que nous devons à iI. Guldberg, et nous en possédions un depuis longtemps, qui nous avait été envoyé par le capitaine Jouan.

\section{DESSINS.}

Cette espèce a été rarement représentée; nous en trouvons un dessin dans Brandt, Wedicinische Zoologie (1827-1834), planche XV, figure 3, dessin qui a été exécuté par Von Mathiesen en 1819, ì Hanbourg, d'après l'animal échoué sur les côtes du Holstein; le squelette est au Musée de l'Université de Berlin. C'est la même Balénoptère qui a été décrite par Rurlolphi et que Cuvier arait confondue avec les autres espèces sous le nom de Balénoptère du Nord.

- Comptes rendus, 27 décembre 1876. et Journal de Zoologie, vol. V, p. $\{62,1876$.

TOME XLI. 
Le professeur Van Breda possédait le dessin de l'animal qui a été capturé en 1811 dans la Zuider-Zee. Nous ne savons entre les mains de qui il se trouve aujourd'hui.

Le Dr Fischer a publié un croquis, fait par MII. de Folin et E. Moreau, d'un jeune mâle qui est venu à la côte, entre Bidart et Biarritz (Basses-Pyrénées), en 1874.

Sars en a publié un dessin d'après un animal des îles Lofforlen.

Le 1) Guldberg m'a montré un dessin fait grossièrement sur place au cap Nord; il reproduit la forme du corps, mais sans la queue.

R. Collett vient de publier un beau dessin du mâle et de la femelle encore en chair.

Lindividu échoué dernièrement dans la Tamise a été photographié.

Le squelette et la tête surtout ont été dessinés plusieurs fois. Nous trouvons d'abord l'un et l'autre dans le Mémoire de Rudolphi, et Cuvier n'a fait que reproduire le dessin de la tête, dans ses Recherches sur les ossements fossiles.

Brandt et Ratzeburg ont ensuite reproduit le dessin du squelette de cette espèce, comme plus tard Pander et d'Alton.

La même tête avec la première côte ont été reproduites encore par Gray et plusieur's autres naturalistes.

Nous avons inseré un dessin du squelette dans l'0stéographie que nous avons publiée avec la collaboration de Paul Gervais, planche $\mathrm{X}$ et XI, sous le nom de Balanoptera laticeps.

\section{PARASITES.}

Les fanons, surtout ceux du milieu, se couvrent de Crustacés Copépodes en prodigieuse quantité. On trouve d'un côté des jeunes en voie de développement, et de l'autre cúté des adultes, parmi lesquels nous avons vu des femelles portant leurs oufs dans des ovisacs de forme ovale. Ce parasite ou, pour mieux dire, ce commensal, puisqu'il ne peut se nourrir aux dépens 
de son hôte, a été décrit et figuré dans les Mémoires de l'Académie de Stockholm (1879) sous, le nom de Balcenophyllus unisetus, par 11. Aurivillius. Nous l'avons trouvé en abondance sur des fanons que M. Guldberg a bien voulu nous donner.

Ce même Bulcenophyllus vit aussi sur les fanons de la Balexnoptera Sibbaldii.

M. R. Collett a trouvé dans l'intestin de tous les individus qu'il a ouverts, des milliers d'Échinorhynques, qu'il rapporte à deux espèces différentes: l'une à l'Echinorhyncus porrigens, l'autre à une espèce voisine de l'Echinorhyncus brevicollis, décrite par $\mathbf{M a l m}$ en 1867. M. Collett suppose que ce dernier Échinorhyncus est nouveau, qu'il est introduit par une pàture que la Balénoptère prend dans une autre saison et probablement dans d'autres parages. M. R. Collett lui donne le nom de Echinorhyncus ruber; il publie une bonne tigure de lanimal, de grandeur naturelle, et du rostre grossi.

M. le marquis de Wavrin a arraché de la peau d'une Balcenoptera fraîchement capturée sur la côte de Finmark, que nous supposons être la borealis, un parasite qui ne peut être qu'une Penella, d'après la description qu'il nous en a donnée. 



\section{BALENOPTERA MUSCULUS.}

\section{LITTÉRATURE.}

Ean Beneden, Notice sur une Ba'cine prise près de l'île Vlicland, el dont le squelette est monté au Jardin royal de zoologie d'Anvers. Bullet. de l'Acad. royale de Beqgique, t. XXIV, 1857.

Paul Gervais, Sur la Baleine de la Méditerranée. Bullet. de l'Acad. royale de Belgique, 2e série, t. XIV.

Dr virie, On the angatomy of Physalus antiquorum. Procend. Zool. Soc., 1865, p. 206.

v. Wower, Observations upon a Fin-whale (Physalus antiquorum, Gray) recently stranded in Pevensey Bay. Proc. Zook. Soc. of London, novembre 1865 .

C. O. Aars, Beskrivelse af en ved Lofolen indbjarget Rohrval Balœ. noptera musculus. Soershilt aftrykt af Vid-Selskabets Fonuandinger for 1865 .

Chev. Prof. v. Diorío, Il Cetaceo di S. marinella. Atti dell' Accadema PONTIFICA DEI NUOVI LINCE!, 1866.

Malm, Om elt i Zoologiska Ricksmuseum Slielell af Balœnoptera musculus frän Finmarlien. Ofversigt af liongl. Vetensk. Akad. Föriande, 1868.

w. Wlower, notes on four specimens of the common Fin-Whale (Physalus antiquorum, Gray; Balœnoptera musculus, Avct.), stranded on the south coast of England. Proc. ZooL. Soc. of Loxpos, december 1869 . 
Doñmet-adanson, Note sur le Rorqual, capturé aux cnvirons de Palavas, le 23 septcmbre 1870. Ans. Soc. D'horticulture et d'hist. nat. de z'Ilérault, Montpellier, 1870.

Van Boneden, Mímoire sur une Balćnoptère, capturée dans l'Escaut. Mém. Acad. noyale de Belgique, 1871.

Thom, Dwight, Description of the Balænoptera musculus. Nem. on the Boston Society of natur. history, vol. II, part. II, no $11,1871$.

Fried. Bracutigam, Einige Zoologisch-Zoolomische Bciträge zur Wallhierkunde. Inaug. Dissertation, Berlin, 1874.

Van Beneden, Une nouvelle Baloenoptera rostrati dans la Méditerranée. Bulletin de l'Académie, t. VIII, 1884.

Yves do agage, Histoire de la Balænoptera musculus, échouce sur la plage de Langrune. Archives de Zoologie expérnaentale, 1886. 


\section{HISTORIQUE.}

La Balcenoptera musculus est la Baléwoptère la plus commune : elle échoue sur les côtes d'Europe depuis la Laponie jusqu'au fond de la Méditerranée. Elle est aussi la plus anciennement connue: Aristote parle d'elle sous le nom de Iysticetus; il lui met dans la bouche des poils qui rappellent, dit-il, les soies du porc, ce qui ne peut s'appliquer qu'à un Cétacé à fanons.

Pline cite les paroles d'Aristote et donne à l'animal le nom de Musculus, nom que cette espèce porte généralement aujourd'hui.

Dans son Systema natura, Linné a réuni les observations faites sur ces animaux tant par les naturalistes que par les baleiniers et les voyageurs. Mais comme on ne possédait point de' squelettes dans les Musées et que les descriptions laissaient braucoup à désirer sous le rapport de l'exactitude, il n'est pas possible de dire si le naturaliste suédois a appliqué Je nom de Wusculus à l'espèce de Balénoptère qui nous occupe. D'ailleurs, si Linné s'était rendu au Groënland, il aurait eu de la peine d reconnaitre lui-même les animaux auxquels il avait donné des noms.

Un élève de Linné, Fabricius, a eu l'occasion d'étudier les Cétacés qui vivent sur les côtes de Groënland ; mais trop confiant dans la science de son maître, il a voulu se servir des noms linnéens pour désigner les animaux qu'il connaissait. Il en est résulté une certaine confusion, qui heureusement touche à son terme. On connait aujourd'hui positivement les espèces que Fabricius a dû voir sur place; mais on est loin l'être d'accord sur celles que Linné n'a connues que par des descriptions incomplètes et souvent crronées.

Ce qui montre combien nos connaissances sur la structure des Cétacés étaient peu avancées, c'est que, en 183̈, Vrolik disait, dans sa Notice sur l'anatomie d'une Balénoptère, « si on 
examine altentivement les ourruges antérieurs de IIunter, de Camper et de Cuvier, il ne peut y avoir de doute que tout notre savoir ne va guère plus loin que le squelette, le larynx, l'oil et l'oreille. ") Ce sont en effet les parties que Pierre Camper avait étudiées.

Les naturalistes sont d'accord aujourd'hui pour conserver le nom de Musculus à l'espèce de Balénoptère qui atteint une' longueur moyenne de lio pieds, qui a les fanons d'un gris verdâtre, rayés de blanc, soixante-deux vertèbres, un sternum court et large en avant, et dont le foetus à terme atteint le quart de la longueur de la mère.

C'est le Cétacé à fanons qui pénètre le plus souvent dans la Méditerranée.

Ce n'est que depuis peu que l'on connait deux exemplaires de Balanoptera rostrata, un de Bulcena biscayensis et un de Megraptera boops, qui ont pénétré accidentellement dans cette mer intéricure, tandis que la Batenoptera musculus y est asse: fréquente, au point que Cuvier la croyait propre à la Héditerranée; aussi, pour le grand naturaliste du IIuséum, c'était le Rorqual de la Méditerranée, tandis que les autres espèces n'étaient que des âges différents du Rorqual de la mer du Nord.

Nous l'avons déjà répété, toutes les Balénoptères qui pénètrent dans les mers intérieures d'Europe sont propres ì l'Atlantique, et nous pouvons même ajouter à l'Atlantiqur septentrionale.

Il est vrai, on a capturé dans ces derniers temps un .Yusculus assez jeune $\left(\dddot{o ̆}^{\mathrm{m}}\right.$. 2ல̈) pour supposer (ju'il est né dans la Méditerranée; mais quand cela serait vrai, il n’est pas démontré que ce n'est pas une mère grosse, qui, approchant des côtes pour chercher une baie propice à la parturition, a penétré accidentellement par le détroit de Gibraltar et dont le baleineau à peine né a été capturé.

On a d'ailleurs capturé dans la Méditerranée d'autres femelles accompagnées dę leur baleineau ou qui y ont mis bas.

En 1883, on en a vu une avec son jeune, au Nord-Ouest du 
cap de Creux, et le 3 septembre 1870 une femelle, presque à terme, a été capturée sur les côtes du département de l'Hérault.

On cite encore la capture d'une autre femelle accompagnée d'un Baleineau de 6 mètres.

Sur la plage de Monte-Rosso une femelle, en état de gestation, a été trouvée morte en 1878.

La taille des deux baleineaux ( $3^{\mathrm{m}}$. 25 et $6^{\mathrm{m}}$.) indique à peu près leur âge : le jeune musculus a tout au plus ว̆ mètres en venant au monde.

Schlegel a publié différents mémoires sur les animaux qui nous occupent; pour le directeur du Musée de Leyde, il n'y avait qu'une Balénoptère pour tout l'hémisphère arctique, et la Megraptera du Japon passait pour la Balénoptère antaretique; il n’y avait également pour lui qu'une seule espèce de Baleine dans chaque hémisphère.

Lorsqu'en 1834, Paul Gaimard rapporta de son voyage en Islande quelques caisses tympaniques de Balćnoptères, il ne nous fut pas difficile de reconnaitre lidentité de ces os avec ceux de la Balénoptère qui fréquente la Méditerranée; confiant dans la science de Cuvier, le Rorrual s'étend, disions-nous, jusqu'en Islande, tandis qu'en réalité cette espèce est un animal boréal qui ra se perdre quelquefois dans cette mer.

Ce n'est pas moins Cuvier qui a établi la cétologie sur sa véritable base, en nadmettant que ce que les squelettes ou les crânes connus lui avaient appris; et, de son côté, Holböll, gourerneur du Groënland, faisait des observations sur les Cétacís qui fréquentent ces côtes; il recueillait en même temps tout ce que les pêcheurs lui rapportaient, et envoyait à son ami Eschricht squelette, foetus et toutes les parties qui pouvaient ètre conservées. Il existe, en dehor's des Baleines, cinq espèces de Cétacés à fanons, disait Holböll; une est connue aujourd'hui sous le nom de Megaptera, et les quatre autres sont les Balénoptères des naturalistes actuels. Celle qui nous occupe était connue des pêcheurs sous le nom de Keporkarnak.

Eschricht a mis à profit tous ces matériaux, et on peut dire que c'est lui qui a fondé la Cétologie des naturalistes. 
Le professeur 0. (;. Sars, de son côté, a heaucoup contribué à étendre nos connaissances sur ces animaux; ayant passé plusieurs années aux Iles Loffoden, il a fait des observations inféressantes sur la Balonoptera musculus, comme sur les autres espèces, et il a comparé avec soin les divers os de leurs squelettes. Son travail spécial sur la Bálonoptera musculus est accompagné de trois planches qui reproduisent fidèlement leurs caractères extérieurs.

Le professeur de Christiania a publié ensuite, en 1868, une notice sur les variations des Balénoptéres, une autre en 1874 sur la Bulanoptera Sibbuldii, en 1878 une nouvelle sur les caractères des diverses espèces de ce genre.

Le professeur Flower a beaucoup contribué à faire connaître l'espèce qui nous occupe, en publiant diver's mémoires, parmi lesquels nous pouvons citer surtout celui qui a pour objet l'individu qui a péri dans la baie de Pevensay (Sussex) en 1863. C'était un mâle de 67 pieds de longueur, dont l'état de conservation laissait beaucoup à désirer. C'est dans ce cadavre que le directeur du British Iluseum a reconnu la véritable composition du bassin des Balénoptères. Le savant directeur du British Museum a eu l'occasion d'étudier également deux autres individus capturés, l'un à Ilargate, l'autre à Falmouth, et lé superbe squelette conservé à l'Ile de Wight (Blac Gang Chine) provenant d'un animal échoué près de Ventnor.

On a beaucoup écrit sur cette espèce, comme nous venons de le voir; on a donné des détails sur son organisation; on s'est occupé de ses caractères extérieurs, du nom qu'elle doit porter, des parages qu'elle fréquente, des lieux où des individus sont venus échouer; mais ce n'est que depuis la pêche d's Balénoptères, organisée sur une grande échelle sur les côtes de Finmark, que l'on a commencé à la reconnaître définitivement.

Parmi les auteurs qui ont le plus contribué à éclaircir l'histoire de cette espèce, nous devons citer, par ordre de date, Knox, Schlegel, Vrolik, Eschricht, Flower, Sars, et, dans ces derniers temps, Yves Delage et Guldberg. 


\title{
SYNONYMIIE.
}

Cette Balénoptère a été désignće sous un grand nombre de noms, et il y a lieu d'espérer que le nom de Balonoptera musculus lui restera.

Nous croyons que la science n'a rien a gagner à former une liste complète de tous les synonymes; il est préférable, pensonsnous, de faire un choix et de ne donner des synonymes que pour éviter la confusion.

\author{
Aysticetus, Aristote \\ Musculus, Pline. \\ Rorqual de la Méditerranée, Cuviur. \\ Physalus antiquorum, Gray. \\ Plerobalana communis, Eschricht.
}

Le Tunnolik ou Keporkarnak des Groënlandais.

Le Sildreki des Islandais.

Le Razorback des balciniers américains.

Le Finnfisch et Vinwall des baleiniers en général.

Le Rohrval des Norvégiens.

On l'appelle encore Lodde et Capelan Whale.

La Balénoptère que James Hector fait connaître sous le nom de Physalus australis, Gray, correspond parfaitement, d'après les fanons, à notre Balconoptera musculus; elle a aussi 60 pieds de long. Les vertèbres sont au nombre de 62, divisées en 7 cervicales, 15 dorsales, 15 lombaires et 25 caudales.

La Balanoptera Patachonica de Burmeister doit aussi être assimilée au Musculus.

\section{CARACTÈRES.}

La face inférieure du corps est blanche; les fanons sont d'un gris pâle verdâtre, souvent parcourus dans leur longueur par des stries blanches; les vertèbres sont au nombre de 62 et les côtes de 15 paires; le sternum est large en avant et terminé en pointe. 
La nageoire dorsale est placée au-dessus de l'anus, vers les trois quarts de la longueur du corps 1 ; elle est assez grande.

Le corps est mince et allongé comme s'il était appauvri, très comprimé au-devant de la nageoire caudale 2.

Il y a une sorte de pleuronectisme dans la Balonoptera musculus, dit M. G. Pouchet 3, en ce que la face du corps est souvent plus påle d'un côté que de l'autre. Cette observation a d'abord été faite par Sars 4; Guldberg ö croit que cette couleur blanche d'un côté se trouve tantôt à droite, tantôt à gauche.

Les fanons n'ont jamais cette couleur noire de la grande espèce, ni la couleur jaune de paille de la petite espèce; ils sont généralement verdàtres et portent des stries; ils ont moins de valeur que ceux de la Balanoptera Sibbaldii. Cependant ils n'ont pas toujours la même couleur : l'individu échoué à Palevas avait les fanons blanes, nuancés de vert, teinte qui passait même au noir sur le bord concave ou interne.

La longueur moyenne de l'animal complètement adulte est d'environ 60 pieds. M. Guldberg leur donne de 60 à 70 pieds.

Le professeur Sars pense de même que leur longueur peut atteindre jusqu'à 70 pieds, mais on peut se demander s'il n'y a pas confusion avec la $\boldsymbol{B}$. Sibbaldii.

La femelle est plus grande que le mâle. M. A. Cocks a mesuré à Vardö un mâle qui avait 61 pieds 6 pouces (anglais).

H. Cocks a vu à Vardö, au mois d'avril, un foetus de 1 pied $41 / 2$ pouces, et le 13 juin, à Eretiki, un autre de 4 pieds 6 pouces.

Mais c'est surtout ì Guldberg que nous devons des renseignements intéressants sur la taille des femelles, sur les fotus, et sur leurs mesures aux divers mois de l'été.

' Dans la Balanoptera Sibbaldii, la nageoire dorsale est placée sur les quatre cinquièmes de la longueur du corps et elle est encore plus élevée.

${ }^{2}$ Ceı animal est si mince, si allongé et si tranchant en arrière, que les marins Anglais l'ont appelé Razorback.

3 G. Pouchet, De l'asymétrie de la face chez les Cétodontes, Paris, 1880.

- Vidensk., Förhandl., 1880.

- Bullel. de l'dcad. royale de Belgique, arril 1884. 
La plupart des mères mesuraient de 63 à 68 pieds, et dépassaient donc la moyenne que nous avons donnée. Les mères, pendant la gestation, sont recherchées par les baleiniers, à cause de la graisse qu'elles ont au cceur, au mésentère et aux reins.

La mesure des foetus, trouvés dans le sein de mères capturées depuis le mois de mars jusqu'en août, et observés pendant quatre ans, présente une certaine régularité. En mars, M. Guldberg a mesuré un fœetus qui avait $126^{\mathrm{mm}}$; en avril, quatre avaient de 300 à $787^{\mathrm{mm}}$, et un $2 \mathrm{~m}, 0010$; en mai cinq foetus avaient de $32 \%$ à $975^{\mathrm{mm}}$; en juin, six avaient de 1,130 à 2,037 ; en juillet, quatre mesuraient de 1,700 à 3,100 ; à la fin de juillet, deux jumeaux atteignaient chacun 2 mètres.

On n’a pas capturé de Balénoptère à terme.

Les nouveau-nés sont estimés à 6 mètres ou 18 pieds, et peut-être faut-il réduire cette taille à $\mathbf{1 6}$ pieds.

M. Guldberg a vu également des baleineaux accompagnant leur mère: au mois d'avril, un avait de 18 à 19 pieds; un second, 18 pieds; un troisième, 21 pieds, et, au commencement de mai, il en a vu un quatrième de $\mathbf{4 0}$ pieds.

En 1883. à la fin de mai, les pêcheur's ont observé pendant deux ì trois jours, à l'Est de Finmark, une gamme de baleineaux, dans le Voranger-fiord, sous la conduite d'individus adultes : les plus jeunes avaient 20 pieds. En juin on a aperçu encore d'autres petites gammes de baleineaux, accompagnées d'individus adultes.

Le 24 juillet 1883 , une femelle, accompagnée de son baleineau, y fut encore tuée; ses mamelles contenaient plusieurs litres de lait. On n’a pas indiqué la taille du jeune.

\section{ORGANISATION.}

Il existe une poche du larynx comme dans la Balénoptère de Sibbald, et sans doute dans les diverses espèces du genre. Du 
Hamel la connaissait : on m'a assuré, dit-il 1, que les Baleines ont au-dessous du gosier un grand réservoir d'air, qui équivaut aux petites vessies à air.

Cette poche a fait commettre bien des erreurs. Ainsi F. Cuvier 2, qui n'avait sans doute jamais vu de cadavre de Balénoptère, parle d'une vessie qui, après la mort, remonte dans la bouche de l'animal et force les mâchoires à s'écarter' l'une de l'autre.

W. Vrolik s'est trouvé en présence d'un cadavre de cette espèce sur les côtes de la Hollande et a confondu cette poche avec un intestin, qui va, d'après lui, du menton jusqu'ì l'ombilic 3. Au lieu de se trouver dans la cavité abdominale, comme chez les autres mammifères, les intestins sont placés, dit-il, en dehors. On ne dira pas cependant que W. Vrolik, comme son père, nétait pas un véritable anatomiste.

Pour expliquer la présence des sillons dans les Balénoptères, Vrolik suppose qu'il sont en rapport avec la nécessité de dilatation de l'œsophage, quand l'animal avale de gros poissons, comme il a l'habitude de le faire, dit-il. - Jamais on n'a trouvé, que je sache, des restes de gros poissons dans l'estomac des Balénoptères; ce sont des poissons comme les harengs, les capclans ou les petites espèces de gades dont elles se nourrissent.

Nous arons fait dessiner cette poche et ses rapports avec le larynx dans les Bulletins de l’Académie, $3^{e}$ sér. t. II, 1881. Le professeur Sir Turner a fort bien représenté cette même poche en place dans un foetus de Balcnoptera Sibbaldii.

Le $D^{r}$ Ravin a publié quelques observations anatomiques sur les fanons d'un animal de 41 pieds de long, échoué en 1829 sur la côte du département de la Somme '.

L'étude du foetus a fait connaître que l'intestin grêle et le

1 Traile des Péches, vol. IV, p. 6.

2 Hist natur. Cétacés, $\mathrm{p} \times \mathrm{x}$.

3 Ann. Sc. nat., 1858.

+ Ann. Sc. nat., 1856, p. 266, pl. 11. 
gros intestin sont séparés l'un de l'autre, et qu'il existe un coecum peu développé.

Le professeur Struthers a publié une notice intéressante sur quelques faits d'anatomie de cette espèce : il fait connaitre avec soin la composition de la nageoire pectorale avec ses muscles fléchisseurs et extenseurs. Il décrit et figure en même temps le bassin, composé de l'os ischion et d'un rudiment de fémur avec des cartilages et des ligaments, le sternum et la manière dont il s'articule avec la première côte1

La découverte du fémur rudimentaire dans les Balénoptères à été faite en premier lieu par le professeur Flower 2 . Il parait qu'il reste toujours rudimentaire dans cette espèce.

Le squelette de l'individu échoué à Pevensey-Bay, en 1860̈, a une apophyse transverse inférieure fort courte à la sixième cervicale et une seizième côte supplémentaire.

Le professeur Struthers a fait connaître également l'existence d'une pelite côte supplémentaire dans un mâle de cette espèce.

Nous avons publié la description du squelette dans l'Ostéographie des Cétacés.

Malm donne la description du squelette et la figure des principaux os, sauf la tête, d'après un squelette de Finmark.

\section{MOEURS.}

La Balénoptère qui nous occupe est ichtyophage; au Nord ce sont surtout les Lodde's, Mallotus, c'est-à-dire, Osmerus arcticus qu'elle poursuit.

Ce poisson apparait surtout en abondance au printemps autour de l'Islande et au nord de la Norwège.

Des pêcheurs assurent en avoir trouvé jusqu'à huil cents dans un seul estomac de Balénoptère.

1 Jour Strutuers, On some points in the anatomy of a great Fin-uahle (Balanoptera musculus); on the cervical verlebre and their articulations. JoURXAL OF AXATOMY AND PHYSIOLOGY, vol VI, novembre $1871 \mathrm{et} \mathrm{vol.} \mathrm{VII,} \mathrm{1872.}$

2 Proc. Zoo!. Soc. of London, novembre 1865, p. 70.4. 
Sur les côtes d'Islande, d'Écosse et de Norwège, ce sont surtout les bancs de harengs que cette Balénoptère accompagne.

On cite encore parmi les animaux dont elle fait sa pâture : les Garles (Small cod, disent les pêcheurs) et au besoin des Schrimps.

Les Balénoptères que l'on capture sur les côtes de Finmark ont toujours leur estomac plein, tandis que les individus échoués ont ordinairement l'estomac vide. J. Murie a trouvé dans un animal échoué des débris de Méduses et des restes d'Entomostraces 1. On a trouvé aussi, dans le premier estomac, des algues qui avaient sans doute été avalées à défaut de proie.

Cette espèce souffle très haut, comme le Slätbak (Balana biscayensis), dit M. Malmgren; elle n'est pas farouche et approche des chaloupes en les côtoyant pendant des heures. C'est l'espèce la plus facile à observer.

Elle est sans doute moins farouche parce qu'on ne la poursuit guère.

D'après Guldberg, la fécondation et la mise bas ont lieu en hiver; la gestation est de dix à douze mois; le jeune accompagne sa mère jusqu'à ce qu'il ait atteint la moitié de sa taille.

\section{PÉCHE.}

Les baleiniers qui allaient jadis à la pêche de la Baleine franche dédaignèrent souvent de harponner la Musculus.

Fred. Martens aperçut en 1671, le 9 mai, une Baleine, à laquelle il aurait fait la chasse, dit-il, s'il n'avait pas aperçu sa nageoire dorsale.

R. Brown la considère encore comme sans importance à cause du peu de lard et de la difficulté de la capturer. Il se rappelle que les baleiniers trouvant un jour un cadavre flottant, qu'ils avaient pris pour un Mysticetus, l'abandonnèrent

'Proc Zool. Soc., fur. 1865, p. 211. 
aussitôt qu'ils aperçurent que c'était un Finfish; d'autres l'avaient également abandonné. Aujourd'hui on leur fait régulièrement la chasse; avant la découverte des bateaux à vapeur on ne pouvait y songer.

Lne Société anglo-américaine a organisé cette industrie dans les eaux d'Islande en 1868; elle a cessé ses opérations en 1867.

C'est au commencement de 1870 que Svend-Foyn a commencé la chasse avec des steamers et des canons chargés de bombes-lances et de harpons.

Il existe aujourd hui une péche de Balénoptères, régulièrement organisée, sur les côtes de Finmark, et parmi les espèces que l'on capture, pendant l'été, figure la Balcenoptera musculus.

La saison de cette pêche commence au mois de mai et finit au commencement d'août.

Nous avons fait remarquer plus haut que c'est la Balonoptera musculus qui arrive la première dans ces parages, et la Balcenoptera Sibbaldii, la dernière.

Le dernier animal capturé à Vardö en 1884 était une $\mathbf{V u s c u -}$ lus; le 22 août on en vit encore une à Eretiki; le 24 août on captura la dernière à $30^{\circ} 30^{\prime}$

II. A. Cocks n’a pas vu de Balénoptère dans la mer Blanche.

En partant d'Arkhangelsk le 7 septembre, il a aperçu des Balanoptera musculus à Gorodetsk Point.

Après la Balconoptera Sibbaldii c'est la Musculus qui est généralement la plus abondante sur la côte de Finmark. En

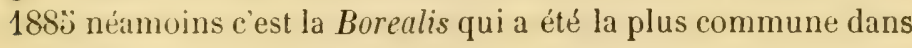
ces parages.

Les principales factoreries sont à Vardö, à Vadsü, à Büle, et à Far-Fyord.

Dans ces dix dernières années, on en a pêché sur les côtes de Finmark, en 1878, quarante; en 1879 également, quarante; en 1880 et en 1881, cinquante; (capitaine Sörensen, Guldberg.) Depuis 1881, on en a tué tous les ans plusieurs centaines. En 1886 on a capturé au moins cinq à six cents individus. Le plus grand nombre ont été pêchés aux mois d'avril et de mai. 


\section{DISTRIBUTION GÉOGRAPHIQUE.}

La Balonoptera musculus habite à l'est et à l'ouest du GroënIand, visite périodiquement l'Islande, les Loffoden, double le cap Nord, pénètre même dans la mer de Kara; elle arrive régulièrement, avec d'autres espèces, dans ces parages aux mois de mai et de juin, et passe de nouveau le cap Nord au mois d'août ou de septembre, pour se rendre de l'autre côté de l'Atlantique ou à l'ouest du Groënland.

Partout sur ce passage on a vu échouer, tantôt des mâles, tantôt des femelles, sans qu'on ait pu remarquer aucune périodicité dans leur apparition.

En partant d'Arkhangelsk, M. A. Cocks a ru des Balanoptera musculus à Gorodetsk Point, le 2 septembre, la dernière Megaptera boops le 16, la dernière Balanoptera Sibbaldii le 18, à Eretiki.

On en voit parfois se rendre au sud vers la mer du Nord, pénétrer dans le Kattegat ou bien entrer dans la Manche, longer les côtes ouest de France, les côtes d'Angleterre, d'Espagne et de Portugal, pénétrer même dans la Méditerranée et parcourir toute cette mer intérieure jusqu'au fond de l'Adriatique, sans dépasser toutefois le détroit des Dardanelles: elle ne pénètre jamais dans la mer Noire et bien rarement dans la Baltique.

Il est probable que c'est la température de l'eau et la pâture qui déterminent ces animaux à se rendre, à l'approche de l'été, de l'ouest à l'est, et, en automne, en sens opposé.

Les individus qui visitent nos parages ou qui se rendent dans la Méditerranée sont des animaux dévoyés ou blessés, ou affolés par la poursuite des harponneurs.

L'animal qui est venu se perdre, il y a quelques années, à l'entrée de l'Escaut, avait reçu dans la nageoire pectorale une balle explosible qui lui avait fracturé complétement les os de l'avant-bras.

En décembre 1870, un oflicier de garde d'une corvette de guerre (Prin: Frederic Karl) dans la mer du Nord, crut aper- 
ceroir la nuit une torpille; mais on découvrit ensuite que c'était un corps de Balénoptère commune, flottant à la surface de la mer. Le docteur Fr. Braeutigam a eu l'occasion d'étudier ce cadarre et en a fait le sujet d'une dissertation inaugurale.

Nous avons publié un plan des côtes des mers d'Europe et marqué les lieux où des individus avaient échoué. Tout ce que l'on peut en tirer, c'est que des individus de cette espèce sont allés mourir à peu près sur toutes les côtes des mers d'Europe.

La Balcenoptera musculus forme à peu près le quart des Balénoptères qui échouent dans les mers d'Europe.

Nous voyons assez souvent des individus d'une quarantaine de pieds; ce sont sans doute de jeunes Balénoptères qui ont quitté leur mère depuis peu de temps; le plus grand nombre n'atteignent pas 60 pieds; comme nous l'avons vu plus haut c'est à peu près la taille ordinaire de l'adulte.

Nous pouvons faire remarquer qu'il n'y a guère, parmi les Balénoptères échouées, des individus véritablement adultes. On avait même soupsonnć que dans cette espèce les épiphyses des vertèbres ne se soudaient pas complètement.

Nous allons faire l'énumération, par dates et par lieux, de quelques individus dont l'échouement a été signalé de manière à ne laisser aucun doute sur la détermination de l'éspèce.

Du Hamel, Bonnaterre et Lacépède parlent d'une vraie Baleine, qui fut jetée sur le littoral de la Corse, en 1620; ì en juger par la taille considérable et le nombre de barils d'huile qu'elle a fournis, le Dr Fischer pense que c'était la Balcenoptera musculus; la Balcenoptera Sibbaldii n'a jamais été vue dans la Méditerranée.

En 1624, le 28 janvier, un individu est venu à la côte de S. Narinella, et, au mois de férrier suivant, un autre à S. Severa.

En 1789, une Balénoptère de 60 pieds s'est perdue sur le littoral de la Méditerranée (sans indication de lieu).

Le crâne du Muséum de Paris, dont parle Lacépède, provient d'une femelle capturée à l'He Sainte-Marguerite, arrondissement de Cannes (Var), en mars 1797 ou 1798. 
En décembre 1827, un individu a péri sur les côtes de Corse. (Paul Gervais).

Un autre individu a péri dans la Méditerranée, le 27 septembre 1828, dans les environs de Saint-Cyprien; il a été décrit par Companyo et plus tard par Farines et Carcassonne. Il avait $13^{\mathrm{m}}, 60$. Son squelette est conservé au Musée Saint-Pierre, à Lyon. Il était mentionné d'abord sous le nom de Balanoptera aragous. Le squelette n'a que 33 vertèbres, 7 cervicales, 14 dorsales, 15 lombaires et 17 caudales. Il y en a done 9 qui manquent.

En août 1829, il échoue à l'Escale, tout près du golfe de hosas, un animal de $13^{\mathrm{m}}, 30$ en pleine putréfaction; la mâchoire inférieure a été conservée, dit M. Campanyo, qui la désigne sous le nom de Balonoptera Jubarte 1.

La même année, dans le même golfe, une Balonoptera musculus a échoué, dont le squelette est conservé au Husée de Madrid. Il n'est pas monté, m'écrit M. Mar. G. Graells.

En 1833, un individu a été capturé à Civita-Vecchia.

La mème année, un autre individu de la même espèce a été capturé, dit P. Gervais, près de Saint-Tropez (Var).

Le 21 mai 1840, une Balonoptera musculus a été capturée dans une madrague à Thone, près de Saint-Tropez, à la suite d'un coup de vent de N.-0. 2.

2. Comparyo, Histoire nalurelle du Départemenl des Pyrenées orientales, tome III, 1. 81 .

2 Nous lisons dans les Annales maritimes et coloniales, t. I, 1840: "Par le coup de vent de N.-0. du 21 mai, une Balejne s'est introduite dans les filets de l'une des madragues de Saint-Tropez (Var). Comme c'est la première fois qu'un Cétacé đe cette espèce a été pris dans notre golfe, et peut-être même dans la Méditerranée, du moins depuis plusieurs siècles, l'aflluence des curieux de la ville et des environs a été prodigieuse. Pendant quelques heures, nous avons été dans l'incertitude de saroir si c'était réellement une Baleine, attendu que quelques braves marins, habitués à ne voir que des Baleines franches, soutenaient que ça n'en élait pas une; mais, après un mûr examen, il a été reconnu que c'était la Balænoptera physalus, d'après la classification de Lacépède. » 
Au Muséum à Paris se trouve un squelette d'un animal de $14^{\mathrm{m}}$, qui a péri en 1847 sur les côtes de Saint-Vigor.

Le 10 novembre 1830 , un animal de $19^{\mathrm{m}}, 40$ a été pris à Bordigliera. Son squelette est conservé à Turin.

Une femelle de 20 mètres, accompagnée de son Baleineau, a été capturée près de Port-Vendres, en 1859, après avoir essuvé plusieurs coups de feu. La mère avait 20 mètres, le petit 6 .

Ln squelette, conservé à l'hôpital de Saint-Mandrier, à Toulon, provient d'un animal capturé en décembre 1860 (Gervais).

Gervais cite également une capture faite sur la côte de la Catalogne, en 1862 1. Nous pensons que c'est son squelette que nous avons vu a l'Lniversité de Barcelone et au sujet duquel nous n'avons pu avoir aucun renseignement sur les lieux.

Le 17 juin 1863 , une femelle de $19^{\mathrm{m}}, \mathrm{o}^{\circ} 0$ a fait son apparition au nord-ouest du cap de Creux (au delà du département des Pyrénées-Orientales, côtes d'Espagne). Elle était accompagnée de son petit et, pendant plus d'un mois, elle est restée en vue de la côte. Elle a été capturée dans la suite et le corps a été remorqué à Llanza.

En 1864, un animal a été capturé près de Cannes. Son squelette incomplet est conservé au Musée de Grenoble.

Le 4 mars 1866 , une femelle de $22^{\mathrm{m}}$ est venue à la côte de Civita-Vechia. Le squelette est conservé à Rome (chev. v. Diorio). Il a, en tout, 58 vertèbres, 15 côtes, et les. deux doigts externes ont cinq phalanges, les deux internes, six 2.

Le 23 septembre 1870, des pècheurs de Thons des environs de Palavas, département de l'Hérault (Méditerranée), relevant leurs filets par le travers du Grau de Palavas, ne furent pas peu surpris de trouver dans un d'eux un Rorqual vivant du sexe femelle de $19 \mathrm{~m}, 40$ de longueur. Amarré par la queue, il a été

1 Girvais, Mém. Acad royale de Belqique.

'Prof Vixcenzo Dionío, Il Celaceo di S. Marinella, atti dell' Agademia PONt. DeI Nuori Lixcei. Marzo, 1866. 
remorqué rivant dans le port de Cette, où l'on a pu s'assurer pendant plusieurs jours que les jets lancés par l'expiration sont formés d'air et d'eau pulvérisée. L'appareil est disposé comme un vaporisateur.

C'était une femelle qui a mis bas un foetus de $2 \mathrm{~m}, 25$. Comme les Cétacés avortent facilement par la moindre commotion, cette fausse couche a été causée évidemment par la capture 4.

L'lllustration de Paris (1570, n" 1494) fait mention d'une Balénoptère prise à Marseille, aux environs du château d'If, 26 mai 1870, et en publie un dessin.

On fait mention d'un individu capturé en décembre 1872, à Solenzana (Corse).

Un animal a échoué près d'Ajaccio, à la fin de l'année 1877 ou 1878; son squelette est conservé.

En octobre 1878, une Balanopterr musculus a été trouvée morte sur la plage de Monte-Rosso, at une petite distance entre Spezaia et Levanto; critait une femelle de 22 mètres de longueur, "n état de gestation. I I' fous est conservé à Gênes.

lans les dernires jour's de novembre 1881, une Balénoptère at été capturée dans le golfe de Cavalaire, quartier de Giova, près de st-Tropu départenent du lar, par les douaniers du poste de: Cavalaire; ils l'ont tuée en mer, it une distance de 600 à 700 mitres; une seule balle, qui a yénitré dans la tête, a suffi pour la tuer : c'est le jeune individu dont nous avons déjà parle. II n!y arait rim dims les intestins, et M. Beauregard soupconne que c'est un jenne animal qui n'avait pas plus de vingt jours d'existence. L'animal a été dépecé et tout a été envoyé au Mluséum à Paris.

MII. Pouchet et Beauregaril en ont fait connaitre les particularités de structure 2 . Yous en avions parlé dans les Bulle-

1 Nolice publiée à l'oceasion de la réunion de l'Association pour l'avancement des sciences. Douset-Anassos, Ann. de la Soc. d'horlicullure et d'hist. nat. de lllerault.

2 Poucher, Acad des sciences, séance du2 fésrier 188 ö (Revue scientifique, 7 féviges 1885̆, p.188). Compte rendu, Socjete de Biologie, t. II, janvier 1885. 
tins de l'Académie d'après des renseignements incomplets. Ce n'est pas la Balcenoptera rostrata, comme nous l'avions soupçonné, puisqu'elle a soixante-deux vertèbres, mais une jeune Balanoptera musculus, comme M. Beauregard l'a dit.

Cornalia fait comnaître onze cas d'échouements sur les côtes d'Italie, dont un dans l'Adriatique et un sur les côtes de Sardaigne.

On na guère tenu note des nombreux échouements qui ont $e u$ lieu sur la côte d'Espagne. On cite seulement une Balonopterı musculus, de grande taille, échouée au cap de Ras, entre Calère et Lianza, dont nous avons parlé plus haut.

En 1811 une Balénoptère fut jetée à la côte près de Bayonne.

Au Muséum à Paris on voit le squelette d'un jeune animal qui a péri, en 1823, devant l'embouchure de l'Adour, près de Bayonne.

On a signalé plusieurs exemples sur les côtes ouest de la France.

On en cite deux d'abord a XVII ${ }^{8}$ siecle : le premier est une Balénoptère de 47 pieds, capturée ou échouée en 1680 près de la liochelle; le second est un individu, dont on ne donne pas la taille, qui est venu ì lìle Sainte-Anne le à octobre 1682.

A Boulogne sont conservés des fragments de crâne d'un animal qui a péri, le 7 février 1812 , à l'embouchure de la Somme; d'un autre qui a péri, en 1827, à la même embouchure 1.

L'année 1812 une femelle de 26 pieds est venue se perdre également sur les côtes ouest (Souty).

Le 16 août 1829, un mâle de 41 pieds a échoué près de Cayeux. Ravin en a fait mention dans les Annales des sciences naturelles, mai 1836. Le squelette est conservé à Rouen 2. Un dessin accompagne cette notice.

- G. Poccuer, Des derniers echouemen/s de Celacés sur la côte française, Comples rendus... 2 février $188 \%$.

\& F. Ravis, Observations aralomiques sur les fanons, ANw. Sc. natur, mai $\$ 886$. 
En janvier 1842, on a vu à Berg (Pas-de-Calais) un cadavre flottant en mer (Blainville et Gervais).

En 1845, un individu a échoué sur la côte de Saint-Malo, et en 1847, un autre au Havre, dont la peau bourrée est conservée au Muséum de Paris.

A Saint-Brieux nous avons vu un squelette de mâle de 51 pieds, monté sous la direction de M. Nimier.

Un animal de 13 mètres est capturé à Saint-Vigor, à l'embouchure de la Seine, en 1847 ; la même année un jeune, mais de grande taille, échoua à Barre-des-Monts (Vendée); une Balanoptera musculus de 12 mètres de longueur a échoué près du Havre le 11 octobre 18:2, sur la plage de l'Eure. La peau a étẻ préparée et exposée au public.

Le 10 févirier 1857, les pêcheurs de Tréport ont trouvé, à l'entrée de la Manche, une Balénoptère de 60 pieds, en pleine putréfaction.

Le $D^{\text {r }}$ Fischer parle d'un mâle de 7 à 8 mìtres capturé en 1874 sur les côtes de Bayonne.

Le 6 janvier 1877, un individu de 100 mètres sans la tête est venu dans le golfe de Gascogne. Nous en faisons mention sans pouvoir assurer qu'il appartient à cette espèce.

Le 10 février 1878, les pêcheurs de Dunkerque ont remorqué un mâle de 23 mètres, dont le squelette est conservé à Lille, au Musée de l'Université catholique.

Une femelle pleine, de $20^{\mathrm{m}}, 80$ de longueur, a été trouvée morte en mer, au large de l'île de Groix (à 12 miles sud-o:rest), le 29 juillet 1879 . Le fnetus arait $1^{m}, 20$. Le squelette de la mère est au Musée de Bordeaux, celui du fotus au Muséum à Paris.

Le 21 décembre 1881, la mer a rejeté sur la côte de Porge, au nord du bassin d'Arcachon, un Cétacé de $1 \mathrm{5}^{\mathrm{m}}, 20$; le bout du rostre avec les fanons sont conservés au Muséum à Paris. Les fanons ont, parait-il, la couleur de ceux de Rostrata.

Une femelle, à l'état de cadavre, a été amenée à l'île de Sein le 19 août 1881 . Elle avait 14 mètres de longueur. Le corps flottait dans le Ras-de-Sein.

Une autre femelle, de $9^{\mathrm{m}}, 50$, a échoué à Seignasse, près du 
cap Breton (Landes', le 203 juin 1884. Le squelette est conservé au Muséum ì Paris.

Dans la nuit du 13 au 14 janvier 1883̈, un mâle est venu échouer sur la plage de Langrune. M. Yves Delage en a publié la description, dans les Archives de Lacaze-Duthiers. Le professeur de la Sorbonne a étudié avec beaucoup de soin la structure et la formation des fanons 1.

Le corps de cette Balénoptère a été vendu. D'après une ordonnance de Colbert (1681), les poissons à lard, c'est-à-dire les Cétacés, doivent, dans certains cas, ètre vendus publiquement. On s'est conformé à cette ordonnance, ce qui a empèché de commencer la dissection le jour même de l'échouement. L'animal a été mis aux enchères le 20 janvier; de la miso à prix de

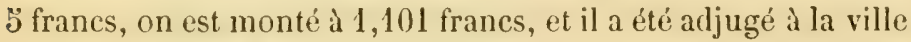
de Caen pour le Musée. Dans l'intérêt de la Caisse des gens de mer, on avait poussé jusqu'à 1,100 francs.

L'observation rigoureuse de ce règlement pourrait avoir comme conséquence, dans le cas qu'une espèce rare vînt échouer sur les côtes de France, qu'un Musée étranger pourrait venir faire une concurrence aux naturalistes français et faire prendre à l'animal le chemin de l'étranger.

L'Association française a accordé, en $18830,1,200$ francs d M. Yves Delage, pour subvenir aux frais de la reproduction héliographique des parties intéressantes de cette Balénoptère.

Le 气̋ février 1885 , une barque, montée par des pêcheurs de Gravelines, a rencontré, en face de Douvres, à une assez forte distance au large, le cadavre flottant d'une Balénoptère, qui a a été remorquée jusqu'à Ostende. C'est un mâle de Balconoptera musculus, dont le squelette est aujourd'hui au pensionnat de Melle. Les journaux de la localité l'avaient désigné sous le nom de Cachalot. Le corps en chair a été exposé quelque temps au public.

Nous pouvons citer plusieurs exemples d'échouements sur les côtes britanniques.

${ }_{1}$ Comples rendus, 6 juillet 1883. Archivos de Zoologie expérimentale. 
On en cite deux dabord dans le Firth of Forth dans le XVII ${ }^{\mathrm{e}}$ siècle, l'un, le 17 septembre 1690 , l'autre, en septembre également, en 1692; Sibbald en parle dans sa Phalainologia.

Scoresby en cite un dans le Humber, en septembre 1750, et un en 1752 , de 52 pieds, sur la côte de Berwickshire.

Walker en cite un autre, te 10 juin 1761, de 48 pieds, dans le Firth of Forth.

Sur les côtes de Cornouailles, on en cite un, le 18 juin 1797, de 70 pieds.

Le 28 octobre 1808, une femelle, de 43 pieds, a échoué dans le Firth of Forth (Pat. Neill).

En 1830, un animal de 63 pieds est venu d̀ la côte à Brighton.

Un itutre, de 38 pieds seulement, a été capturé dans le canal Saint-Georges; le corps a été remorqué à Liverpool. Son squelette "st au British .Museum, à Londres. C'est de lui que Gray avait fait le genre Benedenia.

En férriu 1810, une femelle de 11 pieds a péri sur les côtes de Yarmouth 1.

Sweeting fait mention d'un animal de $\mathbf{4 3}$ pieds, capturé sur les côtes d'Angleterre, à Charmouth, en 1840.

En 1842, un individu très adulte a été capturé à l'île de Wight; son squelette est conservé dans l'île.

Lee Is derembre 1800\%, une Balénoptère a été capturéo a Margate; son squelette est en partie à Londres et en partie à Cambridge.

On mentionne aussi une femelle de 00 pieds qui est venue à la côte aux Orcades, en mars $18 \check{6} 6$ (Heddle).

On possède à Londres un crâne d'une Balénoptère, capturée a Yarmouth, en $18 \% 7$.

Ln mâle de 60 pieds s'est perdu dans la Tamise, en 1859; le squelette est conservé à Rosherville garden (Murie).

Une Balcenoptera musculus, échouée en novembre 1863 sur la côte de Sussex, a été l'objet d'une notice fort importante

- Prar. Zool. Soc. 1810. 
dans laquelle M. Flower décrit le squelette avec l'os du bassin et le rudiment de fémur 1.

En 1869, M. Flower communique des notes a la Société de Zoologie sur quatre Balénoptères de cette espèce, échouées sur les côtes sud de l'Angleterre. Il accompagne cette noitice d'un dessin représentant un mâle de Balcenoptera musculus de 61 pieds 2.

Une Balénoptère a échoué dans Pevensey-Bay, en 18630 .

Le 20 novembre 1869, un mâle a été trouvé mort dans la Manche, près de Portsmouth. Le corps était en pleine putréfaction. Il avait 61 pieds de longueur 4 .

Le $1^{\text {er }}$ mars $187 \%$ et le 9 août suivant, on a trouvé sur les côtes de Happisburg (Norfolk), un animal mort (in the Lynn Roads), dit M. Southwell.

En avril 1880, une femelle de forte taille a péri sur les côtes d'Écosse (Baie de Forth).

Une autre a été tronvée morte en mer le 30 août 188 t (... floating in the sea off Bervic) 5 .

Sur la côte de Kerry, une Balénoptère de 63 pieds a été capturée par les gardes des côtes.

Un mâle de 50 pieds a échoué à Nairn côtes d'Écosse) en décembre 1884; son squelette a été exhibé en 1886, à l'Association britannique à Aberdeen 6 .

Une femelle de Balanoptera musculus est venue morte \& la côte, le 15 janvier 188 , à Littleton Pill, sur la Severn; le corps a été acheté par un fabricant et le squelette a été préparé pour le Musée de Bristol 7.

Sous le nom de Common Rorqual, M. Blakwill signale une

1 Proc. Zool. Soc., norembre 18650.

- Proc.Zuol Soc., décembre 1869

- Flowen, Observations upnn a Fin-Whale recenlly stranded in PevenseyBay. Proc. Zool. Soc., norembre 1863.

- Proc. Zool. Soc., décembre 1869.

- Penny illustrat. paper, ,30 aoùt 1884.

- Report of the British association, 1886, p. 10.35.

- The Zoologist, 1X, p. 107. 
jeune Balonoptera musculus, de 47 pieds, qui a péri le 3 avril 1887 à Skegness 1.

Les échouements sur les côtes de Belgique et des Pays-Bas sont assez fréquents.

On cite un échouement dans l'Escaut, le 2 juillet 1577 , d'une Balénoptère qui appartient probablement à cette espèce. On fait mention d'une autre de $\mathbf{4 2}$ pieds, qui aurait péri également dans l'Escaut, le 13 décembre 1603.

Le 14 mai 1869 , on a vu paraitre à l'entrée de l'Escaut, vers le milieu de la nuit, un énorme Cétacé qui a jeté l'épouvante dans l'esprit de tous ceux qui l'ont aperçu. Un habitant des côtes lui a envoyé une balle, puis l'animal a disparu. On l'a trouvé, peu de temps après, échoué sur la plage dite Calloot, à Borselaer, près de Flessingue 2.

Le cadavre a été vendu publiquement et un industriel de Terneuzen l'a acheté par spéculation. Il a été exposé à Terneuzen; le squelette est conservé au Musée de l'Université de Liège.

Un individu non adulte, puisqu'il n'avait que 52 pieds, échoua, en 159:3, à Zantvoort; un autre le 2 février 1598, à Bergey, entre Katwyk et Scheveningen (Clusius).

En 1601 il en échoua un à Beverwyk et un en 1629 à Noortwyk.

Le 18 novembre 1791, une femelle de 32 pieds est venue échouer à Katwyli-aan-Zee; c'est la femelle que Blumenbach a décrite et figurée.

Un squelette du Musée de Leide provient d'un animal capturé, en 1811, dans la Zuyderzee. Il a été préparé par Reinwardt.

Il y a eu encore une femelle perdue sur ces mêmes côtes, le 17 septembre 1835, et dont Vrolik a fait l'anatomie 3 .

- The Zoologist, mey, 1887.

- Van Beneden, Memoire sur une Balenoptero caplurée dans l'Escaut en 1869. MÉM. ACADÉM., 1871.

5 Vrolik, Nole sur Canatomie d'une Balénoplère, 1835, échouée près de Wyk-ian-Z ee. Ax.. Sc. Nat., 1838. 
En septembre 1836 et en septembre 1840 échouèrent deux femelles, la première de 51 pieds, la seconde de 65 pieds, toutes les deux à l'embouchure de la Meuse (Schlegel).

En décembre 1841, un mâle de 40 pieds est venu à la côte à Katwyli-aan-Zee; il a été acheté d'abord par un particulier de Scheveningen; son squelette est conservé aujourd'hui à Leyde. Il a été étudié par Schlegel et Flower.

Un autre mâle de 40 pieds est venu se perdre sur les mêmes côtes en 1844 (Schlegel).

Un måle, dont le squelette est au Jardin Zoologique d'Anvers, a échoué à l'île Vlieland, le 23 novembre $18 \% 1$.

En 1862, un individu de 50 pieds est venu à la côte à Wick.

En mars 1866, une Balénoptère de cette espèce a été capturée au Nieuven-Dam, et, en avril 1866, une autre de 47 pieds au Texel.

En novembre 1866, une femelle de 47 pieds a été trouvée flottante en mer par les pêcheurs de Scheveningen; le squelette est au Musée de Bruxelles.

Sur les côtes d'Allemagne, les échouements paraissent être moins nombreux.

Une Balanoptera musculus de 60 pieds a échoué en janvier 1721, pas loin de Brême; on l'a désignée sous le nom de Palmfisch.

En décembre 1870, un cadavre flottait dans la mer du Nord et fut pris, comme nous l'avons dit plus haut, pour une torpille.

Nous trouvons aussi quelques exemples d'échouements sur les côtes de Danemark, de Suède et Norwège.

Une femelle de 32 pieds est venue se perdre sur les côtes du Holstein en 1819.

Le 21 septembré 1841 , un mâle de 61 pieds a été capturé sur les côtes de Nord-Zélande; le squelette en est conservé à Copenhague. (Baers Bazar, 1874.)

En 1836, un individu de grande taille a échoué sur les côtes du Jutland; tout le squelette était ensablé quand on l'a décou- 
vert. Son cràne seul est retiré. Il est à Louvain, grâce à Eschricht.

Un autre individu, de 68 pieds, échoua sur les côtes de Norvège, en 1837; son squelette est à Christiania.

Le 21 septembre 1841, un mâle pourri est venu échouer dans le Kattegat.

Au printemps de $18 \not 6$, un mâle de 62 pieds est capturé à Farsund.

En novembre 1858 , un mâle de $̋ 6$ pieds périt sur la côte de Norvège; son squelette est au Musée de Bergen.

En 1872 un autre a péri sur les cûtes de Norvège; le professeur Struthers en possède l'atlas et l'axis.

En février 1867, on en a ru dans le Fiord de Christiania.

On signale la présence de Balénoptères et de Mégaptères dans la Baltique, mais s'il faut 'n croire le professeur Münter, la Balénoptère qui nous occupe ne pénètre pas, comme nous l'arons déjài dit, dans cette mer intérieure. En tous cas, clle y est rare.

Yous sommes an droit de citer un animal de ce genre qui y a péri en août $187^{\prime}$, près de Dantzig. Le professeur Zaddag en a publié une description 1. Il suppose que cet animal a pénétré dans la Baltique à la poursuite des harengs; son estomac était plein de poissons.

Il y a aussi quelques observations faites en mer sur cette espèce : le professeur Sars en a vu pendant tout l'été autour des îles Loffoden.

M. A. II. Cockis raconte que, le $10 ̈$ août, il a vu prendre à Vardö la dernière Balénoptère, un mâle de Balcenoptera musculus, de 64 pieds.

Le 22 août, il a vu plus à l'est un Musculus et un Sibbaldii; après le 2 septembre il a aperçu à Eretiki le dernier Musculus de la saison, en même temps que la Balanoptera Sibbaldii et la Megaptera boops.

' Zadoach, Beschreibung einer Finwaler, Balœnoptera musculus. ErichsoN's Авснит, $18 \%$ วั. 
En 1883, sur 406 Balénoptères capturées sur les côtes de Finmark, il y avait 90 Balcenoptera musculus.

M. Brown fait mention de cette espèce dans sa faune des mammifères du Groënland; il cite les endroits où elle stationne et indique les Gades (Cod) comme leur nourriture.

Ce n'est pas seulement sur les côtes d'Europe que l'on roit de temps en temps échouer des Balénoptères qui ont les caractères de l'espèce qui nous occupe; divers auteurs en ont signalé à la Nouvelle-Angleterre; on en voit aussi sur les côtes est et ouest, tant de l'Amérique septentrionale que de l'Amérique méridionale. Le Musće de Stuttgard en a reşu une tête de la Guyane, et le Musée de Buénos-Aỵres en possède des ossements provenant de la République Argentine.

Burmeister 1 désigne sous le nom de Balanoptera patachonica une Balénoptère des côtes du Brésil, qui correspond à notre Balconoptera musculus.

Le Muséum de Paris a reçu des squelettes incomplets de la côte de Patagonie : le sternum d'un de ces squelettes et les os naseaux sont semblables aux mêmes os de notre Musculus; mais l'omoplate est beaucoup plus allongée que celle de notre espèce commune. - C'est bien l'omoplate la plus éloignée par sa forme de celle des Mysticètes; les deux premières côtes sont toutes les deux franchement bifides. Les marins qui ont rapporté ces ossements prétendent que les Italiens ont séjourné dans ces parages avant eux et qu'ils ont emporté plusieurs os de ces squelettes 2 .

1 Dans les Proc. Zool. Soc., feb. 1833 , Burmeister publie une notice « On a New-Whale » sous le nom de Balcnoptera patachonica. II reproduit un dessin de l'atlas, axis, $4^{\mathrm{e}}$ et $6^{\mathrm{e}}$ cervicales, côte omoplate et mandibule.

\& L'expédition de la Romanche a rapporté deux squelettes de Balénoptères; l'un d'un a aimal trouvé échoué au Neuw Yeor Sound, l'autre ctait abandonné sur la plage; le premier a été préparé arec soin à hord. Parmi les ossements nous arons ru au Muséum deux rertèbres de la région lombaire, qui ne sembleut pas appartenir à ces deux squelettes. Il a été question de la mission scientifique ä Cap Horn dans la Revue Scientilique, décembre 1853. 
Nous croyons aussi reconnaître cette espèce parmi les Balénoptères signalées par le capitaine Scammon, dans l'océan Pacifique. Cet habile baleinier y a distingué trois espèces de ce genre et on ne peut pas ne pas reconnaître leur ressemblance avec les nôtres.

Le British Museum possède un squelette de l'île Formose qui a tous les aractères d'un. Musculus.

Cette espèce vit aussi dans la mer des Indes; elle y est connue sous le nom de Balanoptera Blythii; elle ne semble pas y dépasser 60 pieds.

M. Ileuglin écrit du Caire, le 30 septembre 18:1, qu’il a découvert une mandibule de Balænoptera dans la mer rouge. - Dans l'archipel de Dahlak, dit-il, se trouve ce cétacé, qui se tient dans les profondeurs et qui se nourrit de poisson. Quelquefois un poisson scie pénètre dans sa gueule et le blesse mortellement. Le cadavre est jeté sur la côte d'Abyssinie. Les habitants ne le chassent pas, mais quand il y en a qui échouent, les habitants des côtes tirent parti de leur graisse. - La mandibule a 13 pieds de longeur 1 .

Dans l'océan Austral cette même espèce porte le nom de Balonoptera antarctica.

Parmi les Balæenoptères d'Australie j'ai signalé : 1, un animal de 67 pieds, possédant 62 vertèbres, un sternum de musculus, capturé le 20 avril 1881 à $̋$ milles de Christ-Church; le squelette ressemble si complètement dans toutes ses particularités ostéologiques à celui de la Balcenoptera musculus, que ce serait extraordinaire si les deux n'appartenaient pas à la même espèe, dit Jul. von Haast 2. 2。, un autre arec 64 vertèbres, de New Brighton; ce doit être notre Sibbaldii; enfin $3^{\circ}$, une autre forme dont le squelette est à Londres et qui a tous les caractères de la Balanoptera rostrata.

- Sitzungsberichte der Math.-Nalurw. Classe d. h. Aliademis d. Wissensch. $8^{\text {tex }} 1851$. Ce Poisson-scie. Schwerd-fish, est saus doute un Orque.

z Prof. Julius vox Hast, Notes on a skeleton of Balanoplera ausiralis. Proc. Zool. Soc. 1885, p. 5̈9?. 
Une Balcenoptera du sexe mâle, de 67 pieds de long, le côté et le dos of a thush-back tint, dont la colonne vertébrale est formée de 62 vertèbres, qui a 15 côtes, le sternum semblable à l'espèce commune d'Europe, ainsi que le bassin, capturée dans les eaux de la Nouvelle-Zélande, a tous les caractères de l'espèce qui nous occupe 1 .

En résumé, au sud comme au nord de l'Atlantique, dans le Pacifique, dans l'océan Austral comme dans la mer des Indes, on signale plusieurs Balénoptères, différant entre elles de taille, et parmi lesquelles il n'est pas difficile de reconnaitre une espèce qui correspond parfaitement à notre Balcenoplera musculus.

La Balcenoptera borealis n'a été reconnue définitivement en Europe que dans ces derniers temps. Partout ailleurs, comme en Europe, il paraît que c'est aussi la plus rare des quatre, et, par conséquent, la dernière à être reconnue.

\section{MUSÉES.}

C'est l'espèce dont le squelette est le plus coinmun dans les Musées. Un des plus beaux, par son développement complet, est celui du Musée de Stockholm, provenant d'un animal capturé sur les côtes de Finmark; celui de l'île de Wight (Black Ging Chine), échoué au nord de cette île, est fort intéressant aussi par son état adulte.

Nous allons énumérer, par ordre alphabétique, les villes où l'on conserve des squelettes ou des os séparés.

A Aalesund (Norvège), on possède un atlas, un axis, une omoplate, un radius et un maxillaire inférieur d'un animal échoué sur ces côtes en 1872.

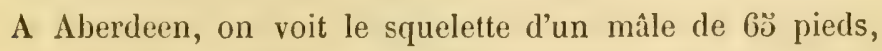
capturé, en juin 1869, près de Wick (Caithnesshire); il appar-

1 Parker, T. Seffert, Notes on a sleleton and Baleen of a Fin-Whaie (Balienoptera musculus), recently acquired by the Otago Unirersity Museum. New Zealand Journ. Sc., vol. II, no 7, p. 351.

TOME XLI. 
tient à la collection du professeur Struthers; puisqu'il a plus que la taille moyenne de l'espèce, on ne peut pas dire qu'il provient d'un jeune animal.

Le Jardin royal de Zoologie d'Anvers possède le squelette complet d'un animal échoué à l'île Vlieland (23 novembre 1831).

A Barcelone, au Musée de l'Université, on conserve le squelette de l'animal échoué le 11 juin 1863, à l'est de la ville. Au cabinet d'histoire naturelle de l'Académie des sciences et arts de la mème ville, existent les restes d'un animal très jeune, qui a péri en juillet 1833 à la plage de la Barcelonet (M. P. Graells).

A Bergen, on conserve plusieurs squelettes complets d'individus échoués dans les fiords des environs.

A Berlin, le Ilusée anatomique de l'Université possède un squelette complet des côtes de Norvège; un autre, provenant d'un mâle de 43 pieds, échoué à l'embouchure de l'Elbe en novembre 1824. Ln troisième d'une femelle trouvée morte en mer, en décembre 1870, et qui avait été prise d'abord pour une torpille.

Au Musée de Bristol se trouve le squelette d'une femelle qui a échoué dans la Savern, en janvier 1885.

A Bordeaux, on conserve le squelette d'une autre femelle de $20^{\mathrm{m}}, 80$, qui a été capturée au large de l'île de Groix, le 17 juillet 1879 . Cette femelle portait un fotus de $1^{\mathrm{m}}, 20$, qui est conservé au Muséum, à Paris.

A Bologne, on possède un squelette non monté.

A Boulogne-sur-Mer, on voit, au Musée, des ossements séparés qui ont été trouvés dans les fondations du bassin du Chatillon.

A Saint-Brieuc (France) on conserve un squelette d'un animal d'une quarantaine de pieds, qui a été capturé dans la baie de ce nom.

Le Musée royal de Bruxelles possède le squelette d'une jeune Balénoptère qui est venue à la côte, à Scheveningen, en avril 1860. 
A Buenos-Ayres, on conserve le squelette d'un animal recueilli à 10 milles de cette ville, le 3 février 1867.

Le 14 janvier 1885 , un mâle de $18^{\mathrm{m}}, 83 ٌ$ a péri sur les côtes du Calvados, à Luc-sur-Mer, dont le squelette est conservé au Musée de Caen.

A Cadix on possède un squelette monté.

Le Musée de l'Université de Cambridge renferme le squelette d'un mâle de 67 pieds, qui est venu à la côte dans la Manche, à Pevensay-Bay, en 186ə̃; la moitié d'un autre squelette, dont le restant est au Collège royal des chirurgiens, à Londres, et une tête avec ossements divers provenant de Hargate.

A Calcutta, au Musée de la Société asiatique, on conserve également un squelette qui a tous les caractères de cette espèce.

A Christ-Church, Canterbury Museum (Nouvelle-Zélande), on possède un squelette complet.

A Christiania, on voit, au Musée anatomique, des ossements de trois ou quatre individus différents. Un de ces squelettes provient d'un animal capturé dans Christiania-Fiorl, en février 1867.

Au Musée de l'Université de Copenhague, il y a un squelette complet d'un mâle de 65 pieds, qui a été capturé, le 21 septembre 1841, à Nord-Zeland.

Dantzig possèrle le squelette d'une femelle prise en 1874 près de la ville, et dont Zaddach a donné une description.

Un squelette complet avec bassin, étudié par le $\mathrm{D}^{\mathrm{r}}$ Knox, en 1831, se trouve au Musée d'Édimbourg. Il a été longtemps exposé au Jardin Zoologique. Plusieurs caisses tympaniques, recueillies dans̊ l'argile, à Stirling, sont conservées dans le même Musée par les soins du professeur Sir Turner.

A Gênes se trouve le foetus d'une femelle qui a échoué entre la Spezzia et Levanto.

A Gloucester, on conserve le squelette d'une Balénoptère, capturée près de la côte, le 15 octobre 1870 .

Gothenbourg possède un squelette complet provenant de la côte de Finmark. 
Le Musée de Grenoble renferme le crâne et quelques vertèbres d'une Balénoptère échouée près de Cannes le 14 avril 1864.

Au Musée du Havre, on voit un squelette de femelle provenant d'un animal capturé en 1885 par les pêcheurs de Fécamp.

Le Musée de l'Université de Kiel possède aussi un squelette.

Le Musée royal de Leyde possède une tête et des squelettes d'individus échoués sur les côtes des Pays-Bas.

A Leeuwaerden (Friesland) on voit, au cabinet provincial, des vertèbres et divers os recueillis dans l'alluvium.

Liège possède, au Musée de l'Université, le squelette d'un jeune animal échoué à l'embouchure de l'Escaut, en mai 1869, et un autre fort adulte des côtes de Norvège.

A Lille on conserve, au Musée de la Faculté catholique, le squelette d'un mâle de 23 mètres, trouvé en mer par les pêcheurs de Dunkerque en février 1878.

A Londres, au Collège royal des chirurgiens, on conserve la tête d'un individu qui est venu à la côte à Yarmouth en 18377; on y conserve en outre un demi-squelette dont l'autre moitié est à Cambridge.

$\mathrm{Au}$ British Museum on voit le squelette d'un jeune animal qui a péri dans le canal Saint-Georges en 1846; un autre squelette, d'un animal remorqué à Plymouth en 1831. On y conserve aussi plusieurs caisses tympaniques et des vertèbres cervicales séparées.

A Londres encore, a été exposé à Alexandra Park le /squelette d'un animal qui a été capturé en 1863 près de Falmouth ; au Rosherville garden, on possédait le squelette d'un mâle, de 60 pieds, qui a péri en mai 1859 à Gravesend.

Nous possédons au Musée de l'Université de Louvain la tête d'un animal, estimé à 70 pieds, qui a échoué en 1863 sur les côtes du Jutland et dont le restant du squelette est resté enseveli.

On conserve à Lyon, au Musée d'histoire naturelle, le sque- 
lette très adulte de la Balćnoptère qui a été capturée à SaintCyprien le 27 novembre 1828 1, et un autre, provenant d'Ajaccio, d'un animal capturé à la fin de 1877 ou au commencement de 1878; le premier a été exposé à Barcelone par M. Companyo, m'écrit M. P. Graells. Il a 60 vertèbres; c'est le premier squelette de Balénide que le sénateur de Madrid ait vu.

Le Musée de Madrid possède le squelette, non monté, d'un animal échoué le 28 juin 1832 sur la plage du port de l'Escala, Golfe de Riosas.

Au Musée de la ville de Marseille, on conserve un squelette dépareillé qui a été longtemps exposé au Jardin Zoologique. On y voit, en outre, des os provenant au moins de quatre individus différents.

A Melle, près de Gand, on possède le squelette de l'animal rencontré en face de Douvres, le ö février 188ə̃. Il a été exposé à Ostende où les pècheurs avaient conduit le corps.

A Milan on conserve au Musée un squelette qui n'est pas monté.

A Munich, on conserve au Musée la tête d'un animal capturé en 1831 dans l'Adriatique, près d'Ancone, et divers ossements séparés.

A Paris, il existe au Muséum la tête de l'animal échoué en 1797 ì l'île Sainte-IIarguerite, en face de la ville de Cannes; le squelette provenant d'un animal capturé à l'embouchure de l'Adour en 1823; un autre, d'un exemplaire échoué en 1827, à l'embouchure de la Somme; un troisième, d'un individu échoué en 1847 à l'embouchure de la Seine, et un squelette d'origine inconnue. Il s'y trouve en outre des os séparés, parmi lesquels ceux d'un animal qui a échoué le 21 décembre 1881 dans le voisinage d'Arcachon. Le squelette de la jeune femelle $\left(.^{\mathrm{m}}, 30\right)$ qui a été capturée dans la Méditerranée, dans le golfe de Cavalaire, le 28 novembre 1884 , y est également conservé.

1 Company, Histoire nalurelle du Dép. des Pyrénées-Orientales, 1. III, p. 81. 
Au Muséum à Paris, on conserve également un beau squelette des côtes de Patagonie, et une tête de Balcenoptera reçue du Japon; cette dernière a tous les caractères de notre Balénoptère ordinaire. Nous avons cru d'abord devoir la rapporter à la Balonoptera borealis, mais les os nasaux surtout indiquent une affinité plus grande avec la Balcenoptera Musculus 1.

Nous ignorons ce que le squelette de la femelle capturée aux environs de Palavas est devenu. M. Doûmet-Adanson dit qu'il est entre les mains de M. Demay.

Pise conserve le squelette d'un animal capturé sur la côte de l'île d'Elbe.

A Pontevedra (Espagne) se trouve un squelette non monté.

A Rome on conserve le squelette de la Balénoptère de S. marinella.

A Rennes on conserve une tête trouvée dans la tourbe du marais de Dol. Elle a de $\$$ à 6 mètres de longueur (Cap. Jouan).

A La Rochelle on voit la peau d'un jeune animal capturé dans le golfe de Gascogne.

Rouen possède le squelette complet d'un animal échoué au Tréport.

Le Musée de l'Académie de Saint-Pétersbourg conserve des os séparés de cette espèce, dont nous ignorons l'origine.

A Santiago (Galice) on conserve le squelette de l'animal que l'on avait pris pour une Baleine; il a un peu moins de 60 pieds de longueur. On en conserve aussi à Séville.

Le Musée de l'Académie de Stockholm possède un superbe squelette d'un mâle de ǒ8 pieds, des côtes de Finmark.

Le Carolinska Institut de Stockholm possède un autre squelette.

Le Musée de Stuttgard a reçu la tête d'un animal qui a

- Le Museum a reçu, en mème temps que la tête, une série de fanons qui ne sont pas étiquetés et que l'on croyait devoir attribuer au borealis. Ils sont verdàtres el striés de blanc. Paul Gervais a publié le dessin de la tête, vue debout, la mandibule et la caisse lympanique, daus suu Journal de Zoologie vol. V, $(18 \overline{7} 6) \mathrm{m}, 1$ et 11 . 
échoué, en 1877, sur les côtes de la Guyane hollandaise, à l'embouchure du fleuve Maroni.

A Turin, on voit au Musée le squelette monté, de 19 à 20 pieds, d'un animal échoué, en novembre 1844, sur la plage de Bordighera (côtes de Ligurie).

A Toulon, on voit le squelette d'un animal qui a échoué près de la ville en décembre 1860.

A Toulouse se trouve un beau squelette bien conservé d'un individu échoué près d'Alger en 1883.

L'Université de Valence possède le squelette monté d'un animal trouvé le 17 février à la plage de Burriana 1.

A l'île de Whigt (Black Ging Chine) on conserve le squelette monté d'un animal très adulte échoué sur les côtes de l'île.

\section{DESSINS.}

Comme cette Balénoptère a été souvent capturée, elle a été aussi très souvent figurée.

Il existe un grand nombre de planches représentant, sous le nom de Baleines, des Balénoptères et des Cachalots. Une des plus anciennes reproduit un animal qui a péri dans l'Escaut le 5 juillet 1577 ; une autre date de $1598 \%$, d'après un arimal échoué à Zandvoorde; puis une de 1598 représentant un animal échoué entre Scheveningen et Katwyk, et une de 1601, d'après un animal échoué sous Beverwyk 2.

Il y a encore deux Balénoptères figurées, une de 1629, près de Noortwyk, et une de 1791, entre Wyk-aan-Zee et Zantvoorde. On lit le nom de Vinvis sur la dernière.

Martens donne une figure de cette Balénoptère, mais il ne

- Les rensirignements sur les squelettes conservés en Espragne m'ont été donnés par le sénateur Mariano P. Graells.

- Ces diverses planches ont fait partie d'une collection appartenant à feu le Dr Huurkamp van der Vinue. Biblioth. Ichlyol. et piscaloria. Haerlem. 1873. 
reproduit pas les replis sous la gorge, ce qui est évidemment le résultat d'une négligence. Brandt et Ratzeburg ont reproduit ce dessin, pl. XV, fig. 1, sous le nom de Balanoptera physalus.

On en voit un dessin dans Lacépède ', qui a proposé le nom générique de Balcenoptera, pour les Baleines à aileron.

On voit, dans les Vélins du Muséum à Paris, le dessin d'un animal qui a échoué au Havre en 1852.

Blumenbach a fait graver le dessin d'un animal de $ّ 2$ pieds qu'il avait vu en Hollande 2. Un dessin de cette même Balénoptère a été vendu à Leeuwaerden et acheté par Van Breda. C'est probablement le dessin de l'animal qui a échoué le 18 novembre 1791, entre Katwyk-aan-Zee et Zandvoorde; une gravure, portant Vinvisch, est conservée à la Bibliothèque royale de Belgique.

Schlegel a publié dans ses Abhandlungen, pl. IX, le dessin d'un mâle de 40 pieds, capturé en 1811; d'une femelle de כ̌l pieds échouée en 1836 également sur les côtes des Pays-Bas, et un autre d'une femelle de 37 pieds échouée le 5 avril 1826 à Wyk-aan-Zee dans ses Nieuwe Verhandlungen 3 .

Nous en trouvons encore un dessin dans Zaddach 4 et dans Rosenthal 5.

Un dessin médiocre du même animal a été publié par Van Breda 6.

Ravin a publié la figure du mâle qui a échoué sur la côte du département de la Somme, en 18297.

1 LacéPÉde, Hist. nat. des Cétacés, pl. I, fig. 2, et pl. IV, fig. 2.

2 Blumerbach, Abbildungen naturhist. Gegenstaende, 8ter Heft ; Göttingen, 180 วิ.

5 Schlegel, Abhandlungen....., pl. VI, fig. 1. Nieuwe Verhandlungen nedrrl. Institut, 1818, III, pl. I et II, et 1828, III, pl. I, II et IX.

+ ZADDACH, Beschreibung eines Finwales.

5 Fr. Rosenthal, Ein. nalurh. Bemerkung. Uber die Walle, Greifswald, 1827 (Balana rostrata, Fabr. var, major).

6 Alg. Kunst en Letterkunde, 1827. L'animal est placé sur le dos.

${ }^{7}$ Ann. Sc. nutur., ge sér., I. V, 1836. 
11. le professeur Flower a publié un très bon dessin d'un mâle sous le nom de Physalus antiquorum 1.

II. Sars figure un mâle, de 40 pieds et demi de long, capturé dans les eaux de Lofoden en 18683 , et un autre mâle adulte de Varanger Fiord, pl. I 3.

II. Braeutigam publie le dessin de la femelle trouvée morte, en pleine mer du Nord, en décembre 1870 4.

II. Southwell a également reproduit un dessin de cet animal.

Le professeur Yves Delage vient de publier l'histoire de la Balcenoptera musculus échouée sur la plage de Langrune, et un atlas de $\mathbf{2 3}$ planches, dans les Aichives de zoologie expérimentale de Lacaze-Duthiers.

Il existe une photographie du squelette de Santiago, qui avait été pris pour celui d'une Baleine.

Le professeur Giglioli a publié différents dessins de Balénoptères qu'il a aperçues pendant son voyage.

Nous trouvons aussi quelques bons dessins de Balénoptères dans l'intéressant livre du capitaine Scammon, sur les animaux marins de la côte Nord-Ouest de l'Amérique du Nord.

Jul. von Haast a reproduit le dessin du sternum et du bassin de la Balénoptère qui a été capturée à peu de distance de Christ-Church (nouvelle Zélande.)

\section{COMMENSAUX ET PARASITES.}

Le professeur Sars et Sophus Hallas ont trouvé des Penella Balcenopterce, enchassées par la tête, dans l'épaisseur de la peau ;

$\&$ Flower, Notes on fuur specimens of the common Fin-Whale (Physalus antiquorum), Proc. Zool. Soc., pl. XLVII, 1869.

- SARs, Beskrivelse af en ved Lofoten indb. Rohrval Balcnoptera musculus. Aftrykt af Vid-Selskabets Forhandl, for 1885.

3 Fortsaste (1880), pl. I.

+ Fr. Braeutigam, Ein. Zool.-Zoot Beitrïge zur Walthierkunde. Inavgural-Dissertation, 29 juli, 1874. 
ils les ont observées aux îles Lofoden et sur les côtes d'Islande. Sars a vu des Penella d'un pied, avec la partie antérieure du corps plongée dans la couche graisseuse; sur la partie extérieure du corps vivait un cirripède, Cineras vittata.

Le $\mathrm{D}^{\mathrm{r}}$ Murie signale des Echinorhynchus en abondance dans l'intestin. Il reste à déterminer s'ils se rapportent à l'Echinorhynchus porrigens de Rudolphi, ou à l'espèce de la Balcenoptera Sibaldii, que Malm a désignée sous le nom spécifique de brevicollis. 


\title{
BALÆENOPTERA SIBBALDII.
}

\author{
LITTÉRATURE.
}

Sibbald, Phalainologia nova, Edinburgh, 1692.

mosenthal und Mornschuch, Epist, de Balcenopteris quib. Gryphiæ, 1825.

Van wreala, Eenige byzonderheden omtrent den Walvisch, die den 5jen november 1827 by Oostende gestrand is. Algem. Kunst-en-LetterBODE, 1827, no 48.

Du Uar, Ostíographic de la Baleine échouée à l'est du port d'Ostende, le 4 novembre 1827. Bruxelles, 1828.

P.-L. Vanderlinden, Notice sur un squelette de Balénoptère, cxposé à Bruxelles cn juin et juillel 1828. Bruxelles, 1828.

Ch. Morren, Over de Balcenoptera rostrata van Fabricius on beoordeeling des werken, welke over cen dier dezer soort, den 4 november, ter oosten van de haven van Oostende gestrandt, uitgegeven zyn. BYDRAGEN TOT NATUURKUNDIGE WeTENSCHAPPEN, 1829, pp. 52-84.

P.-X. Vanderlinden, Quelques observations en réponse $\dot{a}$ un article de M. C' Morren, sur la Balénoptère échouéc près d'Ostende... Messager des sciences et des arts, publiẻ à Gand.

Ternaert, Sur la Baleine échouée près d'Ostende, 1827. Paris, 1829, $8^{\circ}, 62$ pages.

Dr. Hobert Knox, Anatomy of the Rorqual, Proc. Roy. Soc., Edin., $\operatorname{march} 18,1855$. 
Gray, Proc. Zoor. Soc., 1847.

Nlower, On Physalus Sibbaldii, Proc. ZooL. Soc., W., 1865, p. 470.

A. VIaIm, Nagra Blad om Hvaldjur $i$ allmänhet, och Balcenoptera Carolince isynnerhet. Göteborg, 1866.

A. W. MaIm, Monograplice illustrée de la Balénoplère tronvée le 29 oclobre 1863 sur la côte occidentale de Suède. Stockholm, 1867.

J. Teinharat, Nogle Bemärlininger om Isloendernes steypircydr; aftryk af videnskab. Meddels. F. D. Natunu. Foren, 1867, nos 8-11. Kiobenhaven, 1868.

Cophus IIallas, Optegnelser om nogle pac et hvalfangst Tog.... Vidensk. medd, fra den naturist. Foren for, 1867.

w. II. Flower, On the probable idently of the Fin-Whales, described as Balcenoptera Carolince. Proc. Zoot. Soc., mars 1868.

w. Turner, $A$ n account of the great Finner-whale (Balænoptera Sibbaldii) Stranded at longniddry. Transact. of the noy. Soc. of EdinBURGH, vol. XSVI, 1870.

G. O. kars, Om Blaahvalen, Christian. Vid-Selskabs Forhandlinger for 1874 .

P.-J. Van שeneden, Notice sur la grande Balénoptère du Nord, d'après les notes tirées du Journal de voyage du docteur Otto Finsch, de Brême. Bullet. Acad. royale de Belgique, 2 érie, t. XXXIX, juin 1875.

Julius vunter, Ueber Zwei, im 19 Jahrhunderte bei Greifswald zur Section gelangte männliche Individuen von Balanoptera Sibbaldii Van Ben, Greifswald, 1877.

Sars, Bidrag til en noiere characteristik af vore Bardeltvaler. VidSelsk., Forh, 1878.

P.-J. Van Beneden, Une page de l'histoire d'une Baleine, .... Bulletin ...., 5e sér., t. II, 1881. 
Tycho Tullberg, Bau und Entwickelung der Barten bei Balcenoptera Sibbaldii, Nov. act. Reg. Soc. Scientiar., Upsaliensis, Ser. tert. vol. XI, 1883.

Burmeister, Atlas .... de la Républiqve argentine.... in fol. BuenosAyres, 1885.

Prof. Mabius, Ueber einen bei Sylt gestrandeten Blauwal (Balænoptera Sibbaldii). Scurift. D. Naturwiss. Verens für Schleswig-HolSTEIN, Bd, VI, Kiel, 1883.

c. Smets, Notes sur la tête d'un foetus de Bal. Sibbaldii. Ann. Soc. Scientif. de Bruxelles, 9e année 1885.

mor Gustav Guldberg, Zur Biologie der Nordatlantischen Finualarten. Zoologischer Jahrü̈cher, novembre, 1886.

Fobert Gray, Notes on a Voyage to the Greenland Seas in 1886. The Zoologist, april, 1887. 


\section{HISTORIQUE.}

Le terme générique de Balénoptère est de Lacépède. Il est généralement adopté aujourd'hui pour les Baleines qui ont une nageoire sur le dos. Les baleiniers les désignent sous le nom de Finnfish.

Le mot spécifique de Sibbaldii, proposé par Gray pour la plus grande espèce animale, c'est-d̀-dire pour la Balénoptère, qui dépasse parfois 80 pieds en longueur, a été introduit par le savant directeur du British Nuseum, en 1847. Il est à regretter qu'il n'ait pas toujours été aussi heureux en proposant des noms nouveaux.

Gray a proposé ce terme spécifique de Sibbaldii en voyant le jeune squelette de 47 pieds de long qui est conservé au Musée de la Société philosophique de Hull; on doit lui rendre cette justice que, s'il n'a pas connu les caractères distinctifs ou spécifiques de l'animal, il a reconnu au moins ses affinités avec les autres Balénoptères, que Sibbald avait décrites à la fin du XVII' siècle.

En effet, en 1692, sir R. Sibbald avait fait connaitre deux Balénoptères échouées sur les côtes d'Écosse; l'une, un mâle, de 18 pieds de long, l'autre, un animal de 46 pieds de long; tous les deux s'étaient perdus dans la même baie de Forth. La première se rapporte sans aucun doute à l'espèce que nous désignons sous le nom de $B$. Sibbaldii.

Sous le nom de Physalus, Pallas avait déjà parlé de Balénoptères de 84 pieds, qu'il avait observées dans les mers du Nord en 1740. Il n'est pas douteux que la Balcena physalus de Pallas, qualifiée de vulgatissima species, in mare boreo et oceano orientali, ne soit l'espèce qui nous occupe, car c'est la seule qui atteigne cette taille '. Du reste, les caractères des fanons indiqués par l'illustre voyageur, suffiraient pour la reconnaitre.

1 Pallas, Zool. Ross. As., p. 290. 
Le même naturaliste fait mention également d'un squelette de Fimfish, c'est-à-dire de Baleine à nageoire dorsale, rapporté par Petrus Kargin au Musée de St-Pétersbourg. L'animal a 84 pieds anglais de longueur, dit-il, des fanons d'un noir bleuâtre; mais il accorde à ceux-ci une longueur de 10 à 12 pieds. Il y a évidemment une erreur à ce sujet, puisqu'il n'y a pas de Baleine ì nageoire dorsale avec des fanons de plus de 4 ou 50 pieds. Cette Balénoptère de la mer glaciale, à en juger par la taille ainsi que par la couleur des fanons, est, sans aucun doute, de la même espèce; les Islandais la connaissent sous le nom de Steypireydr.

Mais on a cru longtemps, avec Cuvier, que toutes ces différences de taille devaient être attribuées à des différences d'âge. Le célébre naturaliste du Muséum admettait un Rorqual de la

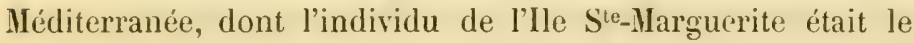
type, et un Rorqual de la mer du Nord, dont le squelette de Berlin était l'autre type.

Le 4 novembre 1827, les pêcheur's d'Ostende rencontrèrent en mer le corps flottant d'une grande Balénoptère dont les fanons étaient enlevés. Ils remorquèrent l'animal jusque dans le port d'Ostende. Un particulier en fit l'acquisition; l'animal fût dépecé, le squelette monté et exhibé à Ostende, à Bruxelles, à Paris, à Londres, à St-Pétersbourg, ensuite dans d'autres villes de l'Europe. Il échoua à la fin à Kazan; un magnat l'acheta et en fit don à l'Académie des sciences de St-Péter'sbourg. Le Musée de l'Académie n'ayant point de place, l'a fait mettre au Jardin Zoologique où il se trouve encore.

Au milieu des festivités, célébrant l'heureux échouement de la Balénoptère, à Ostende, au milieu de l'affluence de milliers de visiteurs venant contempler le squelette du géant des mers, la science ne fut pas négligée: Dubar, chirurgien à Ostende, publia une description du squelette et figura les principaux ossements.

Divers naturalistes cherchèrent à déterminer l'animal : Van Breda, Vanderlinden, Morren, s'en occupèrent et Vanderlinden émit l'avis que cet animal n'était pas connu; il proposa de 
le nommer la Balénoptère d'Ostende. Vanderlinden avait raison.

Si l'on suivait les errements généralement adoptés aujourd'hui, cette espèce devrait porter le nom de Balénoptère d'Ostende, ce nom ayant la priorité sur celui de Gray, qui n'a été proposé qu'en 1847.

Si l'échouement d'Ostende a fait progresser l'ostéologie des Balénoptères, il a peu servi à la comnaissance des espèces, faute surtout de matériaux de comparaison dans les Iusées.

Aussi en 1841 Schlegel ne recomnaissait parmi les Finntische 1 que la Balcena sulcutu arctica et la Balcena sulcata antarctica.

En 1847 Gray fit part, à la Socicté Zoologique de Londres, de ses observations sur un squelette de Balénoptère qu'il avait olsscrvé au Musée de la Société philosophique de Hull; ce squelette provenait d'un jeune animal, rui n'avait pas moins de 50 pieds de longueur, et qui ne se rapportait pas à une espèce connue. Il provenait d'un animal échoué dans le Humber, Yorkshire. Gray proposa de le désigner sous le nom spécifique de Sibbuldii, pour rappeler le nom du naturaliste Écossais qui avait écrit sur ces animaux à la fin du XVIIe siècle.

Plusieur's cétologistes remarquaient, à cette époque, que certains os de Balénoptères dépassaient considérablement les dimensions des mêmes os dans l'espèce ordinaire Balanoptera musculus). Le Musée de Copenhague possédait une nageoire pectorale des côtes du Groënland ì dimensions extraordinaires. De mon côté j’avais observé quelques os extraordinaires par leur dimension, et ces ossements provenaient-ils d'une espèce nouvelle ou appartenaient-ils à une Balcenoptera musculus géante? Les cétologistes étaient dans le doute. Pendant un voyage que je fis à Copenhague en 1856 , il nous parut, à Eschricht comme ì moi, que ces os ne pouvaient provenir que d'une espèce distincte, de grande taille, et qu'il fallait la nommer Balanoptera gigas.

1 Abhandlungen..., Leyden, 1841. 
Dans le courant de l'année 1825, une Balénoptère pénétra dans la Baltique et fut capturée le כٌ avril sur la côte occidentale de l'île de Rügen; elle avait 44 pieds 10 pouces de longueur. Rosenthal et Hornschuch nous en ont laissé une description 1.

En 1862, une seconde Balénoptère, morte dans le Kattegat, fût entraînée par le courant dans la Baltique jusqu'à la côte ouest de l'île de Rügen, où les pêcheurs la découvrirent à la fin du mois de juillet.

Le professeur Jul. Muntër eut l'occasion d'étudier les squelettes de ces animaux à Greifswald. Il était d'accord avec Sigism. Schulze sur leur identité spécifique, mais, ne sachant à quelle espèce il fallait les rapporter, il fit une visite au Musée de Leyde; il se persuada bientôt que cette Balénoptère était nouvelle poux la science et proposa, ì la réunion des naturalistes allemands, en 1863, à Stettin, de la désigner sous le nom de Balcenoptera gryphus 2. Plus tard, le professeur de Greifswald visita les musées de Hull et d'Edimbourg et il s'assura que sa Balanoptera gryphus ne différait pas de l'espèce' que Gray avait désignée sous le nom de Bulenoptera Sibbaldii.

Ces Balenoptères ne furent décrites, sous leur vrai nom, par Jul. Münter, qu’en 18773.

Il est à remarquer que la Balénoptère de Hull avait à peu près le même àge que les deux individus de la Baltique, à en juger par leur taille, qui ne dépassait pas cinquante pieds.

En 1863, le professeur Flower, visitant la collection de Lidt de Jeude, ì Ctrecht, fût frappé de la largeur du rostre d'une tête de Balénoptère, provenant d'un animal capturé sur les cotes de Hollande; il n'hésita pas de la rapporter à une espèce nouvelle pour la science et il proposa de la désigner

1 Rosmantha el Hornschuch, Eqistola de Balaropteris quibusdam, Gryi hix, 1893.

* Tageblall der 58 Versammlung deulscher Nalurforscher und Aerlzle, in Steltin, 1803.

3. Jel. Müxter, Uber Zwei in 19 Jahrhunderle bei Greifswr!dm munliche Individuen von Balenoptera Sibbaldii, Greilswald, 1877.

Tоме XLI. 
sous le nom de Balanoptera latirostris, nom qui aurait dủ lui rester 1.

Peu de temps après, M. Flower reconnut que la tête d'Utrecht appartenait à la même espèce que le squelette de Hull, et il abandonna le nom qu'il avait proposé 2 .

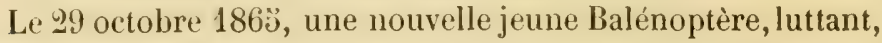
pour se sauver, sur les côtes de Suède, près de Göteborg, fut heureusement capturée; elle a été l'objet d'une monographie illustrée, avec dix-huit planches, contenant vingt-neuf photographies, deux planches lithographiées et trois gravures en bois dans le texte. L'auteur, A.-W. Malm, a décrit cet animal dans tous ses détails et, comme il lui paraissait nouveau pour la science, il a proposé de le nommer Balanoptera Carolina. Le terme spécifique était le nom de madame Malm.

L'année suivante, II. Flower publia une note : On the probable identity of the Fin-Whales described as Buldenoptera Caroline. II. Flower avait parfaitement reconnu l'espèce :3.

En 1868, on commence à faire la pêche aux Balénoptères dans les eaux d'Islande.

Hallas, médecin à bord d'un des baleiniers, qui y a fait la chasse à ces Cétacés durant deux ou trois ans, est un des premiers qui ait bien fait connaître l'espèce que l'on chasse; il a envoyé une tête et des ossements à Copenhague, et Reinhardt a publié les documents et la description des principaux os du squelette. Il a figuré la tête, l'atlas, les os nasaux et l'os hyoïde 4 .

A la même époque, le Musée de Leyde reçut du capitaine Bottemanne plusieurs pièces importantes du squelette d'un individu de cette espèce, capturé dans les mêmes eaux, parmi

1 Notes on the Skeletons of Whales in the principal Museum of Holland and Belgium, Рнос. Zool. Soc., november, 1864 p. 410.

z On Physalus Sibbaldii, Proc. Zooz. Soc., june, 1865, p. 470.

s Proc. Zool. Soc. of London March, 1866.

- J. Reiniandr, Nogle Bemarkninger om Islandernes Steypireydr., Copenharue, 1868. Videnskab. Meddels. Fra den naturhistoristie Forening for 1867. 
lesquelles se trouvent un sternum, des vertèbres cervicales et d'autres ossements. Bottemanne dit que les Islandais connaissent cet animal sous le nom de Steypireydr, et qu'il n'atteint pas moins de 80 pieds de longueur; sa coloration est foncée, dit-il.

Le 3 novembre 1869, une Balénoptère femelle pleine, de l'espèce qui nous occupe, vint échouer dans la baie de Forth; elle fut l'objet d'un travail fort intéressant de la part de Sir W. Turner, professeur d'anatomie à l'Université d'Édimbourg. Le foetus mâle avait 19 pieds 6 pouces. II s'étendait dans le corps de la mère en avant jusque tout près des condyles du maxillaire inférieur. Pour avoir une idée de l'organisation de la Balénoptère qui nous occupe, il faut recourir à ce beau mémoire.

Le professeur Sar's, fils, pendant un séjour de plusieurs années aux îles Lofoten, a fait de son côté des observations intéressantes sur les différentes espèces de Balénoptères, et il distingue parfaitement la grande espèce. En 1874, il publia également une notice sur cette même Balénoptère, d'après des matériaux recueillis sur les côtes de Finmark. Cette notice est accompagnée d'un bon dessin, représentant un foetus, d'un pied et quelques pouces de longueur.

En 1873 , le Dr Otto Finsch se rend à Vadsö pour assister à la pêche des Balénoptères, et il en rapporte une tète de foetus, conservée dans la liqueur. D'après les notes et les dessins faits sur les lieux par le savant naturaliste de Brême, nous avons décrit les caractères extérieurs de l'animal dans les Bulletins de l'Académie 1.

Dans la suite la tête du fœetus a été étudiée avec un soin particulier par M. l'abbé Gérard Smets; il a fait connaitre toutes les particularités des os, du crâne et de la face 2 .

Le professeur Möbius a publié une notice sur une Balanoptera Sibbuldii, échouée, le 26 juin 1881, entre les îles Sylt et Föhr (mer du Nord, côte occidentale du Jutland). L'animal était encore en vie quand il est venu à la côte.

1 Notice sur la grande Balénoptère du Nord, Bulletins de l'Académie, 1875 .

3 Ann. Soc. scientif. de Bruxelles, 1885. 
Plusieurs naturalistes se sont rendus dans ces dernières années au nord de la Norwège, pour y assister à la pêche des Balénoptères; ils en ont rapporté de précieux matériaux pour l'histoire de la grande espèce: en 1877, MM. Aurivillius et Forstrand, et depuis, MIM. Tycho-Tollberg, Ed. Van Beneden, Pouchet, Guldberg et, en dernier lieu, M. O. Cooks.

M. Tycho-Tollberg a publié un travail intéressant sur la structure et le développement des fanons de la grande espèce. Ils sont larges à la base, noirs comme du jais avec un teint bleuâtre.

Des expositions, comme celle qui vient d'avoir lieu à IIambourg (1881), ont, dans une large mesure, contribué au progrès de la cétologie. Ce n'est que dans des oceasions pareilles qu'on peut comparer les squelettes de ces grands mammifères, dont on trouve bien rarement plus d'un exemplaire dans un musée. M. Guldberga rendu compte de cette exposition dans le journal anglais Nature 1, et, à cette même occasion, le Dr II. Balan a publié une notice sur les Baleines principales de l'océan Atlantique et leur distribution dans cette mer 2.

Ce qui a le plus contribué à nous faire connaître les diverses espèces des Balénoptères, et surtout celle qui nous occupe, ce sont les pêcheries que l'on a établies d'abord dans les eaux d'Islande et que l'on a continuées ensuite sur les côtes de Finmark, où elles ont pris une grande extension dans ces dernières années.

\section{SYNONYMIE.}

Comme nous venons de le voir, c'est le mème animal qui est désigné sous le nom de Balcena physalus, par Pallas; Cuvierius et Physalus Sibbaldii, par Gray; Pterobalana gigas, par Eschricht; Pterobalana gryphus, par Jul. Münter; Balanoptera latirostris, par Flower; Balænoptera Cuvierius ou Carolince, par Malm.

1 No 799, 19 fév. 1885.

2 Segelhandbuch für den Atlantischen Ocean. XIV hapitel. 
Les Groënlandais le connaissent sous le nom de Tumnolik; les Islandais sous celui de Steypireydr; les Norwégiens sous celui de Blaahval.

Sars avait attribué le nom de Hushval à la Balcenoptera Sibbaldii. M. Guldberg, conservateur du Musée zootomique de l'Université de Christiania, croit que Sars s'est trompé; le Hushoul ferait son apparition dans ces régions à une autre époque que le Sibbaldii, qui ne se montre qu'en été.

Dans notre mémoire sur la Baleine des Basques 1, nous avons d'ailleurs dit que ce nom était donné à la Balcena biscayensis.

Le Physalus antarcticus de $\mathrm{Malm}$, dont nous avons vu des vertèbres au Musée de Stockholm, provenant du détroit de Magellan, représente sans doute le Sibbaldii dans l'hémisphère austral.

Parmi les ossements provenant de l'expédition française au Cap Horn et qui sont conservés au Muséum à Paris, il y en a sans doute qui se rapportent à cette même espèce.

Les baleiniers américains et anglais parlent souvent d'une Balénoptère sous le nom de Sulfurbottom; c'est, d'après eux, un animal de grande taille et qui hante l'océan Pacifique et la mer des Indes. Ils lui accordent une taille de 80 pieds et des fanons fort larges et d'un noir luisant. Nous avons ainsi plusieurs raisons de croire que ce Sulfurbottom est aussi synonyme de $B$. Sibbaldii.

Ce qui nous confirme dans ce rapprochement, c'est l'examen, que nous avons eu l'occasion de faire à Vienne, des fanons désignés sous ce même nom par le capitaine Charles Scammon et que le prof. Steindachner avait lui-même rapportés de SanFrancisco.

\section{CARACTERES.}

Cette espèce se distingue des autres Balénoptères par le rostre, qui est fort large, surtout sur le milieu de sa longueur;

"Histoire naturolle de la Baleine des Basques; Mémoires de l'Académie, 1886, p. 2 . 
par les os nasaux tronqués en avant, et par les palatins fort larges; le maxillaire supérieur est dépassé par le maxillaire inférieur, et l'apophyse coronoïde est haute et pointue; les vertèbres sont au nombre de soixante-trois ou soixante-quatre; les côtes sont au nombre de quinze ou seize paires; le sternum est large et court, les métacarpiens et les phalanges sont comparativement longs.

Les fanons sont courts et fort larges à leur base, d'un noir foncé à reflets bleuâtres 1 .

La nageoire dorsale est petite, pointue et rapprochée de la nageoire caudale. Elle est placée au-dessus de l'anus, vers les quatre cinquièmes de la long̨ueur du corps. Les nageoires pectorales sont longues et pointues. La peau du dos est d'un brun foncé tirant sur le vert. La peau du ventre est grisâtre, argentée.

Ies poils, au nombre d'une trentaine, sont placés au menton dans un espace circulaire (Malm).

Le caractère si important de la largeur du rostre, qui lui avait valu le nom de latirostris, ne se trouve cependant bien prononcé que chez l'adulte. Nous avons été à même de comparer des photographies de têtes de foetus, de jeunes et d'adultes, et nous avons pu nous assurer que la largeur des maxillaires, vers le milieu, se montre seulement après la naissance.

La taille de l'animal adulte dépasse 80 pieds. Pallas lui accorde 84 pieds.

La femelle de 1827, trouvée morte en mer, avait 83 pieds $\left(26^{\mathrm{m}}, 60\right)$.

Scoresby en accorde 82 à une Balénoptère venue à la côte d̀ Shetland pendant l'hiver 1817-1818. Il n'indique pas le sexe de l'individu.

- Les fanons sont noirs aussi dans la Balanoptera borealis, mais leur forme les fait ressembler plutôt à des fauons de Baleine qu’à des fanons de Balénoptère; ils sont en effet très étroits à leur base. Les barbes sont blanches et fines dans la Balaroptera borealis, noires et épaisses daus la Balernoptera Sibbaldii. 
Celui de Sibbald, capturé en 1692 à l'entrée du Firth of Forth, était un mâle de 78 pieds.

Un autre individu, de sexe femelle, capturé également dans le Firth of Forth, avait 80 pieds, et le foetus qu'elle portait en avait près de 20.

Knox accorde 84 pieds à un animal capturé le ă octobre 1831 près de Nort-Berwick et dont le squelette est au Musée d'Édimbourg.

Un animal échoué sur les côtes à Plymouth mesurait 79 pieds.

La Balénoptère, dont la nageoire pectorale avait été envoyée par Holböll à Copenhague, avait été prise dans la baie de Baffin le 12 août 1843. Elle avait 34 aunes danoises. A en juger par sa taille, c'était sans doute une femelle.

II. Guldberg estime la taille au moins à 73 pieds à l'âge adulte. Les plus forts individus qu'il ait vus avaient 84 pieds. On en cite de 86 et même de plus grands.

Le $D^{r}$ Finsch a vu prendre à Vadsö une femelle pleine qui avait 84 pieds.

Un pêcheur des côtes de Finmark a capturé en 1883 : le 12 juillet, une femelle de 85 pieds; le 24 juillet, une de 82 ; le 9 août, une de 81 ; le 6 juin, une de 72 et le 24 août, un mâle de 75 pieds. Ainsi, le 24 août il prend un mâle et une femelle, et la femelle a 7 pieds de plus quele mâle.

Il paraît que l'on ne prend plus de si grands individus.

Dans ces derniers temps, six individus capturés dans les eaux d'Islande ne mesuraient que de 70 à 80 pieds.

La Baloenoptera Sibbaldii est l'espèce la plus commune dans ces parages. M. Alfred Cocks rapporte que sur 406 cétacés à fanons, capturés en 1883 sur les côtes de Finmark, il y avait 175 Balcenoptera Sibbaldii, et sur le restant à peu près la moitié Balcenoptera borealis et musculus. Sur 40 individus capturés par un seul pêcheur, il y avait 19 Sibbaldii, 8 Megaptera et le restant des Musculus 1.

Les cétacés en naissant ont à peu près le tiers ou le quart de

Alrr. Cocks, loc. cit, p. 20. 
la longueur de la mère. Les Phoques, si l'on en croit le témoignage de quelques naturalistes, sont dans le même cas, et on cite même des exemples de jeunes animaux de cet ordre qui, en naissant, auraient eu à peu près la moitié de la taille de leur mère. Ce sont donc les mammifères dont la taille, relativement à la mère, est la plus grande à la naissance.

La grande Balénoptère, Balcenoptera Sibbaldii, a été étudiée par le professeur Sir Turner : le savant professeur d'Édimbourg a vu une femelle de 80 pieds, échouée sur les côtes d'Écosse, qui renfermait un fnetus à terme de près de 20 pieds; c'était done le quart de la taille de la mère.

M. Henri Berd a vu sur les côtes d'Islande un foetus qui avait 18 pieds.

Le Musée de Leipzig a reçu de Vadsö un fotus de 18 pieds également.

Un foetus recueilli sur les côtes d'Islande et envoyé par le capitaine Bottemanne au Musée de Leyde, avait 17 pieds.

Le I) Finsch a trouvé dans une femelle, à Vadsö (Finmark), entre le 7 et le 10 juillet, un fotus de 7 pieds et un autre de $41 / 2$.

Le 7 août, on a trouvé dans une femelle, capturée à l'est du cap Nord, un foetus de 9 à 10 pieds. La mère avait 70 pieds norwégiens, ou 78 pieds 2 pouces anglais.

M. Alfred Cocks a observé sur les lieux (également Finmark), le 21 juillet, un fœetus de 3 pouces, à Eretiki; un second de 9 à 10 pouces, le 7 août, à Jarfiord; un troisième de 15 pouces, à Vardö, le 14 août, et un quatrième de $13 ้$ pieds 6 pouces, du 15 au 17 août (1884).

On connait plusieurs autres fotus. La plupart viennent de Vadsö. On voit des différences de taille assez grandes dans des fotus recueillis à quelques jours d'intervalle. Il est à remarquer que l'on ne commence la chasse qu'au mois de mai sur les côtes de Finmark et que tous ces fœetus sont recueillis pendant les trois mois d'été.

Sars a figuré un fœetus de 1 pied 4 pouces.

Guldberg a rassemblé la mesure de 28 fœtus, mais on n'a malheureusement pas tenu note de la date de leur capture. 
Tycho-Tollberg a eu un embryon de 1 mètre, un de 2 mètres, un de 3 mètres et un de $4^{\mathrm{m}} \breve{\text { ร̆ }}$.

Le foetus de Longniddry avait au delà de 19 pieds.

Guldberg cite un foetus de 23 pieds, qu'il considère comme étant à terme.

Il croit devoir fixer la taille de la Balanoptera Sibbaldii, en naissant, entre 23 et 24 pieds, ou $71 / 2$ à 8 mètres 1 .

\section{ORGANISATION.}

Plusieurs travaux importants ont paru sur l'organisation de la Balénoptère de Sibbald, et parmi eux nous pouvons citer particulièrement le beau mémoire de Sir W. Turner, qui a eu à sa disposition une femelle de 78 pieds 9 pouces en chair et un fotus de 19 pieds 6 pouces.

La tête du fotus de 7 pieds, conservée dans la liqueur avec les

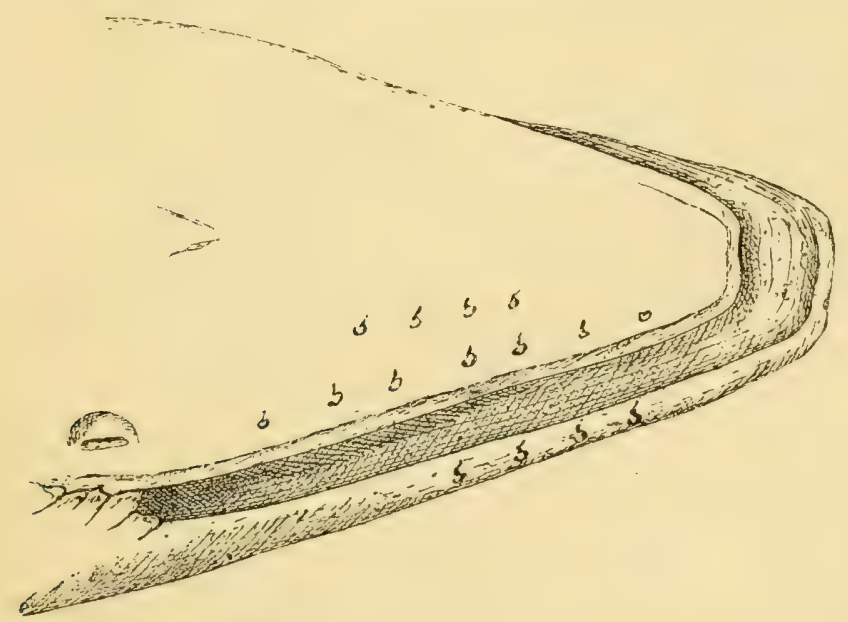

Vue de la partie antérieure de la tète du foetus, conservée à Louvain.

- Pour donner une idée de crtle dillérence de taille, nous ferons remarquer que ia petite Rostrata a, d'après Eschricht, 9 pieds de longueur en venant au monde, et la grande qui nous occupe en a 20. 
parties molles, nous a montré combien le maxillaire inférieur dépasse le bout du rostre; une double rangée de bulbes à poils garnit la lèvre supérieure, et une double rangée se montre également sur le bord et au-dessous de la mandibule inférieure.

En disséquant la tête du fœetus, nous avons observé une disposition bien curieuse dans la cavité de la bouche. La langue forme en avant une saillie; mais en arrière, au lieu de s'élever et de s'insérer sur le corps de l'os hyoïde, elle se déprime et il se forme une véritable excavation.

La caisse tympanique mesure 13 centimètres en hauteur; la grande apophyse, 24 en longueur.

La colonne vertébrale de $B$. Sibbaldii compte un plus grand nombre de vertèbres que celles des autres Balénoplères, mais il est à remarquer que l'on conserve bien rarement les dernières caurlales. Il n’y a que les naturalistes qui attachent de limportance à conserver tous les os, petits ou grands, ossifiés ou cartilagineux.

Knox a compté soixante-trois vertèbres, qu'il répartit en cervicales, sept; dorsales, quinze; lombaires, seize ; caudales, vingt-cinq.

C'est le même nombre que dans le jeune squelette de Götenborg, seulement il y a une Jombaire de moins et une caudale de plus. Cette différence peut dépendre de la dernière lombaire, qui est comptée quelquefois avec les caudales.

Pallas n'accorde que soixante et une vertèbres à la Balcena physalus, comme il appelle la Sibbaldii.

Un squelette du Musée de Stockholm, provenant de Vadsö, montre soixante-deux vertèbres, d'après une lettre de M. Smith. Ce même nombre se trouverait dans une mère et dans un foetus.

Le squelette de Hull, de 50 pieds de long, a soixante-quatre vertèbres ( 7 cervicales, 16 dorsales, 41 lombaires et caudales).

La femelle échouée dans les sables de l'île d'Oleron, en 1827, et qui n'aurait aussi que $5 / 4$ pieds, portait, d'après les suppositions, soixante-trois vertèbres ( 46 présentes et 17 qui manquaient dans la queue). 
Le squelette d'Édimbourg a soixante-trois vertèbres ( 7 cerv., 15 dors., 16 lomb. et 25 caud.).

Celui de la Balcenoptera Carolince de Götenborg, en a cinquante-six ( 15 dors., 15 lomb, et 26 coccygiennes). Sans doute, elles ne sont pas toutes conservées, puisque le nombre ne s'accroît pas avec l'âge, pas plus que celui des fanons.

Une femelle de 13 mètres, du Musée de Kiel, a également soixante-quatre vertèbres ( 7 cerv., 16 dors., $15 \mathrm{lomb}$. et 26 caud.).

L'atlas de la femelle de Stockholm présente à sa face postérieure tous les caractères que MM. Flower et Reinhardt ont attribués à cette espèce.

Pour se faire une idée de la taille des vertèbres, nous ferons remarquer que l'axis mesure, d'un bout de ses apophyses à l'autre, $1^{\mathrm{m}}, 2 \mathrm{o}$.

Nous avons vu au Musée de Stockholm une vertèbre lombaire du détroit de Magellan, dont les apophyses n'étaient pas soudées et dont le corps mesurait en largeur 49 centimètres, en hauteur 39 et en longueur 27 , avec une apophyse épineuse, mesurée de sa base, de 77 d̀ 78 centimètres.

Malm a écrit sur l'étiquette de cette vertèbre du Musée de Stockholm : Physalus antarcticus ; elle est figurée dans son mémoire 1.

Une autre vertèbre, de grandeur colossale également, une dorsale, la onzième, du même Musée, nesure en travers 48 centimètres, en hauteur 39 , en longueur 16 , avec une apophyse transverse, mesurée depuis le canal vertébral, de 54 centimètres.

Les épiphyses sont soudées.

Nous avons tout lieu de croire que ces vertèbres proviennent de l'espèce représentative de notre Sibbaldii, ou de la Sibbaldii même.

Nous venons de recevoir une omoplate d'un individu, qui avait près de 80 pieds et qui mesure un mètre soixante, de l'angle antérieur à l'angle postérieur de l'os.

2 Hvaliljur i Steriges Muzeer. Kongl. Svenska Velenskaps-Akademiens handlingar, Stockholm, 18i1, pl. 1, 7, c. 
Le sternum de jeune animal le plus intéressant est celui du Iusée de Leyde. Il consiste dans un large disque, sans échancrure en avant et sans appendice en arrière, ayant en travers de 39 à 40 centimètres, et d'avant en arrière 25 à 26 centimètres.

Le sternum du jeune mâle de Götenborg présente la même forme.

Le sternum adulte de Leyde se termine en arrière en pointe arrondie, et mesure en travers 67 à 68 centimètres.

Celui d'Ostende lui ressemble aussi bien qu'au sternum d'Édimbourg.

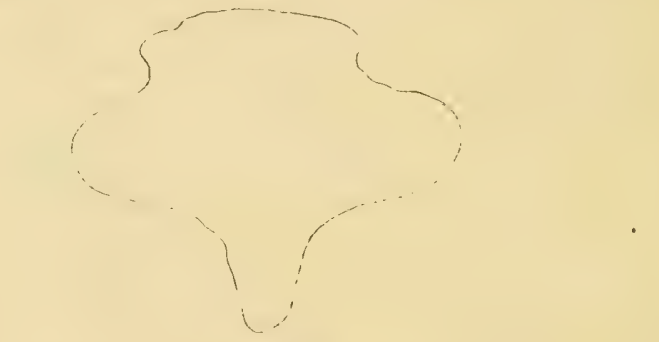

Sternum d'une femelle, du Musée de Stockholm.

Le sternum de la femelle de Stockholm se termine de la même manière postérieurement, mais, en avant, le bord n'est pas arrondi comme dans les autres; il y a pour ainsi dire un second étage.

D'après ce que nous venons de dire, cet os n'est point échancré en avant dans l'espèce qui nous occupe, cornme il l'est dans d'autres espèces.

Le sternum représenté par sir Turner, provenant d'une femelle adolescente, ne ressemble pas complètement aux autres.

Le professeur de l'Université d'Édimbourg a publié aussi la figure du sternum d'un fœtus mâle, fort intéressant; il est formé d'un cartilage qui ressemble au sternum de l'animal adulte, et d'un second cartilage fort petit, placé derrière l'autre sur la ligne médiane, et qui semble devoir former la pointe postérieure de cet os. 
Le squelette a seize paires de côtes quand elles sont toutes conservées. La dernière se perd souvent. Nous en comptons seize dans le squelette de Hull et dans quelques autres squelettes.

In fotus femelle du Musée de Stockholm n'en a que quinze.

Dans la Balénoptère qui nous occupe, on voit souvent cette première côte formée de deux pièces plus ou moins complètement soudées.

Dans le squelette d'Ostende, la première côte est réellement bifurquée, comme nous l'avons déjà fait remarquer, et celui qui l'a monté a attaché cet os à la dernière cervicale et à la première dorsale; c'est ce qui avait fait dire à Dubar, qui a décrit ce squelette, qu'il n'y avait que six vertèbres à la région cervicale.

La première côte du squelette d'un jeune mâle de Götenborg est simple, ainsi que la première du squelette de Stockholm.

Un radius des Antilles, au Musée de Bordeaux, mesure plus d'un mètre de longueur.

Dans le squelette de Hull, nous royons les phalanges des doigts au nombre de 4, 6, $\check{y}$ et 3 . Dans celui de Götenborg, décrit par Malm, ils sont $\check{8}, 8,8$, 4. Dans celui de Copenhague, qui vient de la baie de Baflin, nous avons compté $6,6,7,4$.

Il est à remarquer que les doigts des adultes sont rarement complets, ce qui paraît dépendre des luttes que ces animaux ont eu à soutenir aric les Orques. Les bords des nageoires sont très sourent rongís, aussi bien des nageoires caudales que des nageoires pectorales. Le dessin publié par Schlegel dans la Fauna Japonica montre bien comment ces organes sont entamés sur leurs bords.

D'après le fotus de Longniddry, le nombre de rangs de fanons serait de trois cent trente-cinq.

L'espace qui les sépare est d'un dixième de pouce. Chaque rang de fanons compte vers le milieu jusqu'à sept, huit et même neuf lames en avant, seulement cinq en arrière; le nombre s'accroît jusqu'à trente, mais dans ce cas les derniers sont à peine distinets.

Les fanons du foetus n'ont pas cette belle couleur noire des adultes. 
Quand le fotus a 2 mètres, les fanons sont en voie de formation. M. Tollberg a figuré les fanons d'un foutus de 3 mètres, et d'un autre qui avait atteint $41 / 2$ mètres.

Dans ces dernières années, un beau travail a paru sur la formation des fanons de la Balonoptera Sibbaldii, par Tycho-Tollberg 1 .

Sous le rapport morphologique, les fanons correspondent aux papilles du palais des Ruminants; leur origine est épidermique.

Lorsque l'embryon a 2 mètres de long, l'épithélium du milieu du bord du maxillaire s'épaissit, et cette épaisseur s'étend ensuite en avant et en arrière. Comme Eschricht l'avait déjà remarqué, le nombre de fanons ne change pas dans le cours du développement.

MII. G. Pouchet et Chabry ont exposé le résultat d'observations intéressantes sur l'évolution des dents de la Baloenoptera Sibbaldii, dans les Comptes rendus de l'Académie des sciences".

II. I'lanteau a fait connaitre des observations sur la muqueuse de l'utérus, d'après des pièces rapportées de Vadsö par le professeur Pouchet 3.

\section{MOEURS.}

Chaque espèce de Cétacé se comporte d'une manière particulière en mer, et les pêcheurs les reconnaissent à distance sans se tromper. La Balénoptère qui nous occupe est reconnaissable non seulement à sa nageoire dorsale, qui est très petite et placée fort en arrière, mais également à son high spout and conspicuous size, comme disent les baleiniers anglais.

1 Tollberg-Tycho, Sur la structure et le développement des Baleines (Bal. Sibbaldii), Archiv. Zooz. experim., t. III, n5 5otes, p. xxxix. Nov. act. Reg. Soc. Scientiar., Upsaliensis, vol. XI, 1883.

20 février 1882 .

- Planteau, Muqueuse de l'uterus de Balæoptera Sibbaldii. Journ. D'Anat. ET DE PHys., juillet, 1881, p. 277. 
La Baleine franche parcourt un espace de 9 à 10 milles d̀ l'heure, et les Balénoptères, surtout la grande, nagent encore plus vite; le capitaine sir Turner accorde à la Balenoptera Sibbaldii, qui atteint 89 pieds, une vitesse de 12 milles à l'heure, comme au Cachalot.

Le professeur Sir Turner, dans une conférence donnée dernièrement à Édimbourg, a parlé de la force des grands Cétacés. Il estime la force de la Balanoptera Sibbaldii, qui parcourt 12 milles à l'heure, dont le poids est de 70 tomnes et l'envergure de la queue de 18 à 20 pieds, il estime cette force, d'après II. Henderson, à 1430 chevaux.

Les Balénoptères sont généralement considérées comme ichtyophages, ainsi que les Mégaptères, mais l'espèce qui nous occupe ne paraît guère manger que des Crustacés.

Le Crustacé qui fait sa pâture principale est un mysidé, que l'on désigne sous le nom de Roeger; c'est une Thysanopoda, qui porte maintenant le nom de Euphrusia inermis. C'est le Krill des pêcheurs 1. La Balénoptère poursuit ces Crustacés schizopodes jusque dans les Fiords, dit Sars. Ce petit crustacé parait en si grande abondance à l'est du cap Nord, pendant les mois d'été, qu'elle sert de pâture non seulement aux Balanoptera Sibbuldii et borealis, mais également au Gadus virens et aux nombreux oiseaux marins qui descendent comme des nuages sur cette proie.

Collett a frouvé communément de 3 à 400 litres de Thysanopoda dans leur estomac. Mon fils en a ouvert à Vadsö en 1882, qu'on venait de capturer et il a trouvé leur estomac également plein de ce petit crustacé.

C'est par erreur, sans doute, que Holböll cite le Mullotus arcticus, et Pallas, les Loligo et les Méduses, comme nourriture principale de cette Balénoptère.

D'après le $\mathrm{D}^{\mathrm{r}}$ Guldberg, la Balcenoptera borealis poursuit la même pâture et ne se rend cependant pas autant à l'est que la Balcenoptera Sibualdii. Il faudrait en conclure que ce n'est pas

- Nalurwo. lerein., mái 1885. 
exclusivement la recherche de la pâture qui les guide dans leurs pérégrinations.

Le Cestode, que nous possédons à l'état de strobile, provient sans doute de ce crustacé Schizopode.

Ces Cétacés vivent par couple et la femelle atteint une taille plus forte que le mâle.

Malmgren a vu deux grandes Balénoptères, sans doute mâle et femelle, à la latitude de $79^{\circ}, 40$.

En septembre 1881, on a trouvé une femelle morte sur les côtes près de Plymouth, et le 2 novembre suivant, on a vu échouer un mâle à peu près sur les mêmes côtes.

Comme les autres espèces, la Balanoptera Sibbaldii s’apparie sans doute à certaines époques de l'année et les sexes, comme les individus qui composent les bandes, se prêtent assistance en cas de danger.

Les pêcheurs ont plus d'une fois remarqué que des Balénoptères vont au secours des individus capturés, et continuent leurs évolutions autour du carlave remorqué, jusqu'à ce que le danger d'être prises elles-mêmes devient trop éminent.

Y a-t-il une époque des amour's, ou ces cétacés s'accouplentils à toutes les époques de l'année? Est-ce dans l'Atlantique pendant l'hiver, ou dans la Mer de Barentz pendant l'été? Qu'elle est la durće de gestation? Y a-t-il un lieu de prédilection pour la mise bas? Ce sont autant de questions auxquelles il est encore difficile de répondre positivement. Nous n'avons encore que des observations isolées.

L'accouplement se fait, d'après Guldberg, en étésur les côtes de Finmark et de Laponie et il cite un exemple à la date du 13 juillet (I883). On a vu un mâle et une femelle tous les deux sur les flanes, s'approcher lentement l'un de l'autre, puis se tourner ventre contre ventre. Le vapeur tira sur le mâle, qui lâcha la femelle sans être blessé. Le lendemain on captura une femelle de $\mathbf{7 0}$ pieds. A l'autopsie, la muqueuse vaginale était rouge et injectée. L'impression était que la femelle était fécondée. Guldberg n'a pas trouvé de spermatozoïdes dans les mucosités utérines. 
Mais à en juger par la taille des fotus que l'on recueille assez régulièrement pendant l'époque de la pêche, l'accouplement doit aroir licu igyalement ailleurs et en d'autres temps; ainsi cette Balénoptère n'aurait pas d'époque fixe pour l'accouplement, par conséquent non plus pour l'époque de la parturition. Dailleur's M. Guldberg est arrivé à la même conclusion, comme il ressort d'un de ses derniers travaux 1.

La durée de la gestation dure au delà d'une année, et sans aucun doute elle varie d'une Balénoptère à l'autre.

La Balonoptera sibbullii se reproduit tous les trois ans; les Balconoptera musculus et rostrata semblent se reproduire tous les deux ans environ.

La taille des foetus, recueillis à Vadsö et dans les ports roisins, est très variable, comme nous l'avons déjà dit plus haut.

Au commencement de juillet 1883, on amena à Srartnœs, pris de Vardö, une grande fomelle, aree les mamelles pleines de lait et une matrice énorme; il a semblé au conservateur du Husice Znoturinique de Christiania, que cette femelle arait mis bas depuis peu de temps.

La femelle de la baie de Forth, ainsi qu'une autre citée par'

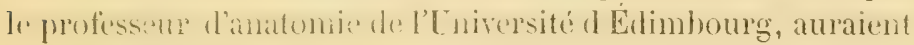
all contraire mis bas au mois d'octobre ou de novembre.

\section{DISTRIBUTION GÉOGRAPHIQUE.}

Cette Balénoptère fait régulièrement son apparition sur les côtes d'Islande et de Finmark au printemps, et elle disparaît ì la fin de l'été. Quelle direction prend-elle alors pour passer les mois d'hiver? Traverse-t-elle l'Atlantique pour se rendre à la côte de Labrador? Nous fondons grand espoir sur l'initiative,

1 G. A. Guldbeng, zur Biologie des Nordatlantischen Finuhalarlen. Znologische Jambücier, JenA, 1866. vol. 11, fase. I, pp. 126-174.

Tone XLI. 
qui vient d'être prise par la commission de pêche de IYashington, d'inviter les habitants des côtes d'informer par télégraphe le professeur Sir $\Lambda$. Baird, le secrétaire de l'institut Smithsonien de Washington, chaque fois qu'un Cétacé de: quelque importance apparaîtrait.

Pour faciliter cette tâche, M. Fréd. True, curateur des mammifères du Husée national, a publié une notice intéressante, avec dessin au trait, des différents Cétacés qui pourraient faire leur apparition sur les côtes des États-Unis d'Amérique 1.

Quant à l'époque de l'apparition de la grande Balénoptère, nous avons recueilli quelques faits qu'il s'agira de coordonner quand ils seront assez nombreux.

Sophus Hallas, qui a pris part à la pêche des Balénoptères sur les côtes d'Islande, dit que leur apparition dans les eaux d'Islande a lieu vers le 21 avril.

D'après les pêcheur's, les Balcenoptera Silbaldii et musculus, ainsi que la Hegaptera boups, apparaissent dans le voisinage du cap Nord en mème temps que la Mullotus villosus, la nourriture favorite des liades. Des millions de Larlus (Larus glaucus et eburneus) suivent ces bancs de poissons.

L'Eclipse, partie de Peterhead, le 20 avril 1886, pour la pèche à la Baleine, a rencontré, quatre ou cinq jours après son départ d'Lcosse, une Balenoptera Sibbuldii; du moins, R. Gray suppose, d'après la taille, que la Balénoptère qu'ils ont aperçue appartient à cette espèce. Le huitième jour, l'Éclipse s’est approchéc des glaces, et deux autres indiridus de la même espèce se sont montrés à l'horizon.

Le capitaine de l'Éclipse a vu ensuite des Balcenoptera Sibbaldii à côté d'Hyperoodon, et même dans des eaux qui avaient une température au-dessous de deux degrés et demi, à côté des Mysticetus. Nordenshiold croyait que les Sibbaldii n’entraient pas dans des eaux au-dessous de cette température.

1 Fred.True, Suggestions to the keepers of the U. S. Life-Saving stations .....to the best means of collecting and preserving specimens of whales and porpoises. Washington, 1884. 
A $79^{\circ}, 15^{\circ} \mathrm{N}$, , le capitaine Gray a vu encore une Bulenopter' Sibbaldii avec deux jeunes.

Arec les baleiniers, on peut dire qu'elles sont Cold w'ater whales.

Il n'est pas douteux que cette mème Balénoptère ne se montr" de l'autre côté de l'Atlantique. Le professeur Cope cite quatre espèces, dont une est comnue des baleiniers sons le nom de Finbach whale, et mesure au moins 74 pieds, à en juger par un squelette du IIusée de Cambridge. C'est le Sibbuldius sulfureus de Cope.

Guldberg cite le cas d'un individu capturé en Europe, qui portait un harpon semblable ì ceux qu'on emploie de l'autre côté de l'Atlantique, probablement des côtes du Labrador.

Yous sarons que cette Balénoptère apparait régulièrement au printemps an détroit de Daris, quand la Baleine franche l'a quitté, et elle se rend dans la mer de Baflin à de hautes latitudes. Ciest l'espèce la plus boréale du genre, dit I'. liray. Les Esquimaux, comme les baleiniers, ne distinguent pas la Balleneptera Sïbulliii de la Bulenoplere musculus, dit R. Brown I.

Nous conniissons un exemple intéressant de cette capture, faite le 12 a aut 1813 dans la baie de Baftin : c'est celle de la femelle de 3 taunes danoises, dont la nageoire pectorale avait été envoyée à Eschricin. Le ventre de cette Balénoptère était noir et gris, disait Il lböll; souris-foncé entre les plis et la nageoire dorsale petite 2 .

Celles qui doublent I caly Yord longent la cute de Finmark et se dirigent vers l'est. II. A. Cochx a vu des Balcenopler'a Sibbuldii jusqu'au 18 septembre à l'entrée de la mer Blanche.

Déjà en 18:32,0. Heuglin avait signalé à la Société royale de géographie de Londres :" que ces animax sont extrêmenent abondants dans la mer Blanche, et il avait mème exprimé l'espoir de voir l'industrie de la pêche sétablir dans cette mer, si

1 The Znologist, février 1887.

2 Escuncist, je Traité, p. 151.

s Journ. roy. grogr. Sociely, vol. XXIII, p. 129). 
peu connue alors. Il prétend aussi que les côtes de cette mer intérieure sont couvertes d'ossements de ces Cétacés.

Elle se rend dans les eaux de Spitzbergen, comme dans la mer de Baffin, à de hautes latitudes. Halmgren rapporte qu'il a ru, eomme le capitaine Gray, le 17 septembre 1861, deux lialénoptìres de grande taille, it $79^{\circ}, 13^{\prime}$ latitude nord, entre l'île d'Amsterdam et le Spitzberg. Leur navire y était à l'ancre.

On sait que cette Balénoptìre est également commune à Beeren-Eiland et sur les cottes de la Nouvelle-Zemble.

Dans le courant du nwis d'août, ces animaux abandonnent res régions, et la fremiire espuce qui quitte les coites de la Laponie, c'est la Buldenoptere Sibbaldii; la seronde espice, c'est la Bulcnopteru musculus. La Derfaptera reste la derniere, paraît-il (A. Cockx).

le lis siptembre, on a vu encore un individu de cette espèce it Eretiki.

M. Ciullburg a fait le relevé des lalénopières de cette espère capturées depuis dix ans : en 1876, il y en a eu 42; en 1877, 22 seulement, c'est le plus petit nombre; 1878 , il y en a eu 70 ; en 1879, 84; en 1880, 62; en 1881, 221. C'est le plus haut chifre que l'on ait atteint. En 1889 et 1883 , on estime? le nombre à 200 ; "n 1884 , les animaus étaient giands, mais moins ahondants; ('n $1880 \%$, on n'rn a pris que :is. On a capturé ¿t la place une rentaine de Bulinoptera boreulis. En 1886, on en a capturé une centaine. La Bulenoptere borenlis était rare cette année, mais la Bulanoptert musculus, par contre, fort abondante.

M. Alfred Cockx estime que sur 406 captures faites on $\mathbf{1 8 8 3}$ sur les côtes de Finmark, il y avait 1730 Balenoptera Sibbaldii.

La Balcenoplera Sibbaldii a été rare en 1883 sur les côtes de Finmark. Le Crustacé qui forme sa pâture, la Thysanopoda inermis, a fait défaut, et le Seje ou Cod whale des Norwégiens a paru à sa place.

$\Lambda$ cause de son énorme taille, cette espèce est plus exposée il échouer que les autres, et nous avons plusieurs exemples d'individus trouvés flottants en mer. 
Ascanius signale l'exemple d'une femelle de 60 pieds qui est venue se perdre sur les côtes de Norwège.

Le 28 juillet, on a vu sur les côtes est de l'Islande un Steypireydr flottant en mer.

Un animal de 80 pieds a échoué sur les côtes de IIollande (Oosten et Sluysche Gat ) le $1^{\text {er }}$ mai, nous ne savons de quelle année. Le commissaire De Witte fait mention des fanons noirs, d'une aune de longueur, et dont il estime le nombre à deux cents ${ }^{1}$.

Lne des captures, dont on s'est le plus occupé, est celle faite 'Il 1827 d'une femelle trouvée flottante en mer et remorqu'e par les pêcheur's jusqu'au port d'Ostende. L'animal mesurait,

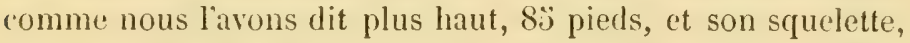
après avoir été exhiloé dans les principales capitales de l'Europe, est conservé, dans un triste état, au Jardin botanique de St-I'étersbourg 2. C'est la propriété du Musée impérial de l'Académie des sciences de $\mathbf{S}$-Pétersbourg.

Cette même année, au mois de mars (le 10), une jeune femelle, qui n’avait que ð̈̊́ pieds, a échoué dans les sables de l'île d'Oleron, et on peut se demander si ce n'est pas un Baleineau qui avait accompagné la Balénoptère dont nous venons de parler. Le $\mathrm{D}^{\mathrm{r}}$ Fischer donne des détails fort intéressants sur cette Balénoptère : le sternum était petit et plat, les verlèbres, au nombre de quarante-six, sans celles de la queue, qui sont au nombre de dix-sept, ce qui fait en tout soixantetrois vertèbres. Les os n'ont malheureusement pas été conservés.

Le $\mathrm{D}^{\mathrm{r}}$ Fischer fait mention d'une autre Balénoptère qui a ćchoué en avril 1863 sur les côtes de Dunkerque, et qui mesurait 30 mètres.

1 Le 1 er mai est venue échuuer une Baleine: " na myne gissinge omtrent 900 git zwarte tande, een elle langh ende hreed, groot van muyle, daer wel erne gemeene schuyl ingaen soude, in der waerheyt een monster der hellen.

${ }^{2}$ "Das grosse Skelet der bei Ostende gestıandeten, welches Europa durchwanderte, ist jetz Eigenthum unseres Museums, " m'écrivait le Dr brandl dans une lettre datée de S`-Pétershourg le fer janvier 187 ?. 
On cite encore un animal, rejeté par la mer à l'état de cadavre sur la plage de Soulae, dans le golfe de Gascogne. C'était le 13 juillet 1879 . On ne voyait flotter qu'une masse informe de chair en putréfaction; la têtr et la queue étaient séparées; il y a lieu de croire, m'écrit le capitaine Jouan, que, sous l'impulsion des vagues, qui sont terribles sur cette côte, le corps aura été replié en deux. Le corps a été mis en vente, et c'est la commune de Soulac qui la achété pour 90 franes. L'animal avait 24 mètres de long 1 .

On en a olservé à diverses reprises sur les côtes d’Écosse et de Shetland.

Sibbald, qui a fait tant de bomnes observations sur les grands Cótacés des côtes d'Erosse, parle d’un mâle de 75 pieds qui a péri en 1690 dans la baie de Forth. C'était un individu de la grande espèce.

C'est encore sans doute un animal de cette espèce qui a échoué à lentrér de lllumber, an mois de septembre 1750 , et dont Scoresby fait mention.

Neill fait mention sualement d'un grand individu, dont le sexe n’est pas déterminé, qui a péri dans la même baie en 1808.

Scoresby parle d'une Balánoptipe de 82 pieds qu'il a vue sur lesertes de Shetland pendint l'hiver de 1817-1818. Elle était venue y échouer.

Arthur Jacols fait mention d'une femelle de 70 pieds, venue à la côte, à l'ouest de l'Irlande, au mois d'avril 1825.

Un jeune animal de $\$ 1$ pieds, dont le squelette est conservé au Musée de Liverpool, a échoué dans la Dee.

Une femelle trouvée morte en mer, a été remorquée à Plymouth le 27 septembre 1831 ; elle était Iongue de 79 pieds (Couch) et son estomac renfermait une grande quantité de Pilchards. D'après sa taille, ce serait une Sibbaldii, mais cette espèce se nourrit-elle de Pilchards? N'y a-t-il pas de l'exagération dans la longueur? C'est ce que nous croyons plutôt.

1 Act. Soc. Linn. de Bordeaux, séance du 6 aoù 1879. 
En octobre 1831, un individu a échoué à l'embouchure de la baie de Forth; son squelette est au Musée des sciences et des arts à Édimbourg. Il avait 76 pieds (Knox).

En 1833, une bonne capture a été faite dans le Humber, près de Hull ; le squelette de l'animal est conservé au Musée de cette ville.

Le professeur Sir Turner, qui signale cing échnuements, parle d'un individu échoué à l'entrée du Firth of Forth en 1830.

Une Balcnopfera Sibbaldii, prise, le 9 novembre 1869, a l'entrée de ce fleure, et qui heureusement est tombée dans de bonnes mains, nous fournira des renseignements bien importants. Elle s'était montrée en spectacle aux habitants de la côte pendant quatorze jours. C'était une femelle sur le point de mettre bas, et dont l'acquisition a été faite de manière à être utilisée complètement pour la science. Le professeur Sir Turner a d'abord publié une première notice dans les Proceedinys de la Société royale d'Édimbourg, puis, dans une notice particulière, il a fait connaître le sternum 1, le placenta ainsi que l'os du bassin de la mère et du fortus mâle qu'elle portait.

Le fœetus n'avait pas moins de 20 pieds 6 pouces; la mère mesurait 78 pieds 9 pouces.

Dans cette notice, il fait savoir qu'au mois d'octobre 1869 une autre femelle avec son jeune, appartenant d̀ la même espèce, a été capturée sur la côte de Shetland, mais il ne nous apprend pas si cet animal a été conservé pour la science.

Le même savant parle encore d'un autre individu échoué à Wick (Caithness) en 1871.

La Balcenoptera Sibbaldii pénètre dans la Baltique.

Nous avons déjà parlé de deux mâles rencontrés dans cette mer intérieure, le $\delta$ avril 1825 et en juillet 1862 , et que Jul. Münter a fait connaître.

Le 29 octobre 1865 , un individu måle, non adulte, a été cap-

- Turner, On the sternum .... of the longniddry Whale (Balænoptera Sibbaldii). JouRn. OF aNATOMY and Parsiology, vol, IV. 
turé près de Göteborg, et a été décrit par M. Malm sous le nom de Balanoptera Carolina. C'est le professeur Flower qui reconnut le premier que ce jeune mâle est une Balchoptera Sibbaldii. Son squelette est conservé au Musée de Göteborg.

Le 26 juin 1881, entre les îles Sylt et Föhr (Baltique), les douaniers aperçurent une femelle Balenoptera Sibbaldii de 80 pieds, échouée sur le sable; elle fut tuée à coups de hache. Le $\mathrm{D}^{\mathrm{r}}$ Möbius acheta le squelette pour le Musée zoologique de Kiel. Le bassin manque 1.

Nous ne croyons pas que la Balanoptera Sibbaldii soit confinée dans l'Atlantique septentrionale, comme les autres espèces du genre; elle hante le Pacifique comme l'Atlantique, et se répand même dans les eaux de nos antipodes.

On parle, en effet, sous des noms divers, d'une grande Balenoptère, répandue dans l'Atlantique comme dans le Pacifique, dans la mer des Indes comme dans la mer du Japon, et dans les eaux de nos antipodes, comme nous l'avons déjì dit dans les généralités sur le genre.

Les baleiniers américains et anglais parlent souvent d'une Balénoptère sous le nom de Sulfurbothum; c'est un animal de grande taille et qui hante l'océan Pacifique et la mer des Indes. Nous avons plusieur's raisons de croire que ce Sulfurbottum appartient à l'espèce qui nous occuppe. Ils accordent en effet à ce Sulfurbottum une taille de 80 pieds et des fanons fort larges et d'un noir luisant.

Au mois d'août 1871, une jeune Balénoptère a échoué près de l'embouchure du Rio de Lozan, un des affluents du Rio de la Plata. A en juger par ses fanons noirs, par la face inférieure du corps, également noire, un peu plus claire que le dos, mais pas blanche, nous croyons que c'est une Balcenoptera Sibbaldii. Son squelette est conservé. Burmeister lui a donné le nom de Balcnoptera intermedia.

A. Smyth a vu des Balénoptères de 9̋̈ pieds à Table Ba!y.

1 Prof. Möвrus, Ueber einen bei Sylt gestrandelen Blautual (Balænoptera Sibbaluii). Naturwiss. vereins für Schleswig-Holsteın, Bd. VI. Kiel, 1865. 


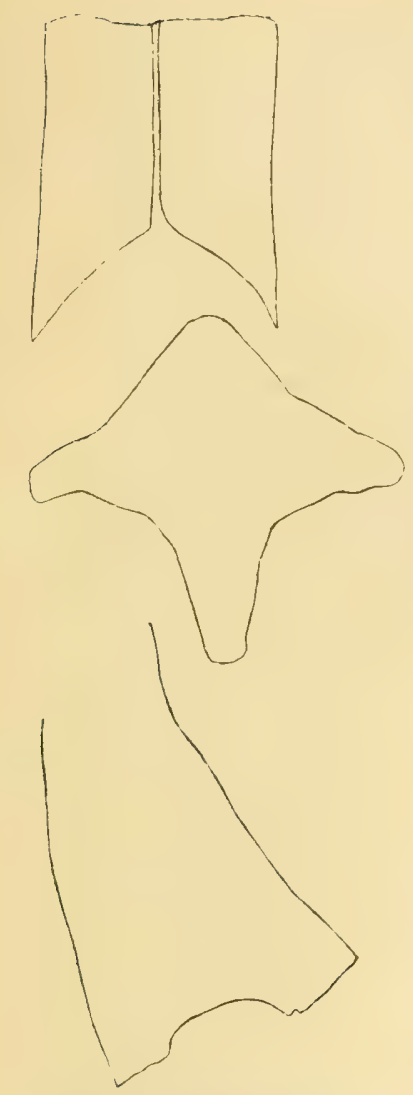

Os nasaux, sternum et partie distale de la première côte.

Le Muséum d'histoire naturelle de Paris a reçu de Patagonie des squelettes qui se rapportent à la Balcenoptera Sibbaldii et à la Balcenoptera musculus. Nous reproduisons ci-contre la première côte qui est bifide, les os nasaux et le sternum.

Un de ces squelettes a été trouvé sur la plage dans le NewYear-Sund, à la Terre de feu. L'animal dont il provient avait échové sur la plage, et les Fuegiens s'étaient empressés d'enlever la chair pour la manger.

\section{MUSÉES.}

Jusque dans ces dernières années, on ne connaissait dans les Musées aucun squelette bien authentique de cette espèce, et quelques os séparés de grande taille pouvaient être attribués à des géants de l'espèce commume.

Aujourd'hui le doute n'est plus possible, surtout depuis que l'on possède des ossements et des squelettes des Balénoptères recueillis aux pêcheries d'Islande et de Finmark.

Nous allons indiquer par ordre alphabétique les IIusées qui possèdent des restes authentiques de cette Balénoptère.

Au Musée de l'Université de Breslau, on conserve le squelette d'un jeune animal qui a péri, en 1862, au Sund.

Le Musée de Bruxelles possède aujourd'hui un squelette complet, provenant des pêcheries de Vadsö. Il est d'une remar- 
quable conservation pour les membres comme pour les os du bassin.

Il possède également une vertèbre isolée, dont l'origine n'est pas connue, et qui se rapporte sans doute à cette espèce. Le corps est haut de 32 centimètres, large de 39 centimètres, mesuré au milieu du disque; l'apophyse épineuse mesure :2 centimètres depuis le corps de la vertèbre, et elle est loin d'itre complète. C'est une des dernières lombaires.

Le Musée de Copenhague possìde une tête et divers ossements provenant de la pèche en Islande (1866). Le professeur Reinhardt a publié une notice intéressante sur ces dernières pièces, avec de bonnes figures. Depuis 1843, on y conserve également le squelette d'une nageoire pectorale d'un animal capturé au mois d'août dans la mer de Baffin. Cette pièce a été envoyée à Eschricht par le gouverneur Holböll.

Au Musée de l'Tniversiti de Christiania on conserve un squelette d'un mâle de 77 pieds, préparé par Guldberg, en 1881.

L'Indian Museum de Calcutta renferme plusieurs ossements importants.

On trouve dans la ville d'Edimbourg des ossements de quatre individus différents : il y a d'abord un squelette complet d'une femelle qui a échoué en octobre 1869 sur les côtes de Shetland. Il est monté au Iuscée de l'Ĺniversité. Ce squelette fut déposé d’abord au Jardin zoologique d'Édimbourg. II s'y trouve ensuite des vertèbres cervicales, dorsales et lombaires, avec des côtes et une omoplate recueillies sur les côtes de Shetland. Le squelette d'un individu de 84 pieds trouvé floltant en mer près de North-Berwick, le 5 octobre 1831 , étudié par Frederick Knox, est conservé dans le Département d'histoire naturelle du Musée des sciences et des arts.

Le Musée de Göteborg renferme un squelette d'un jeune mâle qui a échoué, le 29 ortobre 1865 , non loin de cette ville, et dont Malm a donné une belle description, sous le nom de Balcenoptera Carolinoe. 
On conserve un squelette de mâle au Musée de l'I'niversité de Greifswald, recueilli à l'île de Rügen en 1820̆.

Un squelette est conservé à la Société philosophique de Hull, provenant d'un animal de 47 pierls de long, qui a été capturé en 1833 dans le Humber.

Le squelette d'une femelle de 13 mètres de longueur' qui a péri, en juin 1881, sur les côtes du Jutland, est conservé au Musée de l'Université de Kiel, grâce aux soins du professeur Möbius.

A la Rochelle, on possède deux vertèbres, dont l'oriģine est inconnue, et qui proviennent sans doute de cette Balénoptère.

Le. Musée de Leyde renferme également deux premières côtes d'un mâle de 78 pieds anglais, un sternum, un atlas ef un axis, puis quelques os provenant de la pêche d'Islande, donnés par le capitaine Bottemanne.

A Leipzig, on conserve un fortus de 18 pieds, conservé dans le sel, que l'on a reçu du cap Nord.

Le Musée de l'Université de Liège est en possession de deux beaux squelettes, l'un de mâle, l'autre de femelle, tous les deux adultes; ils proviennent d'individus capturés dans l'établissement de pêche de Vadsö.

Le même Musée possède également des foctus, dont un a une dizaine de pieds de longueur.

Le Musée de Liverpool possède des vertèbres, des côtes et des fanons rapportés d'Islande par M. IIenri Baird en 1868.

Nous avons à Louvain la tête, les nageoires pectorale et caudale d'un foetus de 7 pieds de long, que le Dr Finsch a rapporté de la pêcherie de Vadsö, ainsi que l'omoplate dont nous avons déjà parlé.

Le British Iuseum possède également un squelette; il provient de la collection de Lidt de Jende d'Utrecht. C'est la vue de ce squelette qui avait frappé II. Flower en 1864; son coup d'œil exercé lui fit reconnaître une forme nouvelle, à laquelle il donna le nom de Balcenoptera latirostris.

On conserve aujourd'hui un autre squelette au British Museum, qui provient d'une femelle de 79 pieds, capturée ì 
Plymouth; des caisses tympaniques et des fanons de la Nouvelle-Zélande.

Au Muséum de Paris se trouve aujourd'hui un squelette de mâle et un autre de femelle, tous les deux rapportés des pêcheries de Vadsö par le professeur Pouchet.

Il s'y trouve également depuis longtemps une omoplate et un atlas de grande taille dont l'origine est inconnue.

Le même Musée est en possession aujourd'hui l'un squelette provenant du cap Horn 1.

Le plus ancien squelette est celui de l'animal qui a été trouvé, en 1827, mort en mer, par les pècheurs, et qui a été remorqué jusqu'à Ostende. Comme nous l'avons dit plus haut, ce squelette, après avoir été exhibé dans les principales capitales de l'Europe, est allé échouer au Jardin zoologique de Saint-Pétersbourg.

Pallas parle d'un squelette de 8 t pieds de long (pieds anglais), provenant d'un animal capiuré dans la mer Glaciale, sous le nom de Bailcua physalus; il a été envoyé par Petrus Kargin, en 1740, au Musée de S'-Pétersbourg. La taille et la couleur des fanons (laminis atro-taruleis) ne laissent guère de doute sur l'espèce. Personne na pu nous domner des renseignements sur ce squelette pendant notre séjour à St-Pétersbourg.

On voit encore au IIusée de $\mathrm{S}^{\mathrm{t}}$-Pétersbourg un atlas et trois vertỉbres lombaires, provenant rles côtes de la Nouvelle-Zemble, que nous attribuons à cette espèce.

A la fin de 1878, le Nusée de Stockholm a reçu de Vadsö un squelette de Balanontera Sibbuldii femelle et de son foetus à peu près à terme 2 .

Au Musée de l'Académie des sciences de Stockholm, nous avons vu en outre une vertìbre dorsale (deuxième), rapportée par le $\mathrm{D}^{\mathrm{r}}$ Kinberg des côtes de Patagonie (détroit de Magellan);

${ }^{1}$ La Revue scienlifique, dans son $n^{\circ}$ du 29 mars 1884, rend compte di. l'expédition scientifique au cap Horn et fait merition de ces ossements qui ont été rapportés par la Romanche.

2 Saitt, Ueber Balcenoptera Sibbaldii. Zoologiscier anzeiger, 16 décembre 1878 . 
elle est d'une dimension extraordinaire. Nous n'en avons pas vu de plus grande. M. Malm rapporte encore au même animal plusieurs autres vertèbres, dont deux Jombaires. II. Malm a fait le relevé des Cétacés conservés dans les Musées de Suìde; il a donné à cette espèce le nom de Physalus antarcticus.

Nous connaissons aussi quelques squelettes conservés dans Ies Musées hors d'Europe; ils sont généralement désignés sous d'autres noms. A défaut de pièces assez nombreuses de comfaraison, nous ne pouvons pas aftirmer aree certitude que c'est la même espèce qui vit aux deux hémisphères, mais nous pouvons assurer qu'elle représente notre grande Balénoptère par tous les caracteres essentiels, la taille, la forme de la face, la couleur des fanons, le nombre des vertèbres et des côtes, ete.

Il existe un squelette de Balénoptìre de 7.4 pieds au Musée: de Cambridge (Etat-L'nis) et qui appartient sans doute à cette Balénoptère. C'est l'espèce la plus grande ef la plus commune, dit le professeur Cope.

On en cite une autre de 84 pieds, capturée en 18301 , à la latitude de $19^{\circ} \mathrm{N}$, on Juggu or Amherst Islet. Ouelques-uns de ses os sont conservés au Musée du collège médical de Calcutta, sous le nom de Balanoptera indica 1.

En 1873, une Balénuptère de très grante taille a échoné sur la côte sud-est d'Otago: ses fanons, d'après le Dr Coughtrer, sont semblables a ceux de la Balcenoptera Sibbuldii des mers arctiques a et ont la largeur et la couleur de la Balcenopterr Sibbaldii d'Europe.

Le squelette décrit et figuré dans les Trans. of the Yew-Zealand Institute, sous le nom de Physulus australis, par M. Hector, provient d'un animal capturé au sud de Cook's Strait; il a soixante-quatre vertèbres 3 ; il est conservé au lfusce de Wellington.

1 Buytr, Journal of the Asiatic Society of Bengal, xxvin, 1860.

2 Captain F. H. Hutron, Trans. n. Zel. inst., vol. VII, p. 266.

${ }^{3}$ Le texte porte 57 , mais M. Hector a mis 64 dans un tiré à part qu'il a cu la bonté de me faire parrenir. 
Un squelette d'un animal de 90 pieds, dont les fanons avaient 28 pouces de longueur, 18 de largeur, et tout noirs, est conservé au Musée de l'Université de Melbourne. L'animal a échoué sur la côte de Vittoria 1.

On conserve des fanons bien authentiques de cette espèce à Edimbourg (de Shetland), à Liège (de Finmark), à Louvain (de Shetland), à Viemme et à San Francisco, de Sulfurbothum (du Pacifique).

\section{DESSINS.}

Il existe quelques anciennes figures de Balénoptères, mais comme l'espèce n'a été définitivement reconnue que dans ees dernières années, on n'a pu donner des figures authentiques que dans ces derniers temps.

La plus ancienne figure, pensons-nous, est celle que: Rob. Sibbaldus $\approx$ a publiée dans sa Phalainologia nov'a.

Van Breda en a publié une d'après le corps recueilli en mer en 1827 par des pêcheurs d'Ostende, et dont le squelette se trouve à $\mathrm{S}^{\mathrm{t}}$-Pétersbourg 3 .

Diffirentes gravures et deux lithographies de grand format sont conservées à la Bibliothèque royale à Bruxelles.

Nous trouvons cette espèce représentéc dans le grand atlas de Goldfuss, vol- 4, pl. 332 4.

Fried. Rosenthal a publié une lithographie de l'individu échoué en $182 \partial$ dans la Baltique.

Le professeur Münter publie deux planches représentant l'animal vu des diver's côtés $ّ$.

Eschricht possédait un dessin de la Balcenoptera Sibbuldii.

- Gigloli Celacei osservali, r. 5ै1.

- Phalainologia nova. Edinburgi, 1692, pl. III.

5 VAN Breda, Afbeelding van den op 5 nov. 1827 gestranden Walvisch re Oostende. Letterbode.

$\downarrow$ Goldfuss, Atlas, vol. IV, pl. CCCXXXII:

5 Müxten, Ueber Zwei in 19 Jahrh. bei Greifswald männlichs Individuen von Bal. Sibbaldii. Greifswald, 1877. 
Burmeister a figuré l'animal, vu de profil, par sa face inférieure et par le ventre, faisant ses évolutions dans l'eau 1, sous le nom de Balonoptera intermedia.

Sars a publié d'abord, en 1865 , le dessin d'un mâle et des détails sur le squelette 2 , puis celui d'un animal adulte 3 et d'un foetus mâle de 1 pied 4 pouces de long.

En 1874, le zélé professeur de Christiania a publié le dessin d'une femelle adulte des côtes de Finmark. Le dessin de la planche III représente un individu capturé à l'établissement du capitaine Foyn. La planche IV représente un fotus de 9 pieds et demi 4.

Le $\mathrm{D}^{\mathrm{r}}$ Malm a publié plusieurs photographies faites d'aprìs le jeune mâle qui est allé échouer dans le voisinage de Gütenborg, le 20 décembre 186:. Il reproduit vingt-deux photographies sur dix-huit planches, deux planches lithographiées et trois gravures sur bois :. Le savant Directeur du Musée de Göteborg figure également la région cervicale avec les deux premières dorsales, l'atlas et l'axi:', les quatre premières côtes avec l'hyoïde, et le squelette du membre pectoral.

Le professeur Sir Turner en a donné aussi une très bonne figure 6 .

Nous arons publié un dessin de cet animal, d'après les extraits du Journal de royage et les croquis que le Ir Finsch avait rapportés de Vadsö, en 18737.

- Allas de la Description physique de la république Argentine. Bueno:Ayres, 1885.

- Vid-Selskabets Forhandl., for 1865.

Vid-Selskabets Forhandl., for 1878.

+ Sans, Om Blaahvalen (femelle adulte), Christiania Vid-Selskab. FoliHANDL. for 1874. Sars, Forsalte Bidrag lil kundskaben om vare Bardehvalu: Ciristiania videnskabelsk. Forhande., 1880.

5 Malm, Monographie illustrée de la Balénoptère.... Stockholm, 186\%.

- Turner, On account of the great Finner Whale (Balænoptera Sibbaldii), Trans. Ror. Soc. Édinburgh, vol. XXVI, pl. V, 1870 et lllust. London News, $1869, \mathrm{n}^{\circ} 1567$.

7 VAN Beseden, Nolice sur la grande Balénoplère du Nord (Balænoplera Sibbaldii), Bull. Acad. roy. de Belgique, 2* sér., t. XXXIX, 1875. 
Le cap. Scammon a publié un dessin du Sulfurbottum, ou Sibbuldius sulfureus Cope, pl. XIII. Comme Burmeister, il représente l'animal faisant ses évolutions dans l'eau.

Du Bar a publié plusieurs planches représentant grossièrement le crůne, vu de trois côtés différents, les mandibules, l'os hyoüle avec la caisse tympanique, le sternum, la colonne verlébrale, l'atlas, une première côte avec deux autres, l'os du bassin, l'omoplate et les os de la nageoire pectorale.

Le professeur lieinhardt a reproduit quelques bonnes figure's, représentant la tite avere les us nasaux, l'atlas ef le corps de l'os hyoidle, d’après les pièces ronservées au Nusée de Copenhague, provenant d'individus eapturís dans les eaux d'Islande.

Gray a figuré l’ålas ot cinc cervicales. Reinhardt a également publié une figure de l'énormu atlas rapporté par Hallas des côtes d'Islande.

Nous avons reproduit dans l'Osténgaphie, pl. XII et XIII, fig. 2.: it 3., la tite vue d'on haut, la région cervieale, la nageoire pectorale, le stermum of l'atlas avec l'axis, vus de face. Ces dessins sont faits d'apres dis piices du Musée de Copenhague et de Göteborg.

M. Smitt a eu l'obligeance ds me faire parvenir des photographies de la trite d'une femelle et du firtus, de l'atlas, du sternum, de l'hyoüle, des cotes et des membress conservés au Musie de Stockholm, pour faire la comparaison avec les mêmes os provenant de diverses localités.

\section{PARASITES.}

On commait sur cette Balénoptère de petits Cirripèdes et deux Copépodes à l'extérieur, un Mlonostrme, un Filaire, un Échinorhynque et un Cestode dans l'intestin.

On a trouvé des Penella sur une femelle de 13 mètres, capturée sur la côte du Jutland, le 26 juin 1881, et sur quatre individus capturés, en avril 186\%, sur les côtes est d'Islande.

Les Penella sont des Cirustacés parasites qui s'implantent par lis tête dans l'épaisseur de la peau, et qui se déforment si com- 
plètement qu'à moins de les avoir étudiés, ou d’avoir étudié les Copépodes, qui minent sous tant de formes différentes la peau des poissons, on ne saurait les prendre pour des animaux.

Un autre commensal est celui que l'on a trouvé d'abord sur les fanons de la Balanoptera boreculis, et qui vit également sur les fanons de la Balanoptera Sibbaldii.

Il est à remarquer que les Cirustacés, dont ces deux Balénoptères se nourrissent, sont les mèmes, d'apri's quelques naturalistes qui ont assisté à la pêche.

Creplin a trouvé des Filuria crassicandu et des Monostomum plicatum dans l'œesophage et dans l'intestin grêle.

On troure communément dans les intestins l'Echinorlynchus, Jont Malm avait cru devoir faire une espèce nouvelle, sous le nom spécifique de Brevicollis.

Le professeur Sir Turner a trouvé également cet Échinorhynque dans l'individu qu'il a disséqué.

Cette Balénoptère nourrit en mème temps un Cestode, sans doute noureau pour la sclence, mais que nous navons pu déterminer à défaut de scolex. A en juger par les proglottis, c'est en tout cas un animal voisin des Bothriocéphales.

Ce Cestode a été recueilli a Vadsö, il y a quelques années, et nous ne savons si l'on n'en a pas trouvé depuis d'autres exemplaires complets. 



\section{LES}

ZIPHIOÏDES

DIES

MERS D'EUROPE,

PAR

P.-J. VAN beNeden.

HÝRIRRE DE L'ACADÉMIE BOYALE DE BELGIQUE.

(Présenté à la Cilasse des sciences dans la sénce du 7 janrier 1888:

Tome XLI. 



\title{
LES
}

\section{ZIPHIOIDES}

DES

\author{
MERS D'EUROPE.
}

\section{GENÉRALITÉS.}

Au commencement du siècle, en creusint les bassins d'. Anver's, les ourriers terrassiers mirent au jour des rostres de Citarés veritablement peitrifiés; ver's la meme époque, sur les bords de lat Méditerrancé, on décourrit une tête légeirement mutilée, plus ou moins fossile, arec un rostre solide; ne pouvant la rapporter, pas plus que les rostres d'Anvers, à un Cetace vivant des collections, Cuvier proposal pour les désignen. le nom de Ziphius.

Cé nom de Ziphius avait été employé, par quelques auteurs

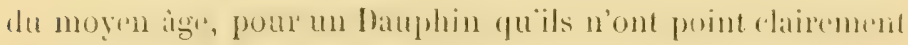
déterminé.

Les Ziphius, disait Curier, ne sont ni tout à fait des Baleines, ni tout à fait des Cachalots, ni tout à fait des Iyperoodons; ('e sont, dit-il, probablement des restes d'une nature détruite it dont nous chercherions en vain aujourd'hui les origines.

La Cétologie a fait bien des progrès depuis le jour où le grand naturaliste a écrit ees lignes, mais ses prérisions ont reegu 
une éclatante contirmation : le genre Ziphins est devenu le type d'ume famille dont quelques espèces, encore en vie, errent à laventure dans les divers Océans comme s’ils n'avaient plus de patrie. Ils sont originaires des régions chaudes, mais ils se rendent indiffíremment ver's le nord ou vers le sud, fréquentant le pôle arctique comme le pôle antaretique. On trouve, en effet, le Cachalot dans les eaux du Groenland et du Spitzherg comme dans celles de la Tasmanie et de la Nouvelle-Zélande.

Blainville qui avait ígalement au plus haut degré le sentiment des affinités, arait distingué de bonne heure les Cétacés. qui se groupent autour des Ziphims, sous le nom do Iletrirdontes.

Eschricht avait fort bien reconnu les aftinités des Ziphius avere les Hyperoodons et les Miropterons; il proposa de nommer les premiers Chenocefus, Entenwall, d'après un ancien nom danois. Ces travaux d'Eschricht ont done à leur tour confirmé l'opinion de Cuvier 1. Le savant professeur de Copenhagur réunit différentes espices autour des Ziphius, et il propose de les désigner sous le nom de Rhynchoceti. Il prend l'Hyperoodon pour type et fait remarquer que le régime de tous ces animaux est le même; tous se nourrissent de Céphalopodes.

En 180̈0, un Ziphius vint échouer sur la plage des Aresquier's. non loin de Frontignan. Paul (iervais profita de cette occasion pour montrer que le Ziphius curirostris se trouvait encore parmi les espèces vivautes; il proposa de réunir certains Cétarés, intermédiaires entre les lauphins et les Cachalots, sous li* nom de Ziphioüdes.

Durernoy, qui avait à cetté poque la direction des collections d’anatomie comparée du Muséum, füt chargé par l'Académie des sciences de faire un rapport sur le mémoire de Gervais; il passit "n revueles nombreux squelottes conservés dans les galeries du

1 "Der Entenwall (Hyperooden) und der Kleintlosser (Micropteron) stellan sich uns dennach als sparliche Leberbleibsel eincr vorweltichen grossen Altheilung der Wallihiere dar, und warseheinlich stchen sie eben desw gen jetzt so isolirt da , dit Escurıcht. 
Muséum et plaça le Cétacé de la plage des. Aresquiers, non dans 1e genre Ziphius, comme Gervais l'avait fait, mais parmi les Hyperoodons; les Cétacés étaient partagés en cinq familles par Duvernoy et il réunissait, sous le nom de Ilétérodontes, les genres virants et fossiles que nous regardons comme Ziphiö̈des. Un ne comait bien dans cette famille des Hétérodontes, ajoute Duvernoy, que les espèces du genre Hyperoodon 1.

Le profenseur Flower s'exprime, comme Cuvier et Eschricht, au sujet de l'anciemneté des Ziphiö̈les : lat raneté de ces Cétacés i léporque actuelle contraste singulièrenent avec leur abondance à l'époque de la formation du crag, et fait croire, ajoute le savant Directeur du British Museum, que les Ziphioüdes. actuels ne sont que des survivants d'ancienmes familles?.

Dans la liste des Cétacés du British Museum, publice en $188 \%$, . II. Flower admet, à côté de la famille des Balénides, une famille de Cachalots daus le sous-ordre des Cétodontes et réunit dans une sous-famille les Ziphiince, les IIypernodom, less Ziphius, les Mesoplodon et les Berardius.

Nous ne comprenons pas les motif's de cette séparation des Cachalots; ces Cétacés partagent, avec les autres Ziphiö̈des, les mêmes caractères et le mème genre de vie. La taille seule diflère.

Nous avons depuis longtemps exprimé l'avis qu'il n'y a pas the raisons de séparer les Cachalots des véritables Ziphioïdes, bien qu'ils aient de nombreuses dents au maxillaire inférieur.

Les Cétacés forment à notre avis trois familles distinctes parfaitement caractérisées, par toute leur organisation, at sperialement par leur squelette, par leur genre de vie, comme par leur répartition géographique.

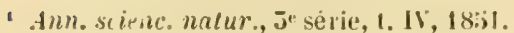

" "This comparative rarity al the present epoch contasts greally with what once obtuined on the earth, especially in the period of the deposition of the Cratg formations, and leads to the belief, that the existing Zipho ds are the survivol's of an ancient family.... "W. H. FLowe.h, On the recent Ziphioid Whales, Taxss. Zooz. Soc. of Loxnox, vol. VIII, 1871. 
Chez les Ziphioüdes, fes os de la face sallongent pour formere un véritable rostre; les yeux sont placés fort en arrière; la fente buccale est petite; les narines s'ourrent ì gauche et forment un croissant dont la convexité est en avant; la tête est peu symétrique, surtout les os qui encadrent les fosses nasales: le cràne sélive parfois tout droit ct surplombe sourent andessus des orifices des narines.

En général, les os lacrymaux 1 et mastoidiens sont séparé; les mandibules sont toujours symphisées sur une grande longueur.

II y a souvent quelques dents rudimentaires non alvéolées ¿ l'une ou à l'autre mâchoire; dans le Cachalot elles sont rarhées sous la peau au palais et completement déformées.

I la màchoine infériemere elles sont alvéolées et varient quant i leur nombre, quant ì la place qu'elles occupent et enfin quant à leur forme.

Les os de l'oreille ont des caractères communs à toutes les riperes de rette famille: les Baleines, les Balénopteres et les Mégaptères ont l'apophyse qui les attache au crâne insérée sur le rocher; les Ziphiöndes ont cette apophyse insérée sur la caisse tympanique meme; les Cefodontes n'ont pas cette apophyse developpée. Chere ces derminers, la raisse ne tient au crâne que par les parties molles.

Le corps de l'os hyoüde ainsi que les cormes sont très larges.

Les vertibres sont allongees et peu nombreuses (10i it :3i); Ies nageoires pectorales, fort petites, sont en disproportion avec la taille de l'animal; les doigts sont au nombre de cinq; les côtes sarticulent åu sternum par des cartilages, comme le professeur Flower l'a fait observer en prenier lieu chez les Cachalots, les Hyperoodons, les Ziphius et les Berardius.

Les dernières côtes sont seulement insérées sur les apophyses transverses, comme dans tous les Cétacés.

Entre l'estomac ef l'intestin, il y a une suite de six rompantiments.

"Ils nexistem fas separement dans les Cachalots. 
Le tube digestif présente une muqueuse, dirrisement alvin:lée selon les genres, et une portion de l'intestin suffit pour distinguer l'espere dont il provient. Le cecum manque.

Plusieurs Ziphiö̈les présentent des différences sexuelle; très grandes: les Cachalots diffèrent entre eux surtout par ba taille, les Hyperoodons par la conformation de lat tète, les Micropterous et les Ziphius, par la dimension des dents.

Jusqu'ici on ne comnait guère le spermaceti que dans les Cachalots; nous avons tout lieu de croire que tous les Ziphiördes en produisent; nous en avons reconnu depuis longtemps dans les Ifyperoodons, et nous venons dapprendre qu'un màle de Berardius, capturé en 1862 dans le port de Porirua (Porirur harbour), d'une longueur de 27 pieds, a produit, outre 240 gallons de fime sperm oil, me quantité considérable de spermaceti logée dans la partie supérieure de la tête.

La famille des Ziphiö̈des se distingur non moins bien par le régime: depuis longtemps Eschricht a fait la remarque que res Cétacés poursuivent surtont les Céphalopodes, et il a proposé de les appeler Theutophages, de Theutis, Cahnar. On? trouve, en effet, toujours des becs of des rristallins de ress Mollusques dans leur estomac.

Vrolik a trouvé jusqu'à dix mille bees dans l'estomar: d'un? Hyperoodon I. Jul. r. Haast a ouvert l'estomac d'un Berardius qui contenait un demi bushel de bees rornes d'octopus, tous de la mème grandeur 2 .

Parmi les caractìnes propres aux Ziphiö̈les, on peut encore? citer les sillons qu'ils montrent sous la gorge et qui correspondent avec la longue symphyse de leur mandibule.

Blainville avait déjì observé chez le Wieropteron, et IIaalland rhez l'Hyperoodon, deux paires de sillons sous la gorge entre les deux maxillaires.

Plusieurs Ziphiö̈des gémissent ou beuglent quand ils sont échoués sur la plage : fa femelle de Vieropterom qui est venue?

2 Vrolik, loc. cil.

2 V. Hast, Amn. 1. Mag nal. hisl. Octohe jazo. 
¿ la cơte à Ostende, poussait de véritables gémissements. L'Ilyperoodon qui a péri sur la côte en Bretagne, à Hillion, en 1880 , beuglait de manière à effrayer la nuit les habitants à une grande distance 1. Le Ziphiö̈de dont Jul. Haast a parlé dans une notice, reproduite par M. Flower, et qui se rapporte sans doute à un Berardius, beuglait par moments comme un taureau (bellow like a bull).

Ces animaux vivent géméralement par schools composées surtout de femelles. Les Cachalots capturés isolément ou échoués sont généralement du sexe mâle.

Les Ziphioïles virent surtout dans les mer's profondes des régions éffuatoriales, vì ils rencontrent en abondance le's Líphalopodes. On compte que ce's Mollusques ligurent dans

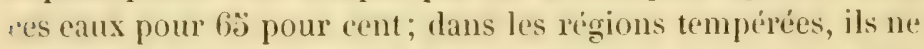
sont qu’à 30 pour cent, et dans les régions froides, on n’en compte plus que 6 pour 100. Ces Mollusques, à l'exception des poulpes, vivent toujours an large. La différence de conformation du corps indique bien l'animal pélagique "t l'animal cotier.

Les Ziphiö̈les qui ont éti signalés dans les mers d'Europe sont: le Cachalot, l'lyperoodon, le Ziphins catirostris, le Mieropteron de Sowerby. On ne connat qu'un senl individu de l'espèce qu'on a nommée Micropteron europecus.

Les Kogia que l'on a signalr's d'abord au cap de Bonne-Esprirance, puis en Australie, ont éti trouvés dans ces derniers temps dans la mer des Indes, au Japon, et dans l'Atlantique sur la côte de New Jerscy et à l'est des Florides.

Les Berardins signalés d'abord à la Nouvelle-Zélande, puis au nord du P'acitique, ne sont représentés en Europequ'ì l'état fossile.

A en juger par les ossements de Cílacés que Stejneger a rapportés du nord du Pacifique, surtout de l'lle de Behring, les Cétacés Ziphiö̈les ne doivent pas être rares non plus dans ces parages; Ie courageux voyageur que nous venons de citer,

- Bulletins, פ" sere, t. XLIX, 1880. 
at rapporté, au Mlusie de Cambridge, des restes de Berurdius, de Ziphius et de Micropteron.

Nous ferons remarquer aussi que parmi les descendants des Ziphiö̈des, autrefois sı abondants à la fin de l'époque tertiaire", dans l'Ancien comme dans le Noureau Honde, plusieurs semblent s'être réfugiés particulièrement dans les caux de nos antipodes.

Il semble y aroir un contraste entre les Cétacés vivants des régions chaudes et des régions froides; les premiers, habitant des régions équatoriales, sont généralement cosmopolites: le Cachalot visite le Groënland comme la Nouvelle-Zélande; l. Ziphins que nous arons d'abord cru propre aux Indes est le même que celui que Cuvier arait cru fossile et qui visite encor: la Méditerranée; le Vicropteron de Sowerby, reconnu dabord sur les côtes d'Angleterre et de Belgique, se retrouve également aux antipodes, tandis que les espèces véritablement polaires, comme la Baleine franche, le Narval et le Beluga, ne quittent jamais les glaces. On dirait que res derniers ont fait leur apparition après les autres et qu'ils hantent seuls les réggions glacées des pôles.

Les Ziphioïdes sont généralement mal représentés dans le's Ilusées, et il n'y a qu'un petit nombre de bons dessins des animaux et de leurs squelettes.

Les Ziphiö̈des n'ont pas été assez souvent étudiés én chair, dans des conditions favorables, pour connaitre leurs commensaux et leurs parasites. 



\section{LE ('ACHALO'T}

\section{(PHYSETER WACROCEPHALLS).}

\section{LITTERATIRE.}

Eudley, An essey upon the nut. history of Whates... (Amber gris formel in the spermacti Whalc), PHI. Trass., XXXIII, no 587, 1725, p. $2 \xi 0$.

Aibbald (nob). Phalainoloyia, Sive observationes de rarioribus quibusdam Balcenis in Scotia lillus nuper rjectis... Cum 5 lab. Edinb., 1775.

mobcrison, Description d'un Cachalol, Traxs. Pullos, 1770.

Fubielus othon. Fauna grocnlandica, in-8 , Lipsix, 1780.

enifre camper, Ostélogie du crine des Cachalols, Obserratoss axat. sur les Cétacés, p. 88.

saceson, .., An account of a Whale of the Spermaceti Trib', rast mit shore on the. Yorkshire contst, 1825.

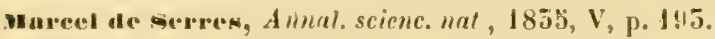

Thomas mente, A few obsereations on the natural history of the sperm Whale, in $8{ }^{\circ}$, London, 1853.

Bennet, w., On llie natural history of Pliyseter macrocephalus, Plloc. Zoor. Soc, IV, 185 li.

mianville, Su les Cachulols, Axs fravgeases ét étrang. D'anatomie ET DE PUYSIOL.0GIE, 1. 11, 1858.

D' Juckson, I. E. Dissction of a spermateti Whale, Boston, JOLRN, OF NAT, HIST., VOI. $\mathrm{V}, 184 \mathrm{Hi}$. 
owen, d history of Rrilish fossil mummals, Londun, 1846.

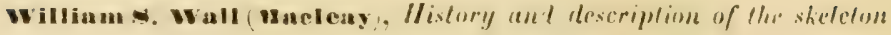
of a new sperm Whale, sydney, $18: i 1$.

owen, Stiull of a feetal Cachutot. Deseript. of the osteol. serics in the Musenm of the Roy. Coll. of surycons of Englund, t. 11, 1835.

Exacekel, Bericht über die am $1 \$$ aug. bei Cilla mova gestrandelen Poluralle (Physetcr...), Siтz. Ber. Cl. Akad, Wiss., Wien, XI, p, 765, 1835.

Hower, On the osteology of the Cachalot or sperm W'icale, 'Tiass. Zoot. Soc., London, v. VI, part. VI, 1868.

Inuxley, A manuel of the anatomy of extefrated unimals, lig. 106 , london 1871. La léte d'un fortus.

'urner, On the caplure of a sperm Whate on the corst of Argylestire. Proc. RoY. Soc. of Eombingl木, 1. 563 , session; $1870 \cdot 1871$.

rurner, Add. moles on the occurence of the sperm Whale .., Pnoc. Roy. Soc. EDswuigu, 1871-1872.

Turner, Détails sur le sternum du Cachalot cehomé ì Sliye, en 1871. Phoc: Roy. Soc., Edinburgil, 1871-1872.

Fischer, Nole sur les Cachalols échoues sur le's cóte's occeniques dr France, Joursal de Zoologie, t. I, p. 256, 1879.

Turner, Noles on some rare prints of stranded Whales, Joensur of anitomy axd Physiology, vol. XII, july 1878.

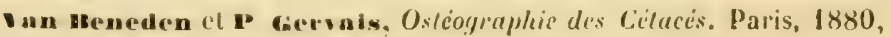
ก. $\overline{5} 0 \overline{5}$.

M.eone we sanctis. MonographinZon). analom. sul Capridoglio are nalo a Porlo S. Giorgio, R. Acanema dei Lascel, alli, $5, I X$, avec 7 pl. Roma, 1881 .

pouchet, Sur la boilc ì spermaceti, Conptes nesnes..., 4 aoủ 1884.

Poucher, De la symétrie de la face ches les Cítodontis, in 40, Paris, 1886. 


\section{HISTORIQUE.}

Ite' Cachalot a éti connu des anciens, mais fort incomplitrment au point de vue de ses attinités zoologiques.

La place réritable de ce Cítaré, à la tête des Ziphioüles, n’a été proposée que dans ces dernières années.

Presque tous les maturalistes qui st sont oecupés de Cétacés, lui ont consacré un chapitre particulier, tantôt à cóté des Baleines à cause de sa grlande taille, tantôt à côté des Cétodontes ou parmi eux à cause de ses dents.

Le Cachalot est, parmi les Ziphö̈les, ce que les Baleines ot les Balénoptèress sont parmi lés Balénides, c'est-à-dire, que ces animaux ont continué à garandir jusqu'i l'éporque actuelle. Les Géothériens, au contraire, ont atteint leur grande taille à des époques antérieures; partout ils sont visiblement à leur déclin sous le rapport du nombre et de la taille.

Albert un des premier's parle de deux Cítaces qui ont échoui en Itollande, et commo ils ont fourni du blanc: de Baleine, ce sont, selon toute probabiliti, des Cachalots dont il fait mention.

Belon et Rondelet en font également mention, mais trop vaguement pour être rertain quils ne les ont pas confondus avec d'autres Cétacés. Il en est de mêne de Gesner.

Ambroise Paré parle de ret animal d'après un individu qui a rehoué dans l'Escaut, près d'Anver's, en 1377. Il en domme un dessin, mais qui est loin d'être exact.

En 1598, un individu, échoué non loin de Scheveningur, a été l'objet d'une description faite par Clusius.

Ln siècle plus tard, Sibbald a fait mention du Cachalot, d'après un animal du sexe màle, à 42 dents, qui a péri en 1689 dans la baie du Forth, et d'une femelle qui a péri deux ans auparavant dans les mêmes conditions.

Le premier Cachalot conmu aux Etats-Innis d'Amérique, est, 
dit-on, un animal qui avait été jeté sur la cỏte sud de Nantucket 1 .

Vers 1712, un baleinier poussé au larye par un vent violent au milieu d'une gamme de Cachalots, en captura un, et c'est depuis lors que l'on a commencé la pêche en pleine mer 2.

Dans les Transactions philosophiques de 1723, Dudley fait la description d'un Cachalot qu'il a eu sous les yeux.

Anderson croit devoir admette quatre espèces de Cachalots, se fiant trop aux descriptions souvent incomplètes ou fautives des auteurs.

Artedi en admet égalemert quatre 1798 el Brisson / 17:36! en admet même sept.

Limé, comme on le comprend bien, na pas pu ćclareir l'histoire des Cachalots.

labricius (1780) a vu le Cachalet pendant son séjour sur les cotes du Groenland et a laisse une homne description de lanimal.

Déjai en 1789, les baleiniers américains du Cap Cod se rendent dans la mer de's Indes, pour faire la chasse à ces animanx; on rapporte qu’un gentleman du Cap Cod en avait apereu, à son retour des Indes, un certain nombre et avait "Mgage ses compatriotes à les poursuive dans ces parages.

En septembre 1791, doseph Russel et fils et Corn. Howland doublèrent le cap Horn à la recherche des Cachalots, et revinent heureusement du Pacifique aree un plein chargement. C'est, si je ne me trompe, le commencement de la griande peche dans le Pacifique. On pretend toutefois que le premier baleinier qui s'est rendu dans cet Ocran pour faire la chasse à ces animaux, a été envoyé, en 1787, par la Nantucliet Colony of Whalingmen from England.

1 The lirst sperm whale known in Nantuchel was foum decd, and asture on the southwest part of the lstind.

2 The lirst spermwbale taken by Nantucket whateman, was captured by Christopher llussey, about the year 1712, am the capture, cesticed to efrect a radieal change in the pursuit of this business, was the resu t of i n accicut. 
Pierre Camper a fort bien connu le Cétacé qui nous occupe:

"La forme du crime, dit-il, celle des os de la face, la structure des dents, leur emplacement dams les seules mâchoires inférieures, l'ouverture impaire des fosses nasales, la structure différente des vertibres cervicales, sont autant d'anomalies qui ne permettent pas de les confondre avec d'autres."

Nous citons ce passage pour montrer la justesse de coup d'œil du savant anatomiste hollandais.

Les Cachalots n'ont de ressemblance avec les Baleines, disait-il, que par les proportions de leur volume 1. Il figure toutefois une région rervicale de Baleine pour me région cervicale de Cachalot.

Lacépède (1804) figure le mêne animal sous le nom de Physale cylindrique, de Cachalot macrocéphule et de Cachulot trompo. Lacépède n’avait pas vu les cétacés qüil décrivait, pas mème leurs os, et i! a dû nécessairement commettre de nombreuses erreurs.

En parlant du Cachulot microps, il confond le vai Cachalot asec l'Orgue, et une partie de son réeit se rapporte à l'ennemi de la Baleine.

Cuvier, après avoir passé en revue ce que l'on arait fait avant lui, se demande si ce ne serait pas une grande témérité à lui de. gritendre qu'il ny a encore qu'une seule espèce de Cachalot. Après ce préambule, il fait la descrpition des os du squelette qu'il avait achetí à Londres, en 1818; mallemreusement, ce squelette était défectueux et, ce qui est plus grave, en le ronstituant avere des os de divers individus, le marchand lui avait domné plus de vertèbres que le Cachalot n’en possède réellement. Cuvier aurait dû s'apercevoir que ces vertèbres ne se suivaient pas régulièrement.

Les mandibules différentes, dont parle Curiex, sont, dapres ce que nous savons aujourd'hui de la taille des sexes, des mandibules de male et de femelle. On sait que la taill. des mâles a le double de celle de la fémelle. 
Fred. Cuvier parle du Dauphin dr Bayer, Delphinus Bayeri, ef à la fin de sa description il ajoute que, d'après lui et son frère, le Dauphin de Bayer de Risso est un Cachalot. D'après la figure qui se trouve dans les Actes des curieux de la nature, on voit parfaitement que c'est un Cachalot qui y est représenté, mais le dessinateur a déplacé l'orifice des évents, croyant sans doute corriger l'original.

Brandt et Ratzeburg ont reprorluit tout re que l'on a dit du Cachalot, avant 1829.

Thomas beale a fait paraitre a few observations on the nutural history of Sprerm Whale; London, 183.3. . Il aceorde 80 pieds aux plus grands individus et publie un chapitre sur leurs habitudes. Thomas Buale pritend que les Cachalots forment des gammes distinctes, les unes de lemelles, les autres de jeunes mâles, et que ces derniex's se subdivisent d'après leur àge. Ces gammes sont appelées Schools par les Anglais, et Thomas Beale dit qu'il 'll a vu de "300 ì 6000) individus. Dans chaque gamme il y at toujours quelques grands mâles fort jaloux de leurs droits, ajoute-t-il. Le's màle's adultes sont souvent isolés, à la recherehe de leur pâture; ils sont toujours plus faciles à attaquer.

Le neme auteur mentionne les plares favorites de leur capture, depuis la Nourelle-Cumé jusqu'à la Nouvelle-Hollande, le Chili, la Californie et la mer de Chine.

En 1838, Blainville publie une note sur les Cachalots; il ne sait si on doit admettre plus d'une espèce : ancun peut-ifire. saul le Cachalot macnocépHale, liest suffisamment caractérisé pour etre décidément admis comme lel. Hans cette note il fait comnaitre le Physcter breviceps, aujourdhui Kogia, d'apris une tête rapportée du Cap de Bonne-Espérance 1.

En 18:31 il a paru à sidney une notice sur l'histoire of la description d'un squelette de Cachalot monté à l'Australian Museum; elle est accompagné de deux planches. - Ceste

- Annales françuises el élrangères d'Analomir el de Physiologie, Paris, tome second, 1858, p. 353 
notice vient d'être réimprimée, et M. William S. Wall a ajouté la figure complète de la nageoire pectorale aver les os carpiens, d'après une photographie.

En 18:3, Maury a publié une carte indiquant à la fois les lieux fréguentés par le Cachalot et les Balemes, sans tenir compte naturellement des còtes que ces Cétarés visitent accidentellement. Il y a quelques régions où l'on trouve en mêm: temps des Baleines et des Cachalots. On voit, par ce tableau, que ce Cítacé ne dépasse guère, an Nord, le 50" degré de lat:tude, et, an sud, le 60", de maniere qu'on doit le considérer comme un animal des régions chaudes.

Yous avons également des publications de Claas Mulder e! de Van Bemmelen sur des individus qui sont venus se perdre sur les côtes de Belgique et de la Néerlande.

Cornalia, dans sa Fume d'llulie, a fait comnaitre deux Ciachitlots qui se sont perdus dans la Méditerranée.

Le professeur Sir IV. Turner a publié une note fort intéressante, sur quelques gravures rares, représentant des Cachalots échoués. Dans le's Proce de lu Société royale d' Élimhourg, il a publici, en 1870-1871, une noties sur la capture du Cachalot sur les côtes d'Écosse et, l'année suivante, une note additionnelle dans le même recueil.

II. le Docteur Fischer a communiqué, en 1872, dans le Jonrnal de Zuolegie de Gervais, un travail inferessant sur les Cachalots qui ont échoué sur les cotes océaniques de Franer.

II. Flower a fait connaître, avec le soin- qu'il met dans toutes ses publications, le squelette de ce curieux animal; il en a eu plusicurs à sa disposition, les uns provenant d'individus

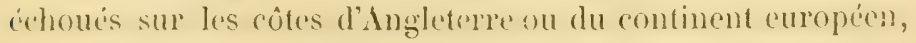
les autres provenant des mers de nos antipodes; le Musíe du College royal des chirurgiens de Londres en posside trois des côtes de Tasmanic.

Après un exposé historique de ce que l'on sait sur le squelette, le Directeru du British Museum domne qualdues notes sur l'histoire des caractères généraux et des conditions dans lesquelles se trourent : 10 le squelette de Tasmanie, qui

TOME XLI. 
est au Musée du Collège royal des chirurgiens; $2^{\circ}$ le squelette de l'animal qui a péri, en juillet 1863, près de Thurso, dans le comté de Caithness et qui est au British Museum; 30 le squelette de Yorkshire, qui provient d'un individu qui a péri en 1823 à Tunstall, et qui est conservé dans le parc de Burton Constable.

M. Flower décrit ensuite séparément le crâne et les divers os, et il accompagne la description de sept belles planches. Notre savant confrère a bien voulu nous autoriser à reproduire la planche principale dans notre Ostéographie.

Mon collaborateur, Paul Gervais, énumère les captures opérées, dans la Méditerranée, dont on a conservé le souvenir; il fait mention ensuite de cinq échouages sur les côtes ouest de France, de divers échouages sur les côtes de Belgique, de Hollande, des Iles Britanniques, et reproduit le tableau des dix captures publié par Sir W. Turner, sur les côtes écossaises; entin il cite les Cachalots dont la présence a été constatée sur les côtes de Suède et de Norwège. D'après Allen, il indique aussi les côtes des États-Unis, les parages des Iles Aléoutiennes, la mer du Japon et d'Okhotsk.

Gervais expose ensuite la composition du crủne et le système dentaire. Il décrit également les ossements fossiles du Pliocène, de la Gironde et du département des Landes.

En 1874, un Cachalot a échoué, le 10 mars, sur les côtes d'Italie et a été l'objet d'un intéressant mémoire de II. Leone de Sanctis. Ce travail est accompagné de sept planches.

MII. Pouchet et Beauregard ont publié, dans les Comptes rendus (août 1884), une notice sur la boîte à spermaceti; on serait cn présence d'une sécrétion non glanduluire d'un ordre particulier, d'après ces savants.

Pendant la campagne scientifique de l'Irirondelle (1887), le professeur Pouchet a eu l'occasion, durant un séjour de trois semaines aux Açores, d'étudier un Cachalot fraîchement tué. Nous espérons qu'il rendra bientôt compte du résultat de ses observations.

Tulled Newton fait mention de deux dents de Physeter 
macrocéphale du Forest bed, à Norfolk, et d'une région cervicale de Balanı. Ces déterminations auraient besoin d'être confirmées, la première surtout 1.

\section{SYNONYMIL.}

Physeter macrocephalus.

Catodon macrocephalus, (Gray).

Cachalot, des Français.

Sperm Whale, des Anglais.

Pott Fisch, des Hollandais.

Capidoglio, des Italiens.

Le Cachalot porte le nom de Kigutilik sur la côte de Groënland, s il faut en croire Fabricius qui en a domé une description.

\section{CARACTERES ET DESCRIPTION.}

Le Cachalot a la taille des Baleines, mais au lieu de fanons atl palais, il a le maxillaire inférieur garni d'une vingtaine de fortes dents coniques, assez espacées. Le mâle a une taille beaucoup plus forte que la femelle, aussi est-il plus redoutable. La femelle ne dépasse guère 30 pieds, le mâle en atteint jusqu ì bi) et même plus.

Lat taille parait très variable; les solitaires ou les vieux mâles deviement beaucoup plus grands. La gamme qui a péri dans l'Adriatique en 183 't ne se composait que de petits individus, probablement femelles.

Le's Cachalots d'Iudierne, qui étaient tous femelles, avaient, dit-on, une longueur de 12 à 16 mètres.

Eschricht comnaissait cette différence de taille et croyait

\& Ann. a. Ilag. nat. hist. vul. XIX, 1887, p. 239. Proc. GÉor. Suc. 1887, 1. 227 
devoir attribuer à la femelle sculement la moitié de la longueur du mâle.

Cette différence du mâle rappelle les ruminants qui vivent aussi par bandes, et chez lesquels les mâles ont à défendre le troupeau.

Le capitaine Gray a vu, au mois de mai, une femelle pleine qui n’avait que 29 pieds et qui contenait un fotus de 10 pieds. C'est donc à peu près le tiers de la mère.

Les Cétacés en général se distinguent des autres mammifìres par le défaut de symétrie, et ce défaut se fait plus particulierement sentir chez les Cachalots, comme M. le professeur Pouchet le fait remarquer dans un mémoire qu'il m’a fait l'honneur de composer, ì loccasion de mon einquantenaire de professorat ${ }^{1 .}$

Les Cachalots ont une tête énorme avec un museau carré et tronqué, à l'angle antérieur et supérieur duquel sont placés les évents; elle est renflée par suite de l'accumulation du spermaceti. La tête osseuse se distingue par sa forme excavée qui la fait ressembler à un canot.

La mandibule séloigne complètement de celle des Baleines; les deux branches se réunissent sur une grande longueur comme dans les Cétodontes ì longue symphyse.

On comnaît plusieurs mandibules de Cachalot dont l'extrémité antérieure est recourbée; il y en a une au Nuséum à Paris; Beale en cite deux cas; M. J. Murie en cite trois et II. Flower en eite encore un autre. Le Dr Fischer 2 a cherchí la cause de cette déformation qui se produit assez souvent, et qui commencerait presque après la lactation; il suppose que la déformation est due à une ostáite, et il termine son article en disant qu'il ne comprend pras clairement comment une ostéite' seule " pu produire des incurvations aussi considérables, à moins que la maladie ne soit congénitale ou presque congénitale.

2 Pocchet, M. G. De la symérrie de la face chez les Célodontes, ih-4. Paris, 1886.

${ }^{2}$ Nole sur une deformation pathologique de la macho re inferieure du Cachalol. Journal de l'axatome et de la phisiologie, no 4 , Pl. XIII. 
Ne serait-ce pas plutòt l'effet, non d'un arrêt de développement, mais d'un défaut, d'un obstacle à l'épanouissement' du bout de la michoire. Nous avons sous les yeux un maxillaire de foetus de Baleine franche : cet os a le bout antérieur parfaitement replit sur lui-mème, ce bout est comme une feuille de papier qu'on a pliée 't qui doit s'étaler ensuite; au moindre obstacle à son redressement, il conserverait la déformation, en apparence pathologique, qu'on remarque à ces mùchoires des Cachalots.

C'est un arrèt d'épanouissement et non pas de développement. L'arrêt est dû à une cause externe.

Nous avons publié dans notre ostéographie la figure d'une mandibule recourbée sur un de ses côtis et dont nous citons plusieurs exemples,

Les Cachalots n'ont point d'os lacrymal séparé, d'après les observations de II. Flower; ce qui n'est pas une raison suffisante pour les exchure de la famille des Ziphiö̈es. Cet os est probablement uni avec l'os malaire:

Les dents de Ziphioüdes different de celles des Delphinides par l'absence d'une couche d'émail. Elles manquent dans tous les Cétacés de cette famille ì la mitchoire supérieure. Dans le C'achalut, il en existe toutefois au palais, mais elles ne percent pas les gencives. Elles sont irrégulièrement déreloppées dans la peau du palais et ne correspondent aucunement à des alvéoles. M. Flower a figuré une de ces dents 1.

Owen fait mention d'une femelle dans laquelle Bennett a trouré huit dents semblables de chaque côté du maxillaire supérieur 2.

Jïger de Stuttgart a déerit et figuré une de ces dents dans un journal russe, imprimé à Moscou en 18373.

D'après Bennett, les dents subissent certaines variations; il a trouvé les nombres suivants : 21-20; 29-21; 22-22; 24-24; 22-23; 21-26; 23-24; 22-24; 19-20.

1 On the Osteologie of the Sperm-whale, p. 5200 .

2 Owex, Odonlographie, Tab. S9, lig. J-4.

${ }^{3}$ Bemerliungen.... (Nazsal et Cachatol) Moscou, 1837. 
Les Cachalots, comme tous les Ziphioïdes, ont un petit nombre de vertèbres; leur formule est : cervicales 7 , dorsales 8 , lombaires 11, caudales 24 , en tout 50 .

Les Cachalots ont leur atlas séparé et les six autres cervicales réunies.

La dernière cervicale a le corps à peine plus épais que celui des vertèbres précédentes; dans la Baleine, le corps de cette même vertèbre est notablement plus épais que celui de la sixième et de la septième. L'Hyperoodon présente sous re rapport les mêmes dispositions que le Cachalot.

Les vertèbres cervicales, à commencer par l'atlas, ont un talon qui passe au-dessous du corps de la vertèbre suivante, et ces talons donnent une grande solidité à cette région.

Ces talons existent également dans plusieurs genres de Cétacés fossiles, comme les Balamula et les Balanolus.

Les vertèbres dorsales, au nombre de huit, ont toutes la face inférieure du corps carénée et leur épaisseur n'a pas plus de la moitié de l'épaisseur des lombaires. Elles portent toutes sur' le corps la facette articulaire correspondant à la côte. Les apophyses transverses diminuent en longueur, régulièrement d'avant en arrière.

Les lombaires ont, comme toujour's dans les Cétacés, le corps plus développé que celles des autres régions.

Dans les caudales, les zygapophyses sont très éloignées du corps de la vertèbre.

Toutes les vertèbres ont des caractères particuliers qui les distinguent; les apophyses transverses sont courtes dans toutes les régions et particulièrement élevées dans la région dorsıle.

Le sternum de l'animal adulte ne forme qu'un seul os, allongé d'avant en arrière, élar'gi en avant, rétréci au milieu, et se terminant en arrière par une languette régulièrement arrondie. Il reste un trou en avant sur la ligne médiane. Jans le sternum plus jeune, on voit la réunion presque complète des deux pièces antérieures, qui constituent presque tout le sternum; puis une pièce en arrière encore séparée de la précédente, et sur la ligne médiane on voit des traces de leur sourlure. 
Le bassin est représenté par un seul os de chaque côté, comme dans les Delphinides.

M. J.-C. White a fait une communication sur deux débris de Cachalot, un maxillaire inférieur d'un grand individu et une dent fort intéressante 1.

Le professeur Ercolani de Bologne s'est occupé de la structure du pénis de ces Cétacés et croyait devoir admettre plusieurs espèces de Cachalots.

\section{MOEURS.}

Les Cétacés qui nous occupent sont essentıllement pélagiques et descendent à de grandes profondeurs dans la mer.

Le capitaine Gray assure avoir ru des Cachalots rester deux heures sans venir à la surface et dérouler jusqu'à 700 (fathoms) brasses de corde. Le capitaine Scammon en a ru plonger pendant 50 minutes et même une heure et quart.

Les mouvements de ces animaux sont très brusques et violents et ils nagent avec une rapidité plus grande que toute autre espèce de leur ordre. Lé mème capitaine Scammon estime la rapidité de leur course à trois milles à l'heure.

Le Cachalot tient sous plusieurs rapports de l'Hyperoodon; comme lui, en plongeant on le voit disparaitre seul et en revenant un instant après à la surface, on le voit entouré de plusieurs.

Les Cachalots ont de la voix; on assure avoir entendu des mugissements d'individus blessés à plus de quatre kilomètres de distance.

Les Cachalots, qui sont venus échouer à Audierne sur le sable, ont vicu vingt-quatre heures avant d'expirer et, comme nous l'avons dit plus haut, ils poussaient de véritables mugissements.

L'air expiré du Cachalot a une odeur d'une fétidité extraordinaire; cette opinion paraît très répandue; il n'est pas rare

1 Proceed. Bosion Soc., 1860, p. 222. 
Le trourer des marins soutenir arec assurance, que le contact de l'air expiré suffit pour soulever la peau aussi facilement et plus rapidement qu'un vésicatoire.

Le capitaine Jouan dit également que leur souftle a une odeur si fétide qu'elle provoque des nausées quand on la sent de près.

Les pècheurs disent que les Cachalots nagent par bandes qu'on appelle schools ou gammes, dans les régions tropicales ('t subtropicales, et on cite comme une de leur's stations favorites le côté sous le vent des Iles Galapagos.

Ils visitent aussi régulièrement la baie de Bengale, et les whools y sont formées généralement de femelles, accompargnées de leurs jeunes (Calves). Ces derniers ont de vingt ì trente pieds, disent les pécheur's, en confondant les femelles avec les jeunes.

Ces schools sont formées de quinze, vingt et jusqu'ì deux rents individus; ils ront au secours les uns des autres. C'est le motif pour lequel on prend sourent plusieurs individus dans une troupe.

On s'accorde assez généralement sur le rapport des máles at des femelles dans les schools.

Les mâles qui accompagnent ces schools sont les défenseurs naturels de la bande; aussi, au lieu de fuir à la première attique, comme les Baleines, les Cachalots se défendent vigourensement; on cite, duns les amnales des baleiniers, plus d'un exemple d'individus qui se sont vengés sur les pirogeses et sur h's hommes qui les conduisaient. M. Nougaret raconte, dans li levue des déx mondes, qu'il a vu un solitaire sur la còte Est du Grö̈nland se retourner brusquement après l'attaque, se freter sur la pirogue, la saisir par le milieu et la broyer sous sa formidable mâchoire.

Les baleiniers disent que ce sont les femelles qui se réunissent et que les vieux mâles vivent généralement isolés. La bande qui a péri au siècle dernier à Audierne était exclusivement formée de femelles, disent les témoins; deux de ce's femelles ont mis bas après leur échouement sur la plage. Cne 
d'elles domna mème denx petits, l'autre un seul. Ces jeunes Cachalots avaient une longueur de trois metres et demi.

Comme les mâles vivent plus souvent isolés, ils semblent aussi échouer plus souvent que les femeiles et e'est sans doute la raison pour laquelle, à l'inverse des Baleines, les Cachalots mâles viennent plus sourent à la côte que les femelles.

Le capitaine Gray a comparé les sexes des individus capturés en plein Océan, et pour 63 femelles il a compté 96 mâles adultes el $̋ 1$ jeunes.

Les gravures conservies al la Bibliothèque royale de. Belgique ne représentent que des mâles. Il y en a sept ou huit. Il n'y a qu'un dessin représentant un Cachalot dont le sexe est doutenx. Sur huit individus échoués sur le's côte's d'Écosse, il y arait cinq mâles et on na pas tenu compte du sexe des trois autres. (Sir 'Turner).

On a fait depuis longtemps l'observation que le's Cachalots qui échouent dans l’Atlantique septentrionale, sont le plus souvent des solitaires égarés dans le Gulfstream ì la recherche de: leur patture. Ces animaux ne trouvent plus que de rares Céphalopodes et vont mourir de misère sur l'une ou l'autre còte.

Les baleinier's rapportent que leur mode d'accouplement est le même que celui des Baleines: les Cachalots s'unissent debout dans l'eau, la tête au-dessus de la surface.

Nous ne savons s'il y a des époques fixes pour leurs amours: on voit leurs breeds at any season of the yaer, dit Jackson, qui a fait plusieurs bonnes observations sur ces Cétacés.

Le capitaine Colnett rapporte que les environs des lles Galapaģos sont, au printemps, le rendez-vous de tous les Cachalots des côtes du Mexique, de celles du Pérou et du golfe de Panama; qu'ils s'y accouplent ret qu'on y voit de jemes Cachalots qui n'ont pas 2 mètres de longueur.

C'est bien petit, 2 metres, puisque nous arons vu des femelles de la plage d'Aulierne mettre bas des jeunes qui avaient déja 3 mètres au moins.

La pàture des Cachalots est celle de tous les ziphioides: Eschricht les avait appelés arec raison Theutophages, dugenre Theuthis, Calmar. 
Toute leur pature consiste'en Cuttle fish, dit Atwood 1, et on sait que les Cuttle fish des marins sont des Calmars.

Tous les baleiniers s'accordent du reste à dire que l'estomac des Cachalots renferme toujours de nombreux restes de Céphalopodes. Hunter, Baussard, Eschricht et d'autres ont fait les mêmes observations sur des individus qu'ils ont eu l'occasion l'observer.

Les Cachalots de la gamme qui a péri en $18 \% 3$ dans l'Adriatique, avaient encore tous des bees de Céphalopodes dans leur estomac.

L'ambre gris, autrefois très estimé comme article de parfumerie, est le résidu de la digestion des Cachalots; son odeur musquée provient du Poulpe dont ils font leur pâture.

On a souvent trouvé dans l'ambre gris des becs de Céphalopodes et des cristallins, ce qui explique parfaitement leur origine. Ainsi un Cachalot capturé, en 171\%, sur les côtes de Sardaigne, renfermait de l'ambre gris dans lequel se trouvaient des becs de Sepria.

On tirait l'ambre gris des Molluques, de Madagascar, des Maldives, des Antilles et mème de Chine. Les Japonais le connaissent sous le nom d'excrément de Baleine.

Albert le Grand et avant lui Mareo Polo connaissaient parfaitement son origine, que l'on avait attribuéc tantôt à des oiseaux, tantôt à des Phoques ou à des Crocodiles, et on avait même été jusqu'à supposer qu'il provenait d'une fontaine bitumineuse sous-marine.

Les Cachalots produisent encore une autre substance sur l'origine de laquelle on a été également dans le doute; nous voulons parler du blanc de Baleine.

Le blanc de Baleine ou Spermaceti est la Cétine des chimistes; c'est un palmitate de cétyle, c'est-ì-dire une combinaison de l'acide palmitique avec l'éthal (alcool céthylique).

Cette substance grasse est surtout formée sous la peau du front et du rostre de ces animaux; nous avons déjà dit, que les

2 Atwood, Proc. Boston Soc, vil, 1860, p. 296. 
Hyperoodons en produisent également, et, comme leur huile est plus estimée, les Hyperoodons ont été l'objet d'une chasse spéciale depuis quelques années.

On sait qu'après avoir ouvert le front du Cachalot, on puise à seaux le spermacéti à l'état fluide, disent MII. Pouchet et Beauregard, et ils supposent, après l'examen de pièces envoyées des Açores, sous le nom de Racines de la boite, que le spermaceti est le produit d'une sécrétion particulière non glandulaire, qu'on pourrait rapprocher de la cire des abeilles 1 .

\section{PÉCHE.}

Il n'est pas sans intérêt de jeter un coup d'oxil sur l'époque 't les lieux où cette pêche a pris naissance; quoiqu'on désigne. sous le mème nom les pêcheurs qui s'occupent de la Baleine et du Cachalot, il y a cependant de très grandes différences dans ces deux industries.

On s'est livré pendant longtemps à la pêche de la Baleine avant de songer à la péche du Cachalot. On pratiquait la première industrie dans les régions septentrionales, où l'on ne voit que bien rarement un Cachalot.

On raconte que, en 1712 , un baleinier de Nantucket, Christopher Hussey, poussé au large par un vent violent, se trouva devant une school de Cachalots, en captura heureusement un, et, si l'animal n'avait pas de fanons, il avait des couches de graisse qui lui donnaient une valeur commerciale non moins grande que la Baleine; aussi on apprécia bien vite l'importance de cette nouvelle graisse qui se fige à la température ordinaire et dont on était loin de soupsonner la nature; on crut d'abord que c'était le sperme de ces animaux et les droguistes lui donnèrent le nom de spermaceti. De lì les baleiniers anglais ont fait le nom de spermulale.

C'est l'origine, parint-il, de cette pềche sur les côtes des États-

2 Pocchet et Benungand, Sur la boîle à spermaceli. Comptes rendug des Séaxces de l'Ácidéuie des Sciences. Aoùl, 1881. 
Unis d'Amérique. On exploita d'abord l'Atlantique et, en 1787, la Nantucket Colony of Whalingmen from England, envoya le premier baleinier dans la mer Pacifique.

Peu de temps après, en 1789, les baleiniers du cap Cod se rendirent pour la premiere fois dans la mer des Indes pour y faire cette chasse. On prétend qu'un gentleman du cap Cod, à son retour des Indes, avait vu de nombreux achalots dans la mer des Indes, et son récit avait déterminé quelques industriels à se constituer en société.

La premiere campagne de 1791 fut fort heureuse. On cite Joseph Russel et fils et Corn. Howland, qui revinrent du Pacifique (1791) avec un chargement complet.

On ne connait pas de véritable station du Cachalot dans les mer's d'Europe; on ne comnait que des échouements; les seules stations connues, où les pêcheurs se rendent encore, ce sont les régions équatoriales du Pareifique, de l'Atlantique ef de la mer des Indes. On cite particulierement les Iles Galapagos dans l'océan Pacifique, les Içores dans l'océan Atlantique, le golfe de Bengale dans la mer des Indes.

Le commandant Maury ne figure eependant pas ees régions d'une manière particulière sur sa carte et, dans ses lettres publices en 1850, il fait remarquer qu'on harponne également le Cachalot sur les côtes du Japon.

Un fait également cette pêche sur les côtes méridionales et orientales de la Nouvelle-Hollande.

La carte du savant commandant américain représente cet animal sur toute la largeur de l'océan Pacifique, des deux côtés de l'équateur. On voit seulement sur cette carte une tache blanche à l'Est et une autre ì l'(Ou'st des Iles Sandwich.

Ces animaux sont-ils régulièrement cantonnés ou visitent-ils I's divers océans, sans tenir compte des lieux ou des saisons?

Nous arons plusieurs preuves que ces animaux parcourent de grands espaces en mer : on a cité des individus, harponnés dans l'Atlantique, qui portaient encore des harpons lancés dans le Pacifique, et le commandant Maury fait mention d'une capture d’individus sur les côtes du Chili qui portaient encore le 
harpon japonais dans leurs chairs. Un autre Cachalot qui s'était 'nfui avec un harpon lancé sur les côtes du Pérou, a été pris au large sur la cote orientale des États-Unis d'Amérique. Ces Cétacés parcourent ainsi toute l'étendue du Pacifique et se r'endent mème du Pacifique dans l'Atlantique.

Maury indique leur présence jusqu'au $60^{\circ}$ degré de latitude sud au devant du cap Horn.

Sur les cotes d'Europe on n’a vu que des animaux égarés, tantit sculs, tantôt réunis en schools, mais il n'y a pas une seule cote à l'Est comme à l'Ouest de l'Atlantique où l'on n'en ait vu échouer. Et ce n'est pas seulement le plein océan qu'ils hantent, ils fréquentent également les mers intérieures, la Méditerranée comme la Baltique. Jusqu'ì présent on n'en a pas vu pénétrer dans la mer Noire pas plus que les Baleines et les Balénoptères.

II. Mengaret raconte, daus la Rerue des deux mondes, que, au mois de septembre 1863, un Cachalot apparut dans le cercle polaire, entre l'île Jan-IIeyen et la côte orientale du Groenland 1. La vigie signala le souffle du Cachalot et aussitôt on lança quatre pirogues à sa poursuite.

Cette pèche a lieu principalement dans l'océan Pacificue, mais il existe encore quelques baleiniers qui viennent tous les ans poursuirre le Cachalot dans l'Atlantique. Le Muséum de Paris a reçu récemment un squelette et il attend les parties molles d'un animal qui ont été préparées pour ses collections, par le professeur Pouchet; après avoir été chercher des squelettes de Balénoptères sur. les côtes de Finmark, le succ'esseur de Cuvier a voulu remplacer les ossements de la cour des galeries d'anatomie comparée par un squelette complet.

Le rapport de la Commission américaine, qui a fait le relevé des produits de la pêche introduits aux États-Lnis d'Amérique, depuis le 1er janvier 1804 jusqu'au 31 décembre 1876, estime, d'après la quantité de spermaceti apportée aux États-Unis, qu'il y a eu, pendant cette période, 2203,021 Cachalots capturés. II

t Revie des deux mondes, 1. LXXXIII, p. 707. 1869 
compte, d'après Scammon, que chaque Cachalot fournit 20 barils de spermaceti.

Comme on le pense bien, partout le nombre des Cachalots a considérablement diminué et il n'y a plus que fort peu de baleiniers qui se livrent encore à cette industrie.

On citait comme places principales : les environs des Açores, d'août ì novembre; les îles Bahama au nord, le golfe de Mexico, entre $28^{\circ}$ et $32^{\circ} \mathrm{N}$. et $18^{\circ}$ et $57^{\circ} \mathrm{W}$., de mars à mai; les lles du Cap Vert, en hiver, près Fernando Po, entre Sainte-Hélène et la côte d'Afrique et la côte Sud-Amérique 1.

Il parait que l'on capturait encore tous les ans vers 1860 , environ 100 Cachalots dans les eaux des Açores a tandis qu'aujourd'hui, d'après ce que m'écrit M. Franc. Aff. Chaves, de Ponta delgada, S. Miguel, ce nombre ne dépasse pas cinq ou six.

Il n'y a plus guère que les Américains qui se livrent encore à cette industrie.

\section{DISTRIBUTION GÉOGRAPHIQUE.}

Il est reconnu que c'est un animal des régions tropicales, que l'on troure surtout dans le Pacifique, mais qui est répandu depuis l'Équateur, au Nord jusqu'au Grö̈nland, au Sud jusqu'ì la Nouvelle-Zélande. Les squelettes reçus d'Australie au Musée du Collège royal des chirurgiens de Londres, et si bien étudiés par le professeur Flower, ne laissent guère de doutes sur lidentité spécifique de ees animaux : on a capturé des Cachalots dans toutes les mers et on a pu comparer à Londres des squelettes de l'Australic avec des squelettes du nord de l'Atlantique, sans qu'on ait trouvé entre eux la moindre différence.

Du reste, déjà à l'époque où Cuvier s'occupait des Cétacés, le grand naturaliste ne royait pas de différences entre les

\footnotetext{
'Axpersos, Nachrichten ron Islanil, 1746, p. 221.

${ }^{2}$ Dnouet, Éléments de la faune Açoréenne, p. 112 (1861).
} 
individus provenant des mers les plus éloignées les unes des autres.

Il est généralement admis, dit Lesson, que les grands Cétacés les plus connus sont répandus dans toutes les mers du globe, et que la Baleine et le Cachalot des mers du Nord sont identiques dans le Grand Océan, soit dans la partie qui baigne les côtes Nord-Ouest de l'Amérique, soit dans les mers du Cap Horn ou du Sud de la Nouvelle-Hollande.

Les Baleines, ajoutait-il, se trouvent, comme les Cachalots, aussi bien sous le soleil de l'Équateur qu'entre les glaces du cercle polaire.

La science a enregistré beaucoup de faits nouveaux depuis le jour où Lesson a écrit ces lignes, et ce savant ne s'exprimerait plus aujourd'hui de la même manière. Ce que Lesson dit du Cachalot est exact, mais ce qu'il dit de la Baleine ne l'est pas.

Sur sa carte intéressante de la répartition du Cachalot dans les divers océans ${ }^{1}$, le commandant Maury figure aussi la distribution géographique de la Baleine, ou plutôt des Baleines. Des deux côtes de l'Equateur, on voit, dans le Pacifique surtout, jusqu’au $30^{\circ}$ degré au Sud et au Nord, le domaine du Cachalot; puis au Nord et au Sud on voit le domaine de la Baleine; le premier habite les régions chaudes, la Baleine les régions tempérées; Maury n’a pas tenu compte de la Baleine franche, ni dans les eaux du Spitzberg, ni dans la mer de Baffin.

Le savant commandant représente, sous une couleur spéciale, pourpre, les endroits, également au Nord et au Sud de I'Équateur, où l'on a trouvé des Baleines et des Cachalots, nageant dans les mêmes eaux.

Le docteur R. M' Cormick, à propos des explorations à faire dans l'hémisphère antaretique, en parlant des richesses que possède la région du pôle sud, parle d'une quantité de Baleines de la plus grande espèce et de la plus grande valeur, particulièrement, dit-il, de l'espèce appelée Spermuceti. Il est à désirer

1 A chart schowing the favorite resort of the Sperm and Right Whale. 
que l'exploration anglaise au pôle sud ait lieu, pour savoir s'il existe, au milieu des glaces de ces régions, à côté des Cachalots passagers, une espèce de Baleine représentant la Baleine franche du pôle antarctique.

Il est done définitivement acquis que le Cétacé theu tophage qui nous occupe, vit principalement au milieu de l'Atlantique et du grand ocian Pacifique et qu'il ne trouve, au Nord comme au Sud, d'autres limites que les glaces polaires.

On l'a reconnu dans les parages du Spitzberg, auprès du rap Nord et des côtes de Finmarek; dans les mers du Grö̈nland comme dans le détroit de Davis, dans la plus grande partie de l'Occan Atlantique septentrional jusqu'à l'île JanMeyen (Nougaret), sur les côtes des Iles Britanniques, à l'embouchure de l'Elbe (1720), auprès de Terre-Neuve, aux environs de Bayonne; non loin du cap de Bonne-Espérance; près du ranal de Mozambique, de Marlagasear et de l'lle de France; dans les eaux qui baignent les rivages oceidentaux de la Nouvelle-Hollande; sur les côtes de la Nouvelle-Zélande; près du rap de Corientes, du golfe de Californie; le cap San-Lucar (Californie); les Iles Kingsmill, Marshall (eapit. Jouan); ì peu de distance de Guatemala ; autour des Iles Galapagos; à la rue de l'Ile Mocha et du Chili; dans la mer du Brésil; et enfin, sur toutes le's côtes du continent européen, même dans les mers intérieures, la Méditerranée, la Baltique et l'Adriatique.

Le Cachalot est done un animal orbicole ou cosmopolite; II. Flower a recu d'Australie, pour le Musce du Collige des chirurgiens, ì Londres, des squelettes de Cachalots, accompagnés de Globiceps et de Delphis, qui ne présentent aucune différence avec le's nôtres, de manière que la faune aquatique d'Australie ne correspond aucunement, pour les Cétacés au moins, avec la faune terrestres; nous ajouterons qu'il en est de même pour certains poissons plagiostomes. La faune aquatique ne possède, sous aucun rapport, un facies propre. Seulement certains types, qui ont disparu en Europe depuis les âges géologiques, semblent s'ètre conservées jusqu’à présent chez. nos antipodes. 
En tenant compte de tous les faits, on voit que le Cachalot "st généralement répandu dans le Pacifique comme dans l'Atlantique entre le $60^{\mathrm{e}}$ degré de latitude Sud et le $60^{\mathrm{e}}$ degré de: latitude Nord. Ce sont les limites indiquées par Maury et par Berghaus.

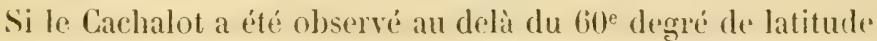
Nord et Sud, à ces latitudes on n’a vu que des individus isolés, des solitaires.

II n’a pas été vu au delà du détroit de Behring.

11 n'est pas sans intérêt de passer en revur' les individus qui sont venus échouer sur les côtes en Europee, et dont il a été temu compte par quelques naturalistes.

Les anciens ont déjà parlé de Cachalots capturés ou échoués Jans la Méditerranée.

"La première année du règne de Claude (an fi), un Cachalot (Orca) échoua sur le rivage. Il serait mort par le seul fait de son naufrage; mais l'empereur qui se mit à la tête des cohortes prétoriemnes conbattit avee cux le monstre marin. Pline, témoin de cette lutte absurde, dit-il, vit une barque submergée par l'eall dont le socrfie du Cachalot l'arait remplie. ”

Strabon parle comme Pline du Phỵseter et, dans plusieurs villes du littoral, on conserve encore des mandibules, des côtes, des vertèbres et d'autres restes de ces Cétacés.

liondelet en a vu, dans le courant du XVle siècle, des ossements qui étaient destinés au palais d'un due de Florence.

Paulus Jovius parle de deux individus qui auraient péri sur les côtes de l'Estrurie.

Ranzani parle d'un mâle de đä pieds, qui a été exposé dans le port de Pesaro en 1715 .

la même année, un Cachalot a été capturé sur les côtes de Sardaigne, mais il n'a été fait mention que de lambre "ris qu'il renfermait et dans lequel se trouverent des becs de Sépia.

Tоме XII. 
Cornalia 1, dans sa Fauna d'Italia, cite onze cas de Cachatots qui ont péri sur les côtes d'Italie : en 1713, 1768, et 177: an desà d'Ancône, en 180こ, un au-dessus d'Ancône près de fermo et un autre en 1874 à Porto-San-Giorgio, en 1810 un à Chioggia, en 1715, un près de Venise; en 1753 et 1764 sur la crote de: Dalmatie à Rovigno, en 1767 près de Zara et en 1730 prìs d. Sebenico.

Sur la côte de Nice il signale sept échouements : en 1798, 1833, 1852, 1834, 1860, 1863 et en 1864. De Gênes à CivitaVecchia on en a vu échouer trois, le premier en $186 \%$, it lit Spezzia, le second entre Livourne et Civita-Vechia, le troisiemr à Civita-Vecchia même.

Dans les Acles des curieux de la nature il est question d'un Cachalot, échoué à S'-IIospice, près de Villefranche (AlpesMaritimes), le 10 novembre 1726 .

Un autre aurait péri près d'Ascoli 3.

Mon collaborateur, F. Gervais, en cite huit échouements dans la Méditerranée.

L'Hyperoodon, échoué près de Piètri sur la côte de Toseane, dont parte Fr. Cuvier ', est un Cachalot, puisqu'il avait io pieds de long et qu'il n'y a pas d'Hyperoodon de cette taille.

En 1838, Th. Kotschy a vu, à la côte près d'Alexandrie, "u Physeter que l'on a rejeté à la mer ì cause de l'infection qu'il l'épandait autour de lui.

L'échouement le plus remarquable est celui de 18:in, le lï du mois d'août; entre Pola et Trieste, sur la côte d'Istrie, une petite gamme est allée se perdre : d'abord un jeun', puis d'autres plus grands, de 36 pieds.

Le 16, le gouverneur du littoral en vit encore deux jemnes qui lançaient, disait-il, des jets d'eau par les narines.

Le 19, la nouvelle de la prise de plusieurs Cachalots arriva it Viemne, et Heckel partit le 20 pour arricer sur les lieux le 24.

' Consalia, Fauna d'llalia. Milano.

2 BAYER, Act nat.cur, 1755, llte vol.

3 Element. di Zool, t. II, p. 6ீ 6

+ Hist. nat. des Cétacés, p. 381. 
Les cadavres étaient dépécés, mais les squelettes itaient malheureusement mèlés; le professeur Roth parvint ì "u reconstituer un. Deux têtes furent saurées pour le Musir. d'anatomie à Vieme; une tète est restée, comme souvenir, ì Citta Nuova.

Dans le golfe de Saint-Nazaire (Var), un sujet de moyemme. taille a été pris en $18 \ddot{6} 6$. Cne moitié de la mandibule en esi conservée dans le cabinet des Pères Maristes, à la Seyne, prìs Toulon 1.

Un mâle est venu se perdre, dans la nuit du 10 mars 187', it Porto-San-Giorgio et a été l'objet d'une monographie zootomico-zoologique 2 par le docteur Leone de Sanctis.

On en a vu échouer un tout près de Venise. Le dernier at iti capturé en 1874 près d'Ancône.

Dans la Bibliotheque de Ravenne, on conserve deux partie's d'un crâne, sans indication d'origine.

En Italie, M. Rob. Lawley a signalé des portions de maxillaires et des dents ayant des caracteres de Physeter provenant de Volterra et de Orciano 3.

Sous le nom de Physeter antiquus, Gervais a signalé un nouveau Cachalot dont il a trouvé la mandibule dans les sahles marins de Hontpellier. Cette mandibule est déposée au MInstum it Paris. Elle rappelle fort bien le Cachalot d'aujourd'hui.

Le professeur Turner a écrit une notice intéressante à propos d'une gravure fort rare qui représente un Cachalot, capturt 1. 2:i férrier 1601, dans le port d'Ancône. C'était un mâle. Im Iui accorde 56 pieds de long.

It fait mention aussi d'une dent, miseau jour dans des gaterie's. is lile de Sh. Ronaldsay, dont l'enfouissenent date peut-itre

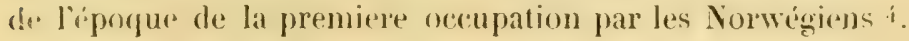

sP. Genvals, Comples rendus hebd., 184, 10.877.

2 Sul Capidogho arenalo a porto S. Giorgio, in-4n, Roma, 1881.

${ }^{5}$ Rов. Lawner, Pesi et Altri vertebrati fossili del Pliocene Tosenue Pisj, 1875, p. 11.

- Proc. of the Royal Sociely of Edinburgh, session, 1871-72, p. (iis 
En 1715, un Cachalot, long de 48 pieds, a échoué dans l'Adriatique. On en conserve des os à Sinigaglia.

Sous le règne de Louis XII, un Cachalot de 60 pieds de long vint échouer à la Selva près Collioure.

M. Guyon a même recueilli en Mgérie des ossements de Carhalot, sur la rive gauche de l'Oued-Ger, qui pouvaient fort bien se trouver la naturellement, puisque nous avons recomnu des os de Balénoptère dans les mêmes conditions. Ils ont été élevés au-dessus du nivean de la mer à la suite de soulèvements.

Paul Fischer a publié une Note sur les Cachalots échoués sur les cotes océaniques en France, dans le Joumal de Zoologic de fierrais 1. Ce's échouements ont eu lieu surtout en hiver et all printemps, de janvier ì avril. Le's principaux par ordre de date sont :

Le $1^{\text {er }}$ avril 1744, il y en a un de 49 pieds qui se perd près de. Bayonne 2. U'n dessin en est conservé dans la collection des vélins du Muséum. Fr. Cuvier l'a fait copier dans son histoire naturelle des Cétacés.

A quatre lienes de Boulogne-sur-Mer', un individu a échoué Je "̈ mars 1761, dont un dessin est conservé à la Bibliothèque. de cette ville 3 .

Le 19 janvier 1769 , un autre périt dans la baie de la Somme près Saint-Valery ‘. Rien n’en a été conservé.

En 178\%, le 1 \& mars, une school entière, composée de trentedeux individus, vint se perdre sur la côte occidentale d'Audierne כ. Lne tête provenant de cette bande a été donnée à l'Acadimie de La Rochelle par II. Domnadieu, négociant en cette ville, qui l'avait fait venir d'Audierne 6. Ine tête de sujet adulte,

1 Journal de Zoologie, 1. J, 1872, 1. 2556.

2 list. de l'Acad. roy. des sciences pour 1741 (1741).

"Fiscier, Journal de Zuologir, t. 1.

- Ballon, Calal. des Vertébrés de la Somme. (Héa. Soc. R. d’émulation DiABBEVLLE, 1853.

${ }^{5}$ Lellre de $\boldsymbol{M}$. Le $\boldsymbol{C}_{1 \mathbf{z}}$, insére dans le Mercure de France, détails citís par Lacépède, p. 205, "t par Pierre Camper, p. 98.

${ }^{6}$ Extrail du Journal Éphémérides, de M. Lambertz, végociant à La Rochelle. 
une colonne vertébrale, une portion d'os hyö̈de, un sternum. une omoplate et quelques os des nageoires pectorales, en somt conservés au Muséum d'histoire naturelle à Paris.

Nous ne parlons pas des cing Cachalots qui se sont montrís a l'entrée de la Loire, au mois de juillet 1863 , et qui sont restés, d'après les journaux de Nante's, rengagés dans le sable au reflux. Quoiqu'ils aient été exhibés à S'-Nazaire, rien n'en a été conservé et nous n'oserions affirmer' que 'ee sont des Cachalots. In d'eux mesurait, disait-on dans les journaux, $6^{\mathrm{m}}, 60$.

Vers le milieu de novembre 1872 , un màle, de 12 mètres, ust venu échouer près du phare de Biarritz. Il était en putréfaction avancée et le squelette n’a pu ètre conservé, malgré les soins de M. Souverbie, directeur du Musée de Bordeaux 1.

En 187\%, deux individus ont été vus dans les parages de (iuethary (Basses-Pyrénées). Le màle seul a été eapturé; som squelette est conserví au Musé de Bayonne; il a plus de 11 mètres de longueur.

Le protesseur espagnol Graëls de la l'az m'écrit qu'il a mis les pêcheurs à contribution, depuis le détroit de Gibraltar jusqu'à la frontière francaise, pour connaitre les grands Cétacris qui fréquentent ces paragess; ce sout la Balena biscayensis, la Balcenoptera musculus et lo Cachalot qui sont les plus connus.

On a enregistré plusieurs exemples de Cachalots qui sont venus se perdre sur les côtes de Belgique, des Pays-Bas, d'Allemagne, de Danemark, de Suède et de Norwège.

Claas Mulder estime à vingt le nombre de Cachalots commus qui se sont perdus sur les côtes des Pays-Bas; il fait avec raison la remarque que peu de restes en ont été conservés dans les Musées.

Le plus anciennement connu a échoué en 1331 sur les côte's des Pays-Bas. Houttuyn en fait mention. 11 avait 68 pieds 4 les mandibules mesuraient 13 pieds 2.

2 Genvals, Journal de Zoologie, 1. I, p. 537.

2 De Vries, Groot hist. schoumbloneel, I, p. 385;, Amsterdam, 1645. 
Le 11 mars 1566 , une femelle est venue à la côte à Zandvoort.

l.e 19 septembre 1576, il en échoue un de 14 pieds de lonģueur sur la même côte.

Ambroise Paré fait mention de trois Cachalots capturés le 2 juillet 10̈77, dans l'Escaut : un à Flessingue, un à Saflinghe, et un troisième à Hastinghe, au Doel 1.

("ítaient bien des Cachalots d'après ce qu'il dit des narines et des dents. II leur accorde ¿8 pieds. La mandibule avait de chaque côté 23 dents et on voyait autant de trous à la mâchoire supérieure dans lesquels les dites dents pouvaient se eacher.

Ambroise Paré en donne: une tigure, qui a été copiée par Aldrovande et par Johnston.

De 1898 à 1614, cinq Cachalots ont été vus sur les côtes des Pays-Bas. Quatre d'entre eux ont étó dessinés et gravés.

Emmanuel de Metteren fait mention d'un animal de 53 pieds de long qui est venu à la côte en 1601 .

Én 1606, on en cite un qui a péri à Springerplaat (île Schouwen).

l.e 28 décembre 1614, un mâle a péri à Noordwyk-aan-Zee. On en conserve la gravure 2.

En 1617, au mois de janvier, un autre mâle de 60 pieds est vonu à la côte entre Scheveningen et Katwyk. C'est de lui que provient la tête qui est conservée dans une église à Scheveningen.

Le 14 février 1619, un màle a péri à l'embouchure du Rodan; il en existe une gravure : la nageoire pectorale est représentée comme un pavillon d'oreille.

Le 12 mai 1620 on en a vu échouer un sur la côte de Poméranie (bei Carmin); un autre le 15 octobre 1640.

l.e 4 janvier 1629 , un mâle est venu à la côte à Noordwyk-

- OEuvres complètes d'Ambroise l'aré, 1. III, p. 77?, Paris, 1841.

- Pot-Walviscr, Gestrandt by Noorluzycl-op-Zce, den 28 dec. 1614. DOOR Es. VAs di VElde, in-folio. 
atall-Zue; en 1630ั, un à Scheveningen, et en octobre 1641, it péri le dernier de ce siècle. Une gravure de ces deux derniers est conservée à la Bibliothèque royale.

En 1721, au mois de janvier, un animal de 60 pieds est venu a la coute non loin de Brême, et en 1793, un échouement de phusieurs a eu lieu dans le mème endroit. Lacépède fait mention de dix-sept Cachalots qu'une tempôte violente avait poussés cette année dans l'embouchure de l'Elbe. Les moins grands avaient 13 ou 14 mètres. Il y arait huit femelles et neuf males 1.

En 17i;i;, une autre school a péri sur la côte de Mecklembourg.

Iu siede dernier, nous voyons encore quelques animaux se perrire dans les mêmes parages : en décembre 1761, il y en a deux ou trois qui viennent échouer sur l'île ou le banc \%. Civind, près de Harlingen. La caisse tympanique de l'un l'‘ux a clé envoyée à Pierre Camper ?. Il en existe un dessin sur leapel on lit: ce poisson a 73 pieds de long et 18 de haut; rist un Cachalot, échoué à l'ỉle Grin. Ce dessin se trouvail mulre les mains de Mulder et a été exécuté par P. Idserdts.

On fiat mention d'un autre qui serait venu se perdre égaleinent en décembre 1761, à Eyerland.

On cite encore un individu échoué en 1762, à T'erschelling, et un à Vlieland à la même époque de l'année.

Iin Cachalot échoué entre Zandvoort et Wyk-op-Zee, en 1762, ə) février, a été figuré par H. Spilman. 11 en existe une gravure à la Bibliothèque royale, sur laquelle on lit: Cagelol, lam! bi voeten, den 20 februari 1762, Iusschen Zandroort en "Iyk-op-Zee aangedreven.

Yous ne croyons pas que ce soit un de ceux qui sont indiqués ci-dessus.

I'après un dessin de C. van Nonrde, un màle aurait péri encore en 1764.

- Lacépede, Hist. Nat. Celaces, in-4", p. „35.

' Camper's kl. Schriften, I B, 2 st. BLfs. ANat...., p. 107, pl. XXIH. 
Un animal de $8 \mathbf{1}$ pieds, que l'on a dit femelle, a échoué le: 4 décembre 1783, sur les côtes, près de Middelbourg. On 'n possède des os dans cette ville et on en conserve un dessin ì Dornburg 1. La mandibule porte quarante-huit dents.

Ainsi, au XVI siècle, une vingtaine rle Cachalots se perdent dans ces parages : trois sont reconnus comme mâtes, un comme femelle, et on est sans renseignements sur le sexe des alltres.

Au XVIfe siecle, il s'en perd une vingtaine, mais les renseignements laissés sur leur sexe sont bien incomplets.

Au XVIII ${ }^{\circ}$ siècle, il vient en échouer encore sept.

Nous ne commaissons que deux exemples dans le courant de (e siècle : le premier, en 1819, sur les côtes du IIolstein, 't un, en 182乡, sur les côtes de Rügen.

Il y a deux ans, à la suite d'une rupture de digue, on a trouve' près de Heyst une tête presque entière de Cachalot. Elle est déposée au Nusée de Bruxelles. On ne comait pas la date de la perte de cet animal.

Malm cite des exemples d'individus capturés sur les cotes de: Suède.

Linné én a cité sur les côtes de la Norwège.

Au nord de la Norwège, le professeur Sars cite le Cachalot parmi les espèces vues aux îles Loffoten (186\%).

On connaît aussi plusieurs exemples d'individus qui se sont. perdus dans la Baltique.

Le conseiller d'État Hensche a fait le relevé des Cachalots observés dans cette mer intérieure et de quelques Mysticètes dont on ne peut que deviner la nature spécifique 2.

Le plus ancien connu, un animal de 88 pieds de long, échoué en 1291 sur la côte de Weichtelmunde, a été conduit à Dantzig.

En 1364, un autre de 26 pieds, a été ígalement conduit ì Dantzig.

- Zelandia illustrata, midddlburgische courant, le 6 decembre $1784 \mathrm{et}$ Notulen, 4 arril, 1849.

2 Henscue, Schriflen d. Physit. Ak. gesells zu köniysberg, Juhrg, = I, Heft. II. 
Le 15 vetobre 14392 ou 1433 , un de 35 pieds a été pris vivant et conduit encore ì Dantzigg. Il avait été vu pendant 3 jour's. C'est sans doute une femelle.

Le 13 avril $14 \% 3$, un autre de 66 pieds de long fut jeté sur la côte, nähe dem Bulgaschen Tief.

En 1561, un jeune animal sous le nom de Baleine, long de 9 klafter, fut jeté sur la côte am alten Tief.

Le 19 septembre 1376 , un animal, décrit sous le nom de Physeter, de 14 pieds, se perdit encore dans cette mer; mais on peut se demander si c'est bien un Cachalot.

Plusieurs Cachalots sont allés mourir sur les côtes des lles Britanniques.

Les plus anciens ont été signalés par Sibbald : il parle d'abord d'une femelle qui a péri aux Orcades, en 1687; puis d'un màle de ${ }^{2} 2$ pieds, capturé, en février 1689, à Leine Kilns, au nord de l'embouchure du Forth. La màchoire portait 12 dents. Il fait mention également de toute une school, de 102 individus, qui a péri sur les côtes d'Écosse, mais, à en juger par la taille qu'il leur accorde, ce ne sont pas des Cachalots.

Un mâle de 322 pieds a échoué ensuite à l'embouchure du Forth vers la même époque; sa mandibule portait 48 dents.

Au siècle suivant, en $17 \% 6$, un autre mâle de $5 \%$ pieds est venu mourir également dans le Forth (côtes ouest de RossShire) et un en 1769 dont le sexe n'est pas indiqué.

James Robertson signale l'échouement d'un mâle, en 1770, également dans le Forth.

En 1762, un individu, long de 54 pieds, avec dix-huit dents de chaque côté, a été pris à Blythsand.

En 1769, on cite encore un màle de 54 pieds qui a péri au même endroit.

La même année, 1769, un Cachalot a été capturé sur la côte de Kent; vers la mème époque, un autre sur celle de Norfolk.

' H. Woon, Mag. of nat. hist, , 2e ser. t. III, 1829. 
En 182\%, un Cachalot mâle adulte est venu à la cóte à T'unstall, Jorkshire coast. Le squelette a 30 pieds de longueur.

Le 16 férrier 1829, un mâle de 62 pieds s'est perdu à l'entrée de la Tamise, à Whitstable, Kent. En attendant que les tribunaux eussent décidé qui était le propriétaire de cette épave, la marée a eu raison de ce cadarre et les os ont été dispersés.

Dans le courant du mois de mai de la mème année, un Cachalot fut aperçu en mer et bientôt capturé et remorqué dans la baie de Dunstafinage (Écosse). Il avait 60 pieds, mais le sexe n'est pas indiqué. La màchoire inférieure est conservée au château de Dunstaffnage.

On cite également un Cachalot qui a péri sur la côte de Curnouailles en juillet 1835 .

Dans la Faune des Oreades, par le Rév. Grorge Jow 1813, il est question de divers Cachalots qui ont visité cette ile.

Le dernier observé sur ces côtes est un animal de 60 a 70 pieds, en pleine décomposition, près de Thurso, au mois de juillet ou août 1863, dont le squelette est au British lluseum. C'est lui qui a servi à M. Flower pour la deseription des os 2.

Au mois de juillet 1871, un Cachalot mâle adulte a échoué à l'île de Shy. Son squelette est conservé à Édimbourg. La mandibule et le sternum sont très remarquables par l'âge de l'animal.

Le professeur Turner fait le relevé des Cachalots connus qui ont péri sur les côtes d'Écosse. Il en cite dix : un du XVII e siècle, quatre du XVIII siècle et cinq du XIX ${ }^{e}$ siècle. Sur huit individus, dont le sexe a été reconnu, il y avait cinq måles.

Le Cachalot étant un Cétacé équatorial, on a douté quelque-

- Aloensox, J., An account of a Whate of the spermaceli tribe, cast on shore on the Yorkstire coast, 1823. Trasict. Cunbr. phus. Suc., 1827. Fenussac, Bull. scien. nat., 1829. Isis, 1853, 1. 1006. Transzct. of the nat. histor. of Northumberland, vol. I, 1831.

"Report Brit. Assoc. for the advancement of science held at Newcastle, p. 106. 
fois de sa présence sur les còtes rlu Groënland. Les coites nord de l'Écosse ont été sourent considériés comme limite septentrionale de cet animal.

Nous avons le témoignage de plusieur's marins que ces animaux visitent parfois l'ouest et l'est du Groënland.

En 1718 et 1719 quelques Cachalots ont été pris, dit Zorgdrager, auprès du Groënland et auprès du cap Nord. Le plus remarquable avait 70 pieds environ et dans sa tête on a recueilli vingt-quatre tonnes de spermaceti 1 .

R. Brown ne fait mention que d'un seul Cachalot, capturé en 1857 près de Groven (720 L. N.)

Nous avons aussi le témoignage du gouverneur du Groënland: Le Cachalot est extrêmement rare sur la côte ouest du (iroënland, dit Holböll 2. Il n'a vu, pendant ses nombreux voyages, qu'une seule fois cet animal remarquable, et de souvenir d'homme, on n'en a vu échouer dans ees parages.

Les Esquimaux, qui ont visité Paris et Bruxelles en 18771878, araient des dents de Cachalots avec eux; ces dents étaient exhibées avec celles de Narval, de Horse et d'autres Delphinides.

A l'est du Groënland, apparait aussi parfois cet animal. Nous l'avons déjà dit plus haut, NI. Naugaret l'a rencontré aux environs de l'île Jan Meyen 3.

Il est fort curieux de comparer le nombre de captures ou plutôt d'échouements de Cachalots des siècles précédents avec. ceux des temps actuels.

Si l'on considère la rareté actuelle des Cachalots échonés et le nombre d'individus dont la capture est indiquée dans des livres anciens, on doit se dire que ces animax doivent être bien près d'être exterminés.

11 y a aussi quelques exemples de Cachalots eapturés ou

- Zorgdrager, Ed, ?.(18בð), p. 112.

- Escunicut, Nord. Malth, p. 195.

5 Revue des deux Mondes, 1er octobre 1869. 
échoués de l'autre côté de l'Atlantique, dans l'océan Pacificuee, dans la mer des Indes et dans les caux de nos antipodes.

Onze individus, la plupart jeunes, sont venus à la côte à l'est de la Floride, près du eap Canaveral, pendant l'hiver de 1882.

Le 29 mars 1842, un jeune animal de 16 pieds a été capturé près de Boston, New Bedford, U. S.

Le 3 décembre 1849 , on a trouvé la carcasse d'un mâle qu'on a remorquée au port Jachon; c'est le squelette de ret animal que M. Macleay a décrit.

Le corps d'une femelle échnuée a été remorqué à BotanyBay.

Sur les côtes de Tasmanie on a capturé un individu mâle, en 1861, dont le squelette se trouve au Collège royal des chirurgiens à Londres. On en connaît trois spécimens de Tasmanie dans ce remarquable Nusée.

Ce sont ces squelettes qui ont autorisé M. Flower à se prononcer sur l'identité du Cachalot d'Australie et celui d'Europe.

\section{MUSÉES.}

On a été longtemps sans connaître d'autre squelette de Cachalot que le squelette défectueux, dont nous avons parlé plus haut, et qui était monté à la cour du Muséum à Paris; Cuvier l'arait fait acheter à Londres, en 1818 1. Ce squelette, que personne ne regrette d'avoir vu disparaitre, sera bientôt remplacé par un autre.

Paris possède également une tête d'un animal adulte, prorvenant de la bande échouée à Audierne, en 1781, avec une colonne vertébrale, des mandibules et un os hyoïde.

Il se trouve également au Muséum à Paris des mandibules d'un mâle capturé à Bayonne. On y possède encore la têt. d'un jeune animal qui a péri à Rochefort.

- Daprès une note du memoire de M. Flower, ce squelelte est probablement le mème qui a figuré au Husée Backstrow, Flewt strcet, décrit dans ü ancien catalogue comme The Astonishing and complete Skeleton of a fuligrown Sperma-celi Whale. 
Mais, de toutes ces pièces, la plus remarquable est le squelette que le Muséum a reçu récemment par less soins du professeur Pouchet, et qui provient des îles Açores.

On conserve aujourd'hui, au Musie d Bayonne, le squelette d'un mâle qui a été capturé en $187 \%$.

A Londres, au Musée du College roval des chirurgiens, on possède de riches matériaux pour l'étude du Cachalot. Il s'y trouve deux squelettes, un jeune et un adulte, qui ont été 'nvoyés d'Australie. Cie sont ces pièces qui ont été figurées dans le beau mémoire du professeur Flower.

Le British Museum possède un autre squelette complet d'un animal capturé sur les côtes des lles Britanniques. Le British Museum possìde en outre la tête d'un mâle très adulte et qui $s$ 'y troure depuis fort Iongtemps; on ne comnait pas son origine, si je ne me trompe.

Pendant longtemps le seul squelette complet de Cachalot arlulte se trouvait à Burton-Constable Castle, près de Hull (Yorkshire). Il a été décrit par Beale.

A Cantorbéry, on conserve quelrues ossements d'un animal E'choué à l'embouchure de la Tamise. Pendant qu'on se disputait la propriété du corps, la marée dispersait les os du squelette.

Le Musée d'anatomie de l'Lniversité d'Édimbourgh possède la mandibule d'un jeune mâle, capturé dans l'Atlantique, à la latitude des Açores.

On possède en outre à Édimbourg le sternum et la mandibule d'un animal très adulte qui a échoué, en 1871, à l'île de Skey. Le sternum est énorme.

Edimbourg possède aussi une mandibule rapportée des îles Bauda (Archipel des Moluques), arril 1813, par des baleiniers, et sur laquelle se trouve un dessin du Cachalot que le professeur Turner a cru devoir reproduire.

Au Musée de Bologne on conserve un squelette qui a été obtenu par échange du Musée de Naples.

A Pise on conserve un squelette dans le vestibule du Jardin Botanique. 
On voit egalement une tête au Musée de Turin, mais dont nous ne connaissons pas l'origine.

On en possède des ossements à Pise, à Florence, à Bolognne, et à Ravenne, parmi lesquels il y a une portion de crâne.

Les ossements des divers individus qui sont venus échouer en $18 \% 3$ dans l'Adriatique, à Castel Nova, ont été pour la plupart conservés, mais ils ont été malheureusement mêlés: it Berlin, à Munich, à Trieste, on en conserve une tête; à Vienne un squelette, mais on n'a pas tenu compte ni du nombre de côtes ni du nombre de vertèbres, et il est fort douteux que les os de chaque squelette appartiennent au mème individu.

A Scheveningue (Hollande) on conserve une tête fort grandt dont nous avons parli plus haut dans une église située au pied des dunes.

Dans le cabinet de Camper se trouvaient: deux mandibules de 12 pieds et à vingt et une dents; une omoplate de 37 pouces de long et 26 pouces de haut avee une partie du membre (humérus, radius, cubitus) d'un animal de 63 pieds. Cl. Mulder pense que ces os proviennent de Cachalots capturés en 1762. - Ils sont actuellement conservés au Muséum dr. l'Académie de Groningue.

Le Musée de Bruxelles possède un atlas dort l'origine est inconnue. Il provient du cabinet du prince Charles de Lorraine. On y possède également une mandibule et une région cervicale, avec une côte mutilée, ainsi que la tête dont nous avons parlé plus haut. II n'est pas impossible que l'allas provienne d'un des Cachalots qui ont péri dans l'Escaut lr: 2 juillet 1377 , et dont Ambroise Paré a fait mention.

Au Musée de Sydney on voit un squelette qui a été décrit par Macleay en $185 \% 1$.

Au Muséum de Washington, on possède le squelette d'unc: femelle capturée sur la côte de New-Jersey et qui portait "u foetus d'un mètre environ de grandeur; on a monté l'animal. 


\section{DESSINS.}

Il y a peu de Cétacés aussi souvent reproduits par le dessin que le Cachalot, mais pendant lonģtemps on n'en a posséde: que de très défectueux. Aujourd'hui on en connait plusieurs bonnes tigures.

Tous les os du squelette sont également bien représentés.

Cet animal est même assez fidèlement reproduit sur des plaquettes en os par les pècheurs des côtes d'Alaska. Paul Gervais a figuré dans la Nature une plaquette d'os de Cachalof, sur laquelle on voit d'un côté un Cachalot, de l'autre côté une Baleine.

Le professeur sir Tumer a reproduit également un dessin de Cachalot, qui se trowve sur une mandibule de ce Cétacé, conservée au Musée d'anatomie d'Édimbourg.

Un conserve à la Bibliothèque royale de Bruxelles un certain nombre de gravures qui ont été réunies dans un portefeuille par Du Bus.

Ces gravures sont litites d'après des individus échoués sur les côtes de Belgique et de Hollande.

Le plus ancien est un Cachalot, capturé dans l'Escaut e'n juillet 1577 ; nous lisons sur la planche qui le reproduit : Ilet wataditig conterfeytsel en afmetinghe van desen walvisch, gevanyen den 2 july 1:575; ce dessin accompagne un manuscrit 736 ', Chromycli ran Inturemen, door Cuxknckex. Un texte explicatif, imprimé en flamand, latin, français, dit que la Baleine a été trouvée " tusschen Haeften gelegen in den Doel ende Saeftinghe in de Schelde, ontrent Antwerpen. ")

La Bibliothèque royale possède une autre gravure, représentant un Cachalot échoné, d'après l'inscription, la même année (1877) à Terheyden. C'est probablement le même que l. précédent.

Une autre gravure de la Bibliothèque royale représente un mâle, qui a péri, entre Katwyk et Scheveningen, en 10098. II avait 52 pieds de long. Nous en trouvons également un dessin 
dans Bor 1. Cette gravure ne parait ètre qu'une copie det porte pour inscription: Een walvisch lang 70 vocten, gestrandl op de hollumdsche zeetiust, tusschen Schereningen en Katwyl, in sprolikelmaend, 1598. Cet animal fut vendu publiquement pour 126 florins. Le rostre (Bek) fut donné au comte Jean de Nassau et envoyé ì Dillenburg. D'après Clusius, les grands os sont conservés à la Maison de ville de La IIaye.

Ambroise Paré reproduit le dessin d’un individu qui a péri dans l'Escaut 2; Clusius ćgalement en reproduit un qui est meilleur. Johnston en donne aussi une tigure 3.

Le professeur Sir 'Turner est en possession d'une gravare, représentant un Cachalot de 60 pieds de longueur, eapturé en 1601 dans le port d'Ancône 4.

Le sarant professeur de l'Tniversité d'Édimbourg a écrit une Notice intéressante à propos de cette gravure, qui n'est pas connue des naturalistes.

Sibbald a publié un dessin d'un mâle de "̈̌̉ pieds de long, qui a píri dans une baie du Forth en 1689. Ce dessin est loin d'être un modèle. Le dessinateur a représenté une tête de Baleine avee des évents au front. Ce eorps de 32 pieds, anguel il a mis un pénis, semblable à celui de la Balénoptère qui figure ì côté, pourrait bien être le corps de la femelle, échoué en 1687 , et non celui du mâle.

Le Cachalot, échoué en 161 4 près de Noorfuyli-op-Zée, a été reploduit par une gravure ronserve à la Bibliothèque royale; elle porte l'inscription suivante: 1 mon $161 \%$, den 28 december,

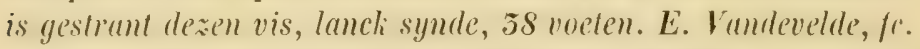

Une autre gravure représente igalement un mâle qui a péri, d’après l'inscription, à l'embouchure du fleuve Rodan, le 1'́ février 1619.

1 Bor, Nederl. histor. Amsterdam, in-fol., 1679, p. 433, 4" part.

2 Ambroise Pari, 25e livre de sés OEuvres.

s Ilistoria naluralis de Piscibus et Cetis, Amstelodami, 163̈7. Tab. XLII.

- Notes on som rare prinls of stranded Sperm-Whales. Jounx. of Axatomy ANo Puysiology, vol. XII, 1878. 
Nous y trouvons aussi une gravure d'un Cachalot de 62 pieds échoué en 1629.

Un autre, échoué en 1762 entre Zantroord et Wyk-aanZee, est figuré trois fois; des gravures en sont conservées à la Bibliothèque de Bruxelles. Nous lisons sur l'une : Cachelot gestrandt tusschen Zandroort en Wyk-op-Zee, 1762. J. Augustini, door H. Spilman, folio. Brux. Sur l'autre : Cagelot, lang (i1 voeten, den 20 febr. 1762 , tusschen Zantroort en Wyk-opZee aangedreven. Door Vander Vinne, $4^{\circ}$, mâle.

On trouve à la même Bibliothèque deux gravures représentant un Cachalot mảle de 60 pieds échoué vivant près de Egmond-op-Zee. Cagelot, Potwalvisch, Egmond-op-Zee, levend gestrandt den 13 febr. 1764, van 60 voeten.

La Bibliothèque royale de Bruxelles possède également un. belle gravure d'un Cachalot mâle de 60 pieds, qui a échoué à Beverwy.

On conserve, à Boulogne-sur-Mer, un dessin d'un Cachalot qui a péri près de la ville le $ّ$ mars 1761 .

Un mâle, dont nous avons parlé plus haut, est représenté dans les Act. nat. curios. Vol. III. (1733), Tab. I; il a péri à Villefranche, le 10 novembre 1716.

Le Cachalot mâle de 48 pieds, échoué le $1^{\text {er }}$ arril 1744 ou 1711 dans le golfe de Gascogne, a été dessiné également; Despelette, chirurgien-major de l'hôpital militaire de Bayonne, en a envoyé la figure à La Feyronie; c'est sans doute ce dessin qui est conservé dans la collection des vélins du Muséim. Fr. Cuvier l'a fait graver dans son Histoire naturelle des Cétacés. Nous en avons parlé plus haut.

Il existe deux autres dessins d'après le même animal, l'un' dans la Bibliothèque, l'autre dans les registres des délibérattions du conseil municipal de Bayonne.

Du Hamel 1 a publié aussi le dessin du mâle de 18 pierls,

1 Du Hamer, Hist. nat. des Péches, part. 2, sect. X, pl. XV.

TOME XLI. 
tué près de Bayonne en avril 1741. Il en a publié encore un autre dit Paul Gervais.

Tous ces dessins sont faits avec peu de soin et on peut dire, avec le $D^{r}$ Fischer, qu'ils sont fautifs.

Pennant fait figurer, dans sa Zoologie Britanniquee, un mảle de $\% 4$ pieds qui avait été pris à Bhythsand, en le considérant encore comme poisson $(1766)^{4}$.

Alderson représente l'animal entier, sa tête, son ail, etc. ?.

On trouve une copie d'un dessin de Valentin Cary à la Bibliothèque de Boulogne, fait d'après un Cachalot échoué le ö mars 1761, à 4 lieues de la ville.

Lacépède a figuré le Cachalot sous le nom de Physale cylindrique, Cachalot macrocéphale et Cachalot trompo.

Bomnaterre a figuré une femelle de la bande d'Audierne.

Le capitaine Scammon a publié un bon dessin de l'animal; on voit que le dos n'est pas pourvu d'une nageoire dorsale, mais de hosses, comme la Hégaptère. A côté du dessin il représente la coupe de l'animal qui est aplati du côté du dos, aminci du côté du ventre et arrondi sur les flancs.

Il représente sur une planche distincte l'animal nageant la bouche ouverte.

Du flamel représente une machoire de Cachalot, pl. IX, figg. 7 , et un mâle entier, pl. XV, fig. 3 .

Pierre Camper a reproduit la tête 3 .

Cuvier a fait graver la tête et plusieurs os dans ses Recherches 4 .

W. Wall a figuré le squelette complet en $185 \% 1$.

En 1868, le professeur Flower a publié un mémoire dans lequel se trouve une description complète de tous les os du squelette, accompagnée de sept belles planches.

2 Pensant, vol. III, pl. VI.

2 A few observation of the natural history of the Sperm Whale.

3 P. Camper, Cétacés, pl. XVIII, XIX et XXVII.

4 Ossem. foss., t. V, $1^{\text {re }}$ part., pl. XXIV. 
L.es principales parties du squelette sont figurées dans notre Ostéographie, pl. XVIII et pl. XIX, d'après les figures publiées par II. Flower.

Pander et d'Alton ont figuré une omoplate.

Le sternum du Cachalot adulte de l'île de Skye est figuré dans une notice de Turnex 1 .

Parmi les dessins, nous devons faire mention de celui de la tête d'un foetus, publić par R. Owen, dans la Description du rataloyue des squeletles du Musée royal du collège des chirurgiens 2 , et de la caisse tympanique 3.

Huxley a figuré le crâne d'un fœtus de Cachalot '4

Au Musée d'Edimbourg on voit sur une mandibule de Cachalot un dessin du même animal, fort bien exécuté.

On en connaît aussi des figurines en bois et en os taillés par les habitants des côtes d'Alaska et des Iles Aléoutiennes.

\section{COMHENSAUX ET PARASITES.}

Le Cachalot est encore bien incomplètement connu sous lt: rapport de ses commensaux et de ses parasites; Debell Bennet est le seul naturaliste que nous sachions, qui fasse mention de l'Otion Cuvierii trouvée sur sa lèvre inférieure et des Oniscus logés sur sa peau.

Il y est également fait mention de Cysticerques, trouvés dans de's kystes de la peau 5 , et Foster a trouvé des parasites qu'il a

1. Addilional Notes.... Proc. Roy. Soc. of Edinburgh. Session 1871-72, pi. 656-637.

2 Tome II, $\mathrm{n}^{\circ} 2417$.

${ }^{3}$ British fossi! Mammals, p. 520, fig. 20.

+ Manuel of the anatomy of vertebrated animals, p. 404, fig. 106, Londres, 1871.

S Debell Besset, Proc. Zool. Soc., 1857, p. õ0. 
envoyés à Sibbald, mais dont nous ne connaissons ni le nom ni la nature.

Sur les Cachalots, capturés en 1853, sur les côtes d'Istrie, on a trouvé des Balanes, mais on ne dit pas lesquelles '.

- Turver, Additional Noies on the Occurence of the Sperm-Wha'e, $1871-72$, p. 644 . 


\section{HYPEROODON ROSTRATUM.}

\section{LITTÉRATURE.}

whas Tallor, II istory and antiquities of IIarwich, and Dover-court wilh notes and observalions relating to natural history. London, 1750, in- $4^{\circ}$.

w. Nontoppidan, The nalural history of Norway. London, 1755.

Chemnitz, Beschäftigungen der Berlinischen Gesellschuft Waturforcher, $1773 \cdot 177 !$ ! t. IV, 1779.

Joln Inuter, Observalions on the structure and ceconomy of Whales. Pullosophical transaction, vol. LXXVII, 1787.

Laussard, Mémoire sur deux Cétacés échoués vers Honfeur, le $19 \mathrm{sep}$. tembre 1788; dans Rozıen, Obs. surla physique, sur l'histoire ualurelle..., t. XXXIV. Paris, 1789.

Iacépète, Iisloire naturelle des Célacés. Paris, an XII de la Rẻpublique, 1804.

Cuvier, Recherches sur les ossements fossiles. Paris.

C. Dosmael, Notice zoologique sur un IIyperoodon. Nouveaux Mésoires de L'ACadéuie royale des sciences et belles-lettres de Bauxelles, t. XII, 1841.

Eudes de Longehamps, Remarques zoologiques el analomiques su० i'llyperoodon. Mémotres de la Société linnéende de Normande, vol. VII. Paris, 1842. 
Dellingham, o. B. Short description of a Bollle-nose Whate. (IIyperoodon Butzkopf), Ann. of nat hist., $181 \bar{j}$.

Gray, Zoology of the voyage of Eribus and Terror, 1844.

vrolik, Natuur en ontlecdlundige Beschouwing van den IIyperoodon. Haarlem. 1848.

Eschricht, Untersuchungen üher dic nordischen Wallhiere. Leipzig, 1849.

Duvernoy, Célacés vivants et fossilcs. Ann. sc, nalur., 5e sér., t. XV, p. 44, 1851 .

John strutuers, Account of rudimentary finger muscles found in a toolhed Whale (Hyperoodon bidens). Journ. of anatomy and pliysiology, novembre 1875.

Thom. Southwell, on a Sliull of Hypcrondon latifrons. The Zoologist, 1881, p. 258.

Fischer, Cítacés du sud-ouest de la France, 1881.

Flower, On the Cranium of a new spccies of Hyperoodon... Proc. Zoor. Soc., may 1882.

Flower, On the whales of the genus IJyperoodon. Proc. Zoov. Soc., déc. 1882.

Thom. southwell, $\mathrm{O}_{n}$ the beaked or bottle-nose whale (Hyperoodon). Trans. norf. and noriv. naturalists' Soc, vol III, 1882.

bavid Gray, Noles on the characters and habils of the Bolllenose Whalc (Hyperoodon rostratus). Proc. Zool. Soc., déc. 1872, n०726.

nr A. Cerstiiclcer, Das Skclet des Döglings, Iypcroodon ros/ralus, Pout. Lcipzig, 1887. 


\section{HISTORIQUE.}

Les pêcheurs de Färö̈r et d'Islande ont connu l'Iyperoodon longtemps arant les naturalistes. Ils le désignaient sous le nom de Dögling.

Au commencement du siècle précédent, deux Hyperoodons échouent en Angleterre sur les còtes d'Essex; il en est fait mention dans une Histoire des antiquités de IJarwich, avec des notes sur l'histoire naturelle, par Dale. L'un, de 1/4 pieds, se perdit en 1717 près de Malden; l'autre, de 21 pieds, près de Bradwel, à peu près en même temps que le précédent.

En 17:30, E. Pontoppidan publie une histoire naturelle de Norwège et il décrit l'Hyperoodon sous le nom de Balcena rostrata, nom spécifique qui a produit pendant longtemps une grande confusion. Fabricius a donné le même nom à la petite espèce de Balénoptère.

Chemnitz fait mention, en 1779 , sous le même nom de Balcena rostrata, d'un mâle, capturé dans les eaux de Spitzberg, dont les principales parties du corps ont été apportées à Copenhague.

En 1783, un Hyperoodon est capturé dans la Tamise, près de London-Bridge; il tombe heureusement dans les mains de Hunter, qui, tout en ne connaissant pas bien l'animal, n'en a pas moins parfaitement exposé son organisation.

Ce travail n'était pas seulement classique pour l'époque où il a paru, dit Eschricht, mais c'est la collection de faits anatomiques la plus remarquable sur les Cétacés; on y trouve tout ce que l'on sait sur les Hyperoodons, dit-il. Même le dessin de Ilunter est un des meilleurs qui existent de cet animal, dit le savant professeur danois.

Deux années après la publication de Hunter, Baussard, un officier de marine, publia des observations sur deux femelles, l'une de $231 / 2$ pieds, l'autre de $12 \%$, qui venaient d'échouer à Honfleur, à l'embouchure de la seine. La description de la 
peau qui tapisse lo palais, a fait croire à Lacépède que ces animaux portaient des dents au palais; c'est l'origine du mot ilyperoodon, qui est accepté aujourd'hui par tous les cétologistes.

L'Hyperoodon n'était pas encore représenté par son squelette au Muséum de Paris, à l'époque où Cuvier écrivait ses Recherches sur les ossements fossiles et la description qu'il en donne est faite d'après le squelette de Hunter.

En 1811, Wesmael publia une notice zoologique sur un Hyperoodon, à propos d'un animal échoué le 16 septembre 1810 sur un banc de sable près de Ziericzee. M. Wesinael cherche à mettre d'accord les divers auteur's qui se sont occupés de cet animal. On a fait avant lui presque autant d'espèces qu'il est échoué d'individus, dit-il avec raison, et il exprime l'opinion, qu'il n'y a qu'une espèce d'Ilyperoodon, à laquelle il conserve le nom spécifique de rostratum, proposé par Pontoppidan.

En $18 \%$, le Dr A. Jacob a publié à Dublin le résultat de ses observations anatomiques et zoologiques sur ce même animal.

A l'oceasion d'un IIyperoodon échoué en 1816 à Lantvoord, W. Vrolik publie, en 1848, un mémoire fort intéressant sur ce Cétacé et ne néglige pas d'étudier l'animal sous le rapport anatomique. Le sarant anatomiste d'Amsterdam passe en revue lappareil respiratoire, digestif, circulatoire, sexuel femelle, l'œil, la peau, et décrit avec soin le squelette sans négliger les os du bassin. Quinze belles planches accompagnent ce mémoire.

Il parle avec raison du temps, de l'argent et des efforts extraordinaires que l'on doit faire pour obtenir un mince résultat dans l'étude d'un Cétacé.

En 1849, Eschricht fait paraitre ses Nordischen Wullthicre, et consacre un chapitre à l'histoire du Cétacé qui nous occupe. Nous lisons en tête de ce chapitre : Ueber die Schnabelwalle "(Rhynchoceti, die Zahnlosen Delphine, Schlegels, Iyperoo" dontina, Gray's) im algemeinen und den Entenwall (Chonocetus "rostralus, Iyperodon rostratum, auctorum im besondern." 
II expose $: 1^{\circ}$ ce qui est connu de ces animaux; $2^{\circ}$ leur répartition aux hautes latitudes et la connaissance que les habitants de ces régions avaient d'eux; $3^{\circ}$ leurs caractères extérieurs; $4^{0}$ leur dentition; $5^{\circ}$ leur cavité de la bouche; $6^{\circ}$ leur estomac; $7^{\circ}$ leur muqueuse intestinale; $8^{\circ}$ leur ostéologie; 9` leur place systématique dans l'ordre des Cétacés; $10^{\circ}$ la comparaison de ces faits avec les observations des auteurs les plus récents.

Les Rhynchoceti ne comprennent que deux genres: Chanocetus ou IIyperoodon, et Micropteron.

On a beaucoup écrit sur les Hyperoodons depuis Eschricht, mais, à l'exception des observations sur la différence des sexes qu'Eschricht soupsonnait, nous ne trouvons rien de bien important à ajouter. On a été, jusque dans ces derniers temps, dans le doute sur l'existence d'une seconde ou de plusieurs espèces de ce genre dans nos mers. On avait pêché un Hyperoodon avec des maxillaires très élevés au milieu du rostre et se j ignant même sur la ligne médiane; Gray lui avait donné le nom d'Ilyperoodon latifrons. Eschricht avait émis l'opinion que cette forme extraordinaire pouvait bien indiquer le mâle; les individus capturés dont on avait constaté le sexe étaient à peu près tous femelles. Eschricht avait raison, comme nous le verrons plus loin.

Ln médecin islandais, Haalland, a envoyé à Eschricht ' les principales parties du corps d'un Hyperoodon conservé dans le sel et dans l'alcool, et lui a fait part de plusieurs observations intéressantes faites sur deux individus, dont un fut eapturé.

Eschricht a reconnu que, dans la description qu'il avait donnée de l'estomac, il avait été induit en erreur par la mauvaise conservation de la pièce qu'il avait eue d'abord sous les yeux.

Dans le fotus, l'estomac présente déjà tous les caractères de l'adulte.

Le professeur Struthers (1871) a publié le résultat de ses observations sur les muscles des doigts d'un Hyperoodon échoué sur les côtes d'Écosse.

1 Escuricit, Nord. Walle., p. 26. 
En 1882, M. W. Flower 1 a fait connaître un cràne d'Hyperoodon, trouvé au nord-ouest de l'Australie, à l'île Lewis. Quoique mutilé, il appartient évidemment à ce genre, mais il présente des caractères assez particulier's pour ne pas le confondre avec l'espèce de notre hémisphère, dit M. Flower, qui le désigne sous le nom d'IIyperoodon planifrons.

A la séance du 19 décembre de cette même année, $\mathbf{M}$. W. Flower - communiqua une note dans laquelle il fit l'historique de l'IIyperoodon latifrons proposé par Gray, et fit connaître les observations de David Gray, commandant du baleinier l'Éclipse, d'après lequel le prétendu IIyperoodon latifrons est tout simplement, comme Eschricht l'avait soupçonné depuis trente ans, un Hyperoodon ordinaire du sexe mâle.

Le capitaine de l'Éclipse fait connaître en même temps les faits les plus intéressants sur le séjour de ce Cétacé à l'est du Groenland.

Au mois d'avril 1884, M. Southwell, de son côté, a publié des faits d'une grande importance sur la pèche des Hyperoodons.

Le Docteur-professeur Gerstäcker vient de publier une monographie du squelette des Cétacés, traitant surtout de l'IIyperoodon rostratus. Au commencement du mois de février 1877, un individu est venu se perdre la nuit sur un banc de sable sur les côtes de la Baltique.

II. Gerstäcker étudie d’abord la tête et s'étend longuement sur l'os lacrymal, que P. Gervais a bien décrit, dit-il ; il étudie ensuite la colonne vertébrale et consacre un chapitre à la région cervicale, un autre aux vertèbres dorsales, aux cötes et au sternum. A propos des os lacrymaux, il fait remarquer que, contrairement à l'avis de Stannius et de Flower, ces os existent dans les genres Delphinus, Phocena, Lagenorhynchus,

1 Flower, On the Cranium of a new species of Hypcroodon..., Proc. Zool. Soc., Loudon, 1882.

- Flower, On the Whales of the genus Hyperoodon, Proc. Zool. Soc, déc. 1882. 
Tursiops et Globiceps, comme dans les Hyperoodons. II s'étend ensuite sur les rapports morphologiques des côtes et des apophyses transverses des vertèbres dorsales.

Après avoir passé en revue les autres parties du squelette el fait remarquer le peu de développement des nageoires pectorales, si bien exposées par Vrolik, il compare la colonne vertébrale des Cétacés à la colonne vertébrale des autres ordres de mammifères.

Les figures qui accompagnent ce travail montrent fort bien la différence fondamentale qui existe dès l'origine entre les diapophyses et les parapophyses. Les dernières dorsales des Hyperoodons sont, sous ce rapport, très instructives.

\section{SYNONYMIE.}

Delphimus diodon. Hunter.

Hyperoodon diodon. Lacépède.

- rostratum, Chemnitz.

- latifrons, Gray.

Butzkopf, Entenwall, des Allemands.

Dügling, des habitants de Färoër.

Bottlenose Whale, des Anglais.

Andvhalar ou Andarnefia, des Islandais.

Anarnak, des Groenlandais.

Le Dauphin diodon de Lacépède est l'IIyperoodon; on lui a donné successivement les noms de Delphimus diodon, d'Edentulus, de Monorlon, d'Ancylodon, d'Aodon, d'Hétérodon et d'Iyperdon; celui qui lui est conservé, Iyperoodon, repose, comme nous l'avons dit plus haut, sur une erreur d'observation.

L'Hyperoodon latifrons est bien, comme Eschricht l'avait montré, basé sur un crâne de mâle : il avait trouvé déjì des différences sexuelles semblables dans d'autres Cútacés, parmi lesquels il cite le Gilobiceps. 
L'Hyperoodon semijunctus de Cope est un Ziphius cavirostris 1.

\section{CARACTĖRES ET DESCRIPTION.}

$\Lambda$ prìs le Cachalot, c’est la plus grande espèce de Ziphioïde. On la reconnaît facilement ì ses petites nageoires pectorales et à l'absence presque totale de dents aux deux mâchoires. Nous disons, absence, parce que les deux, ou quelquefois les quatre dents du bout du maxillaire inférieur, ne percent que faiblement les gencives.

On ne peut confondre l'Hyperoodon avec aucun autre Cétacé; il a la tête tronquée comme le Cachalot, les dents manquent complètement à la mâchoire supérieure, et, à la mâchoire inférieure, on en trouve, tout au bout antérieur, une ou deux paires assez petites. La tête osseuse a surtout de remarquable, que les maxillaires s'élèvent verticalement comme deux ailes qui convergent l'une vers l'autre sur la ligne médiane, et qui, chez les mâles, se touchent au milieu du rostre.

Ils ont deux sillons sous la gorge. Ces sillons sont bien représentés par Gray, planche III, d'Erebus and Terror ; Vrolik les représente aussi dans son dessin.

Blainville les a signalé, sous le menton du Micropteron.

Le capitaine Scammon a reconnu ces mêmes sillons, même plus prononcés, dans le Rhuchianectes glaucus, du Pacitique.

La couleur de la peau varie du noir au jaune pâle, chez les jeunes, au jaune brun, chez les adultes; le rostre et le front deviennent blanc avec l'âge, et on voit apparaitre une bande blanche autour du cou.

La coloration est, comme dans les autres cétacés, plus claire en dessous qu'en dessus.

La graisse renferme, comme celle du Cachalot, une certaine quantité de spermaceti ou blane de Baleine?

- Fied. True, A note upun the Hyperoodon semijunctus of Cope. Proc.

Ux. Sr. nat. MUSEum, 1885.

- Elle a plus de valeur comnerciale que celle des autres Cétacés. 
La coionne vertébrale n'a que quarante-cinq vertèbres, sept cervicales, neuf dorsales, dix lombaires et dix-neuf caudales.

Les cervicales sont réunies comme dans les vraies Baleines.

L'articulation de la sixième côte est fort intéressante; elle sarticule encore supérieurement, à l'arc neural et à l'apophyse transverse, tandis que la septième ne s'articule plus qu'avec l'apophyse transverse, comme les suivantes.

On compte, dans la nageoire pectorale, une phalange au pouce, cing à l'index, cinq au médian, quatre à l'annulaire et deux au petit doigt.

l'estomac est formé de sept poches qui se suivent; il y en à encore plus dans le Mésoplodon. Je trouve dans mes notes que cet estomac existe déjà a vec les mêmes caractères dans le foutus. Eschricht doutait encore s'il existe une communication directe entre ces chambres; je la lui. ai montrée à l'aide d'un stylet sur un foetus pendant mon séjour à Copenhague en 1856.

Le professeur John Struthers a fait connaitre les muscles des nageoires pectorales à la section de Zoologie de l'Association britannique à Aberdeen.

L'Hyperoodon a au moins 6 pieds en venant au monde; les jeunes qu'on a trouvés en compagnie de leur mère avaient de 8 à 16 pieds. La femelle atteint de 27 à 28 pieds. On ne sait si le mâle devient plus grand.

La taille des individus capturés en Europe varie entre 8 et $28 \mathrm{1} / 2$ pieds: les individus de 8 à 16 pieds suiraient encore leur mère.

David Gray accorde 30 pieds à l'adulte et 10 pieds au jeune en naissant. Ce dernier chiffre est exagéré.

On a donné la mesure de plusieurs mâles qui ont été capturés : un à Belfast qui avait 20 pieds, un autre décrit par Dale en avait 21; celui décrit par Chemnitz en avait 23 ; le plus grand est celui de Dunkerque, qui mesurait entre 27 et 28 pieds.

On a capturé à peu près autant de femelles; leur taille varie de 14 à 28 pieds et demi. La femelle décrite par Dale (1717) 
n'en avait que 14; celle de Porstoppidan, qui était pleine, portait un foetus de 6 pieds; les femelles de Honfleur (1788), de Baussard, de Portland, de Zandroort, avaient de 23 à 24 pieds; une seule, celle de Sèignasse, en avait 28 et demi.

Il n'y a pas d'observation faisant voir que les mâles sont plus grands que les femelles, dit Eschricht.

\section{MOEURS.}

L'Hyperoodon vit par petites bandes; lorsqu'on a commencé à le poursuirre, il était confiant d'abord, mais il est devenu craintif et méfiant.

Aujourd'hui on a quelque peine à l'approcher. Le moindre bruit le met en fuite. Les pêcheurs des Fürö̈r savent que, pour atteindre l'animal, ils doivent placer leurs gants de laine entre la rame et le canot sur lequel elle s'appuie.

Si l'on en juge par les individus qui sont venus se perdre sur les côtes et qui sont ordinairement deux, il y a lieu de croire que les Hyperoodons sont monogames.

On voit parfois aussi un jeune màle avec une femelle et un vieux mâle.

On observe dans les eaux de Spitzberg que les Hyperoodons fréquentent la mer ouverte le long des glaces, en petites gammes de 4 à 10 individus, ayant un vieux mâle comme leader.

La pâture des Hyperoodons consiste principalement en Céphalopodes. On connaît le contenu de l'estomac de l'animal ouvert par Hunter, par Baussard, par Vrolik, et ce dernier estime le nombre de bees trouvés dans un seul animal à dix mille; il y en a parfois non seulement dans l'estomac, mais aussi dans le commencement de l'intestin. La fin de l'intestin n'en renferme pas. En 1841 Eschricht a signalé, d'après Haalland, dans leur estomac, des becs de Céphalopodes qu'il rapporte à l'Onichoteuthis Fabricii, à une Sépia, à un Loligo, des restes de poisson et une Holothurie. 
Ils trouvent ces nombreux mollusques surtout dans les grandes profondeurs.

L'estomac d'une mère capturée en septembre 1788 à l'embouchure de la Seine ne contenait que des becs de Céphalopodes; le jeune n'avait dans son estomac que de l'eau blanchâtre.

L'individu de 24 pieds, capturé en septembre 1880 près de Bangor, ainsi que la femelle capturée en novembre 1880 sur la côte de Kent, avaient également l'estomac plein de bees de Céphalopodes.

On n'a trouvé dans l'estomac d'un autre individu capturé que des morceaux de bois et des cailloux de la grosseur d'une grosse poire.

Des baleiniers prétendent que cet animal peut rester deux heures sous l'eau.

Il beugle d'une manière effrayante s'il échoue.

On a sourent parlé de l'odeur infecte du cadarre des Cétacés en général. Eschricht m'écrivait en mars 1861 : je ne conçois pas comment tous les observateur's se plaignent de l'odeur infecte d'un cadavre de Cétacé. Celui-ci (un Hyperoodon) était mort depuis trois mois; mais exviscéré, il ne puait pas beaucoup. Il est vrai que c'était en plein hiver.

On prétend que le lard de ce Cétacé est purgatif '.

\section{DISTRIBUTION HYDROGRAPHIQUE.}

L'Hyperoodon semble se tenix pendant l'été dans les mers arctiques, où il vit par petites bandes, et en haute mer. En automne il se rend au sud en passant, ou sur les côtes d'Islande, ou sur les côtes de Färoër où il arrive à peu près à époque fixe. Dans cette dernière île, il est même l'objet d'une pèche régulière; on en prend tous les ans vers la fin d'octobre cinq ou six, et chose curieuse, ce sont toujours des femelles.

3 Malagren, Troschel's Archiv., 1861, p. 92. 
En 1886, le commandant de l'Éclipse part pour la péche dans la Mer de Baffin; il quitte Peterhead (Écosse) le 20 avril et à cent milles des cùtes il rencontre plusieurs Schools d'Hyperoodons; quatre jours après il en rencontre encore et entre autres un Orque, très reconnaissable, dit-il, à sa nageoire dorsale, du moins le mâle. Le 26 , le 27 , il voit de nouveau l'Hyperoodon, et il apperçoit le même Cétacé jusqu'au dernier jour en approchant des glaces 1. Ces Hyperoodons se rendent sans doute ì cette époque de l'année dans leur quartier d'été, comme plusieurs autres Cétacés.

On peut considérer les profondeurs de l'Atlantique septentrionale, de janvier à mars, comme station d'hiver des Hyperoodons, dit Eschricht. Cependant ils seraient très rares sur les côtes ouest d'Islande, dit Haalland, et, d'après Holböll, ils le seraient également sur la côte ouest de Groënland. Ils sont sans doute rares à la latitude à laquelle Holböll avait sa résidence, mais, à l'époque où Holböll gouvernait ce pays, les baleiniers ne croyaient pas les Hyperoodons assez importants pour leur faire la chasse; ils étaient confondus avee les autres espèces qui ne donnent aucun profit.

On voit les Hyperoodons dans le détroit de Davis, jusquau 7(1) degré latitude nord, dans le détroit de Hudson, et dans la baie de Cumberland.

On le voit aussi autour du cap Farewell, autour de l'Islande, de Jan Meyen, jusqu'au 7 $7^{\text {e }}$ degré autour de Beereneiland, e't jusqu'à la Nouvelle-Zemble.

Ils fréquentent la mer ouverte dans ces régions, le long des glaces, en petites gammes de 4 a 10 individus.

Fréd. Martens cite l'Hyperoodon, sous le nom de Butskop, parmi les Cétacés observés au Spitzberg.

Chemnitz, de mème, a reconnu les Hyperoodons à la hauteur de Spitzberg à la fin de l'été en 1777.

Malmgren a fait l'observation que l'Hyperoodon ne se trouve plus dans les eaux dont la temperature descend au-dessous

1 Roв. Gray, The Zoolugist, fér. 1887. 
de $-3^{\circ}$; il en a vu en allant et en revenant du Spitzberg, mais seulement dans les eaux d'azur chauffées par le Gulfstream.

A son retour du Spitzberg, il en a vu reparaitre le 14 septembre sous la latitude de $78^{\circ}$ nord.

Nous connaissons quelques exemples d'Hypéroodons perdus sur les côtes de Danemark, de Norvège et de Suède. Pontoppidan cite deux échouements sur les côtes de Norvège; nous en avons parlé plus haut.

Le 14 novembre 1838, quatre individus périssent sur les mèmes côtes, deux vieux, l'un de 24, l'autre de 27 pieds, et deux jeunes, chacun de 8 pieds.

Dans le courant du mois d'août 1846, dans l'intervalle de quelques jours, il en vient deux à la côte dans la baie de Christiania, près de Frederichstadt.

Vers la fin du mois d'octobre 1860 , une petite school de cinq individus est venue se perdre sur la côte de Jutland (Danemark), après un très gros temps. Parmi eux se trouvait un nouveau-né qui a été malheureusement massacré, m’écrivait Eschricht. Je me suis rendu sur les lieux, dit-il dans une lettre du 28 novembre 1860, mais il était trop tard pour étudier le nouveau-né, les débris en étaient dispersés !

En avril 1881, un Hypéroodon mâle a échoué aux îles Loffoden; son crâne est conservé au Musée de Christiania.

En draguant dans la mer du Nord, sur le great Fischer Bank, on a recueilli la tête d'un mâle adulte. Elle est déposée à Norwich. Nous avons fait connaître une tête de Balcenoptera rostrata recueillie dans les mêmes conditions, également dans la mer du Nord.

${ }^{2}$ Yoici comment Eschricht s'exprimait à ce sujet en français:

“L'orage qui a jeté au mois d'octobre une masse de navires sur nos côtes parait maintenant teteud:e ses effets aux Hyperoodons. II n'y en a pas moins de cinq qui viennt nt d'échouer sur nos côtes. Ce qui m’a fait le plus de peine, c'est qu'un nouveau-né se Irourait parmi eux et qu'il a été complèlement massacré par ces .... Je viens de donner ordre qu'on m'envoie les viscères qui sont enterrés ». (Leltre du 9 mars 1861.)

Tome XLI. 
L'Hypéroodon pénètre parfois dans la Baltique. Le $\therefore$ lécembre 1801 , un individu de sexe femelle se fait capturer dans la baie de Kiel. Le squelette est conservé au Musće de l'Ecole vétérinaire de Copenhague. L'animal a été figuré dans Voigt!.

Ln autre individu a échoué en 1807 sur la côte de Holstein. Il a été acheté par le professeur Viborg de Kiel.

En avril 1823, un IIyperoodon s'est perdu près de Landskiona (Oresund) dit Nilsson ${ }^{2}$.

Dans la nuit du 11 férrier 1577, un Hypéroodon est venu se perdre sur un banc de sable au fond de la Baltique (presqu ile der Zingst, 30" $40^{\prime}$ long. "37 $28^{\prime}$ lat.). Les os sont conservés it Cireifswald. Le professeur Möbius fait mention de cette capture.

Un autre est venu à la côte à l'ouest de Rügen, en IS8....

le's côtes des Iles Britamiques nous en fommissent aussi plusieurs exemples :

Ireux Hỵpéroodons sont capturís sur les côtes d'Angleterre, au commencement du siècle dernier; Dale, comme nous larons dit plus haut, en fait mention dans un appendice de la Topographie de Harwich en 1730 et Dover-court; l'un était du sexe femelle, dit Dale; il avait 11 pieds; il a été capturé les weptembre 1717. Lautre, pris à quelques jours de distance, avait 21 pieds. Dale croit le clernier un mâle.

Jacob et William Thompson ont connu l'échonement de seizs: Hypéroodons, dont neuf sur les côtes d'Trlande, deux sur les côtes d'Angleterre, un dans le Firth of Forth.

En 1783, un animal de 21 pieds fut capturé dans la Tamise.

On fait mention de plusicurs individus trouvés isolés silr les cotes d'Angleterre, dont un sur les côtes d'Essex 23.3 segtembre 1817;.

On cite un animal de 16 pieds, un jeune par conséquent, qui a été capturé au printemps de 1829 près de Dublin et désigné sous le nom de Delphinus diodon ${ }^{5}$.

I Fréd. Cuvier, Histoire nalurelle des Cétacés, p. 24.

2 Nills Nilsson, Sliandinanisk Fauna, Lund, 188 əั.

5 R.-J. Gruves, Trans, roy, irish. Acad. Dublin, 182อั. Isis, 1828, [. 31. 
Lne vieille femelle de 2 't pieds et une jeune de 16 pieds \& pouces ont échoué au commencement du mois d'octobre (1848?), in Portland Roads.

Dans la baie de Belfast, un Iypéroodon a été capturé en septembre 1857 '.

Lne mère et son jeune ont été eapturés le 18 novembre 1860 ¿t l'embonchure de la Tamise, à Whitstable (Kent) . Ils avaient une grande quantité de becs de Poulpes dans l'estomac.

On a vu un individu échouer vivant, à Fraserburg, Aberdeenshire coast, le 17 aouit 1871. II arait 20 pieds de longueur (Struthers).

Le 16 septembre, dans la baie de Ballyholn, près de Bangor, ın individu de 24 pieds est venu à la côte.

A l'Association britannique d'Édimbours, le professeur struthers a fait mention d'un mâle de 20 pieds, venu à la côte, et qu'il a eu l'occasion de disséquer.

En jeune mâle a été capturé en novembre $188 \%$ à Dunbar, cótes d'Ecosse. Il avait des dents rudimentaires au maxillaire supérieur et inférieur ${ }^{5}$.

Plusicurs échouements ont 'u lieu sur les côtes de Belggique et des Pays-Bas : en 1783, un individu, décrit par Pierre Camper, dont le crâne est conservé à Saardam, est venu se perdre sur les côtes de Hollande.

En septembre 18\%0, un individu a échoué près de Ziericzer; il a été décrit et figuré par Wesmael.

Ln autre, de sexe fenchle, comme le précédent, est venu à lil côte à Zantroort, le 24 juillet 18 16 . Il a eté décrit par Vrolik.

Un autre est venu se perdre dans l'Escaut, en 1873; son squelette est conservé à Liège.

Lne femelle de $7^{m, 90}$ est allé mourir à l'île Texel, le 13 novembre 188 .

- Proceed. Dubiin univers. Zuol. and Bol. Association, vol. 1, part. 1, p. 4.

2 Procred. Zool. Soc , 1860, p. 575 -Illustrated neu s, 18 novembre 1860.

s Prof. Tlrakr. Royal physical Socirly, January 20,1886. 
Plusieurs Hypéroodons sont venus échouer sur les côtes océaniques de France.

On y a constaté huit fois des échouements d'Hypéroodon, dit le $\mathrm{D}^{\mathrm{r}}$ Fischer en 1881.

On en cite un exemple au siècle dernier. Le 19 septembre 1788, une mère et son petit furent pris à Honfleur, à l'embouchure de la Seine; Ie petit avait 12 pieds, la mère, 23. L'estomac du petit ne contenait que de l'eau blanchâtre; la mère avait des bees de Céphalopodes dans le sien '.

Un autre a péri, le 12 octobre 1810, sur les côtes de la Gironde (Bassin d'Arcachon) 2; c'est le point le plus méridional à l'est de l'Atlantique, visité par cette espèce.

Sur la plage de Lagraner, près de Caen, il en est échoué un autre encore en 1840.

Eudes Deslongchamps fait connaître la perte d'un animal sur la côte de Normandie, en 1812. Son squelette est à Paris.

Vers le 17 ou le 18 décembre 1879, une femelle est venue échouer vivante à Hillian (Côtes-du-Nord), dans le voisinage de Saint-Brieuc. Le squelette est au Musée de l'Lniversité de Liège. Il est fort incomplet. L'animal se débattait en beuglant, disaient les habitants de la côte qui avaient entendu un bruit insolite pendant la nuit.

Le 26 septembre 1880, une femelle, accompagnée de son jeune, a été capturée à Aigues-Mortes (Gard) et une partie du squelette avec le crâne du jeune sont au Muséum à Paris; ils moururent, peu de temps après leur prise; la mère, poussait des mugissements, disent les témoins de cette scène 3 .

Le 25 juin 1884, une femelle de $9^{\mathrm{m}}, \mathrm{30}$ a échoué à Seignasse, près du cap Breton. Le squelette est au Muséum à Paris.

- Bauss.ind. Mémoire sur deux Célacés échoués vers Ilonfleur, Jourial de PHYSIQUE, 1789, P. 201.

${ }^{2}$ Bull. polym. du Muséum d'instruction publ. de Bordeaux, décembre 1810. Fréd. Cuvier, Hist. nal. Cét, p. 217. Barguet, Act. Soc.linn., Bordeaux, 1. XIII, $184 \overline{3}$.

${ }^{3}$ Clément, Bulletin Soc. d'élud'e des Sic. nal. de Nîmes, janvier 1881. 
Depuis lors, MII. Pouchet et Beauregard ont fait connaitre un Hypéroodon, échoué à Rosendael, près de Dunkerque, le 24 juillet $188 \%$. II portait des harpons dans les flancs. Le cadarre a été acheté pour l'huile, et le squelette en est perdu.

Le 19 août 1886, quatre Hypéroodons ont fait leur apparition à Saint-Vaast-la-Hougue. Deux ont été capturés. Tous les deux sont femelles. Lne d'elles a expulsé un foetus pendant qu'elle était étendue sur la grève. Il est conservé.

Jusqu'à présent on n'a vu qu'un Hypéroodon pénétrer dans la Méditerranée. Il existe, il est vrai, un squelette au Musée d'Alexandrie, mais il est possible qu'il provienne de l'Atlantique.

L'Hypéroodon de F. Cuvier 1, échoué sur les côtes de Toscane, en 1830, s'il a :00 pieds de long, n'est évidemment pas un Hypéroodon, c’est plutôt un Cachalot. Le seul Hypéroodon connu de la Méditerranée est celui d'Aigues-Mortes.

Cet animal est également connu de l'autre côté de l'Atlantique :

On en a vu venir à la côte en hiver (janvier 1869) à l'est des Etats-Lnis d'Amérique (à Nort Dennis) et II. Allen, fait remarquer que les Hypéroodons arrivent parfois par bandes, en automne, dans les baies de Massachusets 2 .

Reinhardt fait observer qu'un Hypéroodon a échoué sur la côte des Etats-Lnis d'Amérique, sous le nom de Iesoplodon Sowerbyr.

On a capturé un Hypirroodon dans le port de Newport (EtatsLnis), qui mesurait 27 pieds. Ln second a échappé. Les os sont conservés.

Un autre a eté pris au cap Cod, de 2 '́ pieds de longueur.

II. Cope reproduit le dessin d'un individu capturé sur la cóte de Rhode Island.

En faisant le relevé de la date à laquelle des individus ont échoué, nous n'en trouvons qu'un en été (2) juillet, IIollande); quatre en septenbre Essex, Belfast, Escaut et embouchure

2. Cuvien, Cél., p. 586.

2 Sometimes come into ourbay in large schools. Allen. Mamm. Massachus. 
de la Seine); deux en octobre (Danemark et Portland road: un en octobre (Arcachon); un en novembre (dans l'Escaut, et le même mois 1838, à l'entriée du Petit-Belt); un en désembre dans la Baltique (Kieler Bucht); un en férrier (dans la Baltique, côtes de Holstein); un en avril (côtes de Landskrone).

Dans le courant de 188ב, M. W. Flower a fait connaitre unc ispèce nouvelle de ce genre sous le nom de IIyperondon planifrons, habitant les mers australiennes, et qui est parfaitement distincte de l'Hyperoodon rostratum. Le savant directeur du British Museum a publié le dessin de la tête, qui est déposéte dans ce riche Musée 1. C'est bien le représentant de notre Hypéroodon dans les mers australes.

C'est le seul genre de la famille des Ziphiö̈des dont unc espèce est confinée dans l'hémisphère boréal.

\section{PECHE.}

Itepuis peu, on a reconnu que les Hypéroodons, sans doute comme tous les Ziphiö̈des, fournissent du blane de baleine, et on leur fait aujourd'hui une chasse tris active sur les côtes est de Groenland, comme sur les côtes d'Islande.

Un baleinier de Peterhead, après avoir fait la pêche de la Baleine, s'est mis en 1877 à faire la chasse à l'Hypéroodon sur la côte nord-est d'lslande, et le succis semble avoir couronné complètement son entreprise.

En quelques années cette nouvelle pêche a pris d'énormes proportions; elle a eu un bon résultat, non seulement au point de vue industriel, mais également au point de vue do la zoologie; celui qui l'a entreprise a été ì mème de trancher une question qui tenait les zoologistes en suspens. L'Hypéroodon auquel on avait donné le nom spécifique de latifrons, à cause de lénorme développement de ses crêtes sus-maxillaires, formc-

${ }^{2}$ Flower, of the cranium of a new species of IIyprodon (jlanifrons) Proc. Zool. Soc, nıy 2, 1882. 
1. il une espèce distincte, ou, comme Eschricht l'avait dit, est-it le mâle de l'Ityperoodon rostratum?

Les nombreux individus de tout âge et de tout sexe pichis dans les mêmes parages et dont le capitaine Chieftain de Kerkealdy avait eu soin de distinguer les sexes, ont permis de: trancher la question dans le sens du savant cétologue de Copenhague.

En 1880, le capitaine David Gray ', commandant de l'Eclipse (Whaling steamer), harponna trente-deux individus et fit connaître les lieux où se tienuent principalement ees Citacés : c'est, d’après lui, à l'ouest et à l'est du Groenland. II cite particulièrement Iudson-Bay et le détroit de Davis jusqu'au 70" nord; autour et à l'est de Farewell, autour d'Islande, les côtes est du Groenland jusqu'au $77^{\circ}$ nord, à l'ouest de Spitzberg, à l'est de l'lle des Ours, à la latitude 72. et longitude $19^{\circ}$ est, Strait-Belle-Isle, à l'ouest et à l'est de la Nouvelle-Zemble.

En 1881, les baleiniers écossais seuls en prirent cent et onze, et en 1882 on a commencé la pêche aux Frobisher-Strait, oì le capitaine Chieftain de Kerkealdy en captura vingt-huit.

Pendant une seule campagne on en a pris jusqu'a quatre cent soixante-trois.

Le 27 avril on avait capturé le premier animal, et au mois de juin on fut obligé de jeter du charbon pour faire de la place à bord.

Un seul baleinier en a capturé, pendant les mois de mai ef de juin, deux cent et trois individus, dont nonante-six måles adultes, cinquante-six femelles et cinquante et un jeunes màles.

En 1883, onze navires ont pris cinq cent trente-cinq Hyperoodons, dont cent cinquante-sept par l'Éclipse seul.

En 1884, le principal navire n'en a plus capturé que cinquante-six.

La même année (1881), deux schooners de Vardö, Haalut Company, se livrent ì la píche de l'Hypéroodon, en se rendant

¿Davio Gra:. Pioc. Zoo'. Soc., 1885, p. 726. 
dans la direction de l'Islande et capturent à la fin de la saison neuf individus, produisant neuf ou dix tonnes de lard.

Cette pêche sera rapidement arrivée à son déclin.

\section{MUSÉES.}

Si, à l'époque où Cuvier a écrit ses Recherches sur les ossements fossiles, il n'existait pas un squelette d'Hypéroodon au Muséum de Paris, il n'en est plus de même anjourd'hui : on en trouve maintenant dans la plupart des musées.

En effet, nous en voyons à Amsterdam, à Belfast; à Berlin un squelette des Feroë; à Bruxelles, d'un animal capturé dans l'Escaut en 1810; à Caen, des individus mâle et femelle qui ont échoué sur la côte de Calvados; à Cambridge, au Musée de l'Lniversité; à Christiania, à Copenhague on en voit plusieurs squelettes des deux sexes, provenant des Ferö̈; à Édimbourg, à froningue, à Hambourg, à Hull, à Leide, à Lille, également des Feroë; à Liège des ossements des côtes de France (Hillion) ot un squelette d'un animal de l'Escaut; à Liverpool, à Londres, aul College royal des chirurgiens; au British Museum, la tête du mâle des Iles Orcades; à Louvain, à Lund, à Oxford; à Paris, le squelette de Solenelles de l'embouchure de l'Orne, et à Stuttgardt, un squelette de Shetland.

\section{DESSINS.}

Il y a peu de Cétacés qui ont été aussi souvent figurés, et il ny en a pas de plus facilement reconnaissables que celui qui nous occupe; et, ce qui est plus extraordinaire, c'est qu'un des plus anciens dessins, celui de Hunter, compte encore aujourd'hui parmi les meilleurs.

Thom. Pennant (1776) publie un dessin de l'animal, tout en le plaçant parmi les poissons, avec le Cachalot et les Dauphins.

Lacépède en donne le dessin, planche 13, figure 3. 
Il en existe une figure coloriée par Voigt, d'après un individu femelle pris dans la baie de kíel en décembre 1801 .

Fréd. Cuvier ' en a publié aussi une figure. Pl. XVII, fig. 1.

On en trouve également une dans kirauss ${ }^{2}$, pl. XLII, fig. 1.

L'Illustrated News, le 17 novembre 1860, a publié un dessin, d'après l'animal échoué sur la côte de Kent.

Comme nous venons de le dire, Inunter a publié un très ban dessin de l'animal. L'Hypéroodon a été figuré également par Dael, Pontoppidan, Wesmael, Vrolik, Otto Torell, Gray.

Wesmael représente l'animal en chair, mais ne figure pas les sillons sous la gorge.

Vrolik donne un fort bon dessin et reproduit les sillons.

Une des bonmes figures est celle de Gray dans Erebus and Terror, qui reproduit également fort bien les sillons.

Une autre bonne figure se troure dans l'expédition suédoise au Spitzberg en 1831 sous la direction de Otto Torell. Malmgren faisait partie de cette expédition.

M. Cope figure l'Hypéroodon qui a été capturé sur la côte de Rhode Island.

Il existe, avons-nous vu plus haut, de très grandes différences dans la tête, surtout eutre les màles et les femelles. Les meilleures figures du mâle, pour ne pas dire les seules, sont celles du capitaine David Gray, qui a observé lui-même les individus de différents sexes en chair.

Les diverses parties du squelette ont été dessinées avec plus ou moins de soin : en 1783 Pierre Camper recoit une tête d'un marchand de Saardam, qui était encore intéressé dans la pêche de la Baleine au Spitzberg, et la fait figurer dans son ouvrage posthume, publié par son fils avec des notes de Cuvier.

Pander et d'Alton ont figuré également une tête d'Hypéroodon.

1 Fréd. Cuvier, Histoire nalur. des Célacés, pl. XVII, fig. 1.

2 Fréd. knauss, Das Thierreich in Bildern, 1851, pl. Xlil, fig. 1. 
Gray représente la tète du mâle dans Erebus and Terror.

Eschricht a fait reproduire la tête du mâle, vue sous ses trois faces, et celle de la femelle; il a fait dessiner aussi le foetus et son squelette, de grandeur naturelle.

Vrolik reproduit, indépendamment de l'animal, le squelette et les principaux viscères festomac, trachée-artère, cœur, intestins et appareil sexuel femelle).

Les planches XVIII et XIX de notre Ostéographie représentent le squelette et les crânes des deux sexes.

\section{PARASITES.}

L'Ilypéroodon héberge généralement des parasites et des commensaux.

Il nourrit régulièrement, parait-il, l'Échinorhynchus turbinella dans les intestins 1.

lirabbe y a trouvé l'Ascaris simplex.

Blainville fait mention du Monostomum delphini.

Ilaaland a trouvé dans l'estomac, des Nématodes qui lui paraissent être des Strongles.

IIaalland a ouvert aussi les poumons pour voir s'ils ne contenaient pas des vers parasites. II n'a rien trouvé.

Parmi les commensaux se trouve le Cyamus Thompsoni Gosse, qui s'établit sur la tête et s'y propage avec une abondance extraordinaire. Nous avons reçu, de notre ami MI. Flower', la photographie d'un morceau de peau de la tête, sur laquelle les Cyames sont si serrés les uns contre les autres, que l'on dirait une peau de chagrin. Ils ont été recueillis sur une femelle, capturée en 1882, probablement dans la mer de Baftin.

Diesivg, Syst. helm., vol. II, p 54 . 
Ce Crustacé a été décrit par Gosse en $1800 \%$ ', et le professeur Lütken fait du Cyamus Thompsoni de Gosse, le Platycyamus Thompsoni 2 .

Cie Cétacé héberge également la Penellu irussiromis, Stp. ot Lutk.

On a trouvé encore eomme commensal le Conchoderma iotion. Cinierii, qui semble hanter indistinctement la eorue des navires ou le corps de certains Cétacés.

"Gosse, On Some new or lille known marine animals, Ann. Mag. Nat. hist, XV1, 18\%3, p. 50, t. III. fig. 2.

${ }^{2}$ Lüткеx, Bidr. lill kundskab... Cyamus. Copenhague, 1875. 



\section{ZIPHIUS CAVIROSTRIS.}

\section{LITTÉRATURE.}

Coceo, A. Su di un Delfino rinvenuto nello Strello di Messina (I)elphinus philippii). Macrolico, Gions. dec Gabix. di Messixa, 18 1.

Anast. Coceo, Ueber einen in der Meerenge von Messina gefundenen Delphin. Earchsos's Arciuv, 1846, p. 101.

Paul Gervais, Mémoire sur la famille des Cétacés ziphioüdes. Axyales des sciences yaturelles, 亏̄e séric, t. XIV, 1850.

Duvernoy, Mémoire sur les caractères ostéologiques des genres nouverux ou des espèces nouvelles de Célacés vivants ou fossiles. ANNALES DES sciences natunelles, 5e séric, t. XV, 1851.

r. Fischer, Sur un crâne de Ziphints trouvé à Arcachon (Gironde). Comptes rendus de l'Académie des sciences de Paris, 6 août 1866.

P.-J. Van Beneden, Mémoire sur une nouvelle espèce de Ziphius de la mer des Indes. MéMolnes counonyés et autres MéMolres publés par l'Académe royale de Belgique, coll. in- $8^{\circ}$, t. XVI.

urrmeister, liauna argentina. Anvales del Nuseo publico de Bienos-Arnes, in-4\%. Buenos-Ayres, 1868. Entrega quinta. 
nalm, Ilvaldju i Sveriges Museer är 1869, Kosig Srexska VetenSKAP-AKAD. HANDLINGar. Band 9, no 2. Stockholm, 1871.

w. Turner, On the occurcnce of Ziphivs cavirostris, in the Shethend Seas. Edinburgh, 1872. Traxsact. ז. Soc. Edinb., t. XXV, p. $75 \%$.

J. an. scott and ror. r. s. Earker, Nolice of memoir on a Whale of the genus Ziphius, recently obtained near Duncdin, Now-Zcaland. Proc. ZooL. Soc., 1887, p. 542 (april).

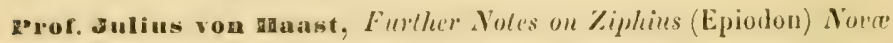
Zelandie. Proc. zooL. Soc, 1885, p. $\$ 910$. 


\section{HISTORIQUE.}

En 1804, on trouva sur le bord de la plage, entre le village de Fos et l'embouchure du Galegon, une tête plus ou moins complète, très pesante et très dure, complètement pétrifice en apparence. L'illustre auteur des Recherches sur les ossements fossiles la désigna sous le nom de Ziphius, terme employé par quelques auteurs du moyen âge. Le Ziphius curirostris devint le premicr et l'unique représentant de ce genre, ot Cuvier croyait l'espèce complètement éteinte.

Cette tète a été longtemps l'unique partie du squelette connute des naturalistes; Desmoulins "n reprit l'éturle in 1825, et, en la rapprochant des Hypéroodons, il montra qu'il avait apprécié les véritables aflinités de ce nouveau genre de Cétacés.

Paul Gervais trouva une seconde tête en 18300, également sur le bord de la Méditerranée, à Mresquiès; Hérault). Il parvint non sans peine a débrouiller l'histoire du Ziphius carirostris : il rapporte les têtes de Fos et d'Aresquiès à une seule ef mème espèce encore vivante, et démontre que ce mêne animal a été désigné déjà sous deux noms bien différents, dans les eaux de Nice. Risso l'a commu et désigné sous te nom de Delphi-

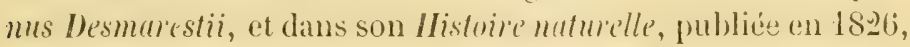
il figure une femelle venue à la côte. Cet animal n'est pas commun dans la Méditerrance, dit-il. - Nous en avons vu des restes dans son Musée à Nice. Sous le nom de Delphimus Philippii, le professeur Coceo a signalé lè mème animal dans le détroit de Messine vers 1840 .

Paul Gervais ne parvint pas sans peine ì faire triompher son opinion et à y rallier liallhésion des naturalistes. La tête décrite par Cuvier était toujour's considérée comme fossile. Durernoy, le successeur de Cuvier au Muséum, refusait d'admettre l'attribution de la tite de lós et de celle d'Aresquit's ¿t la mème espece animale. Pour justifier son opinion, Durernoy passa en revue les squelettes des Cétacés roisins, conservés dans 
Ie's galeries du Muséum, et il rangea le Ziphius d'Aresquiès parmi les Iypúroodons, sous le nom de IIyperoodon Gervaisii.

A l'époque où des doutes existaient encere sur l'identité des têtes de Fos et d'Aresquiès, nous avons comparé avee soin les deux têtes réunies dans les galeries du Muséum, et nous trouvons dans nos notes, qu'une partie du crâne d'Aresquiès a été scice en dessus ainsi qu'une partie de la face à gauche. L'os propre du nez ì droite manque. Le rostre est complet. Il est plus long que dans le Ziphitus des Indes ${ }^{4}$, et au bout il porte une espece de rostrule qui existe exactement de même dans l'individu de Fos.

Ce qui distingue particulièrement ce dernier, c'est que l'intermaxillaire, de droite surtout, est beaucoup plus excavé en dessous des fosses nasales, de manière qu’à la base du rostre, la partie supérieure de la face est profondément creuséc; cette excavation ne serait pas moins grande, si les parties qui manquent étaient remises en place.

Du reste, les intermaxillaires sont tous les deux complets dans cette région, ce qui était resté inaperçu, paraît-il.

Une autre différence, e'est que le cartilage du vomer est, comme dans l'Indicus, complètement ossifié dans le crâne de Fos, et peu dans l'autre.

Dans les trous sous-orbitaires il existe également une différence notable: le sous-orbitaire de gauche s'ourre au fond d'un profond sillon dans celui de Fos, tandis que dans l'autre il s'ouvre superficiellement, de manière qu'on en voit même deux à l'extéricur dans ce dernier. On ne distingue de ce côté qu'un seul trou dans celui de Cuvier.

La face indique ainsi de notables différences entre ces deux crânes, mais sont-elles sexuelles, individuclles ou spécifiques? Les modifications du cràne des Hypéroodons semblent faire croire qu'il existe des différences individuelles assez grandes dans la tête des divers Ziphioïdes, et nous ne croyons pas que ces différences dépassent les limites des variations spécifiques.

1 Nous crovions à cette époque que le Ziphius des Indes ilait une espèce distincte. 
Outre les exemples cités par Risso et Cocco, il y a également des Ziphius perdus sur les côtes de la Méditerrancée dont la date et le lieu d'échouement ne sont pas connus; la preuve en est que le Musée de Marseille posside la tète d'un animal sur l'origine duquel on ne trouve aucun renseignement.

Depuis lors, d'autres individus sont venus se perdre sur les borts de la Miditeranée, puis dans le golfe de Gascogne, et même dans la mer du Nord; de plus, nous savons aujourd'hui que le Ziphius curirustris, auquel appartient lit tète de Fos, est une espèce qui vagabonde, non seulement dans les mers d'Europe, mais également dans les eaux de nos antipodes et dans l'océan Pacifique.

On a été longtemps sans se douter que des Cétacés d'une méme espèce pussent habiter à la fois les deux hémisphères; nous en comnaissons cependant plusieurs exemples aujourd'hui, et nous pourons mème dire qu'aucun Ziphioïde, si ce n'est l'Hypéroodon, ne semble confiné, ni dans une mer intérieure, ni mème dans un océan.

Après 180̈0, le premier Ziphius qui échoue est un individu quise perd, en 1866 , sni la eôte de Villafinanca, dans un monent vì le professeur Hacedel y fait de's observations sur les animaux marins. Le sarant professenr de léna prépare le squelette, et, comme on le pense bien, cette belle pièce prend la route de Iéna, pour y enrichir les collections de l'Université.

En septembre 1878, les pêcheurs de Villafranea en capturent

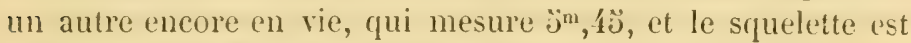
acquis pour le Musée de Florence.

En 1864, on décuurrit une tête de Cétacé ì Lanton, sur les bords de la baie d'Areachon, provenant également d'un Ziphius catirostris. C"est le premier exemple d'un animal de cette espèce trouré hors de la Méditerranée. Le $\mathrm{D}^{\mathrm{r}}$ Fischer en a donné la description et le savant aide naturaliste du Muséum fait connaitre en même temps les échouements connus dans la Méditerranée et à l'ouest de la France.

Le professeur Sir Turner publie, en 1872, un intéressanti TONE XLI. 
travail sur la présence du Ziphius cavirostris sur les côtes de Shetland, et il passe en revue les Ziphius connus des naturalistes; il fait mention de ceux des Bouches-du-Rhône et des autres qui ont été observés dans la Méditerranée et signale les individus observés à la côte ouest de France, au cap de BonneEspérance et sur les côtes de la République Argentine 1.

Le professeur d'Édimbourg réunit le Pétrorhynchus, le Ziphius indicus et l'Épiodon chatamensis dans une seule et même espèce.

Sous le titre: Zijhlioüde fossile 2 , le professeur Capellini a publié un mémoire accompagné de fort beaux dessins dans lequel il fait la description d'un crâne d'un Ziphiö̈le mis au jour, qui rappelle parfaitement le Ziphius cavirostre vivant encore dans les eaux de l'Italie.

Parmi les collections, que Castelnau avait recueillies au cap de Bonne-Espérance et aux Indes, se trouvait une belle tête de Cétacé que nous arons décrite sous le nom de Ziphius indicus. Elle nous paraissait d'abord bien différente des espèces connues, mais nous sommes persuadé aujourd'hui qu'elle appartient à une seule et même espèce qui est dispersée dans toutes les mers.

Depuis lors Burmeister dans sa Fauna argentina a fait mention de ce même Cétacé, qu'il a figuré d'après un animal qui a été capturé sur les côtes de la République Argentine au mois d'août 1883. Le savant Directeur du IIusée de Buenos-Ayres en donne une excellente description anatomique. Il croit à l'existence de plusieurs espèces dans ce genre, dont il apprécie parfaitement les affinités, et il donne le nom d'Épiodon australe à l'animal qu'il décrit.

Le professeur Cope a donné le nom de Ilyperoodon semijunctus à un Cétacé dont le squelette a été remis au Musée

\footnotetext{
- Trans. roy. Soc. Edinburgh, vol. XXVI, 1872.

2 Cipellixi, Del Zifioide fossile, Reale Academa dei Lincei, anno 1884-
} 1885. 
national des États-Unis d'Amérique; M. Fred. True vient d'en faire mention dans les Proc. de ce Musée. Après la description du squelette provenant d'un jeune animal, M. True dit que nous avons eu raison, $\mathbf{M}$. Flower et moi, de regarder cet animal comme un Ziphius, plutôt que comme un Hypéroodon. Il donne les mesures en millimètres des divers os de la tête.

Nous trouvons ensuite ce même Cétacé dans les eaux de nos antipodes, et il parait même qu'il y est beaucoup plus abondant que dans les mers d'Europe. Sous le nom de Epiodon. Novce-Zelandia, le $\mathrm{D}^{\mathrm{r}}$ Jules von Haast a fait connaître un Ziphioïde, qui n'est autre chose que le Ziphius cavirostris de notre hémisphère.

Dans l'lllustrated London News, de 1867, figure un Ziphius: du détroit de Bass, qui se rapporte parfaitement au même animal.

Le professeur Sir W. Turner a reconnu, parmi les ossements rapportés par le Challenger, des restes qui ont tous les caractères du Ziphioïde qui nous occupe 1.

On possède aujourd'hui à Londres une tète et un squelette de la Nouvelle-Zélande, qui se rapportent parfaitement au Ziphius d'Europe. M. le professeur Flower, après une étude des diverses têtes connues, n'hésite pas à se prononcer sur l'identité de ces Cétacés de la Nouvelle-Zélande et des côtes de la République Argentine avec les nûtres. Il ne trouve pas de différence, dit-il, ni avec le Ziphius indicus ni avec l'Epiodon australe de Burmeister 2.

On a établi aussi l'Epiodon patachonicum ou australe, qui n'est également que le Ziphius dont nous nous occupons; c'est l'avis de Mil. Flower et Paul Gervais, qui est partagé aussi par le professeur Sir W. Turner.

1 Turner, The Zoology of the Voyage of H.M. S. Challenger, 1880, p. 27.

${ }^{2}$ Remarks upon Dr vov IIAST's Communication on Ziphius NovaZelandia, Proc. ZnoL. Soc., June, 1876. 


\section{SYNONYMIE.}

Ziphius cavirostris.

Delphimus Desmarestii, Risso.

- Philippii, Cocco.

Hyperoodon daumetii, Gray.

- Gervaisii, Duvernoy.

Epiodon australe, Burmeister.

- Novo-Zelandia, v. Haast.

- chatamiensis, Hector.

- patachonicum, Burm.

Ziphius indicus, Van Beneden.

Petrorhynchus capensis, Gray.

IIyperoodon semi-junctus, Cope.

Zipliius Grebnitzkii, Stejneger.

\section{CARACTERES.}

La taille du Ziphius cavirostris est un peu inférieure ì cell. de l'Hypéroodon. Cet animal est surtout reconnaissable aux deux dents relativement assez grosses qui percent les gencives au bout du maxillaire inférieur.

Ces dents sont en mème nombre dans l'Hypéroodon; mais, Jans les Ziphius, elles sont beaucoup plus fortes, et en forme de fusean, tandis que, dans les Iypéroodons, elles sont à peine plus grosses qu'une plume d'oie et sans renflement sensible vers le milieu de leur longueur. Dans la mandibule des Ziphius il y a des alvéoles, parfaitement distinctes, qu'on ne voit pas aussi distinctement chez l'Hypéroodon.

Ses caractires extéricurs sont : la partie supérieure du corps est d'un noir bleuâtre, la partie inférieure blanche; cette partie 
supérieure est marquée de nombreuses taches de forme ovale, de deux à trois doigts de longueur, semblable à une peau de léopard, dit J. von Haast.

L'animal porte un sillon de chaque côté sous la gorge. Yous ne pouvons pas bien juger d'après le dessin de Burmeister si les deux sillons forment un $\mathrm{V}$ comme dans le Micropteron.

Ce sillon est indiqué dans la figure publiée dans les Archives d'Érichson, d'après l'animal capturé dans le détroit de Messine.

La femelle échouée à New-Brighton, en 1878, avait également le sillon sous-maxillaire.

Il existe des differences sexuelles comme dans les autres Ziphioïdes. On a remarqué que le Ziphius mâle de la NouvelleZélande a les dents du maxillaire inférieur plus fortes que la femelle.

\section{DESCRIPTION.}

Nous ferons remarquer que Gervais a constaté la présence de petites dents à la mâchoire supéricure du Ziphius cavirostris d'Aresquiès; il les a représentées de grandeur naturelle pl.XXI, fig. 3, de notre Ostéographie. Gervais en a vu encore à la mâchoire inférieure. Burmeister en a vu également en dessus et en dessous; von Haast a fait la mème observation sur des Mésoplodons d'âge et de sexe différents.

Cés dents sans alvéoles doivent être considérées comme des dents ancestrales.

Les vertèbres sont peu nombreuses comme dans tous les Ziphioïles; nous en comptons ici en tout quarante-neuf : sept cervicales, dix dorsales, onze lombaires et vingt et une caudales.

Les trois premières cervicales sont soudées entre elles.

Burmeister a décrit et figuré l'animal en chair, le squelette isolé et en place, la tête rue sous ses trois faces, le cœur, la 
surface interne de l'estomac et des intestins, la cavité de la bouche avec la langue, tout l'appareil respiratoire, y compris le larynx.

\section{MOEURS.}

Nous ne connaissons rien des mœurs de cet animal. Il est probable qu'il ne diffère pas, sous ce rapport, des autres Ziphioïdes. Tout ce que nous en savons, c'est qu'il est teutophage comme les autres animaux de cette division.

\section{DISTRIBUTION GÉOGRAPHIQUE.}

Nous allons passer en revue les localités où la présence du Ziphius cavirostris a été constatée.

La tête décrite par Cuvier, dans ses Recherches sur les ossements fossiles, a été découverte à Fos, sur le bord de la Méditerranée, en 1804.

Vers 1820 un individu a été capturé près de Nice.

En 1842 un individu a été vu flottant dans le détroit de Messine; un autre vers la même époque a été reconnu sur les côtes de la Corse.

En 1830 on a trouvé sur la plage d'Aresquiès (Hérault) une tête provenant d'un animal qui y a échoué au mois de mai 1.

Depuis lors d'autres individus ont été reconnus dans la Méditerranée; P. Gervais en cite des Bouches-du-Rhône, de Nice, de la Corse et du rlétroit de Messine.

Tout récemment, en septembre 1878, on a fait encore une capture à Beaulieu (Villefranche).

On connaît aujourd'hui les ossements de sept Ziphius différents, recueillis sur les bords de la Méditerranée.

2 Gerva:s, Instilut, 9 octobre 1850. 
On a constaté ensuite la présence de ce même Ziphius sur les côtes océaniques de France; en 1864, on a recueilli une tête à Lanton, sur les bords du bassin d'Arcachon. Cette tête a été remise à M. Fillaux. Le $D^{r}$ Fischer en fait mention dans les Comptes rendus de l'Académie des sciences de Paris 1.

Un mâle de cette même espèce est allé se perdre ensuite sur les côtes d'Irlande, et nous en connaissons qui ont pénétré dans la mer du Nord; il en est venu à la côte aux îles Shetland; le Musée d'Édimbourg en possède la tête, et le professeur Sir Turner l'a parfaitement reconnu; après l'avoir comparé avee les autres Ziphius, il fait remarquer que celui de Fos, découvert en 180', appartient bien à la même espèce, qui a été trouvée dans des mers si différentes.

La présence de ce Ziphius a été reconnue ensuite dans le Kattegat.

Sur la côte de Suède, non loin de Götenborg, sont venus échouer, à quelques années d'intervalle, deux Ziphius de la même espèce, dont l'un est conservé au Musée de Götenborg et l'autre au Musée de Stockholm.

Le premier de ces squelettes provient de l'animal échoué en 1867 ì Bahuslän (Suìde). Celui du Mlusée de Stockholm, qui est remarquable aussi par sa belle conservation, provient d'un animal perdu dans le voisinage de Marstrand (Suède), en 1872. Il a la huitième et la neuvième dorsale malades.

En dehors des mers d'Europe on a reconnu ce Ziphius à plusieurs reprises.

D'abord nous ne doutons pas que ce ne soit le même animal qui a été capturé par Castelnau, et que nous avons appelé Ziphius indicus 2 , ainsi que le jeune mâle de l'embouchure de la Plata qui a été décrit et figuré par Burmeister.

1 Soùt 1866, p. 271.

2 Ce riest pas dans la mer des Indes, comme nous l'avons cru, mais au cap de Bonne-Espérance que cette tète a elé recueillie; nous trouvons dans un altoum de Castelnau: L'Hyperoodon du Cap a été jeté à la cóte au fond de Table-Bay dans un ouragan en juillet 18:16: il avait 27 pieds de long, 
Gray et Owen ont fait connaitre un second Ziphius du ('ap) de Bonne-Espérance, que Gray a appelé Petrorhynchus pour rappeler la solidité du rostre. D’après l'âge, le cartilage susvomérien est plus ou moins complètement ossifié, et le Petrorliynchus n'est qu'un individu adulte.

L'océan Pacifique est également visité par ce Cétacé. Nous en avons vu deux têtes et trois mandibules au Musée de SaintPétersbourg, rapportées du nord du Pacifique par Wosnessearki. Ce dernier renseignement nous a été fourni par le docteur Brandt, directeur du Musée de Saint-Pétersbour'g.

Von Nordmann fait mention, dans sa Paléontologie de la Russie méridionale, d'une tête de Ziphlius de l'ile Saint-Paul, qui lui a été communiquée par le docteur Siemaschko.

Est-ce encore le même animal dont Iloseley a rapporté une grande partie du squelette des îles Falkland, sous le nom d'Épiodon? (Challenger).

Le Challenger a aussi rapporté plusieurs caisses tympaniques de Ziphius, retirées du milieu du Pacifique, au sud des îles Tahiti, à 2,33æ brasses de profondeur.

A propos d'un Ziphioïde du détroit de Magellan, Paul Gervais faisait l'observation suivante : "Pour dire toute ma pensée, je ne serais pas étonné qu’il en füt encore de méme pour les Zirnius winicus et Carexsis, c'est-à-dire, que ces derniers ne fussent également de la même espèce que le Ziphius de la Méditerranée. 》

Nous partageons complètement cet avis aujourd'hui.

Ce même Ziphius se rend ensuite jusqu'au nord du Pacificque: l'Institution Smithsonienne a envoyé un naturaliste, Stejneger, à l'ile de Behring, pour y chercher des restes de Stellère, et il a rapporté, avec plusieurs ossements intéressants de Cétacés, une tête de Ziphius cavirostris, parfaitement conservée. On lui a donné à tort un autre nom spécifique : Ziphius Grelnitskii, Stejneger. Avec ce même animal se trouvait encore un autre

etail gris, a donne :00 bouteilles d'hule très bonne et à laquelle les gens du pays allribuent beaucous de verlus médicinales. 
Ziphiölde que l'on ne connaissait qu'en Australie, le Berardius Bairdii.

Nous le trourons également dans les eaux de nos antipodes, et en plus grande abondance, parait-il, que dans nos eaux d'Europe.

En 1863, le professeur Y. Haast fait part à la Société Zoologique de Londres de la présence d'un Ziphius dans les eaux de la Nouvelle-Zélande 1.

A la séance du mois de juin $1876^{2}$, M. le professeur Flower exprime l'avis, confirmé depuis, que le Ziphins Nove-Zelandice ne diffère pas du Ziphius cavirostris et qu'il n'existe qu'une seule espèce vivante dans ce genre.

Deux individus ont échoué, l'un le 17 novembre 1878 à

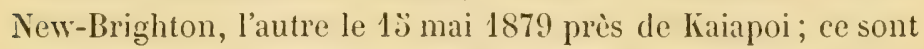
deux femelles : la première avait 19 pieds, la seconde 21.

En 1883, on a capturé encore un Ziphitus cavirostris sur les côtes de New-Jersey.

La dernière communication sur les Ziphius de la NouvelleZélande est celle de MII. John H. Scott et T. Jeffery Parker, de l'Université d'Otago (Nouvelle-Zélande). Ils informent M. Flower qu'une jeune femelle de Ziphius a été capturée vivante à Warrington, au nord de Dunedin (Nouvelle-Zélande), en novembre 18843 .

L'Epiodon chatamiensis, décrit dans les Trans. New-Zealand Institute, vol. V, est un mâle de cette espèce.

Le Ziphius cavirostris se rend également de l'autre côté de l'Atlantique : le professeur Cope a signalé sur les còtes de la Nouvelle-Angleterre une femelle de 19 pieds 4 pouces, qui a été capturée en 1883, à New-Jersey.

De manière que nous connaissons divers crânes et squelettes de la Méditerranée, un du golfe de Gascogne (1864), deux

I Z. S. L. 4 décembre $188 \overline{\text { J }}$

${ }^{2}$ Professor Flower, Remarlis upon Dr vos HaAst's communication on Ziphius Nova-Zelandia, Proc. Zool. Soc., June 6, 1876.

s Zool. Soc., London, 15 mars 1887. 
du Kattegat, deux du Cap, un du Brésil, un de la NouvelleZélande, un des mers de Chine et au moins deux du Pacifique, et tous ces crânes se rapportent fort bien à une seule espèce, ce qui nous permet de dire que le Ziphius cavirostris est un animal cosmopolite comme le Cachalot.

Indépendamment de ces divers Cétacés connus, nous pouvons encore ajouter que le Challenger a rapporté des mers du Sud une tête de ce même animal.

Nous avons vu également un rostre, provenant des Phosphate beds de la Caroline du Sud, au Musée du Collège royal des chirurgiens, à Londres, mêlé à des ossements d'animaux terrestres, parmi lesquels se trourait une dent de Megatherium (?) Ce rostre était perforé par des tarets. Nous avons vu un rostre, rongé de la même manière, qui était rapporté au Ziphius densirostris, et qui avait été recueilli sur la côte d'Afrique. On ('n a trouvé dans le sable d'Anvers plusieurs, rongés exactement de la même manière.

En somme, nous connaissons aujourd'hui des restes d'individus recueillis dans la Méditerranéc, à Fos (Bouches-duRhône, G. Cuvier); à Aresquiès (Hérault, Gervais); en Corse (Doumet); au détroit de Nessine, Villefranche (Haeckel); dans le golfe de Gascogne, à Arcachon (Fischer); aux îles Shetland (Turner); aux côtes de Suède, Kattegat (Ialm); dans l'hémisphère austral, au cap de Bonne-Espérance (Van Beneden, Gray et Owen); dans la République Argentine (Burmeister); sur les côtes de Patagonie, aux États-Unis d'Amériqque et à la Nouvelle-Zélande.

\section{MUSÉES.}

On conserve aujourd'hui des ossements de cette espèce dans plusieurs Musées.

Le Muséum de Paris en possède deux têtes: l'une décrite par Cuvier, l'autre par Paul Gervais. Toutes les deux proviennent, comme nous l'avons vu plus haut, d'individus capturés dans la Méditerranée. 
Le Musée de la ville de Marseille possède le squelette d'un animal qui a péri sans doute sur les côtes voisines, mais dont il n'est pas fait mention dans les livres qui traitent des Cétacés.

On conserve encore des têtes et des squelettes provenant de Ziphius échoués sur les côtes de cette même mer intérieure, à Florence, à Pise et à Cette.

Le squelette de Florence provient de l'animal capturé à Villefranche en septembre 1878 .

Ln squelette complet, préparé par le professeur Hackel en 1866, est conservé au Musée de l'Institut Zoologique de Iéna.

Au MIusée de la Société scientifique d'Arcachon, on voit une tête de cette même espèce qui a été recueillie, en 1864, à Lanton dans la baie d'Arcachon.

A Dublin on possède le squelette complet d'un animal échoué sur les côtes d'Irlande.

On en conserve deux têtes à Édimbourģ; une d'elles a été rapportée de Shetland par Couthey 1.

Nous conservons, au Musée de Louvain, la tête qui a été rapportée par Castelnau de son voyage aux Indes orientales, et que nous avons décrite sous le nom de Ziphius indicus.

A Götheborg, on conserve au Musée le squelette de l'animal qui a échoué, en 1867, à Bohuslän.

Le IIusée de Stockholm renferme le squelette complet de l'animal qui est venu à la côte, en 1872, dans le voisinage de Marstrand (Suède).

Nous avons vu deux têtes de cette espèce dans les galeries du Musée de Saint-Pétersbourg, toutes les deux de l'océan Pacifique.

A Charleston on conserve le squelette de l'animal qui a été décrit sous le nom de Hyperoodon semi-junctus, Cope?.

3 Turner, On Ziphius cavirostris, Transactions roy. Societr, Édimbourg, t. XXVI, p. 750 .

2 Proc. Acad. Sc. Philadelphie, 186 ö et 1869. 
Au IIusée de Washington on voit aujourd'hui une tête de cette espèce rapportée de l'île de Behring, par Stejneger.

On conserve également un squelette de cette espèce au Muscie de Buenos-Ayres. Le savant directeur Burmeister en a donné une description.

A Christchurch, Canterbury Muséum (Nouvelle-Zélande), on conserve le squelette de l'animal décrit par Jul. von Haast.

Nous avons ru au Muséum de Paris un rostre de Ziphiö̈de fossile des côtes de Patagonie.

On voit enfin au Musée du Collège royal des chirurgiens à Londres un squelette de Ziphius de la Nouvelle-Zélande.

\section{DESSINS.}

Risso a figuré ce Ziphius dans son Itist. nat. de l'Europe méridionale, vol. III, pl. II, fig. 3, sous le nom de Dauphin de Desmarest.

L'animal entier est également représenté par Anast. Coceo

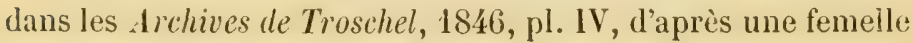
capturće dans le détroit de Messine.

On voit également un dessin de cet animal dans la Revue de Zoologie, 1842, pl. I.

Nous possédons aussi de bons dessins des Ziphius capturés sur les côtes de l'Amérique méridionale et de la NouvelleZélande; Burmeister' a représenté l'animal complet et a figuré les principaux viscères; James Hector a publié un bon dessin de la tête, de la mandibule et des dents.

Dans les Transact. de New-Zealand Institut, vol. V, Jul. von Haast a publié la figure d'une femelle de 19 pieds, échouée près de New-Brighton (Nouvelle-Zélande).

Dans l'Illustrated London News, vol. I, 1867, p. 97, figure un Ziphius observé dans le détroit de Bass.

Indépendamment de l'animal, les diverses parties du squelette ont été dessinées sur les lieux d'après des individus capturés dans les eaux de nos antipodes. 
La planche XXII, fig. t-11 de notre Osténgraphie, représente la colonne vertébrale, le sternum; planche XXI, les crànes des Aresquiès, de Buenos-Ayres, de Lanton (Gironde), de Fos (Bouches-du-Rhône), de Corse, du cap de Bonne-Espérance, de la mer des Indes.

Nous avons figuré le crâne du Ziphins capturé par Castelnau, la dent, la caisse tympanique en place et isolée, dans les Mémoires de l'Académie royale de Belgique, coll. in-8", t. XVI.

Gervais a figuré les dents du maxillaire supérieur dans sa Zool. et patéont. frans., planche XXXIX, et planche XXI de l'Ostéographie.

Jul. von ILaast a figuré le squelette et le crîne d'un animal de la Nouvelle-Zélande.

\section{PARASITES ET COMMENSAUX.}

On ne connaît jusqu'à présent qu'un Cestode et un ver rond qui peat être un Nématode ou un Echinorhynque; le premier est un Phyllobothrium agame, que le professeur Hieckel a trouvé dans l'épaisseur de la peau.

Le second est un ver très long, logé dans l'estomac d'un Ziphius échoué sur les côtes de Suède en avril 1867 ( $\mathrm{Ialm}$ ). Le professeur Sir Turner suppose que e'est un Echinorhynque; un nouvel examen est indispensable. Nous ne savons si cet animal a été conservé. 



\section{MICROPTERON SOWERBYI.}

\section{LITTÉRATURE.}

Sowerby, British Miscellany, t. I, 1806.

Rafinesque, Prćcis des découvertes sominlogiques. Palerme, 1814.

IIainville, Nouv. Bullet. des scicnces. Septembre, 1825, p. 159, (Bull. Soc. Philom., IV.)

EIsso, II ist. nat. Eur. mér., t. III, pl. 2, 1826.

Fred. Cuvier, De l'hisloire naturclle des Célacés. Paris, 1856.

Wu Mortier, Némoire sur le Delphinorhynque microplère. (Nouv. Més. Acad, noy. de Bruxelles, t. XIJ, 1859.)

YuxIey, Proc. Geol. Soc., 1846, p. 588.

Eschricht, Unters. über d. nordischen Wallhiere. Leipzig, 1849.

J. E. Gray, The Zoology of the Voy. of H.M.S. Erebus and Terror, p. 27, pl. V, fig. 2, 5 .

Paul Gervais, Zoologic et paléontologic francaises. Paris, 1839.

Tan Beneden, Sur un Dauphin nouveau et un Ziphlioide rare. Mémolres couronnés et autres Mémoires publiés par l'Académic royale de Belgique. Coll., in-8, t. XVI, 1865.

w. Andrews, On Ziphitus Sowerbiensis, captured in Brandon Bay, coast of Kerry, on the $9^{\text {th }}$ of 1 larch, 1864.

Eug. Deslongehamps, Observations sur quelques Dauphins appartenant à la section des Zipliiides. Bull. Soc. Lisw. de Normandie, 1866.

Agassix, Boston Sociely of nal. hist., novembre, 1867. 
Julius von marst, On a new Ziplioïl Whale. Proc. Zool. Soc. 1876, p. 7.

Elower, "A further contribution to the knowledge of the existing Ziphiöld Whales of the genus llesoplodon, containing a description of a Skeleton and Several Skulls of Cetaceous of that genus from the Seas of New-Zealand. "Proc. Zool. Soc., novembre 6, 1877, et Trans. Zool. Socicly, vol. X, 1878.

9. Ecinharit, Mesoplodon bidens en lilecext til den danslie I/avfanna. Copenhague, 1880.

Nalı, Ont Micropteron bidens, ơ Götcborys Nalurhist. Mus., III, Arsskr., 1831, p. 52 .

våm, Gölcborgs urelurlistoriska Muscum. Götcborg, 1882, p. 52.

Mrof. Turer, On a specimen of Mesoplodon bidens captured in Shelland. Jourix. of avit. And pHYs, t. XVI, april, 1832.

Dror. Therser, The index of the peleic brim... The analomy of a sicond specimen of Sowirby's Whale. Jounsal of Axatom. And P'Irsiol., octobre, $1855 \%$

ar Carl auvillius, The ostcology and exterior conformation of Sowerby's Whule (Hicropteron bidens Sow.), Stockholm, Académie des sciences, ociobre 14, 1883.

Fred : True, Descript. of a new Species of Dlesoplodon. Proc. OF UxiT. St. Nat. Museum, 1885.

- *ี. Iralm, Om Sowcrby's IIval. (Mesoplodon bidens) Stockholm. Ör. Vct.-Ak., $188 \%$ \%.

Dr Curl urivilius, Carl W. S. Osleolngic und äussere Erschcinung des Wals Sowerby's (Micropleron bidens). Stockholm, 1886, in.8*. Bihang k. Suensk, Vel.-dk., IIandl., 11 Band, no 10.

Thomas sonthwest and ofiltiam Eaghe Clarke, On lhe ocrurence of Sowerly's Whale. Axx. ANd HaG. N.t. IIIST, p. 5j, January, 1886. 


\section{HISTORIQUE.}

C'est au commencement du siècle que Sowerby, le célèbre ronchyliologiste anglais, fit connaitre, sous le nom de Physeter bidens, un curieux Cétacé mâle, échoué sur la côte d'Elginshire, en Écosse, près de Brodie-House. Sowerby l'a fait figurer dans ses British miscellamy.

Après une visite faite en Angleterre, Blainville décrivit ce même Cétacé sous le nom de Dauphin de Sowerby, Delphinus Sowerbiensis.

Lesson en a fait mention dans son histoire naturelle des Cétacés 1.

Un Cétacé, échoué sur les côtes de Sicile vers 1790, attira en 1814 l'attention de Rafinesque, qui le fit connaitre sous le nom d'Epiodon urganautus 2 . Il a plusieurs dents à la mâchoire supérieure, dit Rafinesque, aucune dent à l'inférieure, et pas de nageoire dorsale. Nous avons tout lieu de croire que c'est une femelle de cette espèce, dont la nageoire dorsale rudimentaire, ainsi que la dent du maxillaire inférieur, n’ont pas été apergues. Les dents de la màchoire supérieure dont Rafinesque parle, sont des dents rudimentaires caduques sans aucune constance, ni dans leur nombre ni dans leur volume.

Fréd. Cuvier fait mention de ce nouveau Cétacé dans la 33 e livraison de l'Histoire naturelle des Mammifères, et en 1829 son frère, G. Cuvier, le cita dans le règne animal sous le nom de Microptère proposé par A. Wagner.

En 182:, un Cétacé extraordinaire se perdit à l'embouchure de la Seine; c'était une femelle également. Blainville eut l'occasion de l'étudier et le fit comnaitre sous le nom de Delphinorhynchus Dalei. La peau en a été empaillée pour les galeries

3 OEuvres compl. de Ruffon. Paris 182x, 1. $12 \%$.

2 liafnespue. Précis des découvertes somiologiques. Palerme, 1814.

TOME XLI. 
de zoologie du Muséum de Paris, et la tête a été conservée dans les galeries d'anatomie comparée.

La même année, un autre individu vint échouer à la pointe de Sallenelles, embouchure de l'Orne, côte du Calvados; son squelette fut préparé pour le Musée de Caen.

Le 22 aout 1828, une femelle de 11 pieds se fit prendre vivante au Havre, et on la tint en vie pendant deux jours.

En 1849, Eschricht publia ses Entersuchungen über Nordische Talthiere et adopta également le nom de Vicropleron, proposé par A. Wagner.

Le nom générique a sourent été changé depuis, mais cest le plus ancien, celui proposé par $\Lambda$. Wagner et adopté par Cuvier et Eschricht, que nous devons conserver.

Le 31 août $183 \%$, une jeune femelle est renue à la côte prìs d'Ostende et a fait le sujet d'un mémoire de feu B. Dumortier. Le squelette a été heureusement conservé par Paret et se trouve aujourd'hui au Musée de Bruxelles.

En 1863, nous avons publié une notice sur ce squelette, dont nous avons figuré les principaux os.

Duvernoy, comparant la tête du Physeter bidens mâle, de Sowerby, qui est au Nusće d'Oxford, avec la tête du Muséum de Paris, provenant de la femelle du Havre décrite par Blainville, a cru deroir en faire deux espèces. (Mesodiodon Sowerlyyi et Micropteron). Il ne s'est pas douté que le mâle a une forte dent au milieu de la longueur de la mandibule et la femelle une très petite.

Depuis lors, un mâle a été capturé sur les côtes d'Irlande, dans la baie de Brandon, comté de Kerry, le 9 mars 1864. En 1869, M. Andrews a publié un mémoire sur cet animal qui est désigné sous le nom de Ziplinus Sonerbiensis. II. Andrews fait remarquer qu'un autre mâle a été capturé déjà sur les mêmes côtes d'Elginshire.

Ce mémoire est accompagné d'une planche fort intéressante reproduisant une photographie de la tête avec sa forte dent et le sillon en $\mathrm{V}$ sous la mâchoire inférieure.

Eug. Deslongehamps a publié quelques observations sur un 
Ityperoodon qui flottait à l'entrée de la mer de la Manche et dont la tête est conservée au Musée de Caen. Cette même notice renferme des remarques sur le Micropteron de Sowerby, dont le squelette presque complet est conservé au Musíe de Caen.

L'auteur ajoute igalement une note sur le Dioplodon Gervaisii 1.

En 1870, un autre individu de cette espèce a échoué à la même baie de Brandon, dont nous avons parlé plus haut, et le professeur Sir Turner l'a fait connaître dans les Transactions de la Société royale d'Edimbourg. Il fait aussi mention d'un squelette entier et adulte qui est conservé au Musée de la Société royale de Dublin. Le savant professeur d'Édimbourg résume dans ce mémoire tout ce qui est connu de cette espèce.

Dans une visite faite au Musíe de Christiania, nous avons trouvé une mandibule de femelle, sans aucune indication d'origine, mais qui a probablement été recueillie sur les côtes de Norwège. Nous en arons fait mention dans les Bulletins de l'Académie 2.

Lne capture faite en 1869 rend probable l'origine scandinavienne de la mandibule de Christiania. Au printemps de la susdite année, des pêcheurs suédois ont rencontré en mer un animal de cette espèce à Nordjän (Kattegat). Il flottait à la surface; ils l'ont remorqué et le squelette en a été heureusement conservé au Musée de Göthebourg. M. Malm en a fait mention dans sa notice sur les squelettes conservés dans les Musẻes de Suède.

Il est venu encore un Microptéron à la côte dans ces mêmes parages septentrionaux à l'est de Jutland, le 3 février 1880; c'était un jeune mâle.

Reinhardt a publié sur cet animal une notice, qu'il a accompagnée d'un tableau des captures connues avec l'indication des lieux et des sexes; il reconnait cinq mâles et quatre femelles

' Eug. Desloxgchamps. Observations sur quelques Dauphins zjplités, 1866. Bull. Soc. Lixn, de Normaxde, lome $X$.

2 ge sér., 1. XXII, 1866. 
qui ont péri sur les côtes de France, des îles Britanniques, de la Scandinavie et de Belgique.

Un exemplaire de cette espèce a fait son apparition en 1883 à Saltö près de Lysekil (Bohuslän), et a été l'objet d'une étude de la part de M. Carl Aurivillius. Il a été capturé vivant.

Nous avons trouvé, au Musée de Saint-Pétersbourg, un crâne fort incomplet, dont létat de conservation nous fait croire qu'il provient d'une ancienne collection; il n'y a aucune indication sur son origine.

Nous arons reconnu igalement le crâne d'une femelle au Husée d'anatomie d'Édimbourg.

Le Micropteron Sourerbyi n'est évidemment pas un animal des mers d'Europe seulement; il est cosmopolite comme la plupart des Ziphioïdes.

Sur les côtes des États-Unis d'Amérique, à l'île Nantucket, on a capturé un individu qui mesurait 16 pieds de longueur et dont le crâne est conservé au IIuséum de Zoologie comparée de Cambridge.

En 1867, Agassiz a montré la tête de cet animal au congrès de Boston. Il le regardait comme nouveau pour les côtes des États-Unis d'Amérique.

Une autre observation en faveur de son cosmopolitisme? a été fournie récemment par Léon Stejneger, qui a été à la mer de Behring; il en a rapporté une tête qui a été décrite sous le nom de MIésoplodon. M. Fréd. True en a communiqué une description et une figure dans les Proceedings du Musée national des États-Unis. Les légères différences signalées par II. True nous semblent des différences individuelles ou locales plutôt que spécifiques.

Capellini a décrit et figuré des ossements de Mésoplodon ou Microptéron qu'il serait sans doute difficile de séparer de l'espèce vivante 1 .

Le Hicroptéron qui nous occupe, est également représenté

s Capeluni. Resli fossili de Dieplodon e Mesoplodon. Bologna, 188 i. Mem. R. ACad. Da Bologia. 1885. 
dans les eaux de nos antipodes; nous en avons vu un squelette complet au Muséum d'histoire naturelle de Paris sous le nom de Oulodon Grayi, qui est si semblable à celui du Micropteron de nos mers que nous croyions avoir l'espèce ordinaire sous les yeux. Les affinités de l'Oulodon avec le Mesoplodon Sowerbyi sont remarquables, disait I'aul Gervais, en parlant du squelette provinant d'un mâle assez jeune qu'il venait de recevoir de M. von Haast, de Christchurch, Nouvelle-Zélande.

Le nom d'Oulodon a été proposé à cause des dents fort petites de la màchoire supérieure, qui se trouvent également dans les individus d'Europe. Depuis longtemps, Paul Gervais a reconnu ces petites dents non alveolées, indépendamment de la grande, dans la fenclle qui a échoué au Harre-de-Grâce.

Depuis cet envoi au Muséum, un second individu de la mìne espèce est allé échoucr à Little Bay entre Botany-Bay et Long-Bay, ì une petite distance de Sydney. Ce second individu a été signalé par II. líreft. Le professeur Flower pense que ce dernier est plutôt un Mesoplodon Layardi.

Sous le titre de A further contribution to the linowledge of the existing Ziphioid Whales, Gemus Mesoplodon, qui est synonyme de ISicropteron, M. W. Flower passe en revue les différentes espèces établies par les auteurs, et compare entre eux les nombreux et intéressants matériaux que les Musées de Londres ont reçus de leurs correspondants en Australie.

M. Flower fait remarquer que les Mésoplodons, échoués il y a quelques années sur nos côtes, étaient considérés comme les seuls survivints des nombreux Ziphiö̈des fossiles de nos sables d'Anvers; mais, si ces animaux sont devenus rares aujourd'hui dans nos mers d'Europe, des travaux récents nous ont appris qu'ils se trouvent en abondance dans la mer de nos antipodes; ces Cétacés sont représentés par différentes espèces, dont quelques-unes virent par gammes assez nombreuses, puisque, comme nous le verrons plus loin, on en a ru échouer jusqu'à vingt-cinq à la fois sur la même côte.

Dans l'Ostéographie des Cétacés, mon collaborateur P. Gervais, chargé de la rédaction des Ziphiö̈les, consacre un 
chapitre au Mésoplodon qui nous occupe et s'étend sur la partice historique, la synonymie, le squelette et le système dentaire. II consacre les pl. XXII et XXVI à la représentation du squelette et des dents.

A. H. Malm a publić depuis une notice sur cette espèce dans le Bulletin de l'Académie royale à Stockholm. Il dome la description des divers os des deux squelettes qui sont conservés au Iusée de Göteborg, et il accompagne sa notice d'une planche représentant le crâne, la mandibule, l'os hyoïde et les os du membre du .Mesoplodon, à côté de celui de l'Ilyperoodon.

Le dernier travail sur ce Cútacé est dù à la plume si exercée du professeur Sir Turner. Il a reçu, en 188.̆, par un de ses élèves, un mâtle adulte ch chair, capturé aux îles Shetland, et à l'aide duquel il a fait connaître plus en détail le squelette et les parties molles encore fort incomplètement étudiées. Il a fait part de ses observations à l'Association Britannique, à Aberdeen, le 11 septembre $1883^{\prime}$. Le travail a été inséré dans le Journal of anatomy and physiolog!y, October, 1883.

La dernière notice sur cet intéressant Cétacé est celle de Th. Southwell et de W. E. Clarke sur l'apparition d'un individu du sexe mâle, échoué vivant à marée basse sur les côtes du Yorkshire, à l'entrée de la rivière Ifumber. On a reconnu trop tard l'importance de eet échouement; on n'a recueilli que l'huile et on a abandomné le cadarre à la mer, croyant que c'était un Hyperoodon.

Cet échouement eut lieu le même jour que le professeur Sir Turner fit sa communication à l'Association Britannicque, à Aberdeen, sur le mâle capturé aux îles Shetland.

Au cap de Bonne Espérance, un Hicroptéron a reçu le nom de M. Layardi et le Dr v. Haast a donné le nom de M. Floweri à celui de la Nouvelle-Zélande. Nous avons comparé à Londres une belle photographie du $D^{r}$ v. Haast, avec la tête du Cap, et il ne nous a pas paru, ni à M. Flower, ni à moi, que ces Ziphoïdes appartinssent à une espèce distincte. L'habitat de ces

1 Report of The British Associalion, 1885, Aberdcen, page 1037. 
deux Ziphiöldes nest pas une raison de croire à une différence spécifique, dit M. Flower; au contraire : il n'y a pas de barrière pour les Cétacés entre la mer du Cap et celle de la Nouvelle-Zélande.

\section{SYNONYHIE.}

Physeler bidens, Suwerby, British Miscellany, t. I, 1806.

Delphinus edentulus, Schreber.

Delphinus Sowerbyi, Dauphin épiodon, Desmarets, mammif., p. 521, 1820.

Delphinorhynchus Dulei, Blainville, Bull. Soc. philom. septembre, $182 \%$.

Delphinorhynchus micropterus, Cuv. Règne animal, 1829.

Dauphin de Sowerby, Fr. Cuvier, de l'llist. nat. des Célucés, 1836, p. 218.

Inoplodon Sonerbiensis, Gervais. Zool. el Paléontol. Pranc. tab. 30, fig. 1, 1859.

Mesoplodon Sunerbiensis, Gervais, Ostéographie des Cétucés, p. 392, tab. 22 et 23,1880 .

Mesoploiton Sowerbiensis, Van Ben. Mém. Acad. Bruxalles, rol. X, t. III.

Ziphius Sowerbiensis, Gray, Catalog. of Seals and Whales, p. 350.

Ziphius Sowerbiensis, Gray, Supplément, p. 101.

Delphinorhynchus micropterus, Dumortier, Mém. Acarl., Bruxelles, 1839.

Diodon de Sowerby, Lesson, Cétacés, p. 127.

Aodon de Dale, Lesson, Cétacés, p. 153.

Mesodiodon Sowerby, Duvernoy, Ann. sciences natur. 183̈1, vol. XV, p. ๖ั.

Mesodiodon Micropteron, Duvernoy, Ann. sc. natur., p. 57.

Micropteron, Eschricht, Amm. May, nat. hist. 1852.

Ziphius bidens, Lilljeborg, Sieriges och norges Ryggradsiljur, 2 vol., in-8 ${ }^{\circ}$, Upsala, 1874. 
Micropteron bidens, Malm. Göteborgs naturhiska Museum, IIl Arsskrift, p. 32, 1881.

Iresoplodon bidens, Flower, A further contribution, 1878.

Gulodon Grayi, v. Haast. Proc. Zool. Soc., London, 1876, p. 7 .

Micropterus et Micropteron sont les plus anciens noms génériques et c'est définitivement le nom de Microptéron proposé d'abord par Wagner que nous adoptons.

Dans le complément des auvres complètes de Buffon, R. F. Lesson a fait mention de l'Aodon de Dale 1, et considère comme synonymes le Delphimus edentulus de Schreber et le Dauphin de Dale, de Blainville et de Fr. Cuvier.

Le Dauphin de Desmarel, que Risso a mentionné le premier, est désigné sous le nom de Diodon Desmarest, dans le complément; c'est le même animal.

Le Diodon de Sourerby du même auteur est également synonyme, de manière que la même espèce figure sous trois noms différents.

Desmarest cite également sous trois noms différents le même animal.

Fr. Cuvier parle du Dauphin de Sowerby, puis du Dauphin épiodon qui est la même espèce.

L'animal de la Nouvelle-Zélande auquel on a donné le nom de Gulodon Ilectori, comme celui du nord du Pacifique et que M. True a dédié à Stejneger, se rapportent l'un comme l'autre à une même espèce, celle qui nous occupe.

Le Mesoplodon Floweri, dont le Dr von IIaast a envoyé une photographie, n'est autre chose que le $\boldsymbol{M}$. Sowerbiensis.

M. Flower a eu à sa disposition le cràne du Mesoplodon Ilectori, de Cool'Strait, le squelette d'un adulte capturé dans Lyall Bay, le squelette du Vesoplodon Grayi, de la NouvelleZélande, le rostre et la mandibule d'un vieux mâle; et il trouve encore ces matériaux insuftisants pour se prononcer sur leurs caractères spécifiques.

Eschricit, Untersuch. p. כ̋0. 


\section{CARACTERES.}

A l'état adulte, la mandibule ne porte qu'une seule dent de chaque côté; elle est aplatie, logée dans une profonde alvéole vers le milieu de la longueur de l'os. Cette dent est beaucoup plus forte dans le mâle que dans la femelle.

La longueur du mâle, depuis le bout du museau jusqu'au milieu de la nageoire caudale, est de 15 à 16 pieds (anglais). La largeur de la queue a 3 pieds 10 pouces.

La nageoire pectorale mesure, depuis la tête de l'humérus, 1 pied 10 pouces et son plus grand diamètre est de $61 /$ p pouces.

On a vu des jeunes de 7 pieds de longueur accompagner leur mère.

Le Vicropteron Sowerbyi mâle a, sous la gorge, deux sillons qui s'unissent en avant et forment un V. M. Andrews représente très bien cette disposition dans la figure 2 qui accompagne son mémoire.

\section{DESCRIPTION.}

Comme dans tous les Ziphiö̈des, la nageoire pectorale est fort petite; elle ne dépasse pas la longueur de quatre vertèbres lombaires.

L'humérus n'a pas la grosseur d'une vertèbre lombaire et ne dépasse pas sa longueur.

L'avant-bras est long comme le bras et comme la main, y compris la région carpienne.

Le premier rang des os carpiens a trois os dont le médian est le plus volumineux. Le second rang en a également trois.

Le pouce ne compte qu'un seul os, un métacarpien; les métacarpiens des quatre autres doigts diffèrent peu entre eux.

L'index et le médian ont quatre phalanges, l'annulaire trois et le petit doigt, un.

Le premier squelette de cet animal a été décrit par Dumortier; il était mal monté par Paret. Plusieurs pièces n'étaient 
pas à leur place. C'était lo squelette de la femelle venue à la côte d'Ostende.

Dans la notice que nous avons publiée sur cette espèce, nous avons dessiné comparativement l'ouver'ure du canal des vertèbres dans les différentes régions du corps '.

Nous trouvons dans la colonne vertébrale : sept cervicales, neuf ou dix dorsales, dix ou onze lombarres et dix-neuf ou vingt caudales, en tout 46 .

Les deux premières cervicales sont soudées dans l'Oulodon du Muséum de Paris; dans le squelette d'une vieille femelle du Insée de Christchurch, les trois cervicales antérieures sont réunies.

J. Nurie a représenté l'estomac; nous avons fait dessiner la poche du larynx et ses rapports avec la trachée-artère. Le professeur Sir Turner a publić depuis lors un bon dessin de cette même poche, d'après un fuxtus de Bulchoptera Sibbaldii que l'on peut comparer avec celle-ci.

MII. Ray Lankester et Turner ont fait connaitre la structure de la dent qui est implantée dans une alvéole vers le milieu de la longueur de la mandibule; elle est remarquable chez le mâle par sa forme et son grand développement.

M. C. Aurivilliers a étudié avec soin le point de réunion des deux lobes de la nageoire caudale, caractère qui n'arait guère cttiré l'attention; généralement il y a une échancrure au milieu, tandis qu'au contraire ici c'est une saillie.

Nous arons eu l'occasion de voir quelques parties molles que Paret arait desséchées, et dont les principaux caractères étaient encore conservés. Ainsi les intestins montraient, à leur face interne, les mêmes alvéoles à peu près que l'on observe dans l'Hyperoodon, et qui s'étendent sans doute sur toute la surface interne de la muqueuse intestinale; c'est, croyons-nous, une disposition commune à toute cette famille.

La trachée-artère a le caractère général de la trachée-artère des Cétacís, c'est-à-dire, elle se bifurque, mais avant la bifur-

1 Loc. citat., 1. 41. 
cation elle fournit une bronche latérale, qui compte les mêmes cercles cartilagineux que les deux trones principaux.

Depuis le larynx jusqu’à l'origine de cette bronche supplémentaire, nous comptons dix-sept cercles. Plusieurs d'entre eux se divisent à leur tour et s'élargissent au point de prendre le double des autres.

Comme nous l'avons dit plus haut dans la partie historicue, à la section de biologie de l'Association Britamique à Aberdeen, le professeur Sir Turner a entretenu ses corfrères de l'organisation du Microptéron dont il a disséqué deux individus.

\section{MOEURS.}

Nous ne croyons pas que la visite de l'estomae ait fait connaître la pâture habituelle de ce Cétacé, mais nous avons tout lieu de penser que ce sont, comme dans les autres Ziphö̈des, les Céphalapodes qui forment leur nourriture ordinaire.

On possède un trop petit nombre d'individus pour savoir s'ils vivent par couples ou par schools. On ne peut rien conclure de la capture isolée de quelques animaux. Tous ceux que l'on a observés en Europe étaient isolés, tandis qu'on en a vu échouer plusieurs à la fois dans les eaux de nos antipodes.

M. Malm fils reproduit le tableau des individus connus échoués ou capturés et sur treize d'entre eux il y a six mảles, quatre femelles et deux dont les sexes sont inconnus; on peut supposer que ces animaux vivent par couples, puisqu'il y en a à peu près un nombre égal de l'un et de l'autre sexe.

La femelle qui s'est perdue sur les côtes d'Ostende était seule; elle a été surprise par la marée descendante. Les pêcheurs du port la trouvèrent échouée vivante et, d'après le rapport de Paret, qui a recueilli l'animal sur la plage, elle poussa des mugissements pendant les deux jours qu'elle a vécu; il parait que sa voix sourde et eaverneuse avait des rapports avec le beuglement de la vache. 
La femclle qui a péri le 9 septembre $182 \%$, ̀̀ un quart de lieue au-dessus du Havre, était dans le même cas; elle est venue à la côte en plein jour et vivait encore quand on l'a découverte.

Ces Cétacés sont plus connus en Australie, et les naturalistes de la Nouvelle-Zélande nous rapportent que l'on en a ru échouer sar les îles Chatam vingt-cing individus ensemble; une autre fois quatre ont échoué à la fois sur les côtes de la Nourelle-Zélande. Ceci ferait croire que ces animaux vivent plutôt par schools, au moins dans ces parages et ì une certaine époque de l'année.

\section{DISTRIBUTION GÉOGRAPHIQUE.}

Le plus anciennement connu est celui qui a échoué dans la Méditerranée en 1790, sur les côtes de Sicile, et dont Rafinesque a fait mention; nous n'en connaissons pas le sexe.

L.' second cas connu est celui d’un mâle qui a péri en 1800 ou 1804 sur les côtes d'Elginshire, en Irlande.

Nous connaissons ensuite une femelle qui est venue à la côte en 1823, au Havre-de-Grîce, et dont Blainville a donné la description.

La même année un mâle a péri sur les côtes du Calvados (Salenelles). Il n'est pas impossible qu'il formât un couple avee la femelle du Havre.

I)ix ans plus tard, le 21 août $183 \%$, nous avons vu périr une femelle à l'ouest du port d'Ostende; Dumortier en a fait la description.

En 1864, le 31 mai, un second mâte a péri sur les côtes d'Irlande (Brandon Bay, Coast of Kerry); c'est celui dont M. William Andrews a reproduit la tête d'après une photographie.

Plus au nord nous voyons ensuite périr un autre mâle, le 18 juin 1869, à Nordsjän, sur les côtes du Kattegat ou du Skager-Rak.

Cette même année 1869, une femelle périt sur les côtes des États-Unis d'Aınérique, la dernière à Rhode Island. 
Les côtes d'Irlande (Brandon Bay, Coast of Kerry), voient de nouveau périr un mâle en 1870 , le 31 mai.

Une autre capture est celle d'une femelle qui a péri le 3 février 1880 sur les còtes de Danemark, à Hevringholmstrand (Jutland).

Ln mâle a péri lannce suivante, en octobre 1881, sur les côtes de Suède, près de Marstrand.

Le 23 mai 1884, un mâle a été vu sur les côtes nord-est de Shetland (in Voxter Yoc). Un jeune de sept pieds l'accompagnait. Les marins l'ont attaqué et il est venu ensuite échouer sur la plage. C'est à peu près la même place où un individu a été capturé en avril 1881. Le professeur Sir Turner a reçu l'animal en chair et a fait connaitre le résultat de ses observations anatomiques à l'Association Britannique à Aberdeen, comme nous l'avons déjà dit dans la partie historique.

Le 6 août 188\%̈, un jeune mâle a été pris vivant sur les côtes de Saltö (Bohuslän).

Nous trouvons ce Microptère également hors des mers d'Europe.

Il visite l's côtes de l'Amérique septentrionale : d'après un crûne trouvé dans les parages de Nantucket, on estime que l'animal mesurait 26 pieds de longueur. Agassiz a fait part de cette découverte à l'Académie de Boston en 1866.

On trouve également ce Hicroptère au nord du Pacifique : l'Institution Smithsonienne a reçu de l'île de Behring une tête recueillie dans ces parages par Stejneger, qui avait été chargé de collectionner des objets d'histoire naturelle dans ces contrées.

Le professeur Sir Turner reconnaît treize captures ou échouements de cette espèce, sur le continent d'Europe ou des États-Unis d'Amérique, dont trois sur les côtes d'Écosse et deux sur les côtes d'Irlande.

Depuis lors on a vu encore un individu sur la côte d'Écosse et un sur les côtes d'Angleterre; MII. Southwell et Clarke ont signalé ces apparitions.

Le dernier échouement est celui d'un mâle adulte, qui a 
échoué vivant à marée basse à l'embouchure du II mber (Angleterre), le 11 septembre $188 \%$.

Le Micropteron Sowerbyi visite également la côte de Norwège; nous en avons trouvé une mandibule dans les galeries du Nusée de Christiania, que nous avons tout lieu de croire recueillie sur la côte du pays. Elle était sans indication d'origine.

Nous trouvons également ce Microptère dans la mer de nos antipodes. Il semble même beaucoup plus commun dans ces parages que dans notre hémisphère.

Il se trouve également sur les côles est de Patagonie ot aux îles Falkland.

M. Moseley en a rapporté le squelette d'un animal de 14 pieds, et il m’informe qu'il a reçu de Pandy-Point (détroit de Magellan) une dent de Mesoploton Layardi, en tout semblable à celles qui sont connues.

Comme l'Hyperoodon est représenté chez nos antipodes par une espèce distincte évidemment du même genre, le Microptéron est représenté de même dans l'autre hémisphère par une espèce qui a les dents extraordinairement développées, le II icropteron Layardi.

\section{MUUSÉES.}

Jusqu'à présent on ne connaît qu'un petit nombre d'individus capturés et dont le squelette ou le crûne sont conservés.

A Paris on voit la peau empaillée de l'animal ćchoué au Harre, en 182.

A Caen on possède le crâne et la colonne vertébrale de celui qui a péri sur les côtes du Calvados, à l'embouchure de l'Orne.

A Bruxelles se trouve le squelette de l'animal pris vivant près d'Ostende et préparé par Paret.

A Dublin (Royal Dublin Society), le squelette d'un animal qui est venu à la côte en Irlande.

Au Musée anatomique d'Oxford, on conserve la tête de 
l'animal qui a échoué sur les côtes d'Elginshire (Angleterre), et qui a été décrit par Sowerby, dims ses British Miscellany.

A Édimbourg on voit, au Nusée anatomique, un crâne dont l'origine n'est pas connue et le squelette d'un mâle adulte, capturé en 188 ' ou 1883 aux îles Shetland. Il figure au Musée anatomique.

A Güteborg se trourent deux squelettes de mâles du Kattegat (Nordjän).

$\mathrm{Au}$ Musée de Christiana se trouve la mandibule d'une femelle.

A Saint-Pétersbourg, au Musée de l'Académie, il existe une tîte mal conservée provenant de quelque ancisme collection, d'origine inconnue.

Au Musée du Collìge roval des chirurgiens à Londres, on voit un squelette et des crânes de la Nouvelle-Zélande. Un squelette de jeune mâle a été offert par M. von Haast au professeur Flower pour le Musée de Hunter.

Au Nusée de Stockholm se trouve le squelette du jeune mâle qui a été pris vivant en 1885 à Saltö.

Au Musée de zoologie comparée de Cambridge, on trouve le crâne, décrit par Agassiz, qui a été recueilli sur les còtes de Nantucket.

La tête de l'île de Behring est conservée au Musće de Washington.

A Christchurch (Nouvelle-Zélande) on conserve le squelette d'une vieille femelle qui a ses trois premières cervicales réunies.

Au sujet des ossements de cet animal, A. H. Malm cite les Musées d'Oxford, de Paris, de Caen, de Bruxelles, de Dublin, de Christiania, de Harvard, de Göteborg, de Dublin, d'Édimbourg et de Berlin.

\section{DESSINS.}

Il existe plusieurs dessins qui représentent parfaitement cet animal. 
Dumortier a figuré une femelle encore en vie, étendue sur la plage d'Ostende. Le dessin avait été fait d'après nature par un artiste qui habitait Ostende.

Andrews a reproduit une fort bonne photographie de la tète du mâle.

Nous trouvons un autre dessin de l'animal dans les Archives d'Erichson et dans l'histoire naturelle des Cétacés de Fréd. Cuvier.

C. Aurivillier's a publié un dessin d'un jeune mâle capturé vivant à Saltö.

On voit un beau dessin de la tête dans le British Miscellany, tome $1^{\text {er }}$, et dans la Bankisian Collection, au British Museum. Ce dessin est fait d’après la tête qui est conservée à Oxford.

Le squelette, le crâne et la mandibule avec les dents figurent dans mon mémoire qui a pour titre : Sur un Dauphin nouveru et un Ziphioüle rare!.

Duvernoy a publié un beau dessin de la tête du Micropteron Sowerbyi måle et femelle.

Dans son mémoire sur les caractères ostéologiques des genres nouveaux", nous voyons un dessin de la tête avec la mandibule, sous le nom de Mesodiodon micropteron, et un autre de la tête d'Oxford, sous le nom de Mesodiodon: Sowerbii.

Paul Gervais a fait dessiner la tête qui est déposée dans les galeries du Muséum sous le nom de Dioplodon Sowcrbiensis ${ }^{5}$; il a fait figurer aussi une partie de la mandibule avec les dents sous le nom de Mesoplodon Sowerbiensis.

Gray a publié un dessin de la tête du mâle avec mandibule et dent 4 .

Le professeur Turner a publié le dessin de la queue et l'anatomie du membre thoracique de l'individu capturé en $188 \%$.

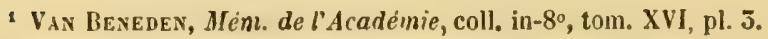

2 Ann. des scienc.naf., 5̄e sér., t. XV, 1851, pl. 2.

3 Zoologie et paleontologie françaises.

- Erebus and Terror. 
M. Malm fils a reproduit le dessin de la tête et de la mandibule du mâle.

Dans l'Ostéographie des Cétacés nous avons représenté la tête, las mandibules, les dents, la caisse auditive du mâle de Sallenelles (Calvados) pl. XXVI, fig. 1-4, le squelette de la femelle du Havre et de celle d'Ostende (pl. XXII, fig. 1-3.)

\section{PARASITES ET COMMENSAUX.}

Nous ne connaissons jusqu'ì présent qu'un seul parasite, et il est encore bien incomplètement étudié.

L'animal qui a échoué sur la plage de Sainte-Adresse en 1823 logeait sous la peau, dans l'épaisseur de la graisse, de nombreux kystes, dans lesquels se trouvaient des Trématodes ou piutôt des Cestodes, que l'on a pris pour des Monostomes, auxquels Blainville a donné le nom de IInostomum Delphini. Nous avons des raisons de croire que ce sont des scolex de quelque Phyllobothrium. 



\section{DIOPLODON EUROPAUS.}

\section{LITTERATURE.}

P. Gervais, Zool. el Palcont. franc., $1^{\text {re }}$ édit., 1850.

Eug. Deslongchamps, Obscrvat. sur quelques Dauphins. (Bulz. Soc. Lin.s. de Nordiandie, l. X, 1866.

Wischer, Nouv, archives du Musćum d'hist. nat., t. III, p. 68.

P. Gervals, Oslcographic des Cólacés. Paris, 1880, p. 40J, pl. X XIV. 



\section{HISTORIQUE.}

Le capitaine Vautier, au retour d'un voyage aux colonies, aperçut flottant sur l'eau, à l'entrée de la Manche, le cadavre d'un grand Cétacé; il fit enlever la tête, l'amarra avec soin à une corde et la fit porter ensuite à Caen, où elle est conservée au Musée.

Mon collaborateur P. Gervais a fait connaitre ce crâne en le désignant sous le nom de Dioplodon europæus.

Les avis des naturalistes sont partagés au sujet de la détermination de ce Ziphioïde, jusqu'à présent unique; aux yeux de quelques naturalistes, ce Cétacé représente un vieux mâle de Microptère ordinaire, dont la dent, au lieu de se développer vers le milieu de la longueur de la mandibule, se serait développée près de l'extrémité antérieure.

Tel est l'avis du $\mathrm{D}^{\mathrm{r}}$ Fischer et d'autres, qui pensent que cette pièce unique ne représente qu'une modification individuelle et qu'clle ne doit, par conséquent, pas figurer dans le relevé des espìces. Nous ne partageons pas cet avis; il n'est pas impossible que ce Ziphiölde soit propre à l'autre hémisphère et il se peut fort bien que jusqu'à présent il n’y ait eu qu'un seul individu capturé en Europe. N'avons-nous pas vu apparaitre une gamme de Pseudorques en 1861 que l'on n'a plus revue depuis et que l'on aperesut alors pour la première fois en Europe? Peu s'en est fallu qu'il n'y eût également qu'un seul individu de capturé!

\section{SYNONYMIE.}

Dioplodon curopceus, E. Deslongchamps.

Ziphius europaus.

Mesoplodon europaus.

Dioplodon Gervaisii. 


\section{CARACTÈRES.}

Ce Ziphioïde a la taille de l'espèce précédente et diffère surtout du Microptère par la dent ou la défense qui est placée près de l'extrémité antérieure de la mandibule.

On peut dire en faveur de l'identité de l'espèce avec le Micropteron Sowerbyi, que les dents des Ziphiö̈des semblent varier plus que celles d'autres Cétacés, et que l'on voit, dans une tête de Berardius, de chaque côté, une dent de moins qu'il ne devrait y en avoir. Dans l'Hyperoodon on voit également tantôt une dent de chaque côté, tantôt deux, quelquefois trois.

M. Flower admet six espèces dans le genre Micropteron : le Micropteron bidens ou Sowerbyi ; le M. europous; le M. densirostris; le $\boldsymbol{M}$. Layardi, du cap de Bonne-Espérance; le M. Hectori, de la Nouvelle-Zélande; et le M. Grayi ou l'Oulodon Grayi.

En parlant de cette tête en 1871 dans son mémoire sur les Ziphioïdes virants, M. Flower fait observer simplement que, jusqu'à présent, il n'y a qu'un exemplaire connu de cet animal.

\section{DESCRIPTION.}

Le rostre de la seule tête connue est solide et d'une forme un peu différente du rostre du M. Sowerbyi.

II n'y a qu'une seule paire de dents et elles sont placées à quelque distance de l'extrémité de la mandibule.

Ces dents montrent une partie radiculaire assez longue, dit Gervais, à peu près rectangulaire quoique un peu curviligne en arrière et faiblement oblique en avant, amincie au contraire dans le sens bilatéral. La couronne est triangulaire, très faiblement convexe en dehors, un peu échancrée en arrière et arquée en avant; elle est en partie couverte de cément et ce n'est que dans sa portion supérieure que l'ivoire est à nu. 


\section{MOEURS ET DISTRIBUTION GÉOGRAPHIQUE.}

Nous ne connaissons rien ni de leurs mœurs, ni de leur distribution géographique, puisqu'on ne connait que l'animal qui a été recueilli à l'état de cadavre à l'entrée de la Manche.

\section{MUSÉES.}

La pièce unique connue est déposée au Musée de Caen.

\section{DESSINS.}

Gervais a figuré les seules parties que l'on en connaisse : la tête et les dents.

\section{PARASITES.}

On a trouvé un Conchoderma (Otion) Cuvierii, attaché à la dent de gauche; nous l'avons vu encore en place.

La présence de ce commensal n'est-ce pas un indice que ce Cétacé est étranger à l'Europe ? 
SMITHSONIAN INSTITUTION LIBRARIES 